\title{
The forgotten ecosystem engineers: community and functional ecology of pioneer wood decay fungi in the canopy of beech trees
}

\author{
Rawlings, Anna
}

How to cite:

Rawlings, Anna (2018) The forgotten ecosystem engineers: community and functional ecology of pioneer wood decay fungi in the canopy of beech trees. Doctoral thesis, Swansea University.

http://cronfa.swan.ac.uk/Record/cronfa50898

Use policy:

This item is brought to you by Swansea University. Any person downloading material is agreeing to abide by the terms of the repository licence: copies of full text items may be used or reproduced in any format or medium, without prior permission for personal research or study, educational or non-commercial purposes only. The copyright for any work remains with the original author unless otherwise specified. The full-text must not be sold in any format or medium without the formal permission of the copyright holder. Permission for multiple reproductions should be obtained from the original author.

Authors are personally responsible for adhering to copyright and publisher restrictions when uploading content to the repository.

Please link to the metadata record in the Swansea University repository, Cronfa (link given in the citation reference above.)

http://www.swansea.ac.uk/library/researchsupport/ris-support/ 


\section{灌

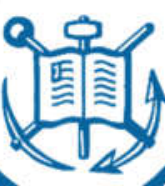 \\ Prifysgol Abertawe Swansea University}

The forgotten ecosystem engineers: community and functional ecology of pioneer wood decay fungi in the canopy of Beech trees

\section{Anna Rawlings}

Submitted to Swansea University in fulfilment of the requirements for the Degree of Doctor of Philosophy 


\section{Abstract}

Dead wood is a vital, diverse habitat which contributes substantially to woodland ecosystem function and biogeochemical processes. The major original source of woody debris is to be found in the canopy in the form of dead attached branches. Such branches also provide major habitat for the pioneer wood decay community which is known to influence the assembly trajectory, and therefore functioning, of later successional stages through priority effects. Very little is known however about the pioneer community's own structure and functioning. This thesis sets out to shine a light on these forgotten engineers of our woodland ecosystems.

Natural pioneer communities in dead attached beech branches were examined through isolation techniques and Sanger sequencing. Community structure was found to be driven predominantly by physical characteristics of tree branches, including their diameter and the gradient of decomposition. Pure spatial factors played a lesser role, indicating that mycelial spatial configuration results predominantly from the influence of environmental gradients. No relationship was found between community diversity and branch volume indicating that community establishment is driven by a race for space in a highly homogeneous resource.

Natural community function was examined using quantitative enzyme assays and untargeted GC-MS metabolomics. Laccase activity increase over the decay gradient suggesting development of an increasingly toxic environment. Untargeted metabolomics was applied to naturally decaying wood samples for the first time, highlighting a linear covariance of laccase activity with the metabolome. GCMS metabolomics was also applied in combination with direct shotgun LC-MS/MS proteomics to experimental wood systems. There was considerable evidence that a variable temperature regime may enhance lignin degradation in Oudemansiella mucida compared with stable temperature. A clear priority effect was exerted on the proteome of Exidia glandulosa by earlier arriving species, but not by different temperature regimes, suggesting that biotic interactions may play a more important role in the pioneer community than previously thought. 


\section{Declaration}

This work has not previously been accepted in substance for any degree and is not being concurrently submitted in candidature for any degree.

Signed

(candidate)

Date

\section{STATEMENT 1}

This thesis is the result of my own investigations, except where otherwise stated. Where correction services have been used, the extent and nature of the correction is clearly marked in a footnote(s).

Other sources are acknowledged by footnotes giving explicit references. A bibliography is appended.

Signed (candidate)

Date

\section{STATEMENT 2}

I hereby give consent for my thesis, if accepted, to be available for photocopying and for inter-library loan, and for the title and summary to be made available to outside organisations.

Signed (candidate)

Date 


\section{Acknowledgements}

I would like to thank my supervisors, Professor Dan Eastwood and Mike Fowler, for all their time and advice. To my mentor and friend, Dr. Suzy Moody, I extend a life-time of gratitude for her tireless support, advice and help, her words of wisdom and motivation and her endless provision of tissues. Special thanks also go to Professor Lynne Boddy for inviting me to find my way in this project in her laboratories at Cardiff University, for guiding me in developing such a huge passion for the pioneers of the wood decay world and for her infectious enthusiasm and sense of humour that never fail to light up the room. In Suzy and Lynne I have found two truly inspiring women of science and role models for the kind of scientist I will strive to be.

I could not have completed this work without the friendship of Eoin O'Connor through whom I was introduced to Dr. David Fitzparick and Professor Sean Doyle. Thank you, each of you, for taking such an interest in my work and for your generous donations of time and expertise and for funding LC-MS/MS analyses. I also could not have completed this work without the time, support and kindness dedicated to me by the busiest scientist I know: Dr. Ed Dudley, thank you.

I have made many friends during my studentship at Swansea University and special thanks go to James Taylor, Ben Clunie, Alex Dearden, Stuart, the two Toms and Chloe Robinson for making long lab days pass quickly with friendship, mirth and merriment.

To my family and friends who have never wavered in their belief I could finish this journey, especially Alice Bewick (for calling me Dr. Anna since day one), Stephen Thomas, Cloud Younger, Jack James, Branwen and Zeebee, Rhinanon Bevan, Christine Burrell and Ruth Philipson. I love you all and I look forward to spending a lot more time together from now on.

This thesis is dedicated to Tristan Silver, who was life's bright promise. 


\section{Statement of contributions}

The contributions of colleagues and students to the work presented in this thesis are explicitly detailed below:

Chapter 3: Damien Le'Goff and Rebecca Owens assisted with field work, DNA extraction and PCR procedures

Chapter 4: Dr. Ed Dudley ran GC-MS analysis

Chapter 5: Dr. Suzy Moody collaborated on experimental set-up procedures. Eoin O'Connor, Maynooth University, undertook protein extraction, peptide preparation and ran LC-MS/MS and protein sequence identification analyses. Dr. Ed Dudley ran GC-MS analysis.

Chapter 6: Dr. Suzy Moody collaborated on experimental set-up procedures. Eoin O'Connor, Maynooth University, undertook protein extraction, peptide preparation and ran LC-MS/MS and protein sequence identification analyses. Dr. Ed Dudley ran GC-MS analysis. 


\section{Contents}
Abstract . $\mathbf{i}$
Declaration .. $\mathrm{iv}$
Acknowledgements ...........................................................................................................................vi
Statement of contributions............................................................................................................. vii

Chapter 1 - General introduction.................................................................. 1

1.1 Importance of dead wood and dead attached branches in temperate woodland ecosystems

1.2 The assembly and structure of ligninolytic fungal communities in dead attached branches 2

1.3 The challenge of measuring fungal abundance in attached dead branches ............. 5

1.4 Fungal secondary metabolism and enzymatic decomposition of dead wood .......... 7

1.5 Statement of thesis aims and research objectives............................................................... 9

Chapter 2 - Materials and methods ............................................................... 11

2.1 Sampling methods employed in attached branches to characterise fungal communities .11

2.1.1 Study site

2.1.2 Selection of dead attached branches and generation of sampling locations along branch length

2.1.3 Calculation of branch volume and lateral surface area

2.1.4 Extraction of branch wood cores for analyses of enzyme activity and metabolome profiles

2.1.5 Dissection of branches into slices for community structure mapping. 13

2.1.6 Random stratified sampling, wood chip isolations and culture technique for identification of fungi resident in branches

2.1.7 Calculation of wood density and dry mass loss for branch slices and whole branches.. 16 
2.2.1 Preparation of pure cultures for DNA extraction . 16

2.2.2 Extraction, amplification and sequencing of genomic DNA from pure cultures. 16

2.2.3 Extraction of sawdust for fungal genomic DNA analysis. 17

2.2.4 Extraction of total gDNA from sawdust samples 17

2.2.5 Amplification of gDNA extracted from sawdust samples 18

2.2.6 Resolving the inhibition of PCR amplification of gDNA extracted from sawdust samples18

2.2.7 Bioinformatics of sequences of fungal gDNA 19

2.2.8 Measures and data analysis of fungal species richness and diversity within branches.. 19

2.2.9 Data analysis of fungal community structure within branches 20

2.2.9.1 Community structure data pre-treatment. 20

2.2.9.2 Unconstrained ordination of attached branch communities visualised by comparison with decay stage 21

2.2.9.3 Variation partitioning of environmental and spatial effects on attached branch community structure 22

2.2.9.4 Distance-based linear modelling of effects of predictor variables on attached branch community structure

2.3 Measuring fungal enzyme activity levels in attached branches 23

2.3.1 Extraction of total protein from branch wood cores 23

2.3.2 Bradford's assay and standardisation of protein concentrations across samples 24

2.3.3 Assays of activity level of hydrolytic enzymes 24

2.3.4 Assays of activity levels of oxidative enzymes. 25

2.3.5 Data analysis of enzyme activity levels of fungi in attached branch communities 26

2.3.5.1 Detecting differences in enzyme activity levels between competing and non-competing fungi26

2.3.5.2 Detecting changes in fungal enzyme activity levels over gradients of branch and community dynamics...

2.4 Profiling the metabolome of fungi in attached branches .......................................... 27

2.4.1 Extraction of fungal metabolites from branch wood cores 27

2.4.2 GC-MS analysis of metabolome profiles from branch wood cores 27

2.4.3 Deconvolution, pre-processing and alignment of GC-MS chromatograms 28

2.4.4 Statistical analysis of profiles of fungal metabolites from branch wood cores...... 29

2.4.4.1 Processing and normalisation of attached branch community metabolome. 29

2.4.4.2 Detecting differences in fungal metabolomes between competing and non-competing fungi29 
2.4.4.3 Detecting changes in the concentration of selected metabolites over the decomposition gradient

2.4.4.4 Distance-based linear modelling of effects of branch physical properties and fungal enzyme activity levels on the fungal metabolome in branch wood cores

2.5 Microcosm woodblock experiments........................................................................... 30

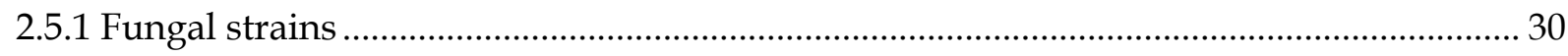

2.5.2 Set-up, experimental design and interaction assembly of microcosm experiments ........ 31

2.5.3 Determination of completion of replacement interactions .................................................. 32

2.6 Profiling the metabolome of fungi in beech woodblocks ......................................... 32

2.6.1 Extraction of fungal metabolites from beech woodblocks ............................................... 32

2.6.2 GC-MS analysis of metabolome profiles from woodblock samples................................... 32

2.6.3 Deconvolution, pre-processing and alignment of GC-MS chromatograms ...................... 32

2.6.4 Statistical analysis of profiles of fungal metabolites from branch wood cores ................. 34

2.6.4.1 Processing and normalisation of fungal metabolomes from woodblocks ............................. 34

2.6.4.2 Differential metabolomics of fungal metabolomes following stable and variable temperature treatment

2.6.4.3 Differential metabolomics of Exidia glandulosa metabolome following replacement interactions

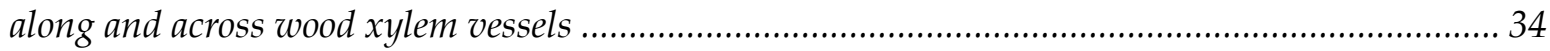

2.7 Profiling the proteome of fungi in beech woodblocks................................................ 35

2.7.1 Extraction of fungal metabolites from beech woodblocks................................................. 35

2.7.2 Direct shotgun LC-MS/MS analysis of peptide preparations from beech woodblocks.. 36

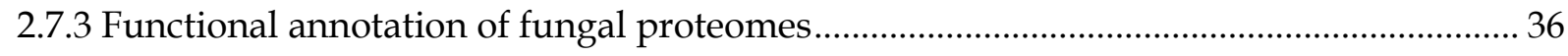

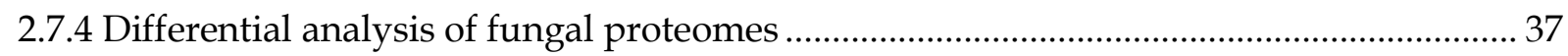

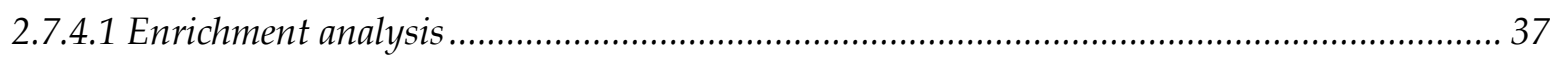

2.7.4.2 Discrimination analysis and T-tests applied to proteomes of fungi under different temperature

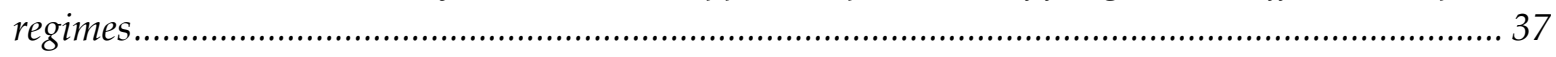

\section{Chapter 3 - The role of environmental and spatial processes in structuring} pioneer ligninolytic communities in beech branches.................................... 38

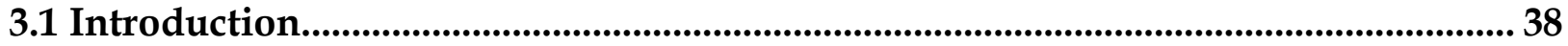

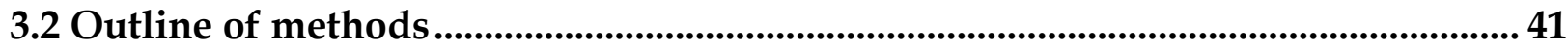

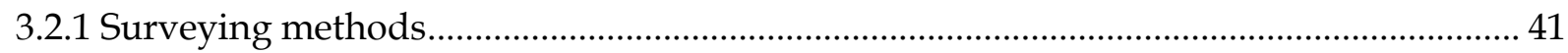

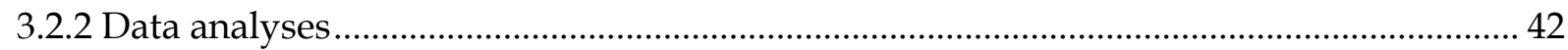

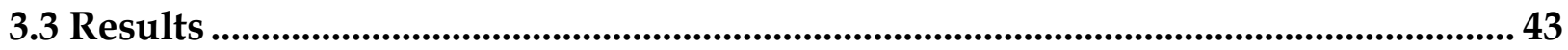


3.2.3 Quantification of the unique effect of environmental factors on community composition through variation partitioning 46

3.2.4 Effect of branch physical characteristics and spatial variables on community structure 48

3.3 Discussion 51

\section{Chapter 4 - Differential effects of physical branch characteristics and} community structure on enzyme activities and the metabolome of ligninolytic fungi in tree branches. 56

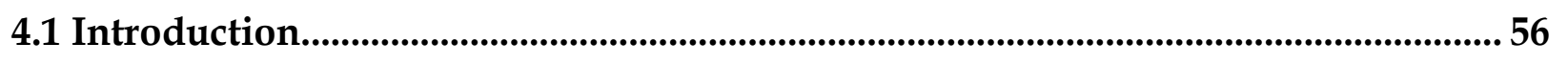

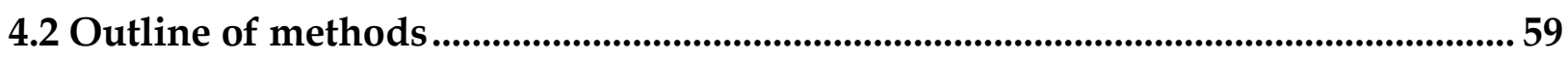

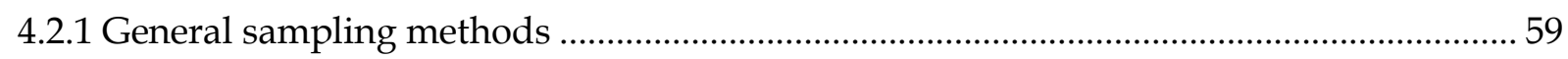

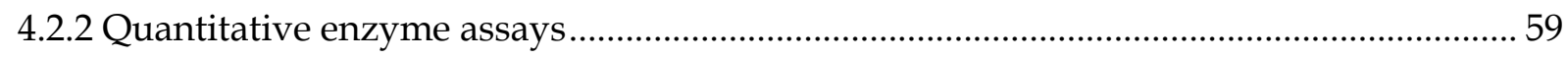

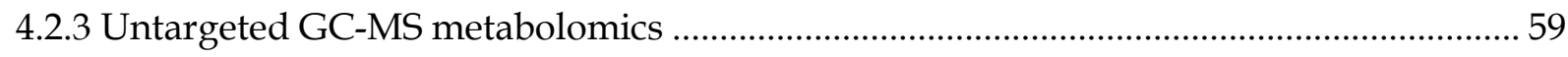

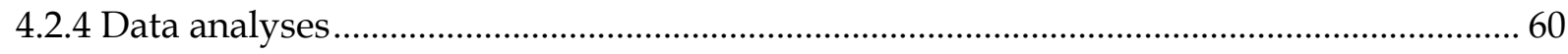

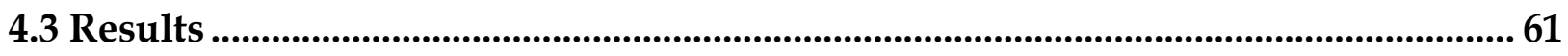

4.3.1 Sampling attached branch communities for enzyme activity and metabolomic profiling61

4.3.2 Oxidative and hydrolytic enzyme activity levels of competing and non-competing fungi62

4.3.3 Correlations of oxidative and hydrolytic enzyme activity levels with branch quality factors 63

4.3.4 Untargeted GC-MS metabolomics of attached branch communities.................................. 63

4.3.5 Differential metabolomics of attached branch communities.....

4.3.6 Distance based linear modelling of attached branch metabolomes, microhabitat factors and

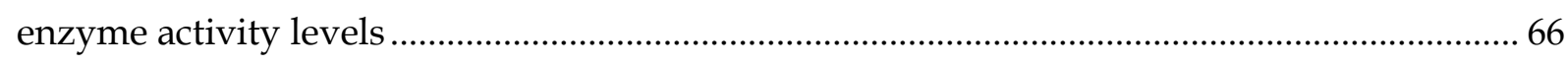

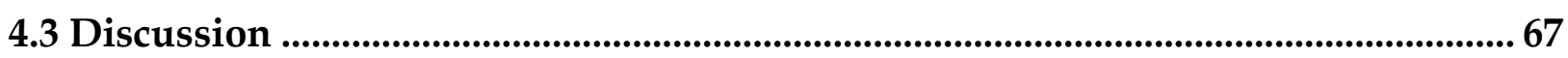

Chapter 5 - Untargeted metabolomics and proteomics reveal lasting effects of early-arriving species and wood anatomy on the metabolism of Exidia glandulosa 


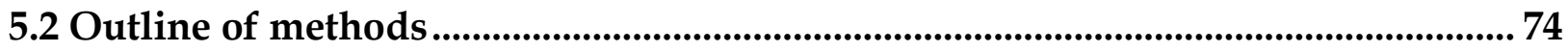

5.2.1 Experimental design and microcosm conditions .......................................................... 74

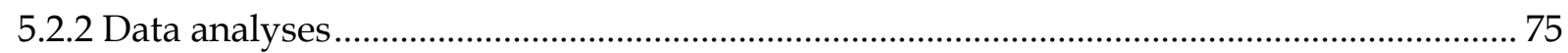

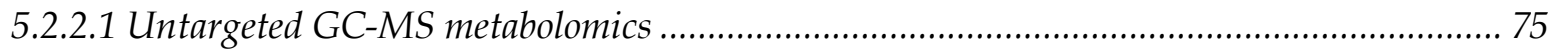

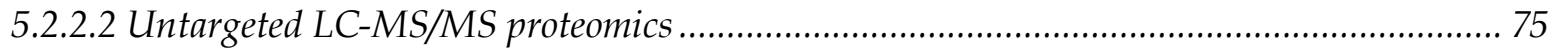

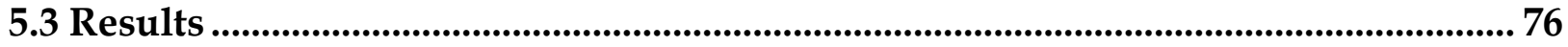

5.3.1 Untargeted GC-MS metabolomics of primary and secondary resource capture by Exidia

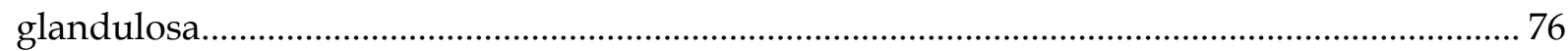

5.3.2 Effect of primary and secondary resource capture on the metabolome of Exidia glandulosa 76

5.3.3 Effect of early-arriving species on the metabolome of Exidia glandulosa following replacement along the wood vessels 78

5.3.4 Effect of early-arriving species on the metabolome of Exidia glandulosa following replacement across the wood vessels

5.3.5 Interactive effects of early-arriving species and wood anatomy on the metabolome of Exidia glandulosa.

5.3.6 Pathway enrichment analysis of Exidia glandulosa metabolome following primary and secondary resource capture. 84

5.3.5 Functional direct shotgun LC-MS/MS proteomics of the proteome of Exidia glandulosa following primary and secondary resource capture. .86

5.3.6 Differential protein expression of Exidia glandulosa following secondary resource capture

\section{Chapter 6 - Metabolic responses of two pioneer ligninolytic fungi to variable temperature during fungal-wood interactions ........................................................ 95}

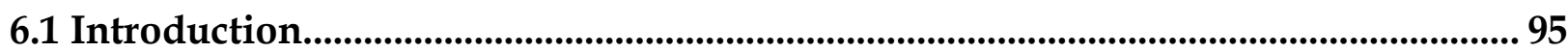

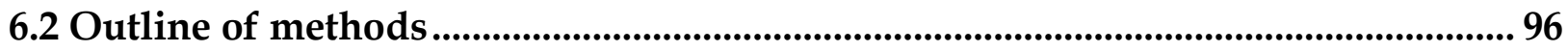

6.2.1 Experimental design and microcosm conditions ............................................................. 96

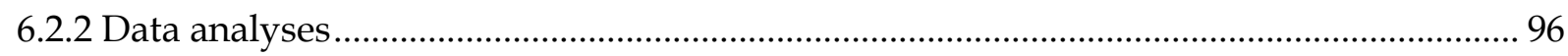

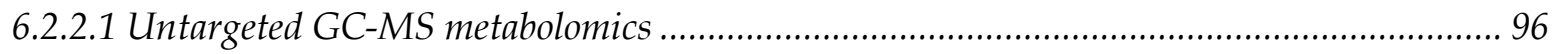

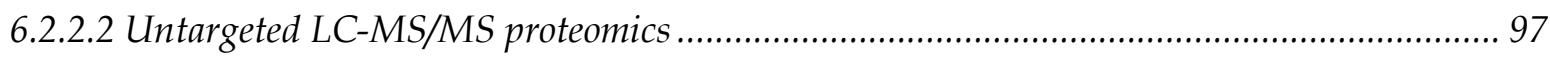


6.3.1 GC-MS metabolomics of Oudemansiella mucida and Exidia glandulosa under stable and variable temperature 98

6.3.2 Effect of variable temperature on the metabolomes of Oudemansiella mucida and Exidia glandulosa 98

6.3.4 Functional annotation of Oudemansiella mucida and Exidia glandulosa proteomes analysed by direct shotgun LC-MS/MS

6.3.5 Differential expression analysis of Exidia glandulosa and Oudemansiella mucida proteomes under variable and stable temperature 103

6.4 Discussion 106

Chapter 7 - Synthesis and future directions 109

7.1 Synthesis 109

7.1.1 Hypothesis 1: Pioneer ligninolytic community structure is shaped mainly by purely spatial effects rather than deterministic environmental processes 109

7.1.2 Hypothesis 2: The species richness of naturally formed pioneer communities increases with wood volume

7.1.3 Hypothesis 3: Metabolic processes in the primary decay community are linked to biotic interactions between species, to variable abiotic environment and with the gradient of decomposition

7.2 Strengths and limitations of experimental designs

7.3 Future research directions

7.4 Conclusions

References

Appendix I

Appendix II. 


\section{List of tables}

Table 2.1 Summary of branch dissection regime for mapping the structure of attached branch communities

Table 2.2 Primers used for amplification of phylum-specific fungal ribosomal RNA genes.

Table 2.3 Protein concentrations calculated per total protein extraction from branch wood core samples by Bradford's assay.

Table 2.4 Preparation of substrate solutions for quantitative fluorogenic assays of hydrolytic enzymes.

Table 3.1Physical characteristics and geographic locations of surveyed F. sylvatica attached branch communities

Table 3.2 Descriptive statistics for fungal colonies identified as belonging to the phyla Ascomycota and Basidiomycota.

Table 3.3 Variation partitioning by distance-based linear modelling highlighting the effect of the spatial and environmental partitioned predictor variables on variance in community structure. 48

Table 3.4 Distance-based linear modelling highlighting the effect of individual branch physical characteristics and spatial variables on variation in community structure. 49

Table 4.1 Properties of wood core samples and, their adjacent branch slices...... 62

Table 4.2 Wilcoxon signed rank test (competition) and Spearman rank correlations of enzyme activity levels and branch community variables.... .65

Table 5.1 T-tests of differences in compounds expressed by Exidia glandulosa following primary and secondary resource capture. 77

Table 5.2 Pathway Enrichment Analysis of Exidia glandulosa metabolome undertaken by Metabolomics Pathway Analysis (MetPA). 86

Table 5.3 Fisher's exact tests undertaken on pairwise treatment contrasts of Exidula glandulosa proteome following primary and secondary resource capture.

Table 6.1 T-tests of compound concentrations in the metabolome of Oudemansiella mucida under variable temperature compared with stable temperature 100

Table 6.2 T-tests of compound concentrations in the metabolome of Exidia glandulosa under variable temperature compared with stable temperature 


\section{List of figures}

Figure 2.1 Location of attached dead branches at Clyne Valley Woodlands study site in Swansea, South Wales, UK

Figure 2.2 Sampling methods employed in attached branches to characterise fungal communities.12 Figure 2.3 Strategy employed to determine orientation of extraction of wood cores for enzyme assays and metabolome profiling.

Figure 2.4 Applying random stratified sampling to wood slices. 14

Figure $2.5 \mathrm{~A}$ two-dimensional representation of the observed variation in a community structure data cloud and the application of permutation of significant P-values of the predictor variables in a distLM model 22

Figure 3.1 Unconstrained ordination of whole branch communities exhibiting low, medium and high levels of decomposition 44

Figure 3.2 Relative abundance of selected species over the decomposition gradient. 47 Figure 3.3 Relative abundance of selected species at order level over the decomposition gradient.47 Figure 4.1 PLS-DA of the metabolome of fungal residents in branch wood cores. 65 Figure 4.2 Top 15 compounds contributing to the separation of samples into treatment groups in PLSDA. 65

Figure 4.3 Volcano plot of compounds present in samples intersecting interaction zone lines compared with samples intersecting single colonies in attached branches. .66 Figure 5.1 PLS-DA model of Exidia glandulosa metabolome following primary and secondary resource capture. 77 Figure 5.2 Volcano plot of Exidia glandulosa metabolome following secondary resource capture compared with primary resource capture. 78

Figure 5.3 PLS-DA model of Exidia glandulosa metabolome following primary and secondary resource capture along xylem vessels.. 79 Figure 5.4 One-way ANOVA of the metabolome of Exidia glandulosa following primary resource capture and secondary resource capture from Oudemansiella mucida and Stereum rugosum along the wood vessels.

Figure 5.5 PLS-DA model of Exidia glandulosa metabolome following primary and secondary resource capture across xylem vessels. 
Figure 5.6 One-way ANOVA of the metabolome of Exidia glandulosa following primary resource capture and secondary resource capture from Oudemansiella mucida and Stereum rugosum along the wood vessels.

Figure 5.7 Interactive effect of initial colonising species and vessel orientation on the metabolome of Exidia glandulosa. 82

Figure 5.8 Interactive effects of early-arriving species and wood vessel orientation on the metabolome of Exidia glandulosa assessed by two-way between samples ANOVA. 83

Figure 5.9 Enzyme commission codes mapped to the proteome of Exidia glandulosa. 87 Figure 5.10 KEGG pathway analysis of the proteomoe of Exidia glandulosa following primary and secondary resource capture. 88

Figure 6.1 PLS-DA of the metabolome of Oudemansiella mucida under stable ad variable temperature regimes. 99

Figure 6.2 Volcano plot of Oudemansiella mucida metabolome under variable climate temperature compared with stable temperature 99

Figure 6.3 PLS-DA of the metabolome of Exidia glandulosa under stable ad variable temperature regimes. 101

Figure 6.4 Volcano plot of Exidia glandulosa metabolome under variable climate temperature compared with stable temperature 


\title{
Abbreviations
}

\author{
ACN - Aceto-nitrile \\ AMC - 7-amino-4-methyl coumarin \\ CAZymes - Carbohydrate active enzymes \\ CWD - Coarse woody debris \\ dbRDA - distance-based redundancy analysis \\ distLM - Distance-based linear modelling \\ DMAB - 3-(dimethylamino) benzoic acid \\ DMSO - Dimethyl sulfoxide \\ DNA - Deoxyribonucleic acid \\ EDTA - Ethylenediaminetetraacetic acid \\ FC - Fold change \\ FDR - False discovery rate \\ FWD - Fine woody debris \\ GC - Gas chromatography \\ GMD - Golm metabolome database \\ IS - Integrated signal \\ ITS - Internal transcribed spacer \\ JGI - The Joint Genome Institute \\ LC - Liquid chromatography \\ $\mathrm{LiP}$ - Lignin peroxidase \\ MBTH - 3-methyl-2-benzothiazoline hydrozone \\ MEA - Malt-extract agar \\ MiP - Manganese-independent peroxidase \\ $\mathrm{MnP}$ - Manganese peroxidase \\ MS - Mass spectrophotometry \\ MSTFA+ 1\% TMCS - N-Methyl-N-(trimethylsilyl) trifluoroacetamide \\ MUF - 4-methylumbelliferone \\ NAG - N-acetyl-glucosaminidase \\ NGS - Next-generation sequencing \\ NIST - National Institute of Standards and Technology \\ nMDS - Non-metric multidimensional scaling \\ OTU - Operational taxonomic unit \\ PCR - Polymerase chain reaction \\ PLS-DA - Partial least squares - discrimination analysis \\ PVP - Polyvinylpyrrolidone \\ qPCR - Quantitative real-time polymerase chain reaction \\ RNA - Ribonucleic acid \\ RSS - Random stratified sampling \\ RT - Retention time \\ SAR - Species-area relationship \\ VIP - Variable importance in projection
}




\section{Chapter 1 - General introduction}

\subsection{Importance of dead wood and dead attached branches in temperate woodland ecosystems}

\section{Contribution of dead attached branches to nutrient cycling}

Dead wood is an important constituent of temperate woodland systems with a volume of between 8 and $20 \mathrm{~m}^{3} \mathrm{ha}^{-1}$ arriving on the woodland floor every year (Forest Europe 2012). Dead attached branches are the major source of this newly-fallen wood, most of which is already well-decayed before reaching the ground (Boddy 2001; Boddy et al. 2017). In mature stands, there can be as much dead wood present in the system as dead attached branches as there is downed dead wood of all kinds, i.e. coarse- and finewoody debris (CWD; FWD respectively) and whole dead trees combined (Svensson et al. 2014).

The carbon in deadwood is difficult to access due to its high lignin content which gives wood its supportive structure (Sarkanen \& Ludwig 1971; Krankina et al. 1999; Kopra \& Fyles 2005; Schwarze 2007). At any given time, approximately $8 \%$ of the carbon present in global forests is locked in dead wood (Pan et al. 2011). As such, dead attached branches are a major component of one of the most important carbon and nutrient pools in land-based systems (Floudas et al. 2012; Rajala et al. 2012). Dead wood systems are also an important source of habitat for a wide range of organisms (Lonsdale et al. 2008; Rondeux \& Sanchez 2010; Lassauce et al. 2011) and contribute to the generation and stability of woodland soils (Kraigher et al. 2002; Debeljak 2006).

\section{Contribution of ligninolytic fungi to decomposition processes}

The decomposition of dead wood is effected by communities of ligninolytic fungi that develop in wood and which are the sole organisms capable of the complete reduction of lignin to monomeric compounds (Eriksson et al. 1990; Hibbett et al. 2007; Floudas et al. 2012; Doddapaneni et al. 2013). As such, any abiotic factors found to influence decomposition rates do so through effects exerted upon these fungal decomposers by altering their interactions with each other and their substrata (Boddy 2001; Weedon et al. 2009; Freschert et al. 2014). Indeed, the identity of decomposer organisms and their interactions may account for up to $73 \%$ of observed variation in decomposition rates (Bradford et al. 2014).

A central goal of microbial ecology is to understand the diversity and distribution patterns of communities (Purahong et al. 2018). These data are crucial for developing our understanding of the functional roles played by microbes in maintaining stability within ecosystems (Kubartová et al. 2012). While microbes have routinely been utilised as tools in the search for answers to questions about physiology and genetics, their communities have much more rarely been studied in nature or used as model systems for the controlled study of ecological community dynamics (Jessup et al. 2004). The 
conservation status of microbes in general, and particularly of ligninolytic and other wood-inhabiting fungi, has become an issue of concern in recent years due to the potential for cascading effects on diversity at higher taxonomic levels (Seibold et al. 2015).

\section{Current state of knowledge}

Current knowledge about ligninolytic fungal communities is highly fragmented however (Purahong et al. 2018) and that of communities associated with attached dead branches even more so. In fact, reviews by Boddy (2001; Boddy et al. 2017) illustrate that our entire understanding of the structure and general ecology of these communities in nature rests on a small number of papers published between 1983 and 1998 (Boddy \& Rayner 1983a, b; Boddy et al. 1987; Chapela \& Boddy 1988b; Boddy \& Griffith 1989; Hirst 1995; Hendry et al. 1998), all of which recorded the species richness of ligninolytic communities in branches of native species of angiosperm trees. The diversity and structure of communities of fungal fruiting bodies associated with attached angiosperm tree branches in relation to the diversity of microhabitats have also been reported (Unterseher et al. 2005; Unterseher \& Tal 2006) however these results were not related to the mycelia which drive decomposition processes.

\section{Expected contribution of this thesis}

There is clear evidence then that ligninolytic communities associated with dead attached branches contribute substantially to the turnover of resources in woodland systems due to their frequent occurrence and the extent of wood decomposition they effect. Despite this there have been no studies explicitly examining their community structure, taking into account species relative abundance as well as richness, nor of the factors that may influence their natural distribution patterns. This thesis sets out to shine a light on these forgotten engineers of woodland ecosystems by examining their community structure.

\subsection{The assembly and structure of ligninolytic fungal communities in dead attached branches}

\section{Community assembly in ligninolytic systems}

Ecological communities establish and assemble through the sequential and repeated immigration of species from the overall regional species pool (Fukami et al. 2010; Fukami 2015). In ligninolytic communities, species immigrations into woody substrata may occur through the dispersal of spores or, on the ground, the spread of mycelia between resources (Boddy et al. 2017). The diversity and structure of ecological communities is influenced by the order in which species immigrations occur (Dickie et al. 2011; Tan et al. 2012; Ottosson et al. 2014; Fukami 2015), including the order of arrival of the pioneer community (Frisch \& Green 2007; Louette \& De Meester 2007; Louette et al. 2008; Allen et al. 2011; Audet et al. 2013). It is known that the identity and abundance of the first-arriving species may either inhibit or facilitate the establishment success of later-arriving species through priority effects, although the 
mechanisms of these effects are not yet understood (Dickie et al. 2011; Hanson et al. 2012; Fukami 2015; Leopold et al. 2017).

Priority effects have been demonstrated in dead wood systems (Leather et al. 2014; Ottosson et al. 2014; Hiscox et al. 2015a, 2016b), indicating that the structure of the pioneer community, and potentially the order of arrival of pioneer species, will be an important factor in shaping the structure of later successional stages.

Attached dead branches and the primary succession in ligninolytic systems

The arrival of early colonists may be influenced by dispersal limitations (Finlay 2002; Dickie et al. 2011) and by environmental filtering which modifies community composition because under specific conditions the occurrence of specific traits, and therefore species identity, is constrained due to reduced competitive ability (Keddy 1992). In dead wood systems, particularly attached branches, inhibitory priority effects may be particularly strong due to niche pre-emption (Fukami 2015): species traits overlap significantly and the amount of resource available to other species is quickly limited by the extensive colonies produced by the pioneer community (Boddy 2001; Boddy et al. 2017).

The primary decay community establishes from latent propagules distributed throughout the recentlydysfunctional wood. Niche pre-emption in attached branch communities is facilitated by the presence of propagules of pioneer ligninolytic fungi within these endophytic populations. These pioneer species are capable of withstanding high water potential, with associated low $\mathrm{O}_{2}$, and high levels of toxic products of plant secondary metabolism (Rayner \& Boddy 1988; Boddy \& Griffith 1989; Boddy 2001; Boddy et al. 2017); conditions that are similar, although not quite as extreme, as those under active water transport in functional wood and which prevent colonisation of live sapwood by saprotrophs (Boddy 2001; Boddy et al. 2017).

The propagules of ligninolytic fungi are now known to be present asymptomatically as spores or mycelial fragments in the xylem between the cells of all healthy wood (Hirst 1995; Parfitt et al. 2010; Song et al. 2016). This endophytic community is thought to be dominated by non-wood decaying taxa however (Boddy et al. 2017), indicating that the dispersal processes involved may be passive, possibly linked to the transport of water within the tree.

Ligninolysis is an aerobic process driven largely by oxidative enzymes (Baldrian 2008). While the mechanisms governing endophyte identity and dispersal within wood are likely to be largely stochastic (Rodriguez et al. 2009; Boddy et al. 2017), the pioneer community are specialists of these previously described abiotic conditions. When drying of the wood begins, large colonies of pioneer species are often produced through primary resource capture when multiple genetically compatible propagules distributed throughout a branch are rapidly connected by the fusing of vegetative mycelia (Boddy 2001). 
Fallen dead wood and the secondary succession in ligninolytic systems

Ligninolytic fungi associated with later successional stages are known to have high combative ability and therefore to be able to effect rapid secondary recapture of resource from pioneers through the production of enzymes and inhibitory secondary metabolites (Boddy 2000; Hiscox et al. 2010a, 2018; Hiscox \& Boddy 2017). This process of invasion happens rapidly in woody debris resulting from the felling of healthy branches, wherein the pioneer community contributes minimally to overall decomposition processes which is thought to be a function of more rapid drying of the wood (Boddy 2001; Boddy et al. 2017).

\section{Exclusion of the secondary succession from attached dead branches}

In dead attached branches, invasion by combative secondary colonists is thought to be impeded by a combination of factors: high abiotic stress associated with high water potential (Heilmann-Clausen \& Boddy 2008); active defensive mechanisms employed by pioneer fungi such as the formation of thickly aggregated mycelia, known as pseudo-sclerotial plates, which physically impede invasive mycelia (Boddy et al. 2017); and the fact that secondary colonists must invade suitably dry wood from highly localised infections, such as wind-dispersed spores or pervasive endophytic propagules, giving the established pioneer species a distinct home advantage due to their large size (Viana et al. 2016; O'Leary et al. 2018). For these reasons, the primary successional stage is confined almost exclusively to dead attached branches while secondary and tertiary successions are excluded from dead attached branches and are therefore associated only with woody debris.

\section{Primary and secondary resource capture in ligninolytic communities}

The pioneer community fully colonises newly dysfunctional substrata through primary resource capture and any new species immigrating into the community must do so through secondary resource capture by displacement of all or part of the earlier colonists. Partial or complete replacement interactions may occur between pioneer fungi if the profile of abiotic stress changes sufficiently to give an advantage to a particular species in the community or species pool (Boddy \& Heilmann-Clausen 2008).

Species associated with the secondary succession are considered to be specialists of combative antagonistic interspecies interactions, however (Boddy 2000). Diversity and coexistence within natural secondary successional communities is thought to be maintained by the need to defend and invade space in three dimensions which is energetically expensive (Hiscox \& Boddy 2017; Hiscox et al. 2017) and through intransitivity in their relative combative abilities whereby multi-species interaction outcomes may follow a rock-paper-scissors pattern (Boddy et al. 2017; Kiziridis et al. 2018). In pioneer communities however, genetically-incompatible individuals are thought to be more defensive during biotic interactions with each other and instead, species coexistence is likely maintained due environmental rather than biotic selective pressures. 
Ligninolytic communities and the priority effect

The structure and order of arrival of the pioneer wood decay community, which is highly restricted to dead attached tree branches in natural woodland systems, will influence the trajectory of community assembly in later successional stages on the woodland floor. The outcome of these priority effects on later-stage community structure has been the central focus when exploring priority effects in ligninolytic communities (Weslien et al. 2011; Ottosson et al. 2014; Hiscox et al. 2015a, 2016b).

The underlying mechanisms driving priority effects from within the pioneer community are not yet understood and have rarely been explored. A major challenge is that it is very difficult to account for the historical arrival order of the pioneer community when surveying later stages and therefore to disentangle priority effects from environmental filtering and other influences on assembly processes (Fukami \& Morin 2003; Viana et al. 2016).

Model microbial communities can be a powerful tool facilitating the exploration of the genetic and biochemical traits underpinning complex ecological processes (Jessup et al. 2004). While ligninolytic fungi have proven to be a useful model system for exploring the impacts of priority effects on ecosystem functioning (Fukami et al. 2010), information pertaining to the stages of wood decomposition with which ligninolytic communities are associated in natural woodland systems is needed to interpret the underlying mechanisms driving those effects (Allen et al. 2000).

\section{Expected contribution of this thesis}

A principle aim of this thesis was to begin the process of exploring priority effects from within the context of the pioneer ligninolytic community with a view to providing comment on some of the potential biochemical mechanisms underpinning them and to facilitate future interpretation of their consequences on community assembly trajectories.

\subsection{The challenge of measuring fungal abundance in attached dead branches}

Presently available options for examining fungal community structure

Identification and quantification of the fungi present within natural substrata remains a major challenge in fungal ecology (Lindahl \& Boberg 2008; Kõljalg et al. 2013). The fungal diversity of natural wood decay systems has previously been reported using three principal approaches: culturing of mycelial individuals (papers reviewed within Boddy 2001; Boddy et al. 2017); surveys of fungal sporocarps (Bader et al. 1995; Renvall 1995; Lindblad 1998; Heilmann-Clausen \& Christensen 2004; Unterseher et al. 2005; Heilmann-Clausen et al. 2014); and genotype level descriptions of operational taxonomic units often produced by high-throughput sequencing of the non-coding internal transcribed spacer (ITS) DNA that sits between the 18S and 28S genes in eukaryotes (Kubartová et al. 2012; Schoch et al. 2012; Runnel et al. 2015; Hoppe et al. 2016). Fungal sporocarp surveys give some indication of the species that might be 
present within the wood but, given that some species will fruit only sporadically and sporocarp production within species varies between years, surveys must span several decades to provide a detailed picture of community diversity (Straatsma et al. 2001).

The challenge of quantifying fungal abundance in attached dead branches

Next-generation sequencing (NGS) efforts, such as those supported by Illumina and 454 pyro-sequencer platforms, provide rich datasets very rapidly. Within recently dysfunctional wood however, remnants of the endophyte community likely remain and NGS has previously revealed that in live sapwood this community is dominated by general, non-wood-decay taxa (Boddy et al. 2017). Thus, the exploration of diversity in the active ligninolytic community in attached dead branches seems an unsuitable subject for these highly sensitive approaches. Nevertheless, DNA sequencing approaches hold an advantage over isolation methods because the latter may discriminate against taxa that are non-culturable or slowgrowing (Lindahl \& Boberg 2008). However both high and low-throughput DNA sequencing involves multiple stages of DNA extraction, amplification, sequencing and bioinformatics; all of which may introduce bias and error into estimates of species richness and abundance (Carlsen et al. 2012; Nguyen et al. 2014; Oliver et al. 2015; Taylor et al. 2016). While quantitative real-time polymerase chain reaction (qPCR) is capable of quantifying fungal biomass in environmental samples (Liu et al. 2012), pioneer wood decay fungi produce extensive colonies in three dimensions within branches (Boddy 2001) which would be difficult to characterise through qPCR.

The importance of spatial dynamics in ligninolytic communities

One of the most important dynamics in ligninolytic communities in terms of resistance to biotic invasion and maintenance of diversity is territory within the spatially defined substratum. Competition for resource in these spatially defined substrata is synonymous with competition for space (Boddy 2000) and the spatial configuration of the early-stage community, over and above species identity and abundance, influences the assembly trajectory of the community in later stages (Toljander et al. 2006; Falconer et al. 2011). Furthermore the relative space each colony occupies within the substratum can destabilise competitive hierarchies, thereby maintaining community diversity (Hiscox et al. 2017; O'Leary et al. 2018).

\section{Expected contribution of this thesis}

A primary aim of this thesis was to apply a new approach to describe fungal relative abundance in attached dead branches; one that would take into account the relative size of mycelial individuals in three dimensions and take advantage of the strengths of both isolation techniques and DNA sequencing. 


\subsection{Fungal secondary metabolism and enzymatic decomposition of dead wood}

The potential of mass-spectrometry supported discovery of microbial metabolic responses

As chemo-heterotrophs, fungi interact with their environment and sequester nutrients through secondary metabolic processes and the secretion of enzymes, other proteins and low molecular weight bioactive compounds into their substrate (Keller et al. 2005; Brakhage 2013). These metabolic products are known to be utilised by ligninolytic fungi in the invasion and degradation of the structural components of wood cell walls (lignin, cellulose and hemicellulose) as their major source of carbon (Higuchi 1997; Lynd et al. 2002). However they are also used by ligninolytic fungi as chemical communication signals; in defending and invading territory; in buffering against unfavourable abiotic conditions; and in dealing with the toxic consequences of these interactions (Boddy 2000; Criquet et al. 2000; Baldrian 2004; D’Annibale et al. 2005; Yim et al. 2007; Brakhage \& Schroeckh 2011; Hiscox \& Boddy 2017; Moody et al. 2018a).

The mass-spectrophotometry (MS) supported detection of any differential diversity and abundance in the proteins and metabolites secreted by ligninolytic fungi under different conditions is a powerful tool being increasingly employed to developing understanding of fungal physiological responses and interactions (Peiris et al. 2008; Miyauchi et al. 2017; Moody et al. 2018a; O'Leary 2018; Zhong et al. 2018). Furthermore, assays of the potential activity levels of selected enzymes in environmental and experimental samples are capable of rapidly and cost-effectively providing more specific functional information (Sinsabaugh et al. 1993; Sinsabaugh 1994; Baldrian 2009).

Types of wood decay and ligninolytic fungi

The wood decay system overall is known to be highly biodiverse (Stockland et al. 2012). This diversity has been driven by co-evolution between woody plant species and microbes, resulting in a diversity of both the biochemical structure of plant lignins and the functional machinery employed by ligninolytic fungi to degrade them (Schwarze et al. 2000; Schwarze 2007). These combinations lead to a diversity in the types of wood decay that are loosely defined by the terms brown- white- and soft-rots.

This classification system describes the visual appearance of the decaying wood but not necessarily the phylogenetic relationships between, or the genetic machinery used by, the fungi that give rise to them (Eastwood et al. 2011; Riley et al. 2014). Nevertheless, the vast majority of fungi causing decay in the sapwood of angiospermous trees are considered to cause white-rots (Schultz \& Nicholas 2000) due to their enzymatic ability to degrade the lignin, as well as the cellulose and hemicellulose, component of the wood cell walls (Baldrian 2008).

Only 6-7\% of all ligninolytic fungi are known to cause brown-rot (Schwarze et al. 2000; Baldrian 2008). These are exclusively members of the phylum Basidiomycota and are largely restricted to gymnosperm 
tree species (Hibbett et al. 2001) due to the structural simplicity of the xylem and phloem which facilitates access to the cellulose and hemicellulose by brown-rot fungi following sequestration of cell wall-bound iron through the release of oxalic acids (Stockland et al. 2012). As such, the polyphyletic white-rot fungi are the only organisms truly capable of degrading lignin to its monomeric compounds (Eriksson et al. 1990; Hibbett et al. 2007; Eastwood et al. 2011; Floudas et al. 2012; Doddapaneni et al. 2013). Important white-rot fungi at all successional stages include members of the orders Polyporales, Russulales and Xylariales (Boddy \& Heilmann-Clausen 2008; Stockland et al. 2012).

\section{The structure of lignin in relation to wood cells}

Lignin, which makes up to $40 \%$ of wood mass, is an aromatic polymer that binds other cell-wall components together, confers to wood it's structure and is made up of three phenyl-based monomeric units in different ratios depending on the tree species: $p$-cumaril-alcohol, coniferyl and synapyl alcohol (Schwarze et al. 2000). Cellulose comprises long chains of hydroxyl-linked glucose monomers and constitutes up to $50 \%$ of wood mass (Nevell \& Zeronian 1985). Hemicellulose, together with lignin, forms a matrix within which the cellulose is bound. The most abundant angiospermous hemicelluloses are glucuronoxylan and glucomannan which comprise the cyclic sugars $\beta$-D-Glucopyranose, $\beta$-Dxylopyranose, $\beta$-D-mannopyranose along with 4-O-methylglucopyranosyluronic acid and acetyl side groups (Pettersen 1984).

Wood cell walls are essentially made up of three layers of cellulose bound within a matrix of lignin and hemicellulose (lignocellulose). The cellulose is arranged in helically wound microfibrils which are oriented tangentially to each other in the different layers of the cell wall. This complicated structure, together with the ether linkages and carbon-carbon bonds that hold the lignin polymer together, confer recalcitrance to the wood and lead to the generally slow rate of wood decomposition (Schwarze et al. 2000; Schwarze 2007) as well as the variety of metabolically-mediated strategies developed by fungi to circumnavigate them (Eastwood et al. 2011; Riley et al. 2014).

\section{Fungal-fungal and fungal-wood interactions}

The differential expression and activity levels of secreted enzymes and secondary metabolites by ligninolytic fungi have been studied from the perspective of combative interactions and mainly from the perspective of secondary-successional species (Hiscox \& Boddy 2017 and papers reviewed within). Combative interactions are not considered to be a predominant feature of pioneer communities in attached branches however. Pioneer species secure territory predominantly through primary resource capture and lack combative ability, tending to 'deadlock' with their neighbours once the whole substratum has been colonised (Boddy 2000; Boddy et al. 2017). An exception to this might follow a sharp change in the gradient of abiotic conditions, e.g. a prolonged period of desiccation, which would confer advantage to any highly stress-tolerant species in a given community affording them a chance to Page | 8 
become more dominant (Heilmann-Clausen \& Boddy 2008). Consequently, enzyme activity and the expression of proteins and metabolites are likely to differ in attached dead wood as a consequence, not of combat, but of different strategies employed by pioneer fungi in the search for nutrients and in coping with both subtle and drastic changes in abiotic conditions.

\section{Expected contribution of this thesis}

Understanding the functional mechanisms and consequences of these fungus-environment interactions is of ecosystem-scale significance given the role ligninolytic fungi play in biogeochemical processes. The functional biochemistry of ligninolytic fungi has never been studied from the perspective of the pioneer community. As such, another major aim of this thesis was to explore the responses of pioneer fungi, within microcosms and natural communities, at the protein and metabolite levels.

\subsection{Statement of thesis aims and research objectives}

This thesis describes a study which set out to explore pioneer wood decay communities associated with attached dead branches and the metabolic processes that govern pioneer fungal interactions during decomposition. Traditionally, wood decay communities and interactions have been studied from the perspective of communities and fungi associated with the secondary and, to a lesser extent, tertiary successions. Substantial wood decomposition is effected by the primary decay communities in nature however, and their structure, configuration and assembly may profoundly affect ecological processes in later successional stages. Through the application of surveying methods novel to wood decay systems, this study set out to provide a measure of mycelial relative abundance and the relative size of decay regions produce in attached branches and through applying enzyme assays and untargeted metabolomics and proteomics, to determine how metabolic processes are shaped by the biotic and abiotic interactions of pioneer fungi. Overarching hypotheses which have driven investigations are:

1. Pioneer ligninolytic community structure is shaped mainly by purely spatial factors rather than deterministic environmental processes,

2. Naturally formed pioneer communities will exhibit increased species richness with increased wood volume,

3. Metabolic processes in pioneer decay communities are linked to biotic interactions between species, variable abiotic conditions and with the gradient of decomposition, and

4. Priority effects exerted by early-arriving species on later-arriving species can be observed at the protein and metabolite level.

In order to test these hypotheses, a combination of observational and experimental approaches were applied to fungi associated with attached dead branches of beech (Fagus sylvatica). Specifically, the objectives of this work are: 
1. To explore the community structure and spatial patterns of naturally formed pioneer communities in attached F. sylvatica branches (Chapter 3),

2. To explore the metabolism of naturally-interacting pioneer fungi in F. sylvatica over a decomposition gradient (Chapter 4),

3. To explore the metabolism of naturally-interacting pioneer fungi in F. sylvatica in different regions of their decay columns relative to the interaction zone lines between them (Chapter 4),

4. To investigate the impact of variable temperature on the metabolism of a stress-selected (Exidia glandulosa) and non stress-selected (Oudemansiella mucida) pioneer fungus in microcosms, supported by genomes available through the Joint Genome Institute (JGI; Chapter 5), and

5. To investigate the long-term impact of earlier-arriving species (O. mucida and Stereum rugosum) on the metabolism of a later-arriving pioneer species (E. glandulosa) (Chapter 6). For this work, O. mucida was chosen because it is commonly isolated from natural pioneer communities and is known to be highly defensive in its competitive strategy while S. ugosum was chosen as a commonly isolated species with no such known attributes. E. glandulosa was selected as the later arriving species due to the availability of its genome through JGI and its stress-selected strategy which allowed controlled manipulation of its competitive success against the early-arriving species. 


\section{Chapter 2 - Materials and methods}

\subsection{Sampling methods employed in attached branches to characterise fungal communities}

\subsubsection{Study site}

Attached branch communities were surveyed from beech stands within Clyne Valley Woodlands, a minimally managed woodland in Swansea, South Wales UK (Figure 2.1). The site comprises 280 hectares of oak, birch and beech woodland stands, meadows and scrub habitat. Much of the woodland is impenetrable due to a thick bramble layer. However, stands to the north, north-east and centre (around Keeper's Cottage; Figure 2.1) of the site comprised numerous old beech trees and a sufficiently open structure to allow thorough surveying of the trees. An interactive version of the location of branches surveyed can be permanently accessed at https://goo.gl/6FWBxD.

\subsubsection{Selection of dead attached branches and generation of sampling locations along branch length}

Dead attached branches were located, geotagged and selected for sampling following extensive surveying of beech stands within Clyne Valley Woodlands (Figure 2.1; Section 2.1.1) undertaken seasonally. Branches were selected from the seasonally generated sampling pools using randomly generated numbers and removed with a $6.3 \mathrm{~m}$ telescopic pole saw (Cutting Edge Ltd). The length of the main branch was measured from the cut end to either the distal end of the branch or to the point at the distal end where branch diameter was $<1 \mathrm{~cm}$. Random sampling locations, one for each $50 \mathrm{~cm}$ of branch length, were generated and marked along the length of the branch. The distance $(\mathrm{cm})$ of these sampling locations from the cut end of the branch were used as a description of the spatial location of slice samples within branches ( $z$ distance) Where secondary branches were present, sampling continued in a parallel fashion with the main branch (Figure 2.1).

\subsubsection{Calculation of branch volume and lateral surface area}

The length and maximal and minimal diameters of the main branch (Fig. 2.2) were used to calculate branch volume $\left(V_{B}\right)$ as a truncated cone: $V_{B}=\frac{1}{3} \pi\left(r_{1}^{2}+r_{1} r_{2+} r_{2}^{2}\right) h$ where $h$ was the length, $r_{1}$ the maximal radius and $r_{2+}$ the minimal radius of the main branch. Volumes of significant secondary branches were combined additively with the volume of the main branch stem. The lateral area $(F)$ of the main branch was similarly calculated as $F=\pi\left(r_{1}+r_{2}\right) \sqrt{\left(r_{1}+r_{2}\right)^{2}+h^{2}}$. These measures were chosen due to the ease and efficiency with which they could be applied in the field. 


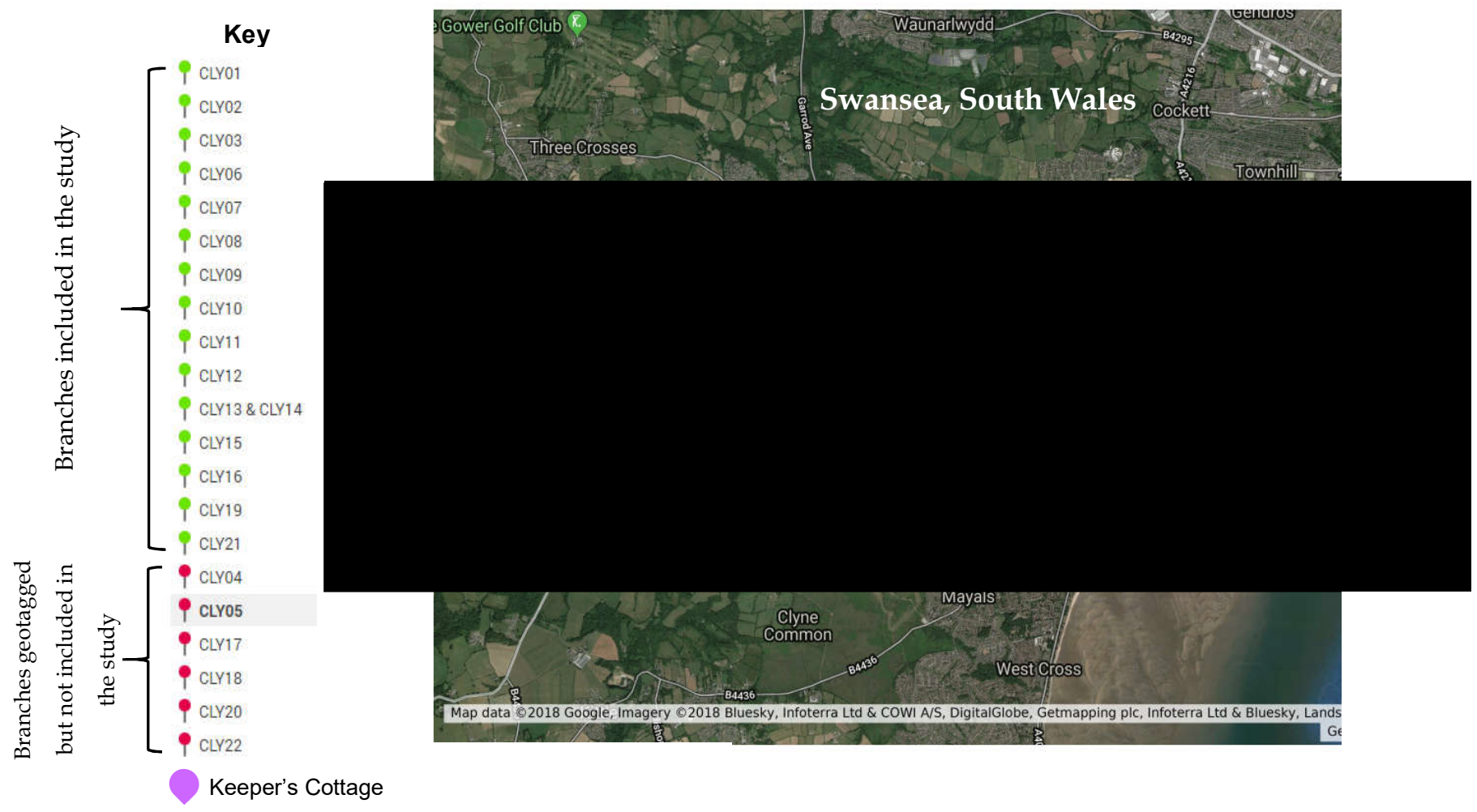

Figure 2.1 Location of attached dead branches at Clyne Valley Woodlands study site in Swansea, South Wales, UK
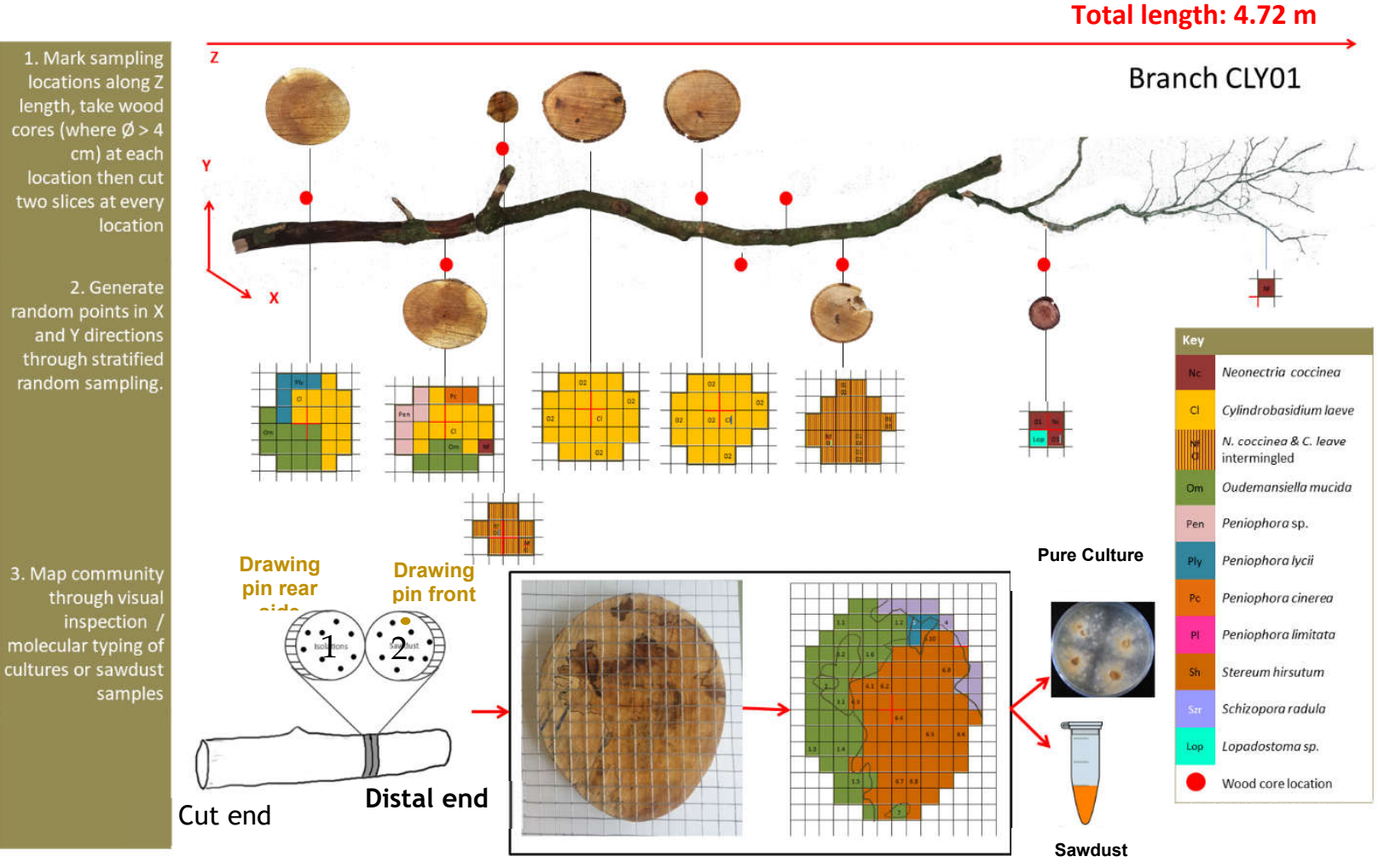

Figure 2.2 Sampling methods employed in attached branches to characterise fungal communities.

Red dots leading from branches indicate wood core extraction locations (Section 2.1.3); slices numbered 1 and 2 related to slices for wood chip isolations and sawdust sampling (Table 2.1); cut end of branch = closest to tree trunk; distal end - farthest from trunk; $\mathrm{z}$ direction $=$ longitudinal direction; $\mathrm{x}$ and $\mathrm{y}$ relate to the transverse plane of each wood slice 


\subsubsection{Extraction of branch wood cores for analyses of enzyme activity and metabolome profiles}

Where diameter of the branch $\geq 4 \mathrm{~cm}$, wood cores of length approximately equal to branch radius were extracted for analysis of enzyme activity and profiling of the metabolome. Cores were extracted from the branch at alternate marked sampling locations using an increment borer with a $5.15 \mathrm{~mm}$ auger diameter (Haglöf, Sweden). The orientation of entry into the branch was determined through random draws of four digits, each representing a different quadrant of the branch in transverse section (Figure 2.3). Cores were flash frozen in liquid nitrogen, freeze dried under vacuum for 24 hours and frozen (-80 ${ }^{\circ} \mathrm{C}$ ) for later analysis of enzyme activities, community proteome and community metabolome. Prior to extraction of extracellular proteins or metabolites, cores were split along their length to provide $50 \%$ of each sample for each procedure.

\subsubsection{Dissection of branches into slices for community structure mapping}

Following core extraction, branches were dissected into slices at each marked sampling location. Two slices (Table 2.1), approximately $2-5 \mathrm{~cm}$ thick depending on branch diameter, were extracted one from either side of each marked line. The orientation of each slice was marked by way of placing a drawing pin into the wood at the location denoting the region of wood consistent with the top of the branch facing (Fig. 2.2 and 2.3) and the face of the slice oriented in the trunk-ward direction (cut end; Fig. 2.2). From each pair, the slice farthest from the cut end was placed into a labelled sample bag and frozen (-20 ${ }^{\circ} \mathrm{C}$ ) for later analysis of DNA directly from wood samples (Section 2.2.3) while the slice nearest the cutend was placed into a labelled sample bag and incubated $\left(4^{\circ} \mathrm{C}\right)$ for a maximum of 24 hours prior to taking wood chip isolations from each decay region.

\subsubsection{Random stratified sampling, wood chip isolations and culture technique for identification of fungi resident in branches}

Culture technique was used to obtain pure cultures of fungi from each decay region from the face of Slice 1 without a pin (Fig. 2.2 and Table 2.1). The bark was stripped from each slice using a sterile chisel to allow surface sterilisation of all decaying sapwood surfaces and minimise the risk of isolating cultures of environmental contaminants that may be present between the bark and sapwood. The slice was then weighed to record the wet weight (Section 2.1.7) and then surface sterilised through submersion in a 1:10 solution of domestic bleach : distilled water. 


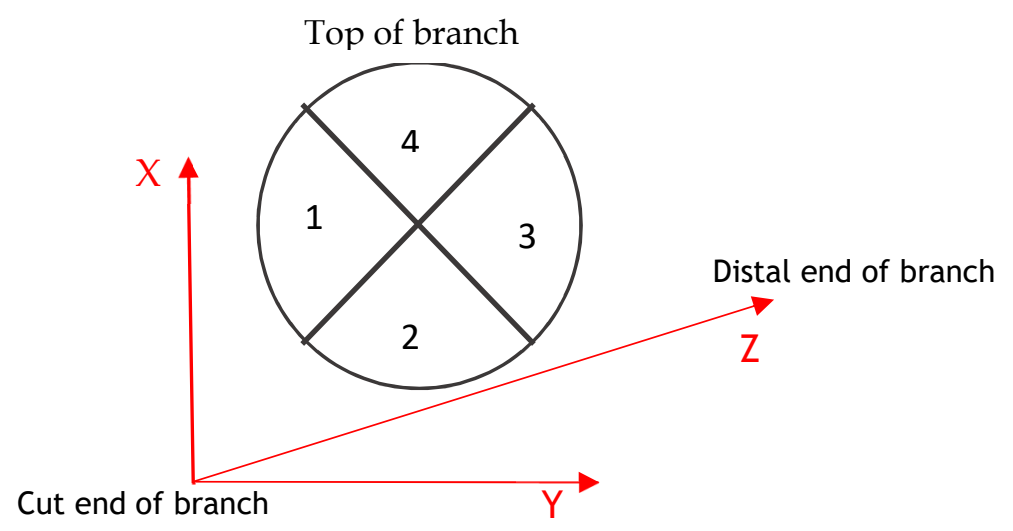

Figure 2.3 Strategy employed to determine orientation of extraction of wood cores for enzyme assays and metabolome profiling.

Transverse section of branch is shown in relation to the dimensions of the whole branch $(x, y$ and $z$ directions). Sampling locations were marked along the branch in the $\mathrm{z}$ direction and at each location where $\varnothing>4 \mathrm{~cm}$, a number between 1 and 4 was randomly drawn to determine the direction the increment borer would be inserted relative to $\mathrm{x}$ and $\mathrm{y}$. Locations 1 and 3 always related to the same side of the branch relative to the tree trunk.

Table 2.1 Summary of branch dissection regime for mapping the structure of attached branch communities

\begin{tabular}{llll}
\hline Slice Number & Location & Purpose & Samples taken from \\
\hline 1 & Nearer trunk & $\begin{array}{l}\text { Isolation of pure cultures - } \\
\text { wood chips }\end{array}$ & Face without pin \\
2 & Farther from trunk & $\begin{array}{l}\text { Direct DNA analysis - } \\
\text { sawdust }\end{array}$ & Face with pin \\
\hline
\end{tabular}
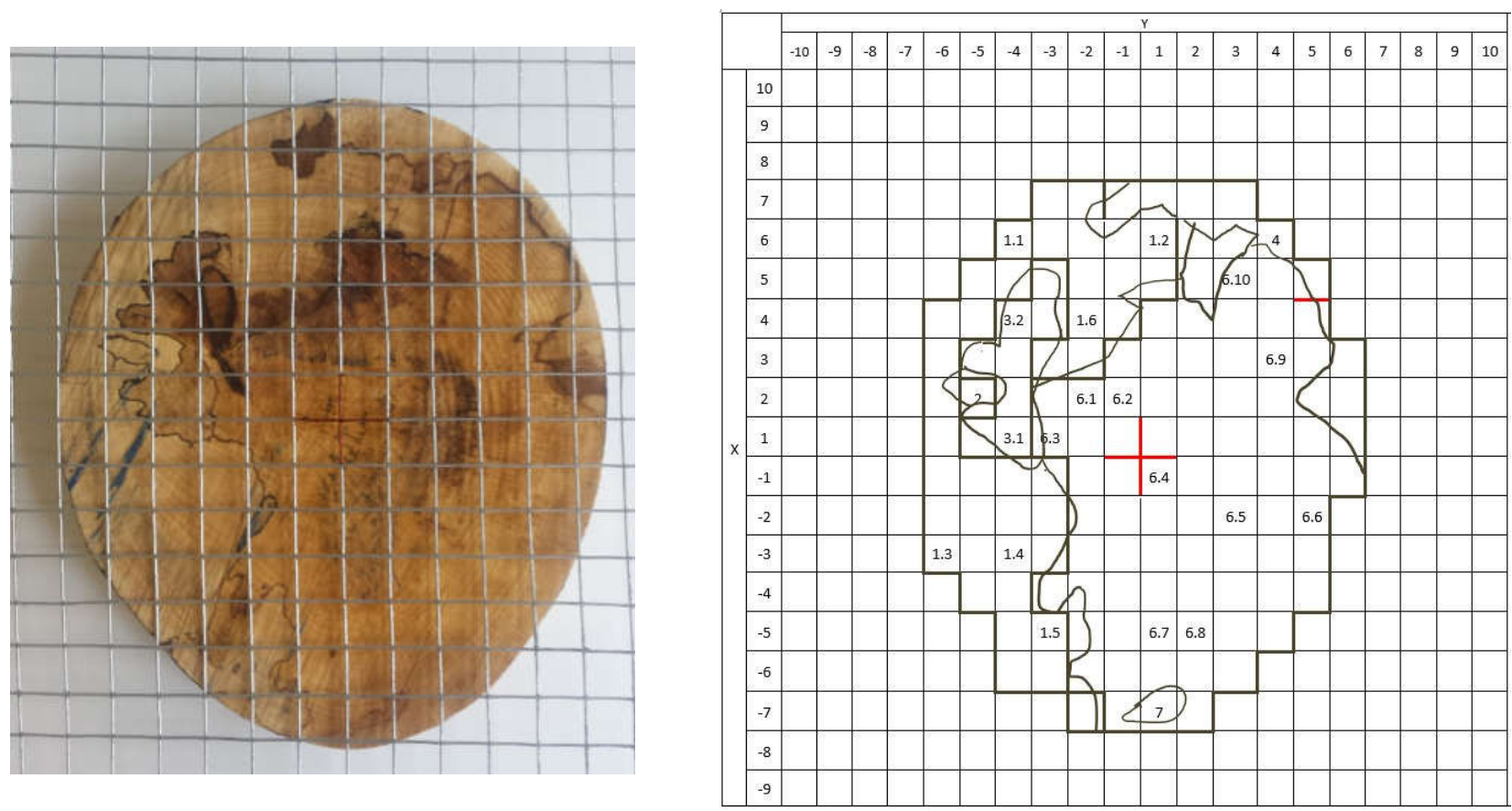

Figure 2.4 Applying random stratified sampling to wood slices.

(a) shows the placement of wire grid over the wood slice and the relative position of interaction zone lines in relation to each grid cell; (b) shows the recording matrix for the same wood slice with hand-drawn interaction zone lines. Spaces between zone lines = decay regions / strata; bold cell = where matrix cells were occupied by two different decay regions, the region occupying $>50 \%$ of the cell was deemed to have captured that cell; Numbers inside strata show the randomly generated sampling locations for each stratum where wood chip isolations were taken; If sawdust was needed from slice 2, the configuration shown above applied in mirror image by comparison with photographs and recording matrices and with the same wire grid placed centrally over the wood. 
To determine where wood chip isolations should be made in each decay region, a random stratified sampling (RSS) method was used. A $20 \times 20 \mathrm{~cm}$ wire grid (hole size $1.3 \mathrm{~cm}^{2}$ ) with the centre point marked was placed centrally over the wood slice and the configuration of decay zone lines was copied onto a gridded recording sheet (Fig. 2.4). Stratified sampling designs such as RSS partition the survey area into non-overlapping strata and data are collected from a randomly distributed set of locations within each stratum to reduce bias. Strata are sampled in proportion with their size, thus sampling effort in the present context is proportional to colony.

In keeping with these requirements, the total number of cells $(M)$ in each decay region were counted and a true random number $(0-M)$ generated as a seed. Counting top left to bottom right the seed cell was marked. The remaining sample locations within the stratum were identified by counting and marking every $n^{\text {th }}$ cell, both backwards and forwards, using randomly drawn numbers of between 1 and $M$ from the seed cell and successive sampling locations. Additional sample points were subjectively marked, highlighted on the recording sheet in a different colour, in locations where more information was needed to clarify the delineation between decay regions. As much randomness as possible was introduced into the study, by randomly choosing the locations in $\mathrm{x}, \mathrm{y}$ and $\mathrm{z}$ directions that all samples should be taken, to partially mitigate the effect of inevitable pseudo-replication arising from sampling cellular individuals belonging the same decay region in several slices within the same branch.

The resulting diagrams were used to inform the removal of wood chip isolations from just below the cut surface of the wood. All sampling locations marked on the recording sheet were subsequently marked in the relevant cell of the wire grid on the surface of the wood slice. The grid was then removed and, using a sterile $3 \mathrm{~mm}$ straight edge carving chisel (Jackson's Art Supplies, UK) and sterile forceps, the surface wood at each marked location was removed and discarded. Following tool re-sterilisation, another wood chip ( $3 \mathrm{~mm} \times 3 \mathrm{~mm}$ ) was removed from the newly exposed wood and plated onto $2 \%$ malt extract agar (MEA). Multiple chips from the same decay region were isolated onto the same plate and stored at $20^{\circ} \mathrm{C}$. Isolations were sub-cultured as needed to obtain pure cultures where possible. In some instances, multiple fungi grew out from the same wood chips indicating that their mycelia may have been intermingling in the wood. In these instances, each were sub-cultured onto separate plates to encourage them to develop in pure culture. Unless one or more species was determined to be an environmental contaminant introduced during culturing all species were recorded as inhabiting the relevant decay region and marked as such on branch community maps (Appendix I). Where pure cultures were produced, species were identified either through comparison of growth and macro characteristics with known cultures, including some supplied by the Cardiff Fungal Ecology Culture Collection, or through DNA sequencing (Section 2.2.1 and 2.2.2). 


\subsubsection{Calculation of wood density and dry mass loss for branch slices and whole branches}

Following extraction of wood chip isolations, any live sapwood was carefully removed from around the decaying wood of each slice. The outer dimensions of each slice were measured, and slices were placed in a drying oven $\left(100^{\circ} \mathrm{C}\right)$ for 72 hours and then weighed. The density $\left(D ; \mathrm{g} \mathrm{cm}^{-3}\right)$ of the dried wood was

calculated as $D=\frac{W_{d}}{V_{S}}$ where $W_{d}$ is the dry weight and $V_{S}$ is the slice volume calculated as $V_{S}=\pi r^{2} h$. The calculated density of wood in each slice was further converted to dry mass loss (\%) by comparison with the density of healthy sapwood taken to be $0.72 \mathrm{~g} \mathrm{~cm}^{-3}$ (Schwarze 2007). Averaged wood density and mass loss for each branch were calculated from $\sum V_{S}$ and $\sum W_{d}$ for all slices surveyed within a branch.

\subsection{Identification of fungi resident in the decay regions of attached branches}

\subsubsection{Preparation of pure cultures for DNA extraction}

Where pure cultures were produced but an identification could not be made from macroscopic characteristics shared with previously identified (known) cultures, identification was facilitated by sequencing of genomic DNA (gDNA). Many of the fungi isolated from tree branches grew predominantly in the form of submerged hyphae. To avoid introducing substantial amounts of MEA into preparations, cultures were first prepared by sub-culturing onto cellophane discs placed over $0.5 \%$ MEA which encouraged rapid growth. Once the culture had extended to $\sim 5 \mathrm{~cm} \varnothing$, the mycelia were transferred into a microcentrifuge tube, flash frozen in liquid nitrogen, freeze dried for 24 hours, and stored at $-20^{\circ} \mathrm{C}$. An aliquot of $<50 \mathrm{mg}$ tissue was used for DNA extraction.

\subsubsection{Extraction, amplification and sequencing of genomic DNA from pure cultures}

Total gDNA was extracted using the DNeasy Plant MiniKit (Quiagen) and assessed for quality through spectral patterns and 260/230 ratio values determined by spectrophotometery (Thermo). The ITS region of the nuclear ribosomal DNA was targeted and amplified by polymerase chain reaction (PCR) with two sets of published primers (Table 2.2). PCR reactions were performed in $20 \mu$ l volumes consisting of $1 \mu l$ of each primer, $4 \mu \mathrm{l}$ gDNA sample and $10 \mu \mathrm{l}$ Jumpstart RedTAQ readymix (Sigma-Aldrich). PCR cycling conditions were as follows: one cycle of $94^{\circ} \mathrm{C}$ for $2 \mathrm{~min}$ followed by 35 cycles of $94^{\circ} \mathrm{C}$ for $30 \mathrm{sec}$, annealing temperature (Table 2.2) for $30 \mathrm{sec}, 72^{\circ} \mathrm{C}$ for $5 \mathrm{~min}$. These conditions were followed by one cycle of $72^{\circ} \mathrm{C}$ for $5 \mathrm{~min}$ and then held at $10^{\circ} \mathrm{C}$ prior to storage at $-20^{\circ} \mathrm{C}$.

Samples were initially amplified using primers designed to target Basidiomycota. PCR products were visualised using gel electrophoresis. Agarose gels were made using $1 \%$ agarose gel and $0.001 \%$ SYBR safe in 1 x TAE buffer ( $\mathrm{pH}$ 8.3). Wells were loaded with either $5 \mu 100$ bp ladder (Promega) or $10 \mu \mathrm{l}$ PCR 
product and the gel run at 100v for approximately 30 minutes. Excised bands were purified using the Wizard SV Gel and PCR Clean-Up System (Promega). If the PCR failed, as indicated by the absence of a gel electrophoresis band, PCR was repeated using the Ascomycota-targeted primer set (Table 2.2). Purified PCR products were sequenced by Eurofins (Germany).

Table 2.2 Primers used for amplification of phylum-specific fungal ribosomal RNA genes.

Forward primers are 5' primers and reverse primers are 3' primers; sequences are written 5' - 3'; 1, (White et al. 1990); 2, (Larena et al. 1999); 3, (White et al. 1990)

\begin{tabular}{llllll}
\hline Primer & Targed phylum & Prime & Sequence & $\begin{array}{l}\text { Paired } \\
\text { primer }\end{array}$ & Ref \\
& & & & & \\
\hline ITS1-OF & Basidiomycota & $5^{\prime}$ & AACTCGGCCATTTAGAGGAAGT & ITS4-OF & 1 \\
ITS4-OF & Basididiomycota & $3^{\prime}$ & GTTACTAGGGGAATCCTTGTT & ITS1-OF & 1 \\
ITS5 & Ascomycota & $5^{\prime}$ & GGAAGTAAAAGTCGTAACAAGG & ITS4A & 2 \\
ITS4A & Ascomycota & $3^{\prime}$ & CGCCGTTACTGGGGCAATCCCTG & ITS5 & 3 \\
\hline
\end{tabular}

\subsubsection{Extraction of sawdust for fungal genomic DNA analysis}

In cases where no pure culture could be obtained from wood chip isolations, identifications of fungi present in decay regions were made by extraction of total gDNA directly from sawdust. To achieve this, the pin-marked face of Slice 2 (Table 2.1) was covered by the same $20 \times 20 \mathrm{~cm}$ wire grid as used previously (Section 2.1.5) was placed centrally over the wood slice (Fig. 2.4) and the configuration of interaction zone lines was compared with the mirror images from Slice 2 (Table 2.1) recorded as photographs and RSS sample location recording sheets (Section 2.1.5). The appropriate sampling locations were marked on the surface of the wood and the wire grid removed. Sawdust was then extracted using a $4.5 \mathrm{~mm}$ drill bit with the wood slice secured over a sterile plastic collection chamber. This facilitated rapid transference into a microcentrifuge tube with minimal risk of contamination by airborne spores. Samples were flash frozen in liquid nitrogen, freeze dried for 24 hours and stored at $-20^{\circ} \mathrm{C}$.

\subsubsection{Extraction of total gDNA from sawdust samples}

An aliquot of sawdust weighing < $500 \mathrm{mg}$ was used for crude extraction of total gDNA. Sawdust samples were vortexed with three sterile steel beads for 30 seconds, incubated in $400 \mu 11 \times$ TE buffer (pH 8.0 ) at $70^{\circ} \mathrm{C}$ for 2 hours, centrifuged for 10 minutes, $17,000 \mathrm{~g}$. The supernatant was drawn off the pellet and stored at $-20^{\circ} \mathrm{C}$. Prior to amplification by PCR, crude gDNA extractions were cleaned using the PowerClean Pro DNA Clean-UP kit (MoBio). This protocol was chosen for extraction of DNA from sawdust because commercially available extraction kits (Qiagen, Germany; Cambio, UK) routinely resulted in very low total gDNA yield $\left(<10 \mathrm{ng} \mathrm{ml}^{-1}\right)$ only a small proportion of which would likely be of fungal origin. In addition, all gDNA samples extracted from sawdust exhibited signs of significant carry over of polysaccharides as indicated by spectral patterns and 260/230 ratio values determined by spectrophotometery and which regularly inhibited the PCR. Where gDNA yield was already very low, 
templates could not be cleaned of inhibitors and still be viable for amplification by PCR. As such, this protocol of extracting crude DNA templates following mechanical lysis of the plant and fungal tissues was developed and this produced consistently higher yields of gDNA with fewer problems associated with PCR inhibition.

\subsubsection{Amplification of gDNA extracted from sawdust samples}

Following gDNA extraction from sawdust samples, the ITS region of fungal ribosomal DNA was again amplified by primers designed to target fungi resident in the wood belonging to either the Basidiomycota or the Ascomycota (Table 2.2). It was recognised that multiple fungi may be present within the same wood samples and that primers would likely amplify the DNA of the species with greater biomass or affinity to the primer sequence. As such, sawdust samples were routinely amplified by both sets of primers in an attempt to document the community as fully as possible. PCR reactions were performed in $50 \mu \mathrm{l}$ volumes consisting of $2.5 \mu \mathrm{l}$ of each primer, $10 \mu \mathrm{l}$ gDNA sample and $25 \mu \mathrm{l}$ Jumpstart RedTAQ ready mix (Sigma-Aldrich). PCR cycling conditions were as follows: one cycle of $94^{\circ} \mathrm{C}$ for $2 \mathrm{~min}$ followed by 35 cycles of $94^{\circ} \mathrm{C}$ for $30 \mathrm{sec}$, annealing temperature (Table 2.2) for $30 \mathrm{sec}, 72^{\circ} \mathrm{C}$ for $5 \mathrm{~min}$. These conditions were followed by one cycle of $72^{\circ} \mathrm{C}$ for $5 \mathrm{~min}$ and then held at $10^{\circ} \mathrm{C}$ prior to storage at $-20^{\circ} \mathrm{C}$. PCR products were visualised using gel electrophoresis. Agarose gels were made using $5 \%$ agarose gel and $0.001 \%$ SYBR safe in $1 \times$ TAE buffer (ph 8.3). Wells were loaded with either $5 \mu 100$ bp ladder (Promega) or $10 \mu \mathrm{l}$ PCR product and the gel run at $100 \mathrm{v}$ for approximately 30 minutes. Excised bands were purified using the Wizard SV Gel and PCR Clean-Up System (Promega). Purified PCR products were Sanger sequenced by Eurofins (Germany).

\subsubsection{Resolving the inhibition of PCR amplification of $\mathrm{gDNA}$ extracted from sawdust samples}

Despite the development of gDNA extraction procedures outlined in 2.2.5, there were rare instances where PCR amplification produced poor results as indicated by very feint gel electrophoresis bands or the complete absence of bands. In addition, a small number of samples produced multiple products that were hard to separate manually through cutting of the individual bands. In the case of single feint PCR bands, PCR products were directly purified using the Wizard SV Gel and PCR Clean-Up System and Sanger sequenced. Where multiple products were produced, samples were run through higher percentage $(10 \%)$ gels at a lower voltage to encourage better separation of products. In some cases, it was only possible to successfully excise one of the products. Where no gel electrophoresis band was produced, polyvinylpyrrolidone (PVP) was added directly into the PCR reaction mixture (Section 2.2.5) at a final concentration of $1 \%\left(\mathrm{v} / \mathrm{v}\right.$; Koonjul et al. 1999). PVP was stored in solution at $-20^{\circ} \mathrm{C}$. Once thawed, PVP solutions older than 6 days were discarded. PCR reactions were again performed in $50 \mu 1$ 
volumes consisting of $2.5 \mu \mathrm{l}$ of each primer, $10 \mu \mathrm{l}$ gDNA sample, $25 \mu \mathrm{l}$ Jumpstart RedTAQ ready mix (Sigma-Aldrich) and $10 \mu \mathrm{l}$ PVP.

\subsubsection{Bioinformatics of sequences offungal $\mathrm{gDN} A$}

Sequence files provided by Eurofins (TubeSeq Sanger Service) were imported into Geneious R 9.0.5. Read direction of ITS4 and ITS4A sequences was set to reverse and a global alignment of all sequence reads was undertaken using the following parameters: global alignment with free end gaps; cost matrix with $65 \%$ similarity (5.0/4.0); gap open penalty of 12 ; gap extension penalty of 3 . Aligned sequences were trimmed of the relevant primers. Chromatograms of aligned sequences were then manually inspected to resolve base ambiguities wherever possible. Consensus sequences were generated using a $50 \%$ (strict) threshold and exported in FASTA format (Appendix I). Subsequently, Geneious was used to align consensus sequences with the NCBI nr database using BLASTn. Alignments with organisms with match quality $\geq 97 \%$ were recorded. The exported consensus sequences were further aligned against the UNITE database (Nilsson et al. 2018) using massBLASTer. The UNITE databse is populated with wellannotated records of fungal ITS sequences from well-defined herbarium specimens and include full herbarium reference identification, collector/course and ecological metadata (Kõljalg et al. 2005) whereas metadata within NCBI are scant and quality control is generally low. Where results from high quality (match $\geq 97 \%$ ) alignment with the UNITE database conflicted with the results from the NCBI database, records were amended to reflect the results returned by UNITE.

\subsubsection{Measures and data analysis offungal species richness and diversity within branches}

Species richness within branches was determined by reference to branch community maps (Appendix I). Decay regions occupied by different species of fungi were counted as a measure of species richness. Multiple counts of the same species were made in situations where the same species appeared in the same branch community in more than one decay region, but those decay regions had no connectivity with each other, as denoted by the identities of species occupying branch slices between them. While species richness does not take into account the number of individuals of each species present, the biological species concept is difficult to apply to ligninolytic fungi wherein genetically incompatible colonies of the same species do not recognise each other as 'self' and so compete with each other for resource and for space (Boddy 2000; Maynard et al. 2017). It was not ascertained in the present study whether disconnected colonies of the same species within branches were genetically compatible or not and so all were deemed potential competitors and contributors to the richness of communities. Following this same principle, the number of cellular individuals $(n)$ was counted as the number of sampling cells (Section 2.1.5) within each decay region. Decay regions connected between adjacent slices in the $z$ direction by fungi of the same identity were considered to be occupying the same decay region. 
Thus, counts of individuals were also a proximate measure of colony size in three dimensions $(x, y, z$; Fig. 2.2). The number of neighbours a colony had within a branch was determined by reference to branch community maps and counting the number of non-self colonies bordering the colony of interest. Simpson's Index of Diversity (1-D), which considers both the richness and evenness of a community, was used as a measure of diversity and increases towards 1 as diversity increases. Simpsons's $D$ is

calculated as $D=\frac{\sum n(n-1)}{N(N-1)}$ where $N$ was taken as the total count of individuals of all species in a community, i.e. the total number of cells in a branch.

Statistical analyses were conducted using R statistical software (R Core Team 2018) in R Studio v1.1.456 (R Studio Team 2016). Species richness and diversity measures were tested for correlations with branch volume, branch mass loss, and branch lateral area using Spearman-rank correlation analysis. Statistically significant correlations $(\mathrm{P} \leq 0.05)$ where the null hypothesis of no correlation between dependent and independent variables was tested, were used to denote the presence of a SAR and correlation direction was used to determine whether any SAR present was positive or negative.

\subsubsection{Data analysis of fungal community structure within branches}

\subsubsection{Community structure data pre-treatment}

Community structure was considered at the slice rather than branch level to enable the investigation of branch physical and spatial characteristics on the identity and relative abundance of species. Analyses were conducting using PRIMER v7 (Clarke \& Gorley 2015) with add-on package PERMANOVA+ (Anderson et al. 2008a).

The abiotic dataset for branch slices comprised physical (environmental) parameters and spatial parameters. Physical parameters were: branch slice diameter $(\mathrm{cm})$; slice mass loss (\%); and host tree condition ('Alive') programmed as a dummy variable where $1=$ live tree and $0=$ dead tree. Spatial parameters were: the latitudinal geolocation of the branch (decimal degrees); the longitudinal geolocation of the branch (decimal degrees); and the distance $(z)$ of the slice from the cut end of the branch $(\mathrm{cm})$. In order to preserve the arrangement of slices within branches, $z$ distance values were programmed as decimal values. The integer-part of each decimal value comprised a sequential numerator for each branch (i.e. numbers 1-16) which were assigned irrespective of any spatial relationship between the branches or temporal relationship in terms of when the branches were surveyed in relation to each other. The fractional-part of each decimal number was the $\mathrm{z}$ distance value programmed as thousandths (i.e. three decimal places). This strategy ensured that in the resemblance matrix, slices closer to each other and in the same branch had greater resemblance than two slices with similar $z$ distance values from different branches. The spatial relationship between branches within the study site (Fig. 2.1) was preserved through the longitude and latitude values which would result in greater resemblance between branches that were attached to trees positioned closer together. Thus, 
spatial variation within and between branches was kept separate within multivariate models, allowing for the interpretation of either of these purely-spatial influences on community structure to be applied with confidence. By programming the randomly-generated $z$ distance variable as a spatial variable in models, the issue of unavoidable pseudo-replication of cellular individuals from the same decay column, which will not arise independently of each other, was partially addressed. Furthermore, by accounting for both within- and between-branch spatial aggregation, the true effect of branch physical parameters on the observed clustering of species and individuals within branches could be ascertained. Abiotic variables were examined for the absence of autocorrelation and outliers by way of draftsman plot. Variables were normalised but not transformed and the abiotic resemblance matrix built using Euclidean distance resemblance measures.

The biotic dataset comprised a site $\mathrm{x}$ species abundance matrix where sites were branch slices. The data set was $\log 1$ transformed following examination by shade plot (Clarke et al. 2014). Functional data (trophic mode, guild; Appendix I) were assigned to each species following literature searches and FUNGuild DB Query (Nguyen et al. 2016). Functional data were programmed as a qualitative traits $x$ species matrix and taxonomic levels deemed to be informative (species, order and phylum; Appendix I) were programmed as a qualitative taxon $x$ species matrix. These matrices were used to aggregate the biotic resemblance matrix for examination of effects at different taxonomic and functional levels. Biotic resemblance matrices were subsequently built using Bray-Curtis similarity resemblance measures. All proceeding analyses were conducted using the abiotic and biotic resemblance matrices.

\subsubsection{Unconstrained ordination of attached branch communities visualised by comparison with decay stage}

Analyses were undertaken in PRIMER 7. To visualise the degree to which community assembly structure varied at different stages of decomposition, the decay gradient ( $0-75 \%$; Table 3.1 was divided into equal thirds to represent the low- medium- and high-level of overall decomposition having taken place. An unconstrained model was built using non-metric multidimensional scaling (nMDS) with biotic and abiotic variables pooled by branch to better visualise the relative openness of communities associated with each third of the decay gradient. To visualise influence spatial variables and branch physical characteristics may have on the structure of communities and openness of communities undergoing similar extent of decomposition, the vectors the abiotic datasheet were overlaid on the nMDS ordination plot using Pearson correlations. To examine whether a simplified model could have sufficiently explained the variation in community structure and to examine which species were most important in driving the separation and clustering of branch communities within the nMDS model, BEST analysis was undertaken using step-wise searching and Bray-Curtis similarity measure (termination criterion $\rho>0.95$ ). 


\subsubsection{Variation partitioning of environmental and spatial effects on attached branch community structure}

Distance-based linear modelling (distLM) detects and quantifies relationships between a highly multivariate data cloud such as community structure, which comprises both species identity and abundance at different surveying sites, and one or more predictor variables (Legendre \& Anderson 1999; McArdle \& Anderson 2001). The predictor variable with the strongest relationship with, and therefore that explains the largest proportion of, variation in multivariate species abundance is fitted first. Subsequently, when employing step-wise selection, additional variables are either added or subtracted from the model in a manner that allows the model to explain greater and greater degrees of variation until the best model is found. Given that predictor variables will not be completely independent of each other there will be overlap in terms of the observed variation they explain. Under a true null hypothesis, once the relationship between the response data cloud and predictor variable $X_{1}$ has been dealt with, permuting the samples randomly would destroy the relationships between predictor variables $X_{1}$ and $X_{2}$ in the search for a relationship between predictor variable $X_{2}$ and the data cloud. This would result in the model ascribing too much independent influence to $X_{2}$ on the observed variation in community structure. To avoid this, when considering the null hypothesis of no linear relationship between $X_{2}$ and the observed variation in the data cloud, distLM uses permutation of residuals under a reduced model (Freedman \& Lane 1983; Anderson \& Legendre 1998). As such, with each permutation, the degree of variation already explained by previously fitted variables is always taken into account and the P-values of subsequently added (or subtracted) variables are based on whether they explain a large or small part of the remaining unexplained variation (Fig. 2.5). If marginal tests are run, these will test the null hypothesis of no relationship between the predictor variable and the total variation in the data cloud and interactions between predictor variables are ignored. As such, the same predictor variable may have a significant relationship

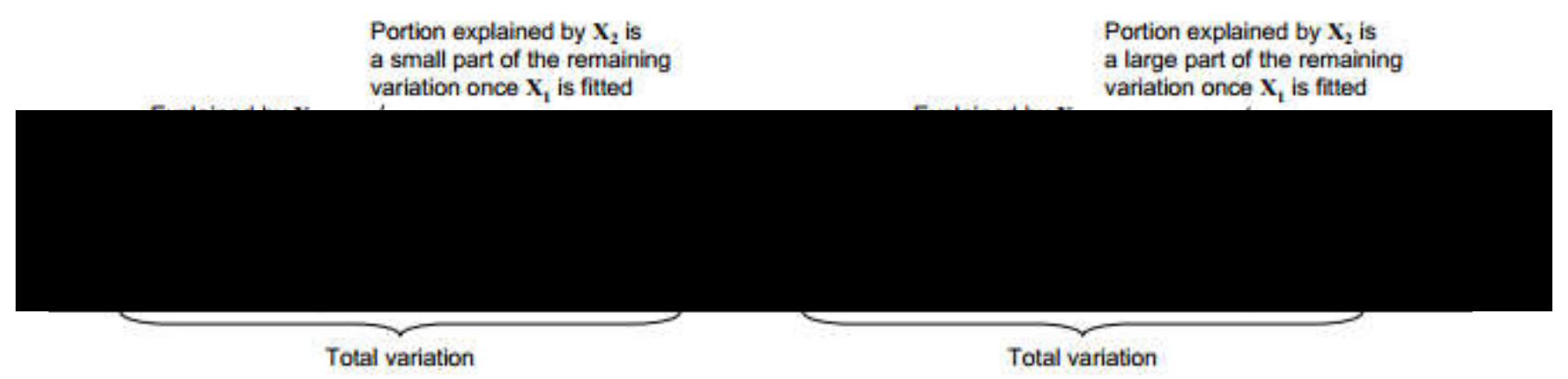

Figure 2.5 A two-dimensional representation of the observed variation in a community structure data cloud and the application of permutation of significant P-values of the predictor variables in a distLM model

Reproduced from (Anderson et al. 2008b). (a) illustrates the situation where a secondary predictor variable $X^{2}$ is found to be a significant explanator of community structure variation in marginal tests but not in the full distLM model. (b) illustrates the situation where a secondary predictor variable $\mathrm{X}^{2}$ does not explain a significant degree of variation in community structure in marginal tests, but contributes significantly to the full distLM model. Marginal tests assume no interaction between predictor variables and so the influence of each on community structure is considered in isolation of all other predictor variables. 
with the data cloud under marginal testing but contribute insignificantly to the distLM model (Fig. 2.5a) or the reverse may be true (Fig. 2.5b).

Normalised abiotic variables were set as either environmental (diameter, mass loss, tree alive) or spatial (latitude, longitude, $z$ ) indicators for variation partitioning. Distance-based linear models were subsequently built on resemblance matrices at each level of taxonomic and functional aggregation with the normalised abiotic variables set as predictor variables and the following parameters: predictor variables grouped by indicators; a step-wise selection procedure; adjusted $\mathrm{R}^{2}$ selection criterion; obtain P-values by 999 permutations; run marginal tests; and plot the model as a distance-based redundancy analysis (dbRDA) plot. Environmental or spatial partitions were deemed to have a greater effect on the observed variation in community structure if they were fitted into the model first and if they explained a greater proportion of observed variation in community structure than the other. Significance level was deemed to be $\mathrm{P} \leq 0.05$. Models tested the null hypothesis that there was no linear relationship between observed variation in community structure and variation in the partition under consideration. Under variation partitioning, if significant effects of the environmental partition on community structure are found, particularly if the partition is more important in explaining the variation in response than the spatial partition, confers greater confidence in interpreting any relationships found between individual environmental variables and community structure. Conversely, if the spatial partition is found to be more important in explaining variation in the system, relationships between individual environmental variables and community structure should be interpreted with caution due to the potential conflation of niche effects with spatial auto-correlation.

\subsubsection{Distance-based linear modelling of effects of predictor variables on attached branch community structure}

Distance-based linear models were subsequently built on resemblance matrices at each level of taxonomic and functional aggregation with the normalised abiotic variables set as predictor variables and the following parameters: no grouping of predictor variables by indicators; a step-wise selection procedure; adjusted $\mathrm{R}^{2}$ selection criterion; obtain P-values by 999 permutations; run marginal tests; and plot the model as a dbRDA plot. Significance level was deemed to be $\mathrm{P} \leq 0.05$. Models tested the null hypothesis that there was no linear relationship between observed variation in community structure and variation in the predictor variable under consideration.

\subsection{Measuring fungal enzyme activity levels in attached branches}

\subsubsection{Extraction of total protein from branch wood cores}

One half of the wood cores, previously prepared (Section 2.1.4), were manually chipped and shaken overnight at $150 \mathrm{rpm}$ and $4{ }^{\circ} \mathrm{C}$ in $30 \mathrm{ml} 50 \mathrm{mM}$ sodium acetate buffer (pH 5.2) with $0.05 \%$ Tween80. The 
buffer was kept on ice and filtered through sterile glass wool, Whatman filter paper (no. 1) and $0.2 \mu \mathrm{m}$ membrane filters for further refinement. The filtrate was then concentrated using centrifugal filter tubes with a 3k molecular weight cut-off (Merck Millipore), centrifuged at $3500 \mathrm{xg}$ and $4{ }^{\circ} \mathrm{C}$ until $3 \mathrm{ml}$ remained. Finally, the preparation was precipitated in 4 volumes of cold acetone and centrifuged at $10,000 \times \mathrm{g}$ for 60 minutes. The isolated protein pellet was resuspended in $500 \mu 150 \mathrm{mM}$ sodium acetate buffer (pH 5.2) with $10 \mu$ l protease inhibitor cocktail (Promega) and frozen $\left(-80^{\circ} \mathrm{C}\right)$. These methods were adapted from Dawson-Andoh \& Morell (1992) and Moody et al. (2018a) to ensure minimal cell breakage and targeting of the extracellular proteome whilst also ensuring high protein recovery.

\subsubsection{Bradford's assay and standardisation of protein concentrations across samples}

Protein concentration ( $\mu \mathrm{g} \mathrm{ml}^{-1}$ ) of each extract was measured by Bradford's Assay. Total protein in $150 \mu \mathrm{l}$ protein extract was calculated for each sample and all samples standardised to the concentration of sample CLY13C (55 $\mu \mathrm{g} \mathrm{ml}^{-1}$ ) with 50mM sodium acetate buffer ( $\mathrm{pH}$ 5.2) added to bring the final volume to $150 \mu \mathrm{l}$ (Table 2.3).

\subsubsection{Assays of activity level of hydrolytic enzymes}

The activities of the hydrolytic enzymes cellobiohydrolase, $\alpha$-glucosidase, $\beta$-glucosidase, $\beta$-xylosidase, $N$-acetyl-glucosaminidase (NAG), arylsulfatase, phosphomonesterase, phosphodiesterase, alanine aminopeptidase and leucine aminopeptidase were determined through fluorogenic microplate assays using 4-methylumbelliferone (MUF) and 7-amino-4-methyl coumarin (AMC) linked-substrates according to the methods established by Vepsäläinen et al (2001). Substrates were purchased from Sigma-Aldrich. Substrates and standards were prepared in $10 \mathrm{ml}$ dimethyl sulfoxide (DMSO). MUF and AMC stock solutions were further diluted to 1:10 (0.1 mM) and 1:100 (0.01 mM) working solutions prior to running the assays. All substrates were stored in dark vials and at $4{ }^{\circ} \mathrm{C}$ when not in use. Samples were diluted 1:100 in $50 \mathrm{mM}$ sodium acetate buffer $\left(\mathrm{pH}\right.$ 5.2) and stored at $-80{ }^{\circ} \mathrm{C}$ when not in use.

Fluorogenic assays were undertaken in black 96-well microplates (Greiner Bio-one) to prevent interference between samples. Each plate was set up to measure the activity of the ten hydrolytic enzymes over three technical replicates and standard curves (MUF and AMC), in the presence of the assayed sample under identical assay conditions, for two biological samples. All wells received $200 \mu \mathrm{l}$ of sample, which had previously been standardised (Section 2.3.2) and diluted as outlined above, and those assessing enzyme activities received $40 \mu \mathrm{l}$ of the respective substrate. In addition, $40 \mu \mathrm{DMSO}$ was added to wells assaying activity of $\beta$-glucosidase and phosphatase. 
Table 2.3 Protein concentrations calculated per total protein extraction from branch wood core samples by Bradford's assay. Standard curve was calculated by assaying bovine serum albumin standards with concentration 0, 50, 100, 200, 400, 600 and 800 $\mu \mathrm{g} \mathrm{ml}^{-1}$. To standardise all samples to the concentration of CLY13C which had the lowest concentration, the volume ( $\left.\mu \mathrm{l}\right)$ of sample stated in the righter-most column was brought to a final volume of $150 \mu 1$ with $50 \mathrm{mM}$ sodium acetate buffer (pH 5.2).

\begin{tabular}{lcc}
\hline Sample & Concentration, ${\mathbf{~ g ~} \mathbf{~ m l}^{-\mathbf{1}}}$ & Vol Sample, $\boldsymbol{\mu l}$ \\
\hline CLY13C & 55 & 150.00 \\
CLY03E & 68 & 121.48 \\
CLY04C & 76 & 107.74 \\
CLY04A & 77 & 106.53 \\
CLY19C & 91 & 90.28 \\
CLY03D & 96 & 86.16 \\
CLY04B & 98 & 83.85 \\
CLY19E & 122 & 67.49 \\
CLY07E & 127 & 64.67 \\
CLY07G & 131 & 62.91 \\
CLY12B & 133 & 62.06 \\
CLY03C & 139 & 59.27 \\
CLY19A & 149 & 55.34 \\
CLY13G & 187 & 44.03 \\
CLY04D & 208 & 39.54 \\
CLY03A & 209 & 39.36 \\
CLY13E & 230 & 35.84 \\
CLY03B & 240 & 34.29 \\
CLY07C & 388 & 21.26 \\
\hline
\end{tabular}

Curve calibration wells received volumes of MUF or AMC substrate stock or working solutions to obtain final concentrations of $0,0.4,0.8,1.6,8,16,40$ and $80 \mu \mathrm{M}$ or $0,0.08,0.16,0.4,0.8,1.6,4$ and $8 \mu \mathrm{M}$ respectively. Assays were undertaken at $40^{\circ} \mathrm{C}$ and fluorescence read after 5 and 125 minutes (excitation at $355 \mathrm{~nm}$, emission at $460 \mathrm{~nm}$ ) with a Biotek Synergy H1 multi reader. As such, a separate standard curve was calculated for each sample to account as much as possible for quenching of substrates (Poll et al. 2006; Kramer et al. 2013).

\subsubsection{Assays of activity levels of oxidative enzymes}

Activity of manganese peroxidases (MnP) was determined by following the oxidation of 3-methyl-2benzothiazoline hydrozone (MBTH) and 3-(dimethylamino) benzoic acid (DMAB) which results in a yellow product. The assay separately determines the activity of oxidases, peroxidases without $\mathrm{MnP}$ and MnP. $100 \mathrm{mM}$ phosphate buffer ( $\mathrm{pH}$ 6.5) was made by adding $0.95 \mathrm{~g} \mathrm{KH}_{2} \mathrm{PO}_{4}$ and $0.54 \mathrm{~g} \mathrm{Na}_{2} \mathrm{HPO}_{4} .2 \mathrm{H}_{2} \mathrm{O}$ to $100 \mathrm{ml}$ ultrapure water. $25 \mathrm{mM}$ DMAB was made by adding $0.0826 \mathrm{~g}$ DMAB to $20 \mathrm{ml}$ phosphate buffer (pH 6.5). $1 \mathrm{mM}$ MBTH was made by adding $0.0043 \mathrm{~g}$ MBTH to $20 \mathrm{ml}$ ultrapure water. $100 \mathrm{mM}$ succinate lactate buffer ( $\mathrm{pH} 4.5)$ was made by adding $0.146 \mathrm{~g}$ succinic acid and $1.64 \mathrm{~g}$ DL-lactic acid to $100 \mathrm{ml}$ ultrapure water. $2 \mathrm{mM}$ Ethylenediamine-tetra-acetic acid (EDTA) was made by adding $0.074 \mathrm{~g}$ $\mathrm{Na}_{2}$ EDTA. $2 \mathrm{H}_{2} \mathrm{O}$ to $50 \mathrm{ml}$ ultrapure water. Immediately prior to pipetting, $5 \mathrm{mM}$ hydrogen peroxide was 
prepared by adding $60 \mu \mathrm{l} 30 \% \mathrm{H}_{2} \mathrm{O}_{2}$ to $100 \mathrm{ml}$ ultrapure water. Reaction mixtures contained: $50 \mu \mathrm{l}$ sample (diluted as outlined above) and $200 \mu \mathrm{l}$ of either solutions AR, AP or AB (Table 2.4).

Table 2.4 Preparation of substrate solutions for quantitative fluorogenic assays of hydrolytic enzymes.

\begin{tabular}{lccc}
\hline & Substrate AR & Substrate AP & Substrate AB \\
\hline Succinate-lactate buffer $100 \mathrm{mM}$ & $150 \mu \mathrm{l}$ & $150 \mu \mathrm{l}$ & $150 \mu \mathrm{l}$ \\
DMAB $25 \mathrm{mM}$ & $20 \mu \mathrm{l}$ & $20 \mu \mathrm{l}$ & $20 \mu \mathrm{l}$ \\
$\mathrm{MBTH} 1 \mathrm{mM}$ & $10 \mu \mathrm{l}$ & $10 \mu \mathrm{l}$ & $10 \mu \mathrm{l}$ \\
$\mathrm{MnSO}_{4} 2 \mathrm{mM}$ & $10 \mu \mathrm{l}$ & - & - \\
$\mathrm{H}_{2} \mathrm{O}_{2}$ & $10 \mu \mathrm{l}$ & $10 \mu \mathrm{l}$ & - \\
EDTA $_{2} \mathrm{mM}$ & - & $10 \mu \mathrm{l}$ & $10 \mu \mathrm{l}$ \\
Ultrapure water & - & - & $10 \mu \mathrm{l}$ \\
\hline
\end{tabular}

The addition of EDTA to substrates $\mathrm{AP}$ and $\mathrm{AB}$ binds free $\mathrm{Mn}^{2+}$ so activity of $\mathrm{MnP}$ can be deduced by subtracting the activity of substrate $\mathrm{AB}$ from that of substrate AP. The addition of hydrogen peroxide to substrates AR and AP allows activity of peroxidases so that they can be distinguished from general oxidases which is denoted by the activity of substrate AB. Therefore, the activity of manganeseindependent peroxidases $(\mathrm{MiP})$ can be determined by subtraction of the activity of substrate $\mathrm{AB}$ from that of substrate AP. Three technical replicates were performed per sample per substrate solution. Blank and negative control wells received either $250 \mu \mathrm{l}$ acetate buffer in place of reaction mixtures or $50 \mu 1$ acetate buffer in place of biological samples respectively. Absorbance at $590 \mathrm{~nm}$ was measured immediately after pipetting and every 50 seconds for 15 minutes with a Biotek Synergy H1 multi reader.

Lignin peroxidase $(\mathrm{LiP})$ activity was determined by following the oxidation of veratryl alcohol in microplate assays according to the methods developed by Tien \& Kirk (1989). Reaction mixtures contained: $130 \mu \mathrm{l}$ sample (diluted as outlined above), $50 \mu \mathrm{l} 10 \mathrm{mM}$ veratryl alcohol (in $50 \mathrm{ml}$ ultrapure water), $50 \mu \mathrm{l} 250 \mathrm{mM}$ tartaric acid ( $\mathrm{pH}$ 2.5; in $250 \mathrm{ml}$ ultrapure water) and $20 \mu 15 \mathrm{mM}$ hydrogen peroxide (60 $\mu 130 \%$ hydrogen peroxide in $100 \mathrm{ml}$ ultrapure water, prepared immediately before use). Three technical replicates were performed per sample. Blank and negative control wells received either $250 \mu 1$ acetate buffer in place of reaction mixtures or $130 \mu \mathrm{l}$ acetate buffer in place of biological samples respectively. Absorbance at $310 \mathrm{~nm}$ was measured immediately after pipetting and every 50 seconds for 3 minutes $\left(\mathrm{E}=9300 \mathrm{M}^{-1} \mathrm{~cm}^{-1}\right)$ with a Biotek synergy $\mathrm{H} 1$ multi reader.

\subsubsection{Data analysis of enzyme activity levels of fungi in attached branch communities}

\subsubsection{Detecting differences in enzyme activity levels between competing and non-competing}

\section{fungi}

Samples were grouped into two groups: those colonised by two or more competing fungi and those colonised by a lone fungus. Grouping was aided by reference to branch community maps (Appendix I) 
on which the location and orientation of wood core extractions had been marked. Cores that had intersected one or more interaction zone lines between different fungi were included in the competing group. Cores that had intersected a single fungal colony were included in the non-competing group. Separation into the two groups was further aided by the visual appearance of interaction zone lines in the core samples.

All statistical analyses were conducted in R Studio. Mean enzyme activity levels from three technical replicates per sample were compared using Wilcoxon signed rank test which tested the null hypothesis of no significant differences in activity levels between the two groups.

\subsubsection{Detecting changes in fungal enzyme activity levels over gradients of branch and} community dynamics

Correlation analyses were used to determine whether changes in enzyme activity levels correlated with variation in the size of branches, the level of decomposition that had taken place, the size of the colonies producing the observed enzyme activity levels or the numbers of neighbours they had (Section 2.2.8).

All statistical analyses were conducted in R Studio. Spearman-rank correlations were calculated to test the null hypothesis of no correlation between the mean enzyme activity levels from three technical replicates per sample and the predictor variable of interest.

\subsection{Profiling the metabolome of fungi in attached branches}

\subsubsection{Extraction of fungal metabolites from branch wood cores}

One half of branch wood core samples, previously prepared (Section 2.1.4), were each manually chipped, added to $\leq 7 \mathrm{ml}$ aceto-nitrile $(\mathrm{ACN})$ : methanol : $\mathrm{H}_{2} \mathrm{O}(2: 2: 1)$ and shaken at $180 \mathrm{rpm}$ at room temperature for 1 hour. Extracts were filtered through Whatman filter paper (no. 1) and then centrifuged at 3,500 $\mathrm{g}$ for $30 \mathrm{~min}$. The supernatant was dried in vacuo (Thermo Savant) overnight.

Derivatization of dried samples was carried out by addition of $30 \mu 1$ methoxylamine hydrochloride (15 $\mathrm{mg} \mathrm{ml}^{-1}$ in pyridine) followed by incubation at $70^{\circ} \mathrm{C}$ for 60 minutes. Subsequently $50 \mu 1$ N-Methyl-N(trimethylsilyl) trifluoroacetamide (MSTFA+ 1\% TMCS; Thermo) was added to the sample and the resulting mixture incubated at $40^{\circ} \mathrm{C}$ for 90 minutes. Derivatised samples were transferred to autosampler vials and $10 \mu \mathrm{l}$ tetracosane $\left(5 \mathrm{mg} \mathrm{ml}^{-1}\right.$ in heptane) added as an internal standard.

\subsubsection{GC-MS analysis of metabolome profiles from branch wood cores}

$1 \mathrm{ul}$ of sample was injected onto a 5975 gas chromatography (GC)-MS (Agilent) with inert mass spectrometer detector. The column used was a 5\% Phenyl Methyl Siloxane column (30m * 250uM * $0.25 \mathrm{uM}$ ) with a helium carrier gas flow rate of $1 \mathrm{ml} / \mathrm{min}$. The injector was operated in splitless mode at a temperature of $250^{\circ} \mathrm{C}$ and the column oven temperature was held at $60^{\circ} \mathrm{C}$ for 1 minute before being 
increased at a rate of $10^{\circ} \mathrm{C}$ per minute until $180^{\circ} \mathrm{C}$ and then at $4^{\circ} \mathrm{C}$ until reaching $300^{\circ} \mathrm{C}$. The mass spectrometer utilised an electron emission of $70 \mathrm{eV}$ and scanned from 50-650 amu per scan.

\subsubsection{Deconvolution, pre-processing and alignment of GC-MS chromatograms}

GC-MS data were initially processed using MSD ChemStation software (E.02.02.1177) and chromatograms imported into AMDIS 2.70 (D’Arcy \& Mallard 2004) for deconvolution and comparison of deconvoluted spectra with the National Institute of Standards and Technology (NIST) 2011 library (Wiley) and Golm Metabolome Database mass spectral reference library (GMD; Kopka et al. 2005; Downloaded 13th September 2018). Putative identifications were based on matches of mass spectra ( $\geq$ 80\% similarity) and visual inspection of matched spectra in NIST MS Search 2.0 (Stein et al. 2011). Chromatograms were highly complex consisting of several thousand peaks per sample (Appendix II), a common feature of GC-MS analysis of complex mixtures from carbohydrates caused by the presence of multiple tautomeric forms of carbohydrate derivatives in solution (Ruiz-matute 2011). To aid the removal of internally-matching peaks and the alignment of chromatograms from different samples with each other, data pre-processing was undertaken by SpectConnect (Flory et al. 2007). SpectConnect is designed to reduce GC-MS datasets down to only those compounds conserved between replicates but in an experiment with lack of replication (e.g. Chapter 3) both conserved and unique compounds can be identified using SpectConnect. A huge advantage of applying SpectConnect following GC-MS analysis of complex carbohydrate mixtures is that it searches for and removes internally-matching peaks. Furthermore, the comparative difference in compound identities between samples, including rare compounds, was of interest. As such, the 12 chromatogram files were loaded into SpectConnect as pairs of samples under 6 conditions, irrespective of the design of the experiment. The elution threshold was set to $1 \mathrm{~min}$ and the support threshold was set to $50 \%$. This ensured that any compound appearing in only $50 \%$ of one condition pairing would be present in the resulting data matrices (i.e. singletons across the whole dataset would be reported), in addition to compounds conserved across at least any two samples. In addition, these were found to be the most conservative settings to align the peaks produced by the tetracosane internal standard as a single row in the output data matrices.

Compound abundances (concentrations) were calculated using the integrated signal (IS) matrix produced by SpectConnect. The matrix row corresponding to the tetracosane internal standard was identified and the IS of all other compounds in each column normalised that of the relevant internal standard. Compound abundances were then divided by half the volume $\left(\mathrm{cm}^{3}\right)$ of the wood core to give compound abundance values $\mathrm{cm}^{-3}$. Matrix rows were associated with their chromatogram peaks and putative compound identifications by cross-referencing the IS matrix with both the retention time (RT) matrix produced by SpectConnect and the retention times of peaks in the chromatograms in which the compound appeared within AMDIS. Common contaminants of GC-MS datasets (e.g. disiloxanes which are hydrolysed from MSTFA+ $1 \%$ TMCS on contact with $\mathrm{H}_{2} \mathrm{O}$ ) were removed from the datasets along Page | 28 
with chromatogram peaks that were found to comprise $<6$ mass spectra. All compounds (rows) in the final matrix (Appendix II) were numbered sequentially and unidentified compounds maintained these numbers as identifiers. Analytical compound names were cross referenced with the names of bioactive metabolites using GMD and other public repositories such as ChemSpider (Pence \& Williams 2010), PubChem (Wang et al. 2009) and the NIST Chemistry WebBook (Linstrom \& Mallard 2001) and analytes corresponding to the same metabolite were merged additively. Metabolites were assigned to biological functions using the GOLM Metabolome Database (Hummel et al. 2010) and KEGG Pathway (Kanehisa \& Goto 2000).

\subsubsection{Statistical analysis of profiles of fungal metabolites from branch wood cores}

\subsubsection{Processing and normalisation of attached branch community metabolome}

Data were uploaded to MetaboAnalyst 4.0 (Chong et al. 2018) as a sample x compound matrix using an un-paired design. Data filtering was not applied as the previous pre-processing and alignment steps had adequately removed variables likely to be non-informative. Data were normalised by median, g-log transformed and mean centred so that the dataset followed a near-normal distribution.

\subsubsection{Detecting differences in fungal metabolomes between competing and non-competing fungi}

Differential analysis of metabolome profiles of samples in the competing and non-competing groups were undertaken in MetaboAnalyst 4.0. Partial least squares - discriminant analysis (PLS-DA) was used to visualise whether the relative abundances of any compounds in the dataset were likely to result in the separation of samples into the two experimental groups within acceptable confidence limits (95\%).

Validation of the predictive accuracy of the model was calculated by cross-validation and inspection of Q2 values. Q2 values approaching 1 were taken as an indication of relatively strong predictive accuracy and negative values as an indication of over-fitted models, which would lead to a high probability that any separation of samples seen in the two-dimensional PLS-DA scores plot arose by chance. Compounds that contributed most, due to the relative concentrations in the two experimental groups, to the separation of samples into experimental treatment groups were identified by variable importance in projection (VIP) analysis. All compounds with VIP > 1.0 were considered to be 'important' compounds and therefore to be compounds that were likely to differ in concentration between the two conditions.

T-tests with a 5\% Benjamini-Hochberg false discovery rate (FDR) correction for multiple comparisons (Benjamini \& Hochberg 1995) were applied to test the null hypothesis of no significant differences in compound concentration between experimental conditions. 
2.4.4.3 Detecting changes in the concentration of selected metabolites over the decomposition gradient

Lipids are important metabolites for the storage of carbon and energy in all biological systems. In addition sugar alcohols have been found to be important during interspecific combative interactions between ligninolytic and other saprotrophic fungi (Hiscox \& Boddy 2017) and the availability of the major sources of carbon in decaying wood, such as cellulose, may change predictably as decomposition advances. The concentrations of metabolites identified from these chemical classes were examined over the decomposition gradient (average mass loss of wood within the branch). Statistical analyses were performed in R Studio. Spearman-rank correlations were computed to test the null hypothesis that no correlation was present between wood mass loss and the metabolite of interest.

\subsubsection{Distance-based linear modelling of effects of branch physical properties and fungal enzyme activity levels on the fungal metabolome in branch wood cores}

To determine whether wood physical properties, community dynamics or enzyme activity levels exhibited linear relationships with the observed variation in the metabolome of fungi in attached branch communities, datasets were loaded into PRIMER 7. The enzyme activity level dataset was loaded as an environmental data sheet. Variables were square root transformed and normalised and a resemblance matrix built using Euclidean distances. An environmental dataset comprising slice mass loss (\%), slice water content (\%), colony size $(n)$ of the fungi present in the wood core samples, number of neighbours held by the fungi present in the wood core samples and slice diameter $(\mathrm{cm})$ was loaded as an environmental data sheet (Section 2.2.8). Data were square root transformed and normalised. Finally, the normalised transformed metabolome dataset produced within MetaboAnalyst 4.0 (Section 2.4.4.2) was loaded into PRIMER 7 and a resemblance matrix built using Euclidean distances.

Models were built on resemblance matrices of the metabolome with either the branch environment or enzyme activity levels variables as predictor variables and the following parameters: no grouping of predictor variables by indicators; a step-wise selection procedure; adjusted $\mathrm{R}^{2}$ selection criterion; obtain P-values by 999 permutations; run marginal tests; and plot the model as a dbRDA plot. Significance level was deemed to be $\mathrm{P} \leq 0.05$. Models tested the null hypothesis that there was no linear relationship between observed variation in metabolite concentrations and variation in the predictor variable under consideration.

\subsection{Microcosm woodblock experiments}

\subsubsection{Fungal strains}

Fungal species isolated from attached branch communities (Section 2.1) were selected based on their common occurrence and ecological roles: Exidia glandulosa, considered to be a stress-selected species, Oudemansiella mucida, known to be a highly defensive strategist amongst pioneer fungi and Stereum Page | 30 
rugosum which was also a commonly isolated pioneer species. All strains were wild-type strains isolated from naturally colonised resources in the earlier work.

\subsubsection{Set-up, experimental design and interaction assembly of microcosm experiments}

Sterile beech (Fagus sylvatica) blocks with dimensions $2 \mathrm{~cm}^{3}$ were pre-colonised with fungi by placing on $0.5 \%(\mathrm{w} / \mathrm{v})$ MEA cultures of the appropriate fungal strain and incubated in the dark at $20^{\circ} \mathrm{C}$ for 8 weeks. Following pre-colonisation, mycelia and agar were scraped from the surface of each wood block using a flame-sterilised blade and forceps.

The pre- colonised wood blocks were combined in a $2 \times 2$ block matrix per microcosm, secured for the first 7 days with a sterile elastic band. Each pot had $4 x<1 \mathrm{~mm}$ holes covered with microporous tape for aeration. Matrices either comprised four blocks all colonised with the same species, or two pairs of blocks for pairwise species interactions.

Secured wood block matrices were placed into sterile polypropylene pots (100 ml; Cater4You Ltd) containing $2 \mathrm{~cm}$ sterile perlite. A volume of $10 \mathrm{ml}$ autoclaved distilled $\mathrm{H}_{2} \mathrm{O}$ was added to each pot prior to incubation. Pairwise interaction treatment groups (3 replicates of four treatment groups) were incubated at $15^{\circ} \mathrm{C}$ for 8 weeks.

Single-species configurations were colonised by either E. glandulosa or O. mucida and incubated either at $15^{\circ} \mathrm{C}$ (stable temperature) for 8 weeks or under a diurnal temperature regime where microcosms were held at $5^{\circ} \mathrm{C}, 15^{\circ} \mathrm{C}, 25^{\circ} \mathrm{C}$, then $15^{\circ} \mathrm{C}$ for 6 hours per temperature every 24 hours for 8 weeks. All four treatment groups (3 replicates per treatment) were used to examine the effect of variable temperature on the metabolome and proteome of E. glandulosa and O. mucida (Chapter 6). The treatment group colonised by E. glandulosa and held stable at $15^{\circ} \mathrm{C}$ was also used as the primary territory capture control group (3 replicates) for the examination of lasting effects of initial colonising species on the proteome and metabolome of E. glandulosa (Chapter 5).

Pairwise interactions were conducted in two orientations relative to xylem vessels: (1) with the blocks colonised by different species arranged cut end to end so that inter-species interactions took place along the length of the xylem vessels; and (2) with the blocks colonised by different species arranged with xylem vessels in parallel so that species interactions took place across the xylem vessels. Pairwise interaction treatment groups ( 3 replicates of four treatment groups) were incubated at $15^{\circ} \mathrm{C}$ for 8 weeks. These treatment groups, along with microcosms colonised entirely by E. glandulosa and held stable at $15^{\circ} \mathrm{C}$ (above), were used to examine lasting effects of initial colonising species and substrate complexity on the metabolome and proteome of E. glandulosa (Chapter 5). 


\subsubsection{Determination of completion of replacement interactions}

Following 8 weeks incubation each block in the $2 \times 2$ matrix of each replicate in all treatment groups were split into thirds along the grain using a sterile chisel. The top and middle sections were wrapped in foil, flash frozen in liquid nitrogen, freeze dried for 24 hours and stored at $-20^{\circ} \mathrm{C}$ ahead of being used for proteomic and metabolomic profiling. From the bottom third of each block, four wood chips measuring approximately $0.5 \times 0.5 \mathrm{~mm}$ were extracted, one from each quadrant, and placed onto $2 \%(\mathrm{w} / \mathrm{v}) \mathrm{MEA}$. Plates were incubated at $20^{\circ} \mathrm{C}$ for 7 - 10 days which allowed for morphological identification of the final species present in each block to ensure that replacement interactions were complete and blocks were colonised by pure cultures.

\subsection{Profiling the metabolome of fungi in beech woodblocks}

\subsubsection{Extraction of fungal metabolites from beech woodblocks}

The middle third of woodblocks from each treatment group (one per species per treatment group; Section 2.5.2) were each weighed and manually chipped, added to $\leq 10 \mathrm{ml}$ aceto-nitrile : methanol : $\mathrm{H}_{2} \mathrm{O}$ $(2: 2: 1)$ and shaken at $180 \mathrm{rpm}$ at room temperature for 1 hour. Extracts were filtered through Whatman filter paper (no. 1) and then centrifuged at 3,500 $\mathrm{g}$ for $30 \mathrm{~min}$. The supernatant was dried in vacuo (Thermo Savant) overnight.

Derivatization of dried samples was carried out by addition of $30 \mu 1$ methoxylamine hydrochloride (15 $\mathrm{mg} \mathrm{ml}^{-1}$ in pyridine) followed by incubation at $70^{\circ} \mathrm{C}$ for 60 minutes. Subsequently $50 \mu \mathrm{MSTFA}+1 \%$ TMCS (Thermo) was added to the sample and the resulting mixture incubated at $40^{\circ} \mathrm{C}$ for 90 minutes. Derivatised samples were transferred to autosampler vials and $10 \mu 1$ tetracosane $\left(5 \mathrm{mg} \mathrm{ml}^{-1}\right.$ in heptane) added as an internal standard.

\subsubsection{GC-MS analysis of metabolome profiles from woodblock samples}

1 ul of sample was injected onto a 5975 GC-MS (Agilent) with inert mass spectrometer detector. The column used was a 5\% Phenyl Methyl Siloxane column $(30 \mathrm{~m}$ * $250 \mathrm{uM}$ * $0.25 \mathrm{uM})$ with a helium carrier gas flow rate of $1 \mathrm{ml} / \mathrm{min}$. The injector was operated in splitless mode at a temperature of $250^{\circ} \mathrm{C}$ and the column oven temperature was held at $60^{\circ} \mathrm{C}$ for 1 minute before being increased at a rate of $10^{\circ} \mathrm{C}$ per minute until $180^{\circ} \mathrm{C}$ and then at $4^{\circ} \mathrm{C}$ until reaching $300^{\circ} \mathrm{C}$. The mass spectrometer utilised an electron emission of $70 \mathrm{eV}$ and scanned from $50-650$ amu per scan.

\subsubsection{Deconvolution, pre-processing and alignment of GC-MS chromatograms}

GC-MS data were initially produced using MSD ChemStation software (Agilent Technologies Ltd 2011) and chromatograms imported into AMDIS for deconvolution and comparison of deconvoluted spectra 
with the NIST 2011 and GMD mass spectral reference libraries(GMD; Kopka et al. 2005; Downloaded 13th September 2018). Putative identifications were based on matches of mass spectra ( $\geq 80 \%$ similarity) and visual inspection of matched spectra in NIST MS Search 2.0.

Data pre-processing was undertaken by SpectConnect with chromatogram files loaded according to the experimental designs of the experiments. For differential analysis of effects of temperature (Chapter 6), treatments colonised by E. glandulosa and O. mucida were handled as two separate experiments. Chromatograms were uploaded as 3 replicates in two temperature treatment groups, repeated for each species.

For differential analysis of the effect of an initial colonising species and interaction orientation on the metabolome of E. glandulosa (Chapter 5), all treatments were handled as one experiment.

Chromatograms were uploaded to SpectConnect as five treatments groups: (1) E. glandulosa v self (stable temperature); (2) E. glandulosa v S. rugosum along vessels; (3) E. glandulosa v S. rugosum across vessels; (4) E. glandulosa v O. mucida along vessels; (5) E. glandulosa v O. mucida across vessels (3 replicates per treatment).

In all cases the elution threshold was set to $1 \mathrm{~min}$ and support threshold was set to $50 \%$ which was chosen as this was the most conservative level to align the tetracosane internal standard as a single row in the output matrices. Compound abundances (concentrations) were calculated using the integrated signal (IS) matrix produced by SpectConnect. The matrix row corresponding to the tetracosane internal standard was identified and the IS of all other compounds in each column normalised that of the relevant internal standard. Compound abundances were then divided by the weight of the relevant wood block chips to give compound abundance values $\mathrm{g}^{-1}$. Matrix rows were associated with their chromatogram peaks and putative compound identifications by cross-referencing the IS matrix with both the RT matrix produced by SpectConnect and the retention times of peaks in the chromatograms in which the compound appeared within AMDIS. Common contaminants of GC-MS datasets (e.g. disiloxanes which are hydrolysed from MSTFA+ $1 \%$ TMCS on contact with $\mathrm{H}_{2} \mathrm{O}$ ) were removed from the datasets along with chromatogram peaks that were found to comprise $<6$ mass spectra. All compounds (rows) in the final matrix (Appendix II) were numbered sequentially and unidentified compounds maintained these numbers as identifiers. Analytical compound names were cross referenced with the names of bioactive metabolites using GMD and other public repositories such as ChemSpider (Pence \& Williams 2010), PubChem (Wang et al. 2009) and the NIST Chemistry WebBook (Linstrom \& Mallard 2001) and analytes corresponding to the same metabolite were merged additively. 


\subsubsection{Statistical analysis of profiles of fungal metabolites from branch wood cores}

\subsubsection{Processing and normalisation of fungal metabolomes from woodblocks}

Data for each of the three experiments were uploaded to MetaboAnalyst 4.0: (1) effect of temperature on O. mucida; (2) effect of temperature on E. glandulosa; and (3) effect of replacement interactions on $E$. glandulosa. Data were uploaded as a sample $\mathrm{x}$ compound matrix using an un-paired design. Data filtering was not applied as the previous pre-processing and alignment steps had adequately removed variables likely to be non-informative. Data were normalised by median, g-log transformed and mean centred so that the dataset followed a near-normal distribution.

\subsubsection{Differential metabolomics of fungal metabolomes following stable and variable temperature treatment}

All analyses were undertaken in MetaboAnalyst 4.0. PLS-DA was used to visualise whether the relative abundances of any compounds in the dataset were likely to result in the separation of samples into the two experimental groups within acceptable confidence limits (95\%). Validation of the predictive accuracy of the model was calculated by cross-validation and inspection of Q2 values. Q2 values approaching 1 were taken as an indication of strong predictive accuracy and negative values as an indication of over-fitted models, which would lead to a high probability that any separation of samples seen in the two-dimensional PLS-DA scores plot arose by chance. Compounds that contributed most, due to the relative concentrations in the two experimental groups, to the separation of samples into experimental treatment groups were identified by VIP analysis. All compounds with VIP > 1.0 were deemed 'important' compounds and therefore to be compounds that were likely to differ in concentration between the two treatment conditions.

T-tests with a 5\% Benjamini-Hochberg FDR correction for multiple comparisons (Benjamini \& Hochberg 1995) were applied to test the null hypothesis of no significant differences in compound concentration between experimental conditions. Results of T-tests were combined with fold-change analysis using volcano plots (fold change threshold 2.0; raw P-value threshold 0.05) to visualise whether metabolites tended to be expressed with increased or decreased concentrations under variable compared with stable temperature.

\subsubsection{Differential metabolomics of Exidia glandulosa metabolome following replacement interactions along and across wood xylem vessels}

All analyses were undertaken in MetaboAnalyst 4.0. PLS-DA was used to visualise whether there was any evidence to suggest lasting effects were exerted by different initial colonising species on the $E$. glandulosa metabolome by dividing the experiment into three, rather than five, groups: (1) E. glandulosa (3 reps); O. mucida (6 reps); and S. rugosum (6 reps) within acceptable confidence limits (95\%). Validation of the predictive accuracy of the model was calculated by cross-validation and inspection of Q2 values. Q2 values approaching 1 were taken as an indication of strong predictive accuracy and negative values Page | 34 
as an indication of over-fitted models, which would lead to a high probability that any separation of samples seen in the two-dimensional PLS-DA scores plot arose by chance. Compounds that contributed most, due to the relative concentrations in the three experimental groups, to the separation of samples into experimental treatment groups were identified by variable importance in projection (VIP) analysis. All compounds with VIP > 1.0 were deemed 'important' compounds and therefore to be compounds that were likely to differ in concentration between the three treatment conditions.

Subsequently, to further explore the specific lasting effects of the identity of the initial colonising species on the metabolome of E. glandulosa, samples were divided into two separate experiments: (1) E. glandulosa vs self and all replacement treatments taking place along the xylem vessels; and (2) E. glandulosa vs self and all replacement treatments taking place across the xylem vessels. PLS-DA, VIP analysis and one-way ANOVA with a 5\% Benjamini-Hochberg FDR (Benjamini \& Hochberg 1995) followed by Fisher's LSD post-hoc tests were applied to each of the two experiments separately.

Finally, PLS-DA, VIP analysis and two-way analysis of variance (ANOVA) with a 5\% BenjaminiHochberg FDR correction for multiple comparisons (Benjamini \& Hochberg 1995) was applied to explore effects of all treatments and their interactions on the metabolome of E. glandulosa. Data were uploaded as a sample $x$ compound matrix using a two-factor independent samples design.

\subsection{Profiling the proteome of fungi in beech woodblocks}

\subsubsection{Extraction offungal metabolites from beech woodblocks}

All work was undertaken by Maynooth University, Ireland. The top third of woodblocks from each treatment group (one per species per treatment group; Section 2.5.2) were manually chipped. Weight of wood was standardised to the lowest weight between samples. As such $0.87 \mathrm{~g}$ of material was weighed for each sample and added to $50 \mathrm{ml}$ of cold phosphate buffer (50 mM potassium phosphate $\mathrm{pH} 7.5,1$ $\mu \mathrm{g} / \mathrm{ml}$ Pepstatin A, $1 \mathrm{mM}$ phenylmethylsulfonyl fluoride, 1mM EDTA) and agitated for $40 \mathrm{hrs}$ on a daisywheel $\left(4^{\circ} \mathrm{C}\right)$. The sample suspension was filtered and filtrate was spun at $25,000 \times \mathrm{g}$ for $30 \mathrm{~min}\left(4^{\circ} \mathrm{C}\right.$ ). The supernatant was brought to $15 \%(\mathrm{v} / \mathrm{v})$ trichloroacetic acid (TCA) and agitated on a daisywheel overnight $\left(4^{\circ} \mathrm{C}\right)$. Protein precipitate was spun at $1700 \times \mathrm{g}$ for $45 \mathrm{~min}\left(4^{\circ} \mathrm{C}\right)$ and pellets were washed with tris-buffered acetone $(20 \%(\mathrm{v} / \mathrm{v}) 50 \mathrm{mM}$ tris-base and acetone) with two additional tris-buffered acetone washes and one additional acetone wash. Dried-protein pellets were resuspended in $6 \mathrm{M}$ urea, $2 \mathrm{M}$ thiourea and $100 \mathrm{mM}$ Tris- $\mathrm{HCl} \mathrm{pH}$ 8.0. Protein concentrations were normalized following protein quantification by Bradford assay. Urea concentration was adjusted to $1 \mathrm{M}$ by addition of $50 \mathrm{mM}$ ammonium bicarbonate. Proteins were reduced by addition of $0.5 \mathrm{M}$ dithiothreitol and alkylated with 0.55 M IAA, with an incubation for $15 \mathrm{~min}$ at room temperature (in the dark; Collins 2013). Protein digestion was performed by using ProteaseMAX at a concentration of $0.01 \%(\mathrm{w} / \mathrm{v})$ followed by addition of sequencing-grade trypsin. Tryptic peptides were acidified with trifluoroacetic acid (TFA) and 
desalted using C18 Ziptips (Millipore ${ }^{\circledR}$ Ziptips C18; Dolan et al. 2014; O’Keeffe et al. 2014; Owens et al. 2015; Moloney et al. 2016).

\subsubsection{Direct shotgun LC-MS/MS analysis of peptide preparations from beech woodblocks}

All work was undertaken by Maynooth University, Ireland. $1 \mu \mathrm{g}$ of peptide mixtures were eluted onto a Q-Exactive (ThermoFisher Scientific, U.S.A) coupled to a Dionex RSLCnano for liquid chromatography (LC)-MS/MS analysis. LC gradients from 3-45\% B were run over $65 \mathrm{~min}$ and data were collected using a Top15 method for MS/MS scans. Spectra were analysed using the predicted protein databases of $E$. glandulosa HHB12029 (Nagy et al. 2016) and O. mucida CBS55879 (Barrasa 2014) using MaxQuant (version 1.6.2.3) with integrated Andromeda for database searching (Cox \& Mann 2008). MaxQuant parameters are as described in (Owens et al. 2015). Removal of possible contaminants, removal of protein hits found in less than two replicates $(\mathrm{n}=3)$, and Log2 transformation of LFQ intensities was performed using Perseus (version 1.4.1.3). Sequences of proteins detected in all samples across both experiments (Chapter 5 and Chapter 6) were exported as a single file in FASTA format (Appendix IV).

\subsubsection{Functional annotation of fungal proteomes}

All analyses were undertaken in Blast2GO Pro 5.2.4 (B2G; Conesa et al. 2005; Götz et al. 2008). All protein sequences were imported and subjected to the complete Gene Ontology (GO) pipeline. Sequences were aligned with both the NCBInr fungi database using BLASTp (BLAST expectation (E) value 1.0-3) and with the EMBL-EBI InterPro database (Jones et al. 2014). Results were mapped to the GO Association and Unitprot ID-Mapping service (GO annotation database version: 2018.09). NCBI $n r$ and InterPro results were merged and the Annotation module, which assigns GO terms above a user-defined cut off and in a non-redundant way (Götz et al. 2008), run with the following parameters: annotation cut-off 55; GO weight 5; BLAST E-value filter 1.0-6; HSP-Hit coverage cut-off 0; and hit filter 500. Annotated proteins were further subjected to GO Slim (Gene Ontology Consortium 2004) which is a reduction step that reduces GO annotations to a high-level view of the three ontologies thereby facilitating better comparison of GO terms between samples and studies and helps to reduce redundancy in functionally annotated datasets. Enzyme Commission (EC) codes were mapped to slimmed GO terms and loaded into KEGG pathway maps (Kanehisa \& Goto 2000). Data tables were exported as .txt files for further analysis in Excel.

Portions of the complete dataset were filtered to allow examination of functional annotations relevant to experiments and groups of treatments as required by filtering the dataset as needed in Microsoft Excel to produce gene lists which were used to select sequences in B2G. Slimmed functional annotations were explored by way of directed acyclic graphs (DAG) with default settings used to control the filtering of nodes during graph construction.

Page | 36 


\subsubsection{Differential analysis offungal proteomes}

\subsubsection{Enrichment analysis}

Using B2G enrichment analysis module, Fisher's exact tests with 5\% Benjamini-Hochberg FDR correction for multiple comparisons (Benjamini \& Hochberg 1995) were applied to pairwise treatment contrasts in both experiments to test the null hypothesis of no association between categories (Table 2.4). Test and reference sets were selected by re-importing gene lists generated using Excel to filter for proteins detected across all samples in each of the two experiments.

\subsubsection{Discrimination analysis and T-tests applied to proteomes of fungi under different temperature regimes}

All analyses were undertaken in MetaboAnalyst 4.0 by assigning unique protein identifiers to each protein using concatenation of protein identification and gene name in Excel. PLS-DA was used to visualise the proteomes of (1) O. mucida and (2) E. glandulosa under stable and variable temperature regimes within acceptable confidence limits (95\%). Validation of the predictive accuracy of the model was calculated by cross-validation and inspection of Q2 values. Q2 values approaching 1 were taken as an indication of strong predictive accuracy and negative values as an indication of over-fitted models, which would lead to a high probability that any separation of samples seen in the two-dimensional PLSDA scores plot arose by chance. Genes that contributed most, due to their relative concentrations, to the separation of samples into experimental treatment groups in the two experiments were identified by VIP analysis. All compounds with VIP > 1.0 were deemed 'important' compounds and therefore to be compounds that were likely to differ in concentration between treatment conditions.

T-tests with a 5\% Benjamini-Hochberg FDR correction for multiple comparisons (Benjamini \& Hochberg 1995) were applied to test the null hypothesis of no significant differences in protein concentration between temperature regimes. Results of T-tests were combined with fold-change analysis using volcano plots (fold change threshold 2.0; raw P-value threshold 0.05) to visualise whether proteins tended, in the main, to be expressed with increased or decreased concentrations under variable compared with stable temperature. 


\section{Chapter 3 - The role of environmental and spatial processes in structuring pioneer ligninolytic communities in beech branches}

\subsection{Introduction}

The decomposition of dead wood is effected by communities of ligninolytic fungi that develop within the wood and which therefore contribute fundamentally to the functioning of woodland ecosystems through the turnover of stored carbon and nutrients (Schwarze et al. 2000; Lonsdale et al. 2008; Pan et al. 2011; Purahong et al. 2018). Ligninolytic community development largely follows a successional course and different fungal species are typically noted to be associated with primary, early-secondary, latesecondary and tertiary successional stage. Community assembly trajectories will vary depending upon whether community development starts on the woodland floor or in the standing tree (Boddy 2001; Boddy et al. 2017). For the most part, assembly will begin long before the wood reaches the woodland floor as already well-decayed wood. Decay in roots, in the butts and tops of the standing trunk and of wounded sapwood by unspecialised pathogens are well-known examples of decay in standing trees.

The most common, yet largely unstudied, initial source of dead wood are whole dead branches attached to otherwise healthy, standing trees (Boddy 2001). Here the wood undergoes extensive decay, effected by the primary decay community which establishes from latent endophytic propagules (e.g. yeasts, spores and mycelial fragments) that were present asymptomatically in the functional wood. Later, once water content drops and the nutrient profile is more favourable, early-secondary colonists may begin to invade the community whilst wood is still attached to the standing tree (Boddy et al. 2017). In the earliest stages of community development, secondary invasion of attached dead wood is impeded by unfavourable abiotic conditions, including high water potential, poor gaseous regime, the remaining presence of antimicrobial secondary metabolites produced by the tree and low nutrient availability. While secondary and tertiary decay species are also likely to be present latently within functional sapwood, the pioneer community is simply better adapted to these stressful conditions (Boddy 2001; Boddy et al. 2017) and for these reasons attached dead branches have been described as a unique ecological niche (Stockland et al. 2012).

Dead attached branches constitute a major proportion of the dead wood in temperate woodland systems, particularly in older stands where the quantity of dead wood present as attached branches can be congruent with that of all other types of dead wood in the system (Svensson et al. 2014). While wounding may contribute to the reduced health of a branch, in these situations ligninolytic fungal communities are highly localised (Chapela \& Boddy 1988a, b; Rayner \& Boddy 1988; Boddy 2001). Whole tree branches, particularly the oldest lowest branches (Svensson et al. 2014), die as a result of 
stress factors such as light suppression as a result of crown growth or heavy competition from neighbouring trees (Hasenauer 1997; Boddy 2001; Hein \& Spiecker 2007) as part of a process known as natural pruning. While the endophytic and pioneer wood decay communities may include a number of weakly parasitic species (Boddy \& Rayner 1983a; Boddy 2001), their virulence is dependent upon a host already weakened by stress (Ragazzi et al. 2001). Once sapwood becomes dysfunctional, vegetative mycelia begins to grow out from those endophytic propagules capable of withstanding the chemical and environmental conditions within the newly dysfunctional wood and primary resource capture may be completed very quickly (Parfitt et al. 2010). In oak (Quercus robur and Q. petraea), ash (Fraxinus excelsior), hazel (Corylus avellana) and birch (Betula spp.) it has been shown that branches are rapidly colonised by relatively species poor communities developing extensive longitudinal colonies (Boddy \& Rayner 1983a; Boddy 2001). This rapid colonisation is thought to be effected by the extensive but sparse distribution of propagules throughout functional wood, followed by the fusing of compatible genotypes during the primary resource capture process. In beech (Fagus sylvatica), the pioneer community is known to be more complex due to the intricate patterning of interaction zone lines within the wood and is less well characterised (Boddy 2001; Boddy et al. 2017 and papers reviewed within).

In most ecosystems, with a few notable exceptions (Polis \& Hurd 1995; Mittelbach et al. 2001; Blakely \& Didham 2010), species richness holds a positive relationship with habitat area. This species-area relationship (SAR) is one of the most well documented and universally accepted ecological phenomena and has been recorded in ligninolytic fungal communities associated with CWD based on sporocarp surveys which considered habitat area in terms of the volume (Bader et al. 1995; Renvall 1995; Lindblad 1998) or surface area (Kruys \& Jonsson 1999; Heilmann-Clausen \& Christensen 2004) of woody debris units. There is debate however on the ability of sporocarp surveys to reflect the true structure of ligninolytic communities (Boddy 2001; Straatsma et al. 2001). Isolation of mycelia from decaying wood is recommended as the only way to identify the active ligninolytic fungi present in the substrate with certainty (Boddy 2001), but the presence of a SAR for mycelial individuals has never been investigated in dead wood. Previous studies of primary decay communities in attached branches have isolated and counted somatically compatible mycelial individuals (Boddy 2001 and papers reviewed within). These studies provided informative check lists of the organisms associated with attached dead branches and developed an understanding of how fungal communities start to form in these vital and unique early stages. However there have been no attempts to examine whether a SAR exists within attached dead branches or to link fungal community structure with the properties of their abiotic environment which is a fundamental goal in ecology. To facilitate this, isolation techniques may be supported by PCR and DNA sequencing methods likely to target the major source of fungal biomass within a sample of wood. Studies in fallen logs frequently employ next generation DNA sequencing (Ovaskainen et al. 2010; Kubartová et al. 2012; Ottosson et al. 2014; Hoppe et al. 2016). This highly sensitive approach would be cost-ineffective to apply in a way that might represent the diversity of an attached-branch community 
which organises itself in large, long decay columns within branches. It could also arguably produce a confusing picture from the DNA of both the active ligninolytic fungi, minor community members inhabiting interaction zones lines and other inter-mycelial spaces, and any remains of the endophytic community which has been shown to be dominated by general fungal taxa (Boddy et al. 2017). Alternatively DNA or RNA fingerprinting techniques may be used to rapidly provide a general indication of fungal OTU richness but from which community structure per se cannot be inferred (Rajala et al. 2011, 2012). As such, Sanger sequencing (Section 2.2.7) was considered here to be the most suitable approach to support isolation techniques.

Diversity indices such as those for species richness and diversity reduce the structural complexity of ecological communities down to a single value. As such, community structure is arguably better represented by a highly multivariate data 'cloud' comprising the identities and relative abundances of all species within and between samples. The structure of ligninolytic communities continually changes in time and space (Boddy 2000, 2001; Rajala et al. 2011; Ottosson et al. 2014; Hiscox et al. 2015a, 2016b). These dynamics are context dependent and are better studied from the perspective of CWD communities within which the pioneer community contributes minimally to decay processes and is rapidly replaced by the secondary succession (Boddy 2001; Hiscox et al. 2015a, 2016b). Community structure in three dimensions has been illustrated and species richness recorded for communities associated with Q. robur, Q. petraea, F. excelsior, C. avellana and Betula spp. (papers reviewed within Boddy 2001; Boddy et al. 2017) attached branches and in F. sylvatica trunks with strip cankering (Hendry et al. 1998). Further work is required to understand the structure of primary decay communities that form within attached branches, particularly within beech, and the degree to which they are shaped by local environmental (branch physical properties) and spatial (geographic distances within and between branches) factors. To understand the mechanisms that generate and drive the diversity and structure of attached branch communities, an understanding of their biogeographic distribution patterns is necessary (Martiny et al. 2006) which in turn may enable prediction of the system's likely responses to environmental change (Zhang et al. 2018). Biogeographic patterns are driven by deterministic processes that operate at local and regional scales such as habitat quality, dispersal limitations, mass effects and historical factors (Vellend 2010; Hanson et al. 2012). The within-branch abundance and distribution of genetically compatible endophytes from which the primary decay community forms is likely to be at least partially stochastic (Rodriguez et al. 2009) meaning that the abundance (colony size) and arrangement of mycelial individuals in the primary decay community might arise by chance rather than as a consequence of branch quality factors. Spatial variables can be used to account for variables that are not well understood and impossible to trace back to the pattern of endophytes from which the community developed: dispersal, spatially structured environmental variables and historical contingencies (Beisner et al. 2006; Peres-Neto \& Legendre 2010; Zhang et al. 2018). 
Furthermore, pure spatial effects and environmental gradients are components of every habitat but independently may give rise to similarly structured communities. Where pure spatial factors play a dominant role, communities are known to be spatially autocorrelated due to dispersal between adjacent habitat patches; when environmental conditions, which themselves exhibit spatial autocorrelation due to gradients and patchiness in energy inputs, exert strong effects, environmental distance results in decreasing similarity of community structure (Legendre 1993; Hanson et al. 2012). Environmental distance increases as habitat patches become less similar, regardless of their distribution in space. Variation partitioning is a powerful technique, capable of separating out these confounding effects of spatial autocorrelation and deterministic environmental factors on community composition (Borcard et al. 1992; Legendre 2008; Smith \& Lundholm 2010).

In the present study, detailed surveying of fungi associated with 16 dead attached F. sylvatica branches was undertaken at a minimally managed woodland site in South Wales. This study was facilitated by the application of RSS to the visible decay regions created by fungi in the non-functional sapwood. In combination with isolation methods and Sanger sequencing supported by PCR amplification of fungal DNA, these methods were considered to most faithfully represent the actively growing ligninolytic fungi within the substrata whilst allowing, for the first time ever, a quantification of the relative size of ligninolytic fungal colonies in nature. Given that the distribution of the propagules of these colonies is likely driven by neutral processes but that development of the mycelial community is subject to considerable environmental filtering, this work aimed to address whether mycelial community structure in dead attached branches likely arises because of spatial auto-correlation or more deterministic processes. To address this aim, the work asked two specific questions: (1) does the species richness or diversity of communities associated with dead attached branches exhibit a relationship with habitat area, as has been reported in woody debris? (2) does observed variation in community structure display a stronger relationship with measurable environmental variables or with purely spatial variables? Given that the mycelial community develops from propagules whose distribution and abundance within dysfunctional branches are likely to be driven by stochastic processes, it was hypothesised that spatial factors would play a more significant role and that communities would display spatial auto-correlation with branch physical characteristics playing a lesser role. However, assuming that branches with greater volume will naturally contain more propagules it was hypothesised that a positive SAR would be detected with branch volume.

\subsection{Outline of methods}

\subsubsection{Surveying methods}

Dead attached branches were cut down from standing beech trees at a site in Swansea, UK (Section 2.1.1). Slices of wood were removed at random locations along the length of each branch $(z$ distance 
from cut end; Section 2.1.2; Fig. 2.2) to reveal the decay regions produced by genetically incompatible fungal colonies within the wood, demarcated by interactions zone lines (Fig. 2.4). A RSS strategy was applied to decay regions (strata) within each slice to build up a three-dimensional picture of the community within each branch (Section 2.1.6 and Appendix I). Isolations from each RSS sampling location were grown on $2 \%$ MEA to promote rapid growth and pure cultures were identified by morphological comparison with known cultures or Sanger sequencing of fungal tissue (Sections 2.2.1 and 2.2.2). Where needed, these data were supplemented with identifications produced by Sanger sequencing fungal DNA from sawdust samples (Sections 2.2 .3 - 2.2.6). Bioinformatics were undertaken in Geneious R9 (Section 2.2.7). Abundance data were based on the number of RSS sampling cells occupied by fungi of the same identity within samples (Section 2.2.8). As such, abundance values were a proximate measure of both colony size and the relative number of cellular individuals within each colony. Wood volume and mass loss were calculated at both branch and slice levels (Section 2.1.6).

\subsubsection{Data analyses}

Data analyses were undertaking in R Studio (R Studio Team 2016; R Core Team 2018) and PRIMER 7 (Anderson et al. 2008a; Clarke \& Gorley 2015). Spearman-rank correlations were calculated in R to determine whether branch communities exhibited a SAR between either species richness or Simpson's diversity (1-D) and the predictor variables branch volume or lateral area (Section 2.2.8). To explore the general patterns of community structure in branches undergoing low- moderate- and extensivedecomposition, the measured decay gradient (Section 2.1.7) was divided into thirds and the resemblance matrix of community structure at each level plotted using nMDS (Section 2.2.9.2). BEST analysis was applied to determine whether the observed variation in community structure could equally well be described by a reduced dataset. This approach identifies, therefore, the species contributing the most to clustering or separation of samples within the nMDS thereby helping to explain the plotted ordination.

To determine wither community structure within the branches surveyed likely arose purely due to spatial auto-correlation or also due to an effect of branch physical characteristics (environment), distLMs were built in PERMANOVA+ with variation partitioned into spatial and environmental components (Section 2.2.9.3). Under variation partitioning, if the environmental partition explains a significant proportion of the observed variation in community structure, particularly if it is fitted into the model first, any effect of the variables on community structure can be interpreted with greater confidence given that any conflation with purely spatial effects in the study has been quantified. As such, once distLMs with variation partitioning had been fitted, further models were built wherein all environmental and spatial variables were considered independently (Section 2.2.9.4). All distLM models were run at various taxonomic and functional levels: species, order, phylum, guild and trophic mode. 


\subsection{Results}

\subsubsection{Fungal diversity and community composition}

A total of 16 branches were surveyed ranging in volume from $0.001-0.136 \mathrm{~m}^{3}$ and having lost between $16.7 \%$ and $75 \%$ of their original dry mass (Table 3.1). From these branches, the spatial arrangement of 4,238 records of 80 species were mapped (Appendix I). The Basidiomycota displayed lower species richness $(n=31)$ than the Ascomycota $(n=48)$ although the Basidiomycota had higher relative abundance, formed bigger colonies and produced fewer singletons (Table 3.2). The Basidiomycota were largely represented by members of the orders Russulales $(n=995)$, Agaricales $(n=822)$ and Polyporales $(n=687)$. The Ascomycota were largely members of the orders Xylariales $(n=544)$, Hypocreales $(n=$ 343 ) and Helotiales $(n=272)$. The five most commonly recorded species were the Basidiomycota Stereum rugosum, Ceriporiopsis gilvescens, Oudemansiella mucida and Cylindrobasidium evolvens and the ascomycete Neonectria coccinea. These fungi dominated the records accounting for $53.1 \%$ of overall abundance (Appendix I).

A single species, Mortierella sp., belonged to the Zygomycota and was isolated from a small decay column in branch CLY14 which had undergone substantial decay (mass loss $=47.2 \%$ ) and had a very loose bark layer. While fungi belonging to the Mortierella genus are more commonly known as soil saprotrophs, some species are known to degrade plant hemicellulose (Varnaitè \& Raudonienè 2008) and so in the present study the fungus was considered to be present opportunistically but contributing to the overall decomposition of the substratum. Trichoderma spp. were the sole fungi detected in several decay columns in four branches (CLY09, CLY12, CLY15 and CLY19; $n=14$ ) and their role as either mycoparasites or opportunistic scavengers of hemicellulose (Hendry et al. 2002) could not be ascertained within the present study. As such records relating to both genera were included in the study. Fungi associated with two small decay columns across the study, one within branch CLY04 (Basidiomycota) and one within CLY07 (Ascomycota), could not be identified further than phylum level.

Attached branch communities at the lower (0 - 25\% mass loss; decay stage 2) end and in the middle of the decomposition gradient $(25.1 \%$ - 50\% mass loss; decay stage 1$)$ were more similar in structure than those at the lower extreme ( $0-25 \%$ mass loss; decay stage 2$)$, as illustrated by the tighter clustering of points associated with decay stages 1 and 2 by nMDS ordination plotting (Fig. 3.1). The variation present in the highly multivariate dataset ( 80 species) could have been described equally well by a reduced dataset comprising just 13 species: Biscogniauxia nummularia, Cylindrobasidium evolvens, Eutypa lata, Exidia glandulosa, Hypoxylon fragiformme, Nectria coccinea, Oudemansiella mucida, Polyporus tuberaster, Schizopora radula, Stereum hirsutum, Stereum rugosum, Trametes versicolor and Trichoderma sp. (BEST analysis; Appendix I). Assemblages in the middle of the decomposition gradient seemed to be influenced 


\begin{tabular}{ccccccccc}
\hline Branch & $\begin{array}{l}\text { Volume, } \\
\mathbf{m}^{3}\end{array}$ & $\begin{array}{l}\text { Lateral } \\
\text { Area, } \mathbf{~ m}^{2}\end{array}$ & $\begin{array}{l}\text { Average } \\
\text { Density, } \\
\mathbf{g ~ c m}^{-3}\end{array}$ & $\begin{array}{l}\text { Mass } \\
\text { Loss, } \%\end{array}$ & $\begin{array}{l}\text { Species } \\
\text { Richness }\end{array}$ & $\begin{array}{l}\text { Diversity, } \\
\text { 1-D }\end{array}$ & Latitude & Longitude \\
\hline CLY01 & 0.009 & 0.667 & 0.55 & 23.6 & 5 & 1.199688 & 51.6061 & 4.016729 \\
CLY02 & 0.009 & 0.686 & 0.22 & 69.4 & 14 & 1.775702 & 51.6164 & 4.013357 \\
CLY03 & 0.136 & 4.641 & 0.53 & 26.4 & 9 & 1.627039 & 51.61371 & 4.018887 \\
CLY04 & 0.021 & 1.052 & 0.30 & 58.3 & 11 & 1.741682 & 51.60776 & 4.014639 \\
CLY06 & 0.006 & 1.011 & 0.44 & 38.9 & 7 & 1.197476 & 51.61449 & 4.018711 \\
CLY07 & 0.077 & 2.104 & 0.23 & 68.1 & 21 & 1.988531 & 51.61436 & 4.018611 \\
CLY08 & 0.021 & 0.881 & 0.18 & 75 & 5 & 1.076063 & 51.61428 & 4.018062 \\
CLY09 & 0.022 & 0.920 & 0.35 & 51.4 & 7 & 1.485252 & 51.61386 & 4.019785 \\
CLY10 & 0.030 & 1.329 & 0.59 & 18.1 & 6 & 1.304873 & 51.61473 & 4.01852 \\
CLY11 & 0.019 & 1.278 & 0.60 & 16.7 & 6 & 0.812632 & 51.61473 & 4.017319 \\
CLY12 & 0.006 & 0.510 & 0.42 & 41.7 & 18 & 2.236747 & 51.61476 & 4.018703 \\
CLY13 & 0.062 & 1.691 & 0.53 & 26.4 & 2 & 0.513068 & 51.61362 & 4.019059 \\
CLY14 & 0.001 & 0.007 & 0.38 & 47.2 & 4 & 0.923738 & 51.61362 & 4.019059 \\
CLY15 & 0.010 & 0.600 & 0.35 & 51.4 & 9 & 1.284852 & 51.61447 & 4.018356 \\
CLY19 & 0.010 & 0.467 & 0.44 & 38.9 & 7 & 1.268385 & 51.61428 & 4.017727 \\
CLY21 & 0.021 & 0.884 & 0.36 & 50 & 7 & 1.343208 & 51.6138 & 4.018831 \\
\hline
\end{tabular}

Table 3.2 Descriptive statistics for fungal colonies identified as belonging to the phyla Ascomycota and Basidiomycota. Abundance values were generated by counting the number of stratified sampling cells occupied by each fungus in branch slices. Singletons ${ }^{\mathrm{W}}$ and singletons ${ }^{\mathrm{B}}$ indicate the number of singletons counted within and between branches respectively

\begin{tabular}{lccccc}
\hline Phylum & $\begin{array}{c}\text { Max. } \\
\text { Abundance }\end{array}$ & $\begin{array}{c}\text { Median } \\
\text { Abundance }\end{array}$ & Std. Dev & Singletons $^{W}$ & Singletons $^{\mathbf{B}}$ \\
\hline Ascomycota & 232 & 16.44 & 38.25 & 20 & 12 \\
Basidiomycota & 661 & 44.74 & 99.53 & 5 & 2 \\
\hline
\end{tabular}

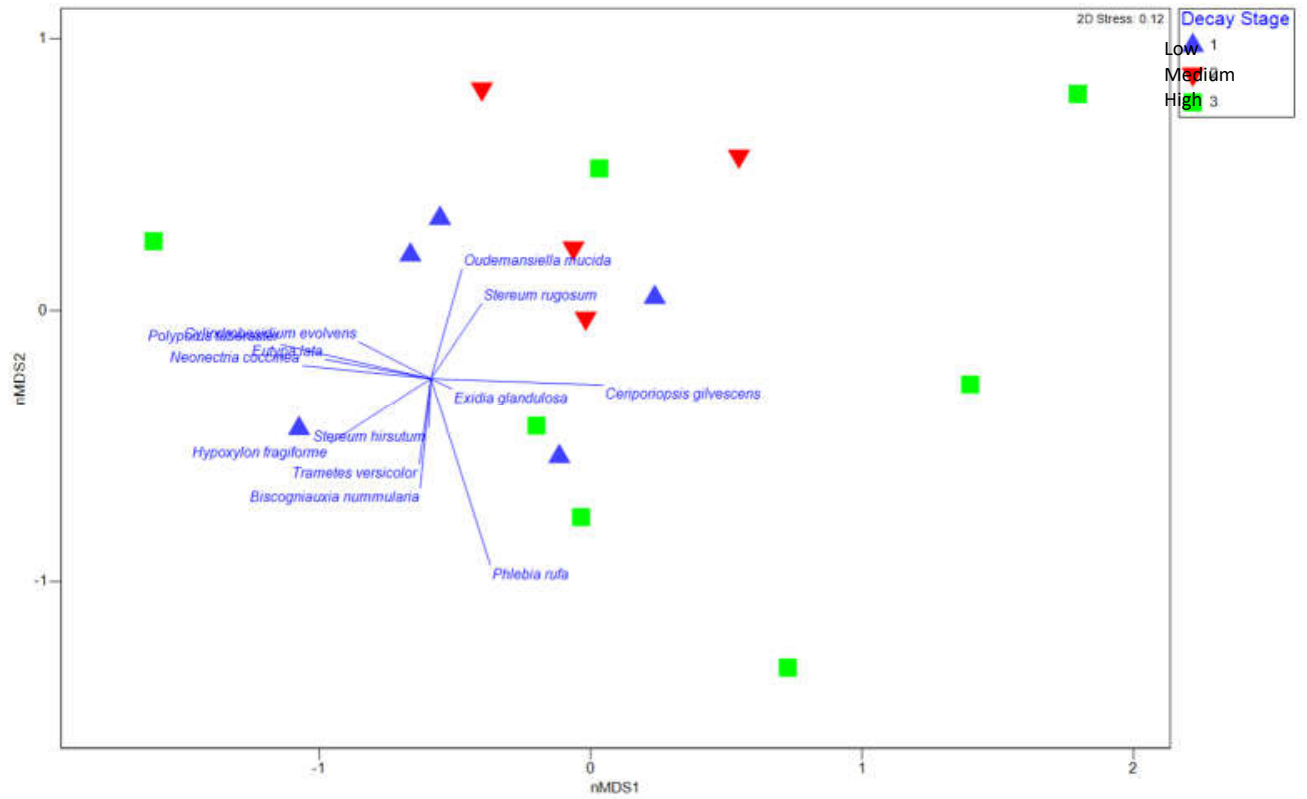

Figure 3.1 Unconstrained ordination of whole branch communities exhibiting low, medium and high levels of decomposition 
particularly by the presence of O. mucida and S. rugosum and the absence of S. hirsutum, H. fragiforme, T. versicolor and B. nummularia (Fig. 3.1).

Interesting patterns of community composition emerged when the relative abundance of the species identified above, along with species known from the literature to be important to the system, were plotted at different species and order levels over the mass loss gradient. At species level (Fig. 3.2), the weakly pathogenic and facultative saprotroph $N$. coccinea was associated with the greatest range of decomposition appearing in branches having lost between $16.7 \%$ and $58.3 \%$ of their dry mass and appearing in 7 out of the 13 branches in this range. Of the strict saprotrophs, O. mucida and S. hirsutum were affiliated with the greatest range of decomposition. They appeared in branches having lost between $18.1 \%$ and $51.4 \%$ of their dry mass and appeared in 9 and 8 out of 11 branches in this range respectively. Both O. mucida and S. hirsutum dominated in three assemblages in which they appeared. The xylariaceous species $H$. fragiforme also appeared over a wide range of decomposition and was associated with branches having lost between $17.7 \%$ and $50 \%$ of their dry mass, appearing in 4 out of 10 branches in this range, and occupied over $70 \%$ of the territory in the branch at the low extreme of the decomposition gradient. C. evolvens also dominated two of the earliest-stage branches having lost $18.1 \%$ and $23.6 \%$ of their dry mass but did not appear in any other assemblages. The two branches having undergone the most substantial decay, having lost $68.1 \%$ and $75 \%$ of their dry mass, were dominated by C. gilvescens. Inspection of the maps of these two assemblages showed that this species occupied multiple neighbouring decay zones. C. gilvescens also appeared as a small wedge-shaped colony on the perimeter of another assemblage, CLY15, which had undergone slightly less decay having lost $51.4 \%$ of its dry mass. T. versicolor appeared in three assemblages having lost between $41.7 \%$ and $51.4 \%$ of their dry mass and held decreasing proportions ofterritory over this range. The xylariaceous ascomycete $B$. nummularia dominated a single assemblage, CLY21, which had lost $50 \%$ of its dry mass loss and also appeared as small colonies in CLY11 and CLY06 which had lost 16.7\% and 38.9\% of their dry mass respectively. Five species appeared only once in the study: Pleurotus pulmonarius, Polyporus tuberaster, Calocera cornea, Nemania serpens and Hypholoma transiens. Of these P. tuberaster and C. cornea contributed significantly to the proportion of territory held in their assemblages and both branches had undergone extensive decomposition: $58.3 \%$ and $66.7 \%$ respectively.

At order level the Xylariales, Russulales and Agaricales dominated assemblages undergoing up to 51.4\% mass loss while in more decayed branches, the Polyporales became increasingly dominant (Fig. 3.3). In contrast, no discernible patterns emerged from community composition at species and order level plotted over the branch volume gradient and there were no discernible patterns of community composition at phylum level over either gradient (Appendix I). Most fungi identified were wood saprotrophs. Branches at all stages of decomposition and of all sizes were dominated by this guild and 
while some branches were more functionally diverse than others, shifts in functional complexity did not exhibit any discernible patterns over either gradient (Appendix I).

\subsubsection{Effect of branch volume on species richness and diversity}

Species richness within branches ranged between 2 and 21 species $(\bar{X}=8.62$, s.d. $=5.11)$ and species diversity (Simpson's 1-D) ranged between 0.51 and $2.24(\bar{X}=1.36$, s.d. $=0.44)$. No species area relationship was detected in the study. Spearman-rank correlation analysis found insignificant correlations between both species richness $(\rho=0.057 ; \mathrm{P}=0.835)$ and diversity $(\rho=0.157 ; \mathrm{P}=0.562)$ and branch volume. Lateral area also failed to correlate significantly with species richness $(\rho=0.083 ; P=$ $0.760)$ and diversity $(\rho=0.079 ; P=0.771)$. Furthermore, there was no significant correlation between either species richness $(\rho=0.446 ; P=0.084)$ or diversity $(\rho=0.427 ; P=0.099)$ and mass loss of the wood (Appendix I).

\subsubsection{Quantification of the unique effect of environmental factors on community composition through variation partitioning}

The environmental partition was more important in explaining variance in community structure at all taxonomic (species, order and phylum) and functional (trophic mode; guild) levels considered as revealed by variation partitioning and distLM analysis (Table 3.3; Appendix 1). In all cases, the environmental component was fitted first and explained a larger proportion of the explained variation with greater confidence than the spatial component. At species level 12.7\% of variation was explained by the environmental partition alone (pseudo-F $=6.4266, \mathrm{P}=0.001$, residual $d . f .=133$ ) while the spatial partition explained an additional $67.2 \%$ of the variation which was a significant portion of the remaining unexplained variation (pseudo- $\mathrm{F}=3.8671 ; \mathrm{P}=0.001$; residual $d . f .=130$ ). Thus, the environmental partition explained approximately twice as much variation as the spatial partition alone once the environmental component had been fitted. At order level, approximately 3.5 times as much variation was explained by fitting the environmental partition alone $(18.5 \%$ variation, pseudo- $\mathrm{F}=10.068, \mathrm{P}=0.001$, Residual d.f. $=$ $133)$ as that explained by the spatial partition once the environmental component had been fitted $(5.4 \%$ variation, pseudo- $\mathrm{F}=3.0825, \mathrm{P}=0.001$, Residual d.f. $=130$ ). At phylum level, the environmental partition alone was fitted to explain $21.5 \%$ of variation (pseudo- $\mathrm{F}=12.133 ; \mathrm{P}=0.001$; Residual d.f. $=133$ ).

There was an approximately eleven-fold difference in the variation in trophic mode explained by the reduced model fitting only the environmental partition (44.2\% variation, $p$ seudo-F $=35.176, \mathrm{P}=0.001$, residual $d . f .=133$ ) and the additional variation explained by subsequently fitting the spatial partition (4.6\% variation, pseudo- $\mathrm{F}=3.9382, \mathrm{P}=0.001$, residual d.f. $=130)$. Similarly, the environmental partition explained over ten times the variation in community structure by fungal guild ( $34.7 \%$ variation, $p$ seudo-F 


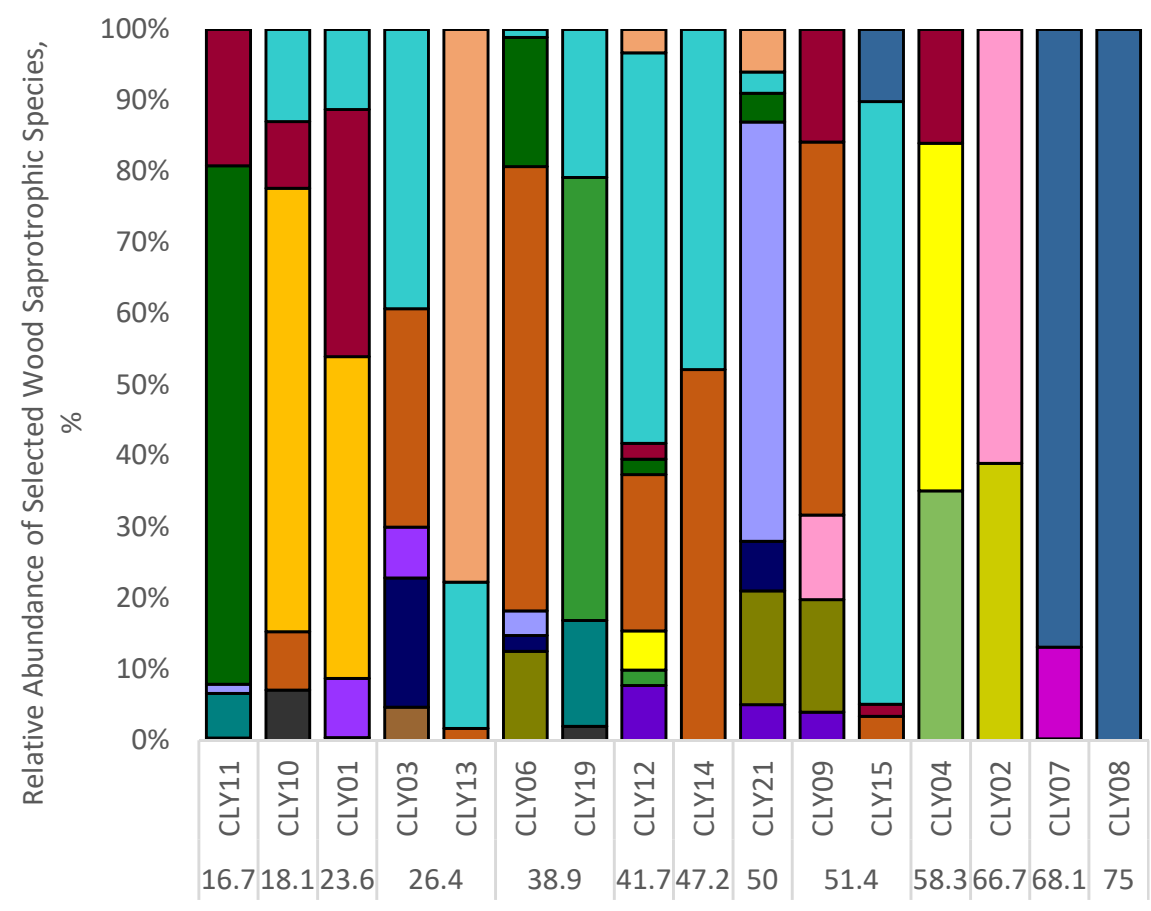

$\square$ Stereum rugosum

$\square$ Ceriporiopsis gilvescens

口Oudemansiella mucida

- Neonectria coccinea

$\square$ Cylindrobasidium evolvens

- Hypoxylon fragiforme

$\square$ Stereum hirsutum

$\square$ Schizopora radula

$\square$ Polyporus tuberaster

$\square$ Biscogniauxia nummularia

$\square$ Peniophora lycii

$\square$ Annulohypoxylon cohaerens

$\square$ Phlebia rufa

Exidia glandulosa

$\square$ Nemania serpens

$\square$ Pezicula cinnamomea

- Eutypa lata

$\square$ Calocera cornea

- Trametes versicolor

口Pleurotus pulmonarius

$\square$ Diatrype stigma

Branch Mass Loss, \%

Figure 3.2 Relative abundance of selected species over the decomposition gradient.

Species selected had either been identified by BEST analysis as being important in variation in community structure in surveyed attached branches or are known to be important ligninolytic communities from the literature (Boddy \& Rayner 1983a; Boddy 2001; Boddy \& Heilmann-Clausen 2008).

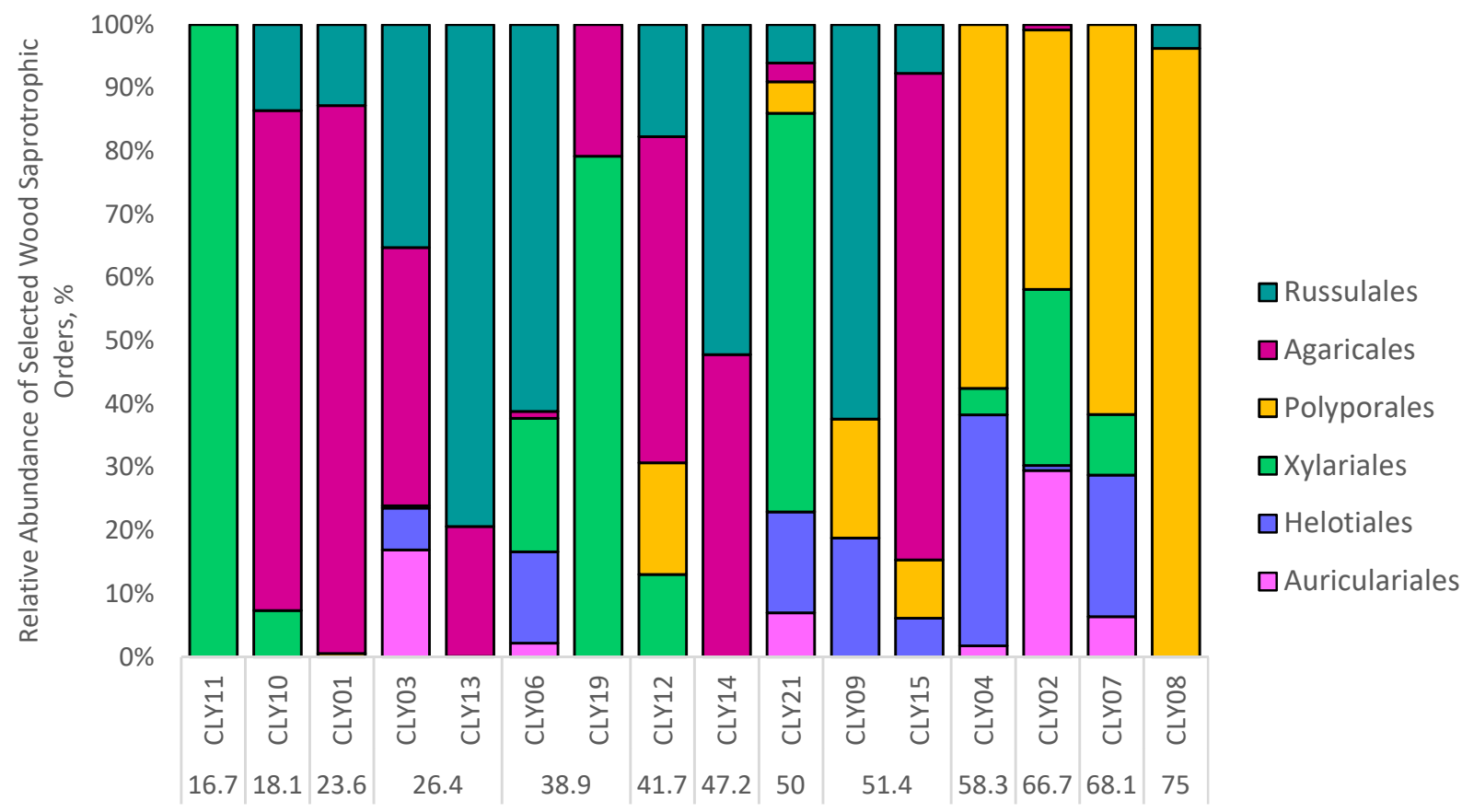

Branch Mass Loss, \%

Figure 3.3 Relative abundance of selected species at order level over the decomposition gradient.

Species selected had either been identified by BEST analysis as being important in variation in community structure in surveyed attached branches or are known to be important ligninolytic communities from the literature (Boddy \& Rayner 1983a; Boddy 2001; Boddy \& Heilmann-Clausen 2008). 
$=23.526, \mathrm{P}=0.001$, residual $d . f .=133)$ as did the spatial partition $(3.2 \%$ variation, $p$ seudo- $\mathrm{F}=2.2297, \mathrm{P}=$ 0.017 , residual d.f. $=130)$.

\subsubsection{Effect of branch physical characteristics and spatial variables on community structure}

While variation partitioning had revealed that the environmental partition was more important than the spatial partition in driving differences in community structure at various taxonomic and functional levels (Section 3.2.3), full distLM models were significant in all cases except at phylum level. As such, the influence of individual environmental and spatial variables on community structure were further examined by building distLM models without variation partitioning (Table 3.4; Appendix I). Mass loss

Table 3.3 Variation partitioning by distance-based linear modelling highlighting the effect of the spatial and environmental partitioned predictor variables on variance in community structure.

Selection criterion: adjusted R2; selection procedure: step-wise (distLM, level of taxonomy or functional aggregation the model was built at; Predict. Comp., predictor component; ss, sum of squares; F, F statistic; P-value, probability value; Sig, significance level; ${ }^{* * *} \mathrm{P} \leq 0.001 ;{ }^{* *} \mathrm{P} \leq 0.01 ;{ }^{*} \mathrm{P} \leq 0.05$; n.s., not significant; Expl. Var, \% of variance explained by the explanatory variable; Cum. Expl. Var, \% cumulative variation explained by the model following step-wise fitting of variables; Res.df, degrees of freedom of the residuals). Left aligned predictor variables show the results of marginal tests; indented predictor variables marked + show the results of sequential tests.

\begin{tabular}{|c|c|c|c|c|c|c|c|c|c|}
\hline $\operatorname{distLM}$ & Predict. Comp & SS & F & $\begin{array}{c}\text { P- } \\
\text { value }\end{array}$ & Sig. & $\begin{array}{c}\text { Expl. } \\
\text { Var, \% }\end{array}$ & $\begin{array}{c}\text { Cum. Expl. } \\
\text { Var, \% }\end{array}$ & Adj $R^{2}$ & Res.df \\
\hline \multirow[t]{4}{*}{ Species } & Spatial & 54537 & 4.3053 & 0.001 & $* *$ & 8.9 & & & 133 \\
\hline & Environmental & 78007 & 6.4266 & 0.001 & $* *$ & 12.7 & & & 133 \\
\hline & +Env & 78007 & 6.4266 & 0.001 & $* *$ & 12.7 & 12.7 & 0.10691 & 133 \\
\hline & +Spatial & 44088 & 3.8671 & 0.001 & $* *$ & 7.2 & 19.8 & 0.16116 & 130 \\
\hline \multirow[t]{4}{*}{ Order } & Spatial & 56101 & 5.4032 & 0.001 & $* *$ & 10.9 & & & 133 \\
\hline & Environmental & 95572 & 10.068 & 0.001 & $* *$ & 18.5 & & & 133 \\
\hline & + Env & 95572 & 10.068 & 0.001 & $* *$ & 18.5 & 18.5 & 0.16669 & 133 \\
\hline & +Spatial & 27948 & 3.0825 & 0.001 & $* *$ & 5.4 & 23.9 & 0.20408 & 130 \\
\hline \multirow[t]{3}{*}{ Phylum } & Spatial & 9401.9 & 1.6445 & 0.12 & $* *$ & 3.6 & & & 133 \\
\hline & Environmental & 56481 & 12.133 & 0.001 & $* *$ & 21.5 & & & 133 \\
\hline & + Env & 56481 & 12.133 & 0.001 & $* *$ & 21.5 & 21.5 & 0.19716 & 133 \\
\hline \multirow[t]{4}{*}{ Trophic } & Spatial & 9384.2 & 3.8687 & 0.001 & $* *$ & 8.0 & & & 133 \\
\hline & Environmental & 51728 & 35.176 & 0.001 & $* *$ & 44.2 & & & 133 \\
\hline & + Env & 51728 & 35.176 & 0.001 & $* *$ & 44.2 & 44.2 & 0.42984 & 133 \\
\hline & +Spatial & 5431.3 & 3.9382 & 0.001 & $* *$ & 4.6 & 48.8 & 0.46528 & 130 \\
\hline \multirow[t]{4}{*}{ Guild } & Spatial & 11185 & 3.1378 & 0.001 & $* *$ & 6.6 & & & 133 \\
\hline & Environmental & 58662 & 23.526 & 0.001 & $* *$ & 34.7 & & & 133 \\
\hline & + Env & 58662 & 23.526 & 0.001 & $* *$ & 34.7 & 34.7 & 0.33195 & 133 \\
\hline & +Spatial & 5409.7 & 2.2297 & 0.017 & * & 3.2 & 37.9 & 0.34998 & 130 \\
\hline
\end{tabular}


Table 3.4 Distance-based linear modelling highlighting the effect of individual branch physical characteristics and spatial variables on variation in community structure.

Selection criterion: adjusted R2; selection procedure: step-wise (distLM, level of taxonomy or functional aggregation the model was built at; Predict. Comp., predictor component; ss, sum of squares; F, F statistic; P-value, probability value; Sig, significance level; ${ }^{* *} \mathrm{P} \leq 0.001$; ${ }^{* *} \mathrm{P} \leq 0.01 ; * \mathrm{P} \leq 0.05$; n.s., not significant; Expl. Var, \% of variance explained by the explanatory variable; Cum. Expl. Var, \% cumulative variation explained by the model following step-wise fitting of variables; Res.df, degrees of freedom of the residuals). Left aligned predictor variables show the results of marginal tests; indented predictor variables marked + show the results of sequential tests.

\begin{tabular}{|c|c|c|c|c|c|c|c|c|c|}
\hline distLM & Predict. Var. & SS & F & $\begin{array}{c}\text { P- } \\
\text { value }\end{array}$ & Sig. & $\begin{array}{c}\text { Expl. } \\
\text { Var., \% }\end{array}$ & $\begin{array}{c}\text { Cum. } \\
\text { Expl. Var, } \\
\%\end{array}$ & $\operatorname{Adj} R^{2}$ & Res.df \\
\hline \multirow[t]{12}{*}{ Species } & Z & 16241 & 3.655 & 0.001 & * & 2.6 & & & \\
\hline & Diameter & 24454 & 5.5795 & 0.001 & $* *$ & 4.0 & & & \\
\hline & Mass Loss & 36791 & 8.5732 & 0.001 & $* *$ & 6.0 & & & \\
\hline & Tree Condition & 23479 & 5.3482 & 0.001 & $* *$ & 3.8 & & & \\
\hline & Latitude & 18741 & 4.2352 & 0.001 & $* *$ & 3.0 & & & \\
\hline & Longitude & 25558 & 5.8424 & 0.001 & $* *$ & 4.1 & & & 135 \\
\hline & +Mass Loss & 36791 & 8.5732 & 0.001 & $* *$ & 6.0 & 6.0 & 0.052748 & 135 \\
\hline & +Longitude & 24282 & 5.862 & 0.001 & $* *$ & 3.9 & 9.9 & 0.085678 & 134 \\
\hline & +Diameter & 21097 & 5.255 & 0.001 & $* *$ & 3.4 & 13.3 & 0.11382 & 133 \\
\hline & +Latitude & 18257 & 4.6731 & 0.001 & $* *$ & 3.0 & 16.3 & 0.13763 & 132 \\
\hline & +Tree Status & 11473 & 2.9806 & 0.001 & * & 1.9 & 18.2 & 0.15038 & 131 \\
\hline & $+Z$ & 10195 & 2.6828 & 0.004 & * & 1.7 & 19.8 & 0.16116 & 130 \\
\hline \multirow[t]{12}{*}{ Order } & Z & 8503.9 & 2.2603 & 0.041 & * & 1.6 & & & \\
\hline & Diameter & 23619 & 6.4704 & 0.001 & $* *$ & 4.6 & & & \\
\hline & Mass Loss & 46137 & 13.052 & 0.001 & $* *$ & 8.8 & & & \\
\hline & Tree Condition & 35435 & 9.946 & 0.001 & $* *$ & 6.9 & & & \\
\hline & Latitude & 20554 & 5.5959 & 0.001 & $* *$ & 4.0 & & & \\
\hline & Longitude & 24406 & 6.6968 & 0.001 & $* *$ & 4.7 & & & 135 \\
\hline & +Mass Loss & 48172 & 13.889 & 0.001 & $* *$ & 9.3 & 9.3 & 0.086566 & 135 \\
\hline & +Tree Status & 34403 & 10.626 & 0.001 & $* *$ & 6.7 & 16.0 & 0.14736 & 134 \\
\hline & +Diameter & 12997 & 4.1077 & 0.003 & * & 2.5 & 18.5 & 0.16669 & 133 \\
\hline & +Latitude & 12328 & 3.9834 & 0.002 & * & 2.4 & 21.0 & 0.18497 & 132 \\
\hline & +Longitude & 10312 & 3.3925 & 0.003 & * & 2.0 & 22.9 & 0.19948 & 131 \\
\hline & $+Z$ & 5308.6 & 1.7565 & 0.089 & n.s & 1.1 & 23.9 & 0.20408 & 130 \\
\hline \multirow[t]{9}{*}{ Phylum } & Z & 1352.6 & 0.69826 & 0.484 & n.s & 0.5 & & & \\
\hline & Diameter & 32456 & 19.016 & 0.001 & $* *$ & 12.3 & & & \\
\hline & Mass Loss & 16967 & 9.3147 & 0.001 & $* *$ & 6.6 & & & \\
\hline & Tree Condition & 15986 & 8.7416 & 0.002 & * & 6.1 & & & \\
\hline & Latitude & 3556.9 & 1.8518 & 0.155 & n.s & 1.4 & & & \\
\hline & Longitude & 1085.5 & 0.55977 & 0.577 & n.s & 0.4 & & & 135 \\
\hline & +Diameter & 32456 & 19.016 & 0.001 & $* *$ & 12.3 & 12.3 & 0.11698 & 135 \\
\hline & +Mass Loss & 16203 & 10.136 & 0.001 & $* *$ & 6.2 & 18.5 & 0.17295 & 134 \\
\hline & +Tree Status & 7822.4 & 5.0409 & 0.015 & * & 3.0 & 21.5 & 0.19716 & 133 \\
\hline
\end{tabular}




\begin{tabular}{|c|c|c|c|c|c|c|c|c|c|}
\hline distLM & Predict. Var. & SS & $\mathbf{F}$ & $\begin{array}{c}\text { P- } \\
\text { value }\end{array}$ & Sig. & $\begin{array}{c}\text { Expl. } \\
\text { Var., \% }\end{array}$ & $\begin{array}{c}\text { Cum. } \\
\text { Expl. Var, } \\
\%\end{array}$ & Adj $R^{2}$ & Res.df \\
\hline \multirow[t]{11}{*}{ Trophic } & $Z$ & 2399.4 & 2.8285 & 0.055 & n.s & 2.1 & & & \\
\hline & Diameter & 48842 & 96.851 & 0.001 & $* *$ & 41.8 & & & \\
\hline & Mass Loss & 2314.8 & 2.7267 & 0.048 & * & 2 & & & \\
\hline & Tree Condition & 3795.3 & 4.5291 & 0.01 & * & 3.2 & & & \\
\hline & Latitude & 5880.7 & 7.1495 & 0.001 & $* *$ & 5 & & & \\
\hline & Longitude & 812.7 & 0.94492 & 0.423 & n.s & 0.7 & & & 135 \\
\hline & +Diameter & 48842 & 96.851 & 0.001 & $* *$ & 41.8 & & 0.41342 & 135 \\
\hline & +Latitude & 4812.3 & 10.192 & 0.001 & ** & 4.1 & 45.9 & 0.45081 & 134 \\
\hline & +Mass Loss & 2338.2 & 5.104 & 0.003 & * & 2 & 47.9 & 0.46713 & 133 \\
\hline & +Tree Status & 575.95 & 1.2597 & 0.253 & n.s & 0.5 & 48.3 & 0.46817 & 132 \\
\hline & $+Z$ & 492.73 & 1.0783 & 0.317 & n.s & 0.4 & 48.8 & 0.46849 & 131 \\
\hline \multirow[t]{10}{*}{ Guild } & $\mathrm{Z}$ & 4049.1 & 3.3097 & 0.014 & * & 2.4 & & & \\
\hline & Diameter & 50449 & 57.348 & 0.001 & ** & 29.8 & & & \\
\hline & Mass Loss & 5928.4 & 4.9016 & 0.003 & * & 3.5 & & & \\
\hline & Tree Condition & 7602 & 6.3505 & 0.001 & $* *$ & 4.5 & & & \\
\hline & Latitude & 5071.4 & 4.1712 & 0.01 & * & 3 & & & \\
\hline & Longitude & 1686.6 & 1.3592 & 0.247 & n.s & 1 & & & 135 \\
\hline & +Diameter & 50449 & 57.348 & 0.001 & ** & 29.8 & & 0.29295 & 135 \\
\hline & +Mass Loss & 6078.7 & 7.2288 & 0.001 & $* *$ & 3.6 & 33.4 & 0.32413 & 134 \\
\hline & +Latitude & 3770.3 & 4.6043 & 0.01 & * & 2.2 & 35.6 & 0.34184 & 133 \\
\hline & +Tree Status & 2361.5 & 2.9256 & 0.044 & * & 1.4 & 37 & 0.35123 & 132 \\
\hline
\end{tabular}

was the most important variable driving variation in community structure at species level and all other variables also contributed to explaining significant portions of the residual variation. The full model explained $19.8 \%$ of the variation ( $p$ seudo- $\mathrm{F}=2.6828, \mathrm{P}=0.004$, residual $d . f .130$ ) and included all variables fitting mass loss first and $\mathrm{Z}$ distance last. Mass loss was again the most important driver of community structure variation at order level. A reduced model fitting all variables except $\mathrm{z}$ distance explained $22.9 \%$ of the observed variation ( $p$ seudo- $\mathrm{F}=3.3925 ; \mathrm{P}=0.003$; Residual d.f. $=131$ ). $\mathrm{Z}$ distance was also fitted into the full model but did not explain a significant portion of the residual variance.

When considering communities at phylum level and in terms of their functional complexity however, diameter became the most important driver of variation. At phylum level the full model, fitting diameter first, mass loss and whether the host tree was alive explained $21.5 \%$ of observed variation (pseudo-F $=$ 5.0409, $\mathrm{P}=0.015$, Residual d.f. $=133$ ). All spatial parameters were excluded from the model. $47.9 \%$ of variation in fungal trophic mode could be explained by a reduced model fitting diameter, latitude and mass loss (pseudo-F $=5.1040, \mathrm{P}=0.003$, residual d.f. $=133$ ). The full model also fitted tree status and $\mathrm{Z}$ distance but was insignificant ( $p$ seudo- $\mathrm{F}=1.0783, \mathrm{P}=0.317$, residual $d . f .=131$ ) with the two additional variables explaining only $0.5 \%$ and $0.4 \%$ of the variation respectively. When examined by fungal guild a full model fitting diameter, mass loss, latitude and tree status explained $37 \%$ of the variation in community structure (pseudo- $\mathrm{F}=2.956, \mathrm{P}=0.044$, residual $d . f .=132$ ).

Page | 50 


\subsection{Discussion}

Dead attached branches were structured by the physical characteristics of the branches they inhabited, with spatial autocorrelation playing a lesser role. At all taxonomic and functional levels studied, mass loss, wood diameter and whether the tree was dead or alive were major drivers of observed variance in structure. This was not simply due to slices being closer to each other geographically, either within the site sampling space (highly similar latitude and longitude values) or within branch sampling spaces (highly related $z$ values). While these spatial variables did explain a significant portion of the residual variation in community structure, the physical similarity of slices between branches was always more important in explaining variation in structure. This was true at all taxonomic and functional levels considered, with environment accounting for at least twice and up to 11 times more of the variation in community structure than the spatial partition. The sole exception to this was that at phylum level, at which spatial factors had no bearing at all on variation in community structure.

These results provided confidence in interpreting the effect of individual explanatory variables on variation in community structure without conflating true niche separation with spatial autocorrelation. The most important of these at species and order level was mass loss of the wood while at phylum level and both functional levels, wood diameter played the most significant role. It was not possible to avoid pseudo-replication sensu Hurlbert (1984) in the study while also addressing the interesting question of whether fungi are simply spatially aggregated within branches or whether their configuration, i.e. the space they colonise within branches, is determined by the physical characteristics of the surrounding wood. Given that colonies present as longitudinal decay columns, cellular individuals of the same fungus clearly display spatial aggregation in closely-related regions of branches in the $z$ direction, although occasionally multiple disconnected decay regions of fungi of the same species identity occur in the same branch. The question of interest is whether this configuration arises purely by chance, due to the historical arrangement of propagules, or because cellular individuals of the colony associate themselves with certain physical parameters of their environment. Mass loss is a fungal-driven process but also relates to the quality of the remaining lignocellulose resource in terms of its nutrient profile and drying regime, with more degraded wood likely being subject to far less stable moisture levels. Diameter is also related to microhabitat availability, as a greater diameter of wood likely buffers against excessive drying and fluctuations in temperature. The variance of mass loss and diameter values within a single branch, measured here in terms of the mass loss and diameter of individual slices, is representative of the range of microhabitats available within that branch. Whether cellular individuals, i.e. parts of a colony, associate with those different microhabitats is of interest. Wood slices within branches with similar mass loss values will have been available for pioneer colonists for more or less the same length of time however, so they are not fully independent of each other. As such, fully randomised methods were applied at all stages of sampling to minimise the effects of pseudo-replications as much as possible. 
It is interesting, given that fungal cells will be spatially aggregated as decay columns in the $z$ direction, that $z$ value which was treated as a purely spatial variable, did not contribute significantly to many of the unpartitioned models. The variable was fitted as an explanator of significant residual variation only when considering the structure of communities classified by species, phylum and trophic mode, was always the last or penultimate variable to be fitted and explained an insignificant degree of residual variation in the structure of phyla and trophic mode. This should alleviate some concern over the impact of pseudo-replication in the study, although the results reported should be considered to be representative only of the branches sampled in the present study. Future studies, employing a similar design in different geographical areas, would provide replication (Oksanen 2003).

The present study set out to determine whether a SAR exists in communities of ligninolytic fungi associated with attached branches of F. sylvatica and the extent to which branch quality and spatial factors played roles in driving variation in their structure. Notably, no SAR, either positive or negative, was detected in the study. This is inconsistent with the findings of many other studies, but might be indicative that habitat heterogeneity within branches does not necessarily increase with increasing branch volume (Báldi 2008). In the case of decaying tree branches, greater variety of microhabitats come in the form of different levels of decomposition at different points along their length and a steeper moisture gradient between the outer and inner wood (Stockland et al. 2012), factors which are driven principally by advancing decay rather than branch size. However, no correlations were found between mass loss and either species richness or diversity. Within attached branches, species richness is a function not only of habitat size but also of decay column size which in turn is a function of the historical spatial aggregation of genetically compatible endophytes within the wood at time of death. Larger colony size drastically alters the ability of less competitive ligninolytic fungi to defend territory even against highly aggressive antagonists displaying a patchier distribution (O'Leary et al. 2018). Primary decay fungi that have been able to produce larger colonies from the outset, a process with high stochasticity, may be able to maintain those large colony sizes over the long term. For these reasons, chance may play a bigger role than deterministic processes such as branch physical characteristics in determining community richness and evenness over spatial and temporal scales in dead attached branches, which would explain the absence of SAR in the present study.

The sampling approaches applied in the present study provide, for the first time, a measure of relative abundance of mycelial individuals within a naturally-formed wood decay system. The study demonstrated the high species richness of communities affiliated with dead attached F. sylvatica branches compared with those of other angiosperm trees in temperate woodlands. A total of 80 species were identified from 16 branches with total volume of $0.46 \mathrm{~m}^{3}$. Previously, 12 species of Basidiomycota had been associated with Q. robur from a study of 16 dead attached branches (Boddy \& Rayner 1983a) while in F. excelsior a total of 12 species ( 3 of Ascomycota, 7 of Basidiomycota with a further 2 
ascomycete species unidentified) characterised 18 branches. These previous works could not be supported by DNA sequencing techniques and so the present study has greater sampling depth however the pattern of decay regions that present within other angiospermous wood are considerably less complex. As such, the comparisons made here are informative. Total branch volumes were not stated in previous work and so cannot be compared. Extensive decomposition of the wood substratum was observed in the present study, with attached branches having lost up to $75 \%$ of their original dry mass and those having lost up to $66.7 \%$ of their dry mass being dominated by species known to be primary decay fungi (Boddy 2001 and papers reviewed within; Heilmann-Clausen \& Boddy 2008). These were O. mucida (10 branches), H. fragiforme (4 branches), S. rugosum (3 branches), C. evolvens (3 branches), E. glandulosa (3 branches), E. lata (2 branches), and Sz. radula (2 branches). Of these O. mucida was perhaps the most important, being affiliated with the greatest range of decomposition $(18.1 \%-51.4 \%$ mass loss) and with a greater number of branches overall (10 out of 16 branches) than any other species in the study. This was likely a consequence of O. mucida's highly defensive strategy (Anke 1995; Bartlett et al. 2002). The early-secondary successional species S. hirsutum and T. versicolor (Boddy 2000) were also commonly identified ( 8 and 3 branches respectively) and determined to contribute significantly to variation between communities. Of these, S. hirsutum was affiliated with a surprisingly wide range of decomposition, equal to that of O. mucida. Both O. mucida and S. hirsutum were present with low relative abundance compared with the dominant C. evolvens in branch CLY10 which had only lost $18.1 \%$ of its dry mass. The species did not dominate in 5 out of the 8 branches with which it was affiliated. Taken together these results suggest that the S. hirsutum strains detected may have been present as and colonised from latent endophytes and may be behaving as a primary decay fungus in the present study. Conversely the early-secondary species T. versicolor appeared only in branches having lost between $41.7 \%$ and $51.4 \%$ dry mass and only as small colonies on the perimeter of slices indicating that this species may have arrived in the form of air-borne propagules. T. versicolor was a minor constituent of all three branches with which it was affiliated and was not detected in any of the five most decayed branches indicating that this normally highly combative species (Hiscox et al. 2016a) was unable to destabilise these well-established communities. This is likely to be a product of the complexity of interactions taking place in three dimensional space in decaying branches (Toledo et al. 2016) and of the home advantage afforded to the primary decay residents in terms of the size of their well-established colonies (O'Leary et al. 2018).

The weakly pathogenic and facultative saprotroph N. coccinea (7 branches) was affiliated with a greater range of decomposition than $O$. mucida and S. hirsutum however this species commonly shared space with, and was isolated from the same wood chips as, other fungi known to be important ligninolytic fungi (Appendix I) and so while N. coccinea was present in substantially decomposed branches (up to $58.3 \%$ mass loss), the species is unlikely to be a major driver of those levels of decay. The most decayed branches, CLY07 and CLY08 which had lost 68.1 and 75\% of their mass respectively, were both 
dominated by a single species C. gilvescens. The successional status of this species is unknown however its colonies transcended colony boundary lines in the wood which were likely to be relic lines from previous colonisers. Some species, such as $\mathrm{O}$. mucida, produce pseudosclerotal plates which can give the appearance of colony boundary lines within wood. These are also produced in pure culture on artificial media however, a characteristic that $C$. gilvescens did not exhibit. As such the domination of $C$. gilvescens in these well-decomposed branches is likely to have been secured through replacement interactions; either due to high combative ability as associated with species from the secondary succession (Boddy 2000) or to better adaptation to the stressors present in these branches. Both branches were completely devoid of bark and there was evidence of extensive activity by wood-boring invertebrates which may compete with ligninolytic fungi for woody biomass (Harold et al. 2005; Tordoff et al. 2006; Crowther et al. 2012). C. gilvescens has previously been shown to be associated with ambrosia beetles (Ambrosiodmus / Ambrosiophilus spp.), a known vector of ligninolytic fungi into partially decayed substrata and with which C. gilvescens may be mutualistic (Kasson et al. 2016). Thus the likely scenario is that C. gilvescens dominated these branches not because of combative ability but because of its greater tolerance of the specific stressors acting on the community. Similar conclusions have previously been drawn on the presence of Schizopora paradoxa and Hyphoderma setigerum in attached branches of Q. robur (Boddy \& Rayner 1983a).

In summary this work has provided, for the first time, a quantification of the relative size of decay columns formed within woody substrata by mycelial colonies of ligninolytic fungi. This is an important metric of ligninolytic community composition and structure which influences outcomes of interactions between fungi and their environment and, at the pioneer stage, alters the trajectory of community assembly during later successional stages (Toljander et al. 2006; Falconer et al. 2011; Hiscox et al. 2017; O'Leary et al. 2018). Community structure was influenced to a greater extent by branch physical characteristics than by purely spatial variables at all taxonomic and functional levels. Wood mass loss, which is a fungal-driven process but also linked to nutrient profile and drying regime of the remaining lignocellulose resource, influenced observed variation in community structure to the greatest degree at lower taxonomic levels. At taxonomic levels higher than order level and at all functional levels, wood diameter, which is related to the gradient of temperature and moisture profiles, was the most important explanatory variable. No evidence of a SAR was found in mycelia associated with attached dead branches of F. sylvatica. As such, species richness and diversity in attached branches is likely driven by the stochastic processes which determine propagule distribution and abundance within healthy sapwood vessels.

In summary the structure and spatial configuration of pioneer ligninolytic fungi in surveyed beech branches was found to be influenced predominantly by environmental factors. In particular mass loss of the wood exerted a strong effect on the distribution and spatial arrangement of fungal decay regions 
within branches at species and order levels. At phylum level branch diameter become more important. Spatial auto-correlation was not determined to be the most important driver of community structure and spatial configuration of colonies within branches, although site-level spatial variables (longitude or latitude) tended to be fitted relatively early on in step-wise model building. This could relate to spatial and temporal auto-correlation of ambient climatic conditions, e.g. temperature, or could indicate a lasting effect of the spatial auto-correlation of the species pool exerted on the mycelial community through the distribution of propagules prior to community assembly. Interestingly, no SAR either positive or negative was detected as a function of either wood volume or lateral area. This suggests that larger branch size may not necessarily correlate with a propagule community richer in pioneer wooddecayer species; or at least that environmental filtering during assembly of the mycelial pioneer community prevents the establishment of greater numbers of pioneer species as mycelial colonies. Given that branch physical parameters were seen to exert stronger effects than spatial variables at all levels of taxonomy and functioning, environmental gradients are implicated in selecting for particular fungal traits as the wood varies in its ability to buffer against excessive drying or fluctuations in temperature and as the wood becomes more severely degraded. Thus, while combative competition for space may not result in changes to the spatial configuration of fungal colonies in attached branches over time, changing environmental conditions could result in a greater degree of partial-replacement interactions than has previously been acknowledged. While as much care as possible was taken to reduce the impact of pseudo-replication on the results of the study, they should be interpreted with caution and further replication introduced by repeating the surveying design at different sites.

The pioneer ligninolytic communities associated with dead attached beech branches are dominated by members of the orders Agaricales at all stages of decomposition, with the earliest stages of decomposition also being dominated by Xylariales and the most decayed branches also being dominated by Polyporales. The pioneer community in the branches surveyed degraded up to $75 \%$ of the original dry mass of wood. Once attached wood becomes very decayed, territory may be captured from original colonists by species that are given a competitive advantage by a biotic or abiotic stressor. Here, the most decayed branches were dominated by $C$. gilvescens which may have been better selected to withstand excessive invertebrate activity. 


\section{Chapter 4 - Differential effects of physical branch characteristics and community structure on enzyme activities and the metabolome of ligninolytic fungi in tree branches}

\subsection{Introduction}

Dead wood habitat plays a vital role in forest ecosystems as a major store of organic carbon owing to its resistance to, and therefore slow rate of, decomposition (Floudas et al. 2012). Dead attached branches, colonised by pioneer fungi, represent the beginning stages of most deadwood habitat creation (Boddy 2001; Boddy et al. 2017). The activities of these pioneer communities contribute significantly to overall wood degradation before well-decayed wood falls to the woodland floor (Chapter 3; Boddy \& Swift 1983; Harmon et al. 1986; Boddy 2001). Decomposition processes in angiosperm wood are driven almost exclusively by white-rot fungi through the secretion of powerful oxidative ligninolytic enzymes, such as laccase (phenyloxidase), lignin peroxidase, manganese peroxidase and manganese-independent peroxidase (Hofrichter et al. 2010), which break lignin polymers down into their monomeric compounds. Different white-rot species are known to differ in the range of ligninolytic enzymes they are capable of producing (Valmaseda et al. 1990; Eastwood et al. 2011) and in the identity and activity levels of enzymes expressed over time in relation to the initial capture of wood resource (Presley et al. 2018). In addition, white-rot fungi like all saprotrophic microbes secrete a range of hydrolase enzymes as they degrade various carbohydrates in their surroundings and in the search for limiting nutrients (Baldrian 2009).

Fungal-driven decomposition processes are complex and influenced by a wide range of environmental factors that fluctuate within wood including water content (Magan 2008; Hoppe et al. 2016) as well as the structural and chemical properties of wood and its cell walls (Schwarze et al. 2000; Schwarze 2007). In addition to mediating interactions between ligninolytic fungi and their abiotic environment, secreted enzymes are employed by fungi to mediate biotic interactions. The pioneer community initially colonises recently-dysfunctional wood through primary resource capture in an effective race for space (Boddy 2001; Boddy et al. 2017). Once the branch is fully colonised however, further resource can only be acquired through replacement interactions, wherein a part or all of a neighbour's territory is gained and their vegetative mycelia recycled by the captor (Boddy 2000; Hiscox \& Boddy 2017).

In dead attached branch communities, replacement interactions are not considered to be common. Instead, once primary resource capture is complete, neighbours within the community are hypothesised to be in relative deadlock with each other wherein both neighbours actively defend their territory either side of interaction zone lines but are not engaged in combative antagonism (Boddy \& Rayner 1983a; 
Boddy 2000, 2001; Boddy et al. 2017). The pioneer fungus Oudemansiella mucida is associated with a wider range of decomposition than any other pioneer species in attached beech branches (Chapter 3) and is known to be a particularly defensive species due to the production of a powerful antifungal strobilurin (oudemansin A; Anke et al. 1979). While pioneer fungi are known to be weak combatants against secondary successional species (Hiscox et al. 2016b; Toledo et al. 2016), they have generally been utilised experimentally as bait for more antagonistic fungi rather than being of interest themselves (Hiscox et al. 2010a). The pioneer species Vuilleminia comedens has been shown to be capable of replacing other pioneer species in wood (Hiscox et al. 2015b) and has even demonstrated considerable defensive capability against an aggressive combatant when given sufficient home advantage ( $\mathrm{O}^{\prime}$ Leary et al. 2018) indicating that the true nature of fungal-fungal interactions in the pioneer community are context dependent.

While there is extensive evidence for the up-regulation of oxidative enzymes during antagonistic interactions involving secondary and tertiary successional wood decay species (Freitag \& JJ 1992; White \& Boddy 1992; Iakovlev \& Stenlid 2000; Baldrian 2004; Hiscox et al. 2010a; Hiscox \& Boddy 2017), the role played by enzymes during mutually defensive interactions between pioneer fungi have never been studied. Activity levels of manganese peroxidase, manganese-independent peroxidase and particularly laccase increase within the deadlocking zone lines that form on artificial media between the earlysecondary decay species Trametes versicolor and other combative species compared with other parts of the colony of either species and with single species controls (Hiscox et al. 2010a). No other studies examining enzyme activity levels during interactions resulting in deadlock have been undertaken.

Differences in the activity levels of secreted enzymes will naturally impact upon the identity and concentration of the end products of fungal metabolism present as low molecular weight metabolites (small metabolites) in the surrounding substrate. To date, the effect of fungal-fungal interactions on the metabolome have only been investigated from the perspective of antagonistic combative interactions. Increased concentration levels of various metabolites involved in purine, pyrimidine, amino acid, central carbon, TCA, glucose, glyoxylate, and secondary metabolite metabolism have been reported from within interaction zone lines between T. versicolor and competitors compared with pure culture controls, although no comment was provided on whether interactions resulted in replacement or deadlock (Luo et al. 2017). Concentration levels of various unidentified metabolites along with erythritol, myo-insoitol and malic acid have been also been reported from interaction zones formed during replacement interactions involving S. hirusutm as the dominant competitor (Peiris et al. 2008). However there have been no studies investigating low molecular weight metabolite concentrations resulting from deadlock between any ligninolytic fungi. Following combative interactions with the late-secondary species Hypholoma fasiculare, the metabolome profile of T. versicolor in wood differs, particularly in the concentration levels of metabolites associated with alkaloid and coenzyme-A biosynthesis and aromatic hydrocarbon degradation, but not following replacement of the pioneer $V$. comedens indicating that at 
the metabolite level in wood, the relative combative strength of neighbouring fungi may be an important factor (O'Leary 2018). Concentration levels of sugar alcohols and trehalose, both of which have been linked with stress response, are increased during antagonistic interactions between wood-inhabiting fungi (Ocón et al. 2007; Peiris et al. 2008; Ujor et al. 2012).

Over time, as decay advances, the strategies employed by fungi to decompose lignocellulose, acquire nutrients and mediate their interactions are likely to change. These dynamics are not well understood in any dead wood system and have never been examined in pioneer communities. During primary capture of wood resource by the early-secondary decay species T. versicolor and S. hirsutum, activities of laccase and the hydrolases $\beta$-glucosidase and $\beta$-galactosidase are higher in younger parts of the colony, with hydrolase levels dropping steadily with increasing decomposition (Presley et al. 2018). Endochitinase, chitobiosidase and NAG activity levels also increase in older parts of the colony of many species of ligninolytic fungi following primary resource capture of woody resources (Lindahl \& Finlay 2006) indicating that hydrolase enzymes associated with $\mathrm{N}$ acquisition may increase over decomposition gradients as colonisation times increase. Conversely the activity levels of laccase and various hydrolytic CAZymes may fall over time as the resource becomes increasingly decayed.

In the present study, activity levels of secreted enzymes and metabolome profiles were examined in wood samples taken from decayed attached Fagus sylvatica branches. This is the first time that metabolomics has been applied to any wood decay system developing under natural woodland conditions. Untargeted metabolomics approaches were applied to facilitate future hypothesis generation. The enzymes assayed included a wide range of oxidoreductases utilised in lignin modification and hydrolases known to be utilised in the acquisition of nutrients by ligninolytic fungi (Baldrian 2009). There is, as yet, minimal understanding of the manner in which the suite of lignindegrading enzymes are employed to effect overall wood decomposition outside of biorefinery models (Zhang \& Schilling 2017; Presley et al. 2018). Attached dead branches, which represent the earliest stages of wood decay in natural woodland systems, are an ideal subject to explore this aspect of the functional ecology of wood decay. This work aimed to explore these dynamics in this system by asking two principal questions: (1) how do enzyme activities and metabolite identity and concentrations differ between colony regions nearer to and farther away from interaction zone lines? (2) how do enzyme activities and metabolite identity and concentrations change over the course of advancing decomposition of the wood substrate? 


\subsection{Outline of methods}

\subsubsection{General sampling methods}

Wood cores were extracted from whole branches colonies by pioneer communities from random locations adjacent to sampling locations used for community mapping (Fig. 2.2; Section 2.1.4; Chapter 3). Cores were split in half along their length, flash frozen and freeze dried prior to storage at $-80^{\circ} \mathrm{C}$. Total protein was extracted from one half of each core for enzyme assays (Section 2.3.1) and ACN : methanol : $\mathrm{H}_{2} \mathrm{O}$ metabolite extractions performed on the other half for untargeted GC-MS metabolomics (Section 2.4). Extractions were undertaken by agitating the wood in the appropriate extraction buffer to target the extracellular portions of the proteome and metabolome (Moody et al. 2018a). Samples were grouped according to whether they intercepted an interaction zone line between neighbouring fungi or not by comparison with photographs and community maps (Appendix I) and related back to the mass loss, water content and volume of the adjacent branch slice (Section 2.1.7).

\subsubsection{Quantitative enzyme assays}

Total protein was standardised to that of the least concentrated sample (Table 2.3). Laccase activity was determined by monitoring the activity of ABTS spectrophotometrically at $420 \mathrm{~nm}$, lignin peroxidase by monitoring the activity of veratryl alcohol following the addition of $\mathrm{H}_{2} \mathrm{O}_{2}$ at $310 \mathrm{~nm}$ and $\mathrm{MnP}$ by monitoring the colouration of oxidative-coupling of DMAB at $595 \mathrm{~nm}$. Results were corrected by activities of samples without manganese and with EDTA to chelate any $\mathrm{Mn}^{+2}$ present allowing detection of manganese-independent peroxidase. The oxidation of veratryl alcohol was followed to assay the activity of LiP (Section 2.3.4). Three technical replicates of each assay were performed for each sample.

Activity levels of hydrolytic enzymes were assayed by the addition of MUF and AMC linked-substrates and quantified by following fluorescence of samples over 120 min with an excitation wavelength of 355 $\mathrm{nm}$ and an emission wavelength of $460 \mathrm{~nm}$. Quantititive enzyme activity was defined as the amount of enzyme releasing $1 \mathrm{mM}$ of MUF or AMC $\mathrm{min}^{-1}$ (Section 2.3.3). Three technical replicates were performed for each sample.

\subsubsection{Untargeted GC-MS metabolomics}

Filtered extractives were dried in vacuo and derivatized using MSTFA+ 1\% TMCS with $10 \mu$ letracosane added as an internal standard (Section 2.4.1). Samples were injected in spitless mode at a temperature of $250^{\circ} \mathrm{C}$ onto a 5975 GC-MS (Agilent) with inert mass spectrometer detector and 5\% Phenyl Methyl Siloxane column (Section 2.4.2).

GC-MS data were pre-processed, deconvoluted and spectra compared with the NIST 11 (Wiley) and GMD (Kopka et al. 2005) spectral libraries. Putative identifications were based on matches of spectra 
with $\geq 80 \%$ similarity combined with visual inspection of automatically-matched spectra.

Chromatogram alignment, removal of internally-matching peaks and identification of both conserved and unique chromatogram features were undertaken in SpectConnect (Flory et al. 2007). Compound concentrations were normalised to the tetracosane internal standard (Section 2.4.3).

\subsubsection{Data analyses}

Analyses of enzyme activity levels were undertaken in R statistical software (R Studio Team 2016; 2018 R Core Team). Tests for differences in activity levels between samples intercepting a zone line (competing) and those further away from interaction zone lines (non-competing) were undertaken using Wilcoxon signed rank test. Spearman-rank correlations were performed to test for changes in activity levels of any of the enzymes assayed over the gradients of wood mass loss of the adjacent branch slice, water content of the adjacent branch slice, diameter of the adjacent branch slice branch volume, the size of the decay regions produced by the fungi within each sample determined by cross reference with community maps (Appendix I) and number of neighbours held by fungi within each sample determined by crossreference with community maps (Section 2.2.8).

Analysis of the metabolome profiles from wood cores were undertaken in MetaboAnalyst 4.0. (Chong et al. 2018). Separation of samples into competing and non-competing groups based on observed variation in compound identities and concentrations was visualised by PLS-DA. Q2 values were used to determine the predictive accuracy of models and important compound contribution to the separation of samples assessed by VIP analysis (Section 2.4.4.2). T-tests with a 5\% Benjamini-Hochberg FDR correction for multiple comparisons (Benjamini \& Hochberg 1995) were applied to test the null hypothesis of no significant differences in compound concentration between experimental conditions (Section 2.4.4.2).

Lipids are important metabolites for the storage of carbon and energy in all biological systems. In addition sugar alcohols have been found to be important during interspecific combative interactions between ligninolytic and other saprotrophic fungi (Hiscox \& Boddy 2017) and the availability of the major sources of carbon in decaying wood, such as cellulose, may change predictably as decomposition advances. The concentrations of metabolites identified from these chemical classes were examined over the decomposition gradient (average mass loss of wood within the branch). Statistical analyses were performed in R Studio. Spearman-rank correlations were computed to test the null hypothesis that no correlation was present between wood mass loss and the metabolite of interest.

To examine whether the observed variation in the metabolome of pioneer fungi colonising wood cores was related to the observed variation in enzyme activity levels, to community dynamics of wood physical properties data were imported into PRIMER 7 and distLM models built using (1) wood and fungal community parameters, and (2) enzyme activity levels as predictor variables. Models were built on resemblance matrices of the metabolome with either the branch environment or enzyme activity 
levels variables as predictor variables and the following parameters: no grouping of predictor variables by indicators; a step-wise selection procedure; adjusted R² selection criterion; obtain P-values by 999 permutations; run marginal tests; and plot the model as a dbRDA plot. Significance level was deemed to be $\mathrm{P} \leq$ 0.05. (Section 2.4.4.4).

\subsection{Results}

\subsubsection{Sampling attached branch communities for enzyme activity and metabolomic profiling}

The enzyme study comprised 19 samples (Table 4.1): nine were found to have intersected the zone lines between two neighbouring fungi (competing); a further 10 were found to have intersected an area of a colony formed by a single species not immediately adjacent to a neighbouring competitor (noncompeting). Unfortunately, seven samples prepared for metabolomics were not injected correctly by the GC-MS autosampler and were lost. As such the metabolomics study comprised 12 samples, 5 colonised by competing fungi and 7 colonised by non-competing fungi (Table 4.1). The decomposition gradient ranged between $4.17-76.39 \%$ mass loss and the moisture gradient between $21.3-83.3 \mathrm{~g} \mathrm{~cm}^{-3}$. When grouped into non-competing and competing samples, mass loss had means of $41.8 \%$ and $39.82 \%$ while water content had means of $37.93 \mathrm{~g} \mathrm{~cm}^{-3}$ and $41.62 \mathrm{~g} \mathrm{~cm}^{-3}$ in the non-competing and competing groups respectively. O. mucida, $H$. cohaerens and C. gilvescens were present in both non-competing and competing groups but other species were only represented in one condition or the other. All species colonising branch slices adjacent to core sampling locations were white rot species. 
Table 4.1 Properties of wood core samples and, their adjacent branch slices

Slice, labels of branch slices adjacent to enzyme/metabolite sampling locations (Chapter 3 and Appendix I); Mass Loss, mass loss calculated for the adjacent wood slice; $\mathrm{H}_{2} \mathrm{O}$, water content calculated for adjacent wood slice; $\varnothing$, diameter of the adjacent wood slice; colony size calculated by adding the total number of cells occupied by the colony or colonies present in the sample across all slices in which they were identified (Chapter 3 and Appendix I); neighbours calculated by counting the number of other colonies in the branch neighbouring the colony/colonies of interest.

\begin{tabular}{|c|c|c|c|c|c|c|}
\hline Slice & $\begin{array}{c}\text { Mass } \\
\text { Loss, \% }\end{array}$ & $\begin{array}{c}\mathrm{H}_{2} \mathrm{O}, \\
\mathrm{g} \mathrm{cm}^{-3}\end{array}$ & $\varnothing, \mathrm{mm}$ & $\begin{array}{c}\text { Colony } \\
\text { Size }\end{array}$ & Neigh. & Study \\
\hline CLY03C & 4.17 & 25.8 & 10.5 & 108 & 11 & Enz; Metab \\
\hline CLY03D & 20.84 & 23.6 & 7.5 & 21 & 6 & Enz; Metab \\
\hline CLY19C & 36.12 & 22.6 & 6.45 & 15 & 7 & Enz; Metab \\
\hline CLY03B & 40.28 & 25.1 & 13.35 & 51 & 7 & Enz \\
\hline CLY19E & 44.45 & 24.4 & 6.45 & 15 & 7 & Enz; Metab \\
\hline CLY04C & 45.84 & 50.5 & 9.5 & 35 & 6 & Enz; Metab \\
\hline CLY04A & 47.23 & 38 & 11.75 & 110 & 5 & Enz; Metab \\
\hline CLY04B & 48.62 & 43.6 & 9.5 & 110 & 5 & Enz; Metab \\
\hline CLY07C & 54.17 & 46.8 & 10.5 & 385 & 17 & Enz \\
\hline CLY07G & 76.39 & 78.9 & 6.95 & 78 & 7 & Enz \\
\hline CLY13C & 9.73 & 27.1 & 17.65 & 418 & 1 & Enz; Metab \\
\hline CLY03A & 20.84 & 30.2 & 16.85 & 35.5 & 6 & Enz \\
\hline CLY13E & 29.17 & 27.1 & 15 & 418 & 1 & Enz; Metab \\
\hline CLY19A & 38.89 & 35.7 & 7.5 & 42.5 & 7 & Enz; Metab \\
\hline CLY13G & 40.28 & 21.3 & 11.6 & 418 & 1 & Enz; Metab \\
\hline CLY12B & 43.06 & 56.7 & 6.45 & 30 & 14 & Enz \\
\hline CLY03E & 55.56 & 40.2 & 3.8 & 1.5 & 3 & Enz \\
\hline CLY04D & 56.95 & 53 & 13 & 38.3 & 8 & Enz; Metab \\
\hline CLY07E & 63.89 & 83.3 & 14.5 & 203 & 17 & Enz \\
\hline
\end{tabular}

\subsubsection{Oxidative and hydrolytic enzyme activity levels of competing and non- competing fungi}

With the exception of MiP, all enzymes assayed were detected in the study. LiP was detected in nine samples. There were no significant differences in activity levels of any of the enzymes assayed between competing and non-competing fungi (Wilcoxon-signed rank test; $\mathrm{P}>0.05$ ). LiP was detected in $30 \%$ of samples in the non-competing group and $86 \%$ of samples in the competing group (two samples in the competing group, CLY03E and CLY13C could not be assayed due to insufficient sample volume (Appendix II). Mean activity levels of lignin peroxidase $(\mathrm{P}=0.093)$, manganese peroxidase $(\mathrm{P}=0.066)$ and leucine aminopeptidase $(\mathrm{P}=0.079)$ were higher in the competing group but the correlations were non-significant. 


\subsubsection{Correlations of oxidative and hydrolytic enzyme activity levels with branch quality factors}

The activity levels of eight enzymes were found to be significantly correlated with mass loss $(\mathrm{P} \leq 0.05$;

Table 4.2). Laccase correlated positively with mass loss while manganese peroxidase, $\beta$-glycosidase, cellobiohydrolase, endoxylanase, $\mathrm{N}$-acetylglucosaminidase, arylsulfatase and phosphodiesterase activity levels fell significantly as mass loss increased $(\mathrm{P} \leq 0.05)$. Laccase and $\mathrm{N}$-acetylglucosaminidase were found to only correlate significantly with mass loss among the independent variables. The activity levels of four enzymes ( $\beta$-glycosidase, endoxylanase, arylsulfatase and phosphodiesterase) were found to have significant negative correlations with moisture content of the surrounding wood $(\mathrm{P}<0.05)$. A single enzyme, $\beta$-glycosidase, correlated negatively and significantly with the number of neighbours connecting with the sampled colony $(\mathrm{P}<0.05)$. There were no significant correlations between any of the enzymes assayed and either colony size or the diameter of the branch at sampling location.

\subsubsection{Untargeted GC-MS metabolomics of attached branch communities}

SpectConnect identified a total of 416 unique compounds present in 12 samples. Of these, 37 were putatively identified and matched to metabolites including 7 organic acids, 6 sugar alcohols, 6 fatty acids, 5 amino acids, 2 sugar acids, 2 saccharides, 3 phenols, and one each of amides, aromatic alcohols, mineral acids, nucleic acids, steroids and vitamins. Comparison with GMD and KEGG databases identified 15 metabolites involved with carbohydrate metabolism, 7 with amino acid metabolism, 4 with lipid metabolism, 4 with energy metabolism, 3 with polyphenol degradation, 2 with nucleotide metabolism and 2 with biosynthesis of secondary metabolites (Appendix VI).

\subsubsection{Differential metabolomics of attached branch communities}

Profiles were separated by PLS-DA into competing and non-competing groups with $35.1 \%$ and $17.6 \%$ of variation in the metabolome being explained by the first two components (Fig. 4.1). Four components were needed to build the optimum model $(\mathrm{R} 2=1.00 ; \mathrm{Q} 2=0.35 ;$ Accuracy $=0.75)$. The identity and concentration of 85 compounds were important in separating samples according to the presence or not of competing interactions (VIP Analysis; Fig 4.2). The top 12 most important compounds were unidentified but important compounds also included the putatively identified metabolites itaconic acid, cellobiose, phenylpyruvic acid, succinic acid, D-citramelic acid, pantothenic acid, arabitol, stigmastanol, xylobiose and a compound that matched an unknown analyte in GMD (Appendix II). 


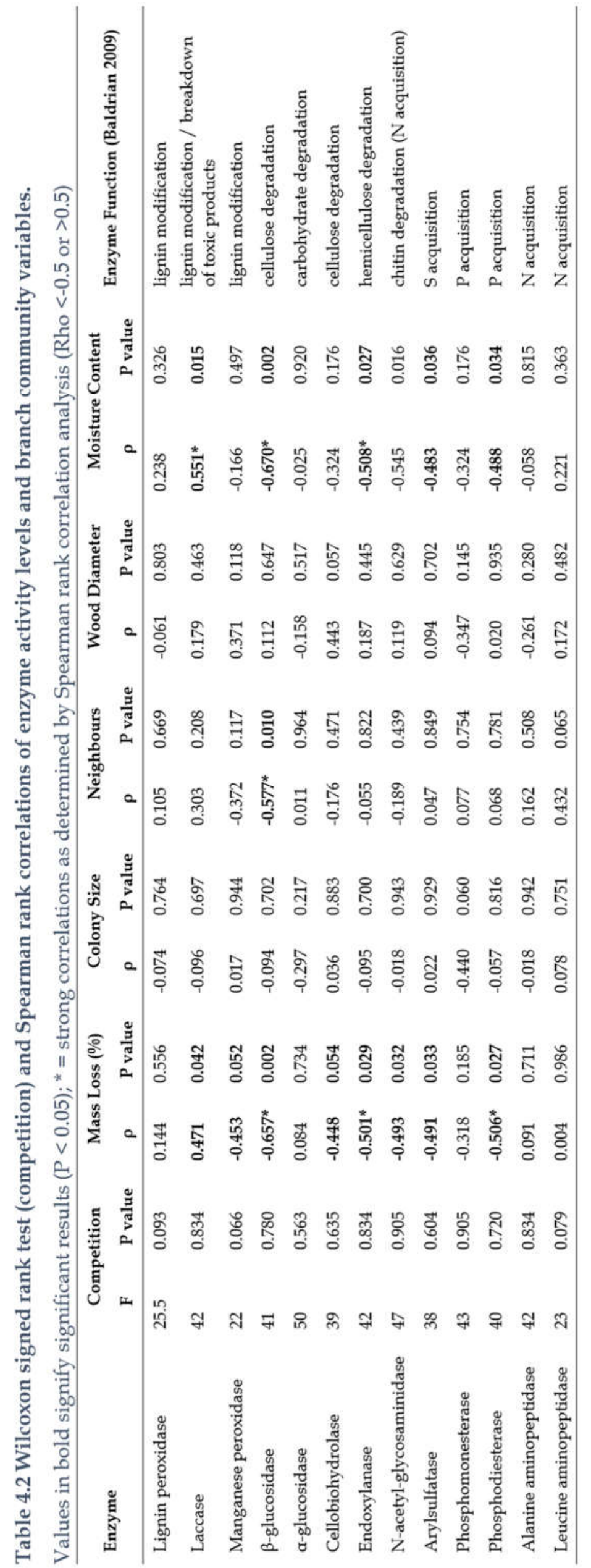

Page | 64 


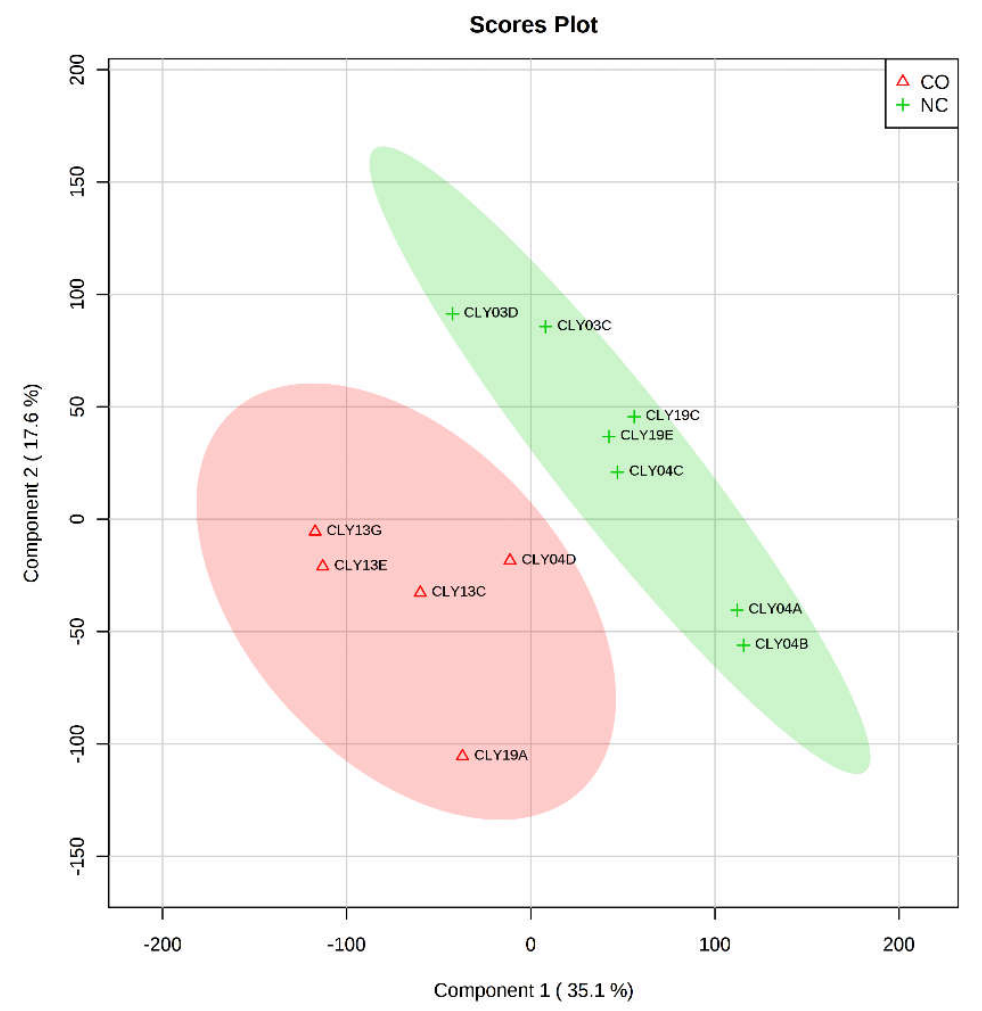

Figure 4.1 PLS-DA of the metabolome of fungal residents in branch wood cores.

Red, samples that intersected an interaction zone line between neighbouring decay regions produced by different fungal species ('competing'); green, samples that intersected a single decay region away from an interaction zone line ('not competing'); shaded areas, 95\% confidence limits.

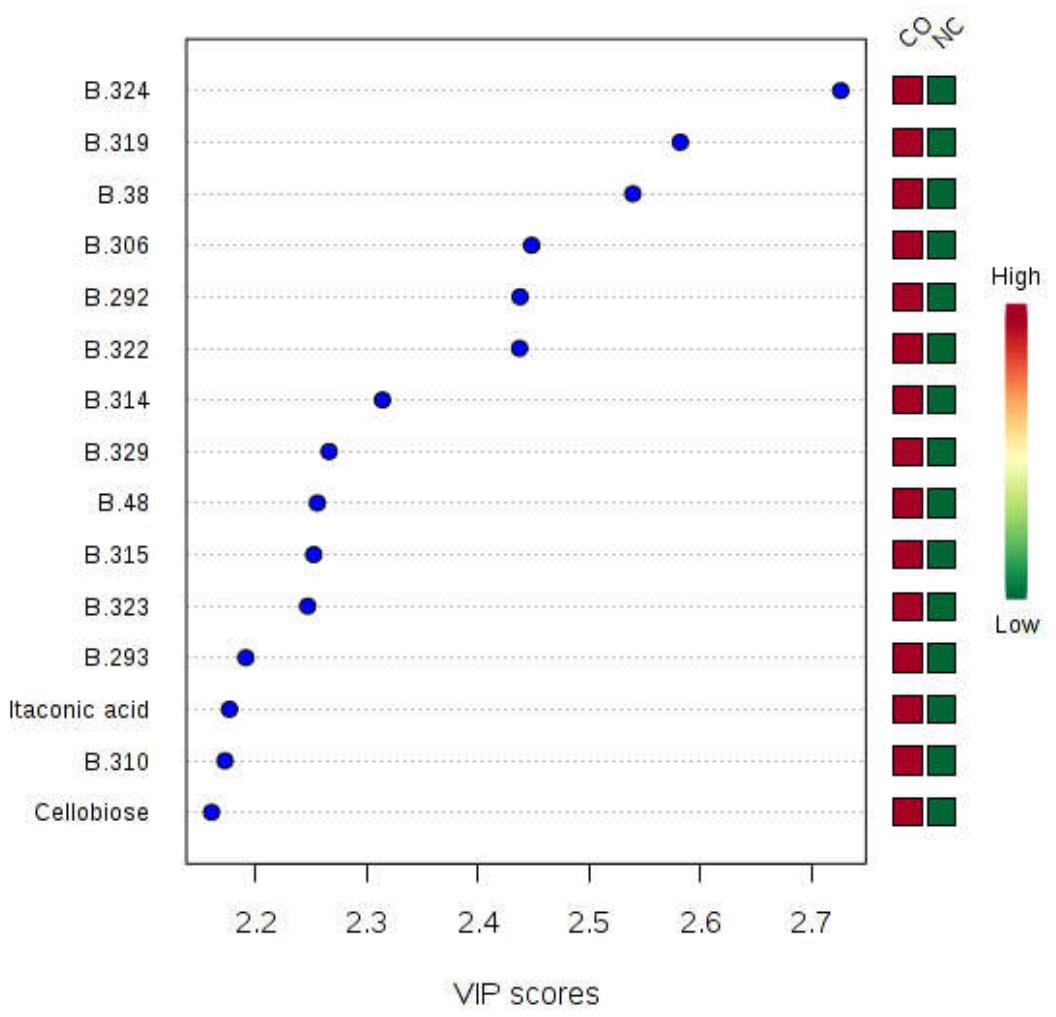

Figure 4.2 Top 15 compounds contributing to the separation of samples into treatment groups in PLS-DA

Red, compound present in greater concentration in treatment; green, compound present in lower concentration in treatment; $\mathrm{CO}$, samples that intersected an interaction zone line between neighbouring decay regions produced by different fungal species ('competing'); NC, samples that intersected a single decay region away from an interaction zone line ('not competing') 
No compounds were found to differ in concentration between competing and non-competing fungi ( $\mathrm{T}$ tests; P $\geq 0.05$; FDR 0.05; Appendix VII). Among those compounds exhibiting at least \pm 2.0 -fold change (FC) in concentration in competing compared with non-competing fungi, the majority increased in concentration (Fig. 4.3) in mean concentration between competing and non-competing fungi. Putatively identified metabolites with FC $>2.0$ under the competing condition included cellobiose $(\mathrm{FC}=53,022)$, pantothenic acid $(\mathrm{FC}=38,645)$, phenylpyruvic acid $(\mathrm{FC}=8.76)$ and succinic acid $(\mathrm{FC}=7.56)$. Those with FC $<-2.0$ included the putatively identified metabolite stigmastanol ( $\mathrm{FC}=5.48^{-5}$; Appendix II). There were no correlations between the concentration levels of commonly detected lipids (fumaric acid, palmitic acid and stearic acid), sugar alcohols (glycerol, sorbitol, erythritol and myo-inositol) or saccharides (cellobiose and xylobiose) and mass loss of the surrounding wood (Spearman-rank correlations; P > 0.05; Appendix II).

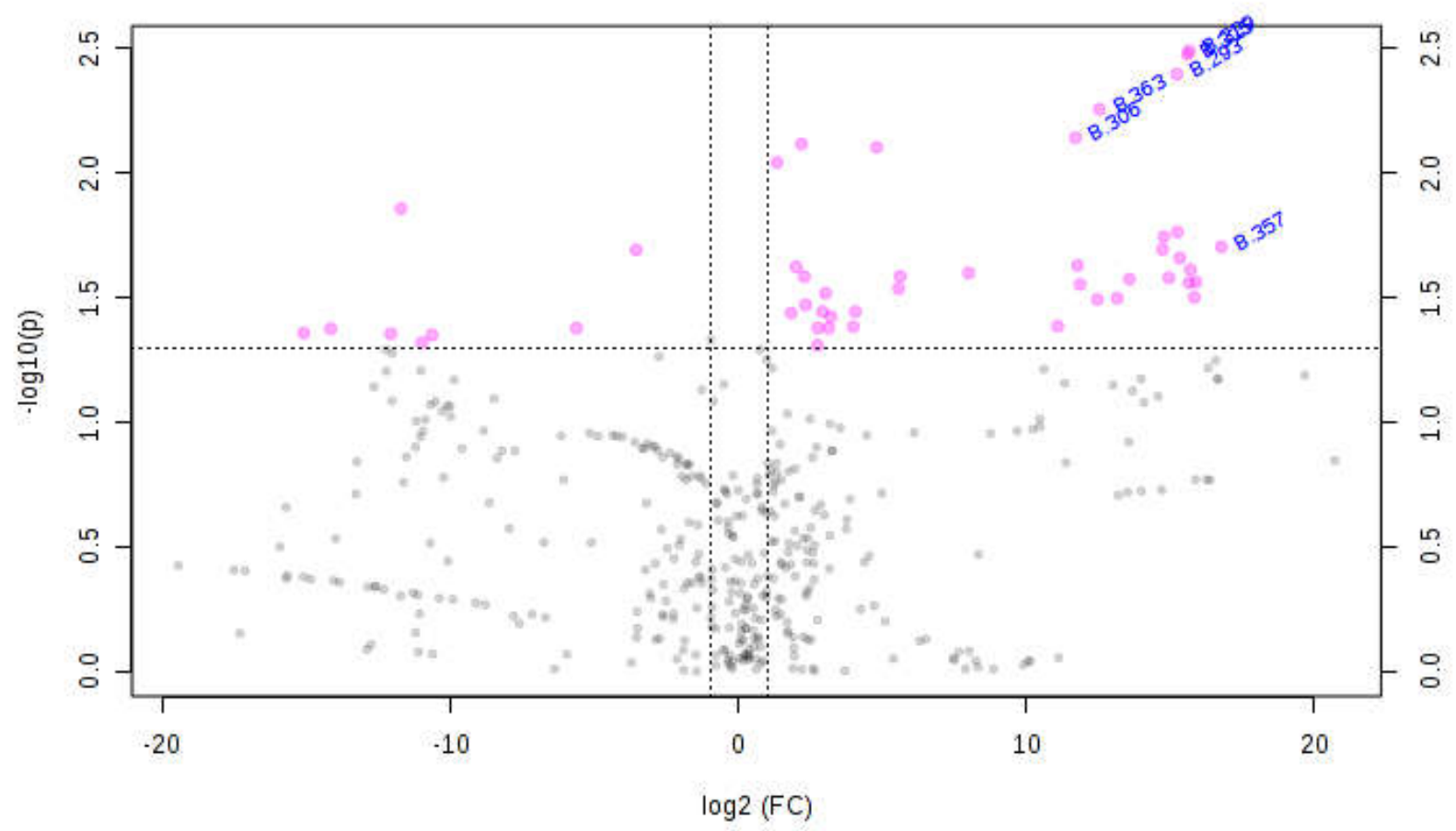

Figure 4.3 Volcano plot of compounds present in samples intersecting interaction zone lines compared with samples intersecting single colonies in attached branches

Pink, compounds with concentration exhibiting FC < -2 (left) or > 2 (right); blue labels, compounds exhibiting significantly increased concentration in samples intersecting an interaction zone line between decay regions produced by different fungi ( $\mathrm{T}$ test; raw P-value; FDR: 0\%)

\subsubsection{Distance based linear modelling of attached branch metabolomes, microhabitat factors and enzyme activity levels}

A distLM model comprising wood water content AND 'competition' covaried with metabolome structure, explaining $16.2 \%$ of the observed variation (distLM; P - 0.04; Appendix VIII). Laccase activity 
level was determined to be the only predictor variable to covary significantly with metabolome profile and explained $20.8 \%$ of the observed variation in the metabolome (distLM; P = 0.022; Appendix VIII).

\subsection{Discussion}

The activities of several enzymes correlated with mass loss and water content of the wood. Most were strongly negative $(\rho<-0.5)$. This is the first time that natural decomposition processes of the resourcerestricted pioneer wood degrading community in attached dead branches have been reported. The mass loss gradient studied here, which ranged from $4.17 \%$ to $76.39 \%$ of dry mass loss compared with healthy sapwood ( $0 \%$ mass loss), likely represents a period of several years' decomposition (Chapter 3). Enzyme activities observed were highly variable which is not an uncommon feature of environmental wood samples (Valášková et al. 2009; Arnstadt et al. 2016; Noll et al. 2016). Nevertheless, the results presented here provide a view of some of the natural long-term changes in investment by the fungi involved.

Of the lignin transforming oxidoreducatses, manganese peroxidase activity was observed to decrease with increasing mass loss. This is consistent with previous work which has shown that, over 42 weeks following supply of fresh uncolonized wood, white-rot fungi invest early on in the secretion of peroxidases (Kuuskeri et al. 2016) and then later in increased activity and accumulation of carbohydrate active hydrolases (Hori et al. 2014; Presley et al. 2018). Here however, laccase increased with increasing mass loss which contrasts with the findings of laboratory scale studies (Kuuskeri et al. 2016; Presley et al. 2018) and field studies following enzyme activities in CWD over a 6.5 year period (Kahl et al. 2017). Mass loss of the surrounding wood in attached branch communities is related to advancing depolymerisation of the lignin matrix, with cellulose becoming increasingly accessible as decay advances, and therefore to a change in the nutrient profile. As such, this observed increase in laccase may be linked with an increasing 'opening up' of the lignocellulose matrix and increased access to cellulose. Laccases are known to have multiple functions in fungi including stress defence, detoxification activities and morphogenesis as well as lignin depolymerisation however (Thurston 1994; Baldrian 2006). Thus the increasing investment in laccase observed here over time (increased mass loss) may also be a consequence of an increasingly toxic environment brought about by the production of reactive oxygen species and phenolics during wood decomposition (Moody et al. 2018a). Within heavily decomposed wood, the activity of bacteria and invertebrate species increases both of which produce laccases. However bacteria are thought to be relatively uncommon sources of environmental laccases and the same is likely to be true for invertebrates (Arakane et al. 2005; Baldrian 2006; Chauhan et al. 2017).

Strong negative correlations were observed between wood mass loss and cellulose degrading $\beta$ glucosidases, hemicellulose degrading endoxylanases and phosphodiesterases involved in P acquisition. The cellulose degrading cellobiohydrolases, chitin degrading $\mathrm{N}$-acetylglucosaminidases and arylsulfatases involved in $\mathrm{S}$ acquisition exhibited weak negative correlations with wood mass loss. These 
results contrast with laboratory scale studies which have found white rot fungi to invest highly in carbohydrate active hydrolases later in the decomposition process (Hori et al. 2014; Presley et al. 2018). However in Phlebia radiata, a white rot corticoid fungus associated with attached branch communities (Boddy 2000), the abundance of carbohydrate active enzymes (CAZymes) associated with cellulose degradation were reported to fall moderately over 42 days and while moderate increases in CAZymes associated with hemicellulose degradation and chitin degradation over the same period were reported, trajectories were not linear (Kuuskeri et al. 2016). It is worth noting however that higher protein abundance does not necessarily correlate with higher enzymatic activity (Zhou et al. 2018). In the field, strong positive correlations have been found between activity levels of lignocellulolytic enzymes and greater mass loss in logs left to degrade naturally over several years and these results seem to contrast sharply with the results presented here (Kahl et al. 2017). Larger scale wood sampling campaigns however have reported the high variability of secreted enzyme activities in wood and concluded that enzyme secretion patterns are dictated by a complex array of wood property and other environmental variables that are hard to encapsulate and measure at relevant scales (Arnstadt et al. 2016).

The activities of four hydrolytic enzymes involved in (hemi)cellulose degradation and acquisition of $S$ and $\mathrm{P}$ correlated negatively with moisture content which was again unexpected, although there was a strong positive correlation between laccase and water availability. Water content was the only significant driver in the observed variation in the metabolome of fungi in attached branch communities and so water content is clearly an important driver for some metabolic processes at protein and metabolite levels. In soil microcosms, both hydrolytic and oxidoreductive enzyme activities have been shown to correlate positively with moisture availability and that increased moisture availability buffered the negative effects of non-optimum temperature (A'Bear et al. 2014b). In attached branches water availability is considered to be at an extreme in the earliest stages with dysfunctional wood drying out naturally over time (Boddy et al. 2017). As decomposition advances the matrix of the wood opens up and so water availability will become subject to greater fluctuations. There is a need to examine these dynamics and their effect on fungal activity more closely in wood. Positive correlations have been recorded between water content and general peroxidases, but not laccase or manganese dependent peroxidase, in CWD of F. sylvatica, Pinus abies and Pinus sylvestris under field conditions (Arnstadt et al. 2016). Here, water content was calculated from the dried weight (dried at $65^{\circ} \mathrm{C}$ over 72 hours) of the adjacent branch slice following removal of its bark divided by its volume. Volume was calculated by measuring the outer dimensions of the wood slice. These methods may not have been precise enough to calculate moisture content at a scale relevant to the fungi. However Arndstadt et al. (Arnstadt et al. 2016) also reported unexpected correlations between enzyme activities and moisture content despite taking measurements from a standardised volume of wood. Enzyme secretion and activity levels are dictated by a complex array of variables but the need to better understand the dynamics between secreted 
oxidative and hydrolytic enzymes employed in wood degradation and the availability of moisture is clear.

The results presented here show that activities of hydrolase enzymes in attached dead branches may fall over the long term. While wood decaying species differ in their mechanistic abilities to degrade lignin (Floudas et al. 2012), hydrolase activity is necessary for the continuous acquisition of C, N and other limiting nutrients which are prerequisite for basic metabolic processes such as energy metabolism. As such these results are surprising. This was even true in the case of NAG (exochitinases) which was particularly unexpected. The activity of some chitinolytic enzymes, specifically endochitinases and chitobiosidases, have been reported to be higher under primary resource capture than under secondary resource capture in wood indicating that recycling of a colony's own cell walls is an important source of $\mathrm{N}$ in the pioneer community (Lindahl \& Finlay 2006) and as colonies age, these activities might be expected to increase. Furthermore, fungal biomass might be expected to accumulate over time as a result of wood becoming better colonised and hydrolase activities have been shown to correlate to some degree with fungal biomass (Noll et al. 2016). However C:N ratios have been reported to increase with increasing wood decomposition, driven by an accumulation of $\mathrm{N}$ and an increase in the proportion of lignin and moisture within wood (Arnstadt et al. 2016). These dynamics may partially explain the results reported here. Furthermore, this is the first time that enzyme activities in natural pioneer communities have been examined and the results presented here may not be directly comparable with those in woody debris where abiotic conditions are very different and where competition for space is known to be much more aggressive (Boddy et al. 2017). There is a clear need for future studies to provide much-needed replication and to build up a more complete picture of how these complex dynamics are influenced by biotic and abiotic conditions within natural wood decay communities at all successional levels. No correlations between enzyme activity levels and wood diameter were reported. Thinner sections of wood will be subjected to greater fluctuations in temperature and, particularly in more decayed wood, water content. In thicker sections of wood there will be a stronger gradient of both temperature and water content from the middle to the outer regions of the branch with inner regions maintaining more stable moisture levels and temperature. As such the lack of correlations with diameter and enzyme activity reported here suggest that enzyme activity levels suggest that activity levels may be driven by nutrient acquisition to a greater degree than by microclimate conditions.

Activity levels of oxidative and hydrolytic enzymes did not differ between regions of decay columns closer to interaction zone lines and regions farther away from interaction zone lines. These results may indicate that observed differences in enzyme activity levels in natural pioneer communities are a product of nutrient acquisition processes rather than a consequence of mutually defensive biotic interactions. It is interesting to note that, while no statistical difference in activity levels of lignin peroxidase was determined between competing and non-competing fungi, lignin peroxidase was 
detected in $86 \%$ of samples intersecting an interaction zone line but only $30 \%$ of samples taken from further away from zone lines. During interactions between combative antagonistic species, laccase activity increases in the interaction zone lines formed between them due to the accumulation of stable enzymes rather than an increase in expression of laccase producing genes (Hiscox et al. 2010a). As such, the presence of lignin peroxidase in a greater proportion of 'competing' samples compared with 'noncompeting' samples observed in the present study may be more informative of fungal strategy.

In the metabolomes of fungi degrading attached dead branches, no significant differences were found between competing and non-competing fungi and this could again have been a consequence of sampling technique. The abundances of five putatively identified metabolites did exhibit large $(>2.0)$ fold changes under the two conditions however. Cellobiose, pantothenic acid, phenylpyruvic acid and succinic acid were observed to increase in abundance in competing fungi while the plant sterol stigmastanol decreased in abundance. Pantothenic acid is an essential nutrient (Vitamin $B_{5}$ ) and a pre-cursor to coenzyme-A (CoA; White et al. 2001; Leonardi et al. 2005) and as such to metabolic pathways associated with energy metabolism. Succinic acid also plays a significant role in energy metabolism and in the response to stress (Tretter et al. 2016). Phenylpyruvic acid is involved in amino acid metabolism (phenylalanine metabolism) and the biosynthesis of secondary metabolites (Kanehisa \& Goto 2000) and has been long known as an intermediate product of lignin degradation (Fukuzumi 1960). Collectively these results suggest a number of putatively identified metabolites utilised by fungi in attached branch communities to increase investment in respiration and lignin degradation in response to competition from neighbouring colonies. These results may inform future, more targeted studies of the metabolic processes and pathways involved in competitive and non-competitive interactions of wood degrading fungi associated with the primary decay community and attached dead branches.

It is important to note that the activities of cutting an attached branch away from its host tree and dividing the resource into slices of wood (Chapter 3) will naturally affect fungal metabolic processes, including enzyme activities and expression of small metabolites. As such it was decided that wood sample extraction and preservation for the present study should be the first priority and an increment borer was utilised to extract wood samples from the branch while still whole to achieve this. Wood slices were later removed from regions of the branch adjacent to the core sampling locations to facilitate identification of the fungi within each core. As such the sampling regime could not consider the identity and configuration of decay regions and interaction zones a priori and may not have been sensitive enough to support the separation of samples into competing and non-competing regions of the decay columns. Even cores colonised by multiple species often necessarily comprised much more wood taken from deep within the colony than wood directly adjacent to the delimiting boundaries between neighbours. The activity of secreted enzymes employed by wood degrading fungi are highly localised within colonies (Lindahl \& Finlay 2006; Šnajdr et al. 2011) with observable differences between activity 
levels in colony regions just behind interaction zone lines and within the zone lines themselves (Hiscox et al. 2010a) which is also likely to drive similarly detectable differences in metabolome. However, the present study is the first to attempt to relate observed changes in fungal metabolism to aspects of the ecology of mycelial colonies within decaying wood and has proven that, while some refinements could be made, such studies can produce highly informative observations.

In summary there was evidence that fungal metabolism in pioneer ligninolytic communities may be driven to a greater extent by the search for nutrients than by either microclimate conditions or biotic interactions, supported by correlations between enzyme activity levels and mass loss of wood but not wood diameter and a lack of influence of interaction zone lines in samples. An exception to this was water content of the wood which correlated negatively with activity levels of several hydrolases and drove variation in the identity and concentration of the metabolome. The mainstay of experimental work investigating metabolic processes in wood decay systems manipulate temperature or biotic factors. While moisture levels are harder to control in wood, there is a clear need to investigate the role of water availability and the functional aspects of wood decomposition. 


\section{Chapter 5 - Untargeted metabolomics and proteomics reveal lasting effects of early-arriving species and wood anatomy on the metabolism of Exidia glandulosa}

\subsection{Introduction}

The establishment, growth or reproduction success of species associated with secondary and tertiary successions can be significantly affected by priority effects exerted by the first-arriving organisms. These effects have been found to be responsible for differences in both the structure and functioning of ecosystems (Dickie et al. 2011; Tan et al. 2012), sometimes exerting a greater effect than abiotic conditions (Fukami 2015). Understanding the mechanisms by which early-arriving species exert their influence poses a serious challenge to community ecology because in many systems, acquiring the relevant historical information is impossible (Fukami 2015). One of the central goals in the study of ecological communities however, is to understand the mechanisms underlying their assembly (Gleason 1927).

To date, there have been very few published attempts to examine the influence priority effects may have on organismal biochemistry even though an organism's establishment, growth and reproductive success is mediated through its biochemistry. Priority effects have been studied at the genome level in nectar yeast communities (Dhami et al. 2016) and at the transcript level in ectomycorrhizal fungi (Kennedy et al. 2009). A Scopus search with the parameters "priority effects" OR “assembly history" AND "metabolomics" OR "proteomics" OR “omics" returned no results (search conducted $13^{\text {th }}$ November 2018). In contrast, a Scopus search with the parameters "priority effects" OR "assembly history" limited to biological sciences subject areas returned 486 papers (search conducted $13^{\text {th }}$ November 2018). A search with the parameters "priority effects" OR "assembly history" AND “biochemistry" returned a single paper (search conducted 13th November 2018; Cline \& Zak 2015) in which substrate biochemistry and initial fungal colonist identity were manipulated in leaf litter decomposition systems. Therein it was found that physiological traits of the initial colonists, growth rate and ligninolytic potential, significantly altered community assembly trajectories.

Wood decay systems offer a unique opportunity to study the mechanisms of priority effects. Priority effects are often mediated by competition (Tan et al. 2012) and the wood matrix retains the profile of secreted proteins and metabolites utilised in fungal-fungal and fungal-wood interactions which can be profiled using approaches such as untargeted proteomics and metabolimics. Priority effects have previously been shown to influence community assembly in wood decay systems (Hiscox et al. 2015a; Van der Wal et al. 2016). Furthermore, the presence of endophytic species and a developed pioneer 
community has been demonstrated to significantly alter community assembly trajectories and to enhance decay rates in the secondary succession compared with situations where primary resource capture is undertaken by fungi associated with the secondary succession (Cline et al. 2018). These scenarios play out in attached dead branches and healthy wood downed by storm damage respectively and this work demonstrates the need to understand the molecular mechanisms by which early-arriving species exert their influence.

Partially-decomposed substrates will vary physically and chemically, in part due to the identity and phenotype of the initial microbial colonists but also because changes in biotic and abiotic pressures over time or between habitat patches will evoke changes in primary and secondary metabolism (Boddy \& Heilmann-Clausen 2008). To establish successfully, an invading colonist must be better able to cope with the biochemical landscape produced by its predecessor than its competitors (Heilmann-Clausen \& Boddy 2005). Whether replacement interactions between ligninolytic fungi take place along or across the grain of the wood (xylem vessels) significantly affects the proportion of resource successfully captured from the earlier colonist ( $\mathrm{O}^{\prime}$ Leary 2018). The three-dimensional nature of natural wood decay communities (Chapter 3) and the requirement to invade and defend territories across the wood vessels likely promotes coexistence and species richness in ligninolytic communities, particularly in the early stages when wood anatomy is still relatively intact, for similar reasons as increased viscosity of culture medium promotes coexistence in predator-prey ciliate communities (Luckinbill 1973). Thus, wood anatomy and the spatial complexity of the lignocellulose resource may affect fungal metabolism following replacement interactions and may therefore influence priority effects.

Increasing attention is being paid to the metabolic effects of competitive interactions largely from the perspective of targeted assays of enzymes (Eyre et al. 2010; Hiscox et al. 2010a, b) and known secondary metabolites (Peiris et al. 2008; Luo et al. 2017). Initial investigations of changes in expression of the total metabolome in artificial media (Peiris et al. 2008; Luo et al. 2017; Xu et al. 2018) and in wood (O'Leary 2018) as a result of ongoing interspecific interactions have been conducted. No attention has been paid however to any lasting effects that past competitive interactions might have on the metabolism of wood decaying fungi even though it is these effects that are likely to underpin on-going processes such as wood decomposition rate, community assembly trajectory and priority effects. Order of arrival of members of the pioneer community is known to be an important factor in the impact priority effects have on the community assembly trajectory of later successional stages (Frisch \& Green 2007; Louette \& De Meester 2007; Louette et al. 2008; Allen et al. 2011; Audet et al. 2013).

Untargeted metabolomics and proteomics approaches were employed to investigate the role of initial colonist identity and wood structural anatomy on the molecular response of an invading pioneer organism. Exidia glandulosa is an endophyte of wood decay systems and often a constituent of pioneer communities in beech wood (Heilmann-Clausen 2001; Heilmann-Clausen \& Christensen 2004). A 
member of the Auriculariales, E. glandulosa has a stress-selected life history strategy, is tolerant to desiccation and often found in sun-baked or other highly desiccated wood (Boddy \& Rayner 1983b; Boddy 2001; Heilmann-Clausen 2001). E. glandulosa will therefore likely out-compete other natural members of the primary decay community, such as Oudemansiella mucida and Stereum rugosum, under desiccated conditions. Metabolomics and proteomics analysis was undertaken on desiccated wood that had been completely recaptured by E. glandulosa from O. mucida and S. rugosum compared with selfpairing controls that had undergone primary resource capture only. The aim of the work was to: (1) determine whether the identity of initial colonists have a lasting effect on the metabolism of a laterarriving species; and (2) whether identity of the earlier-arriving species and wood structural complexity in relation to the orientation of replacement interactions have an additive effect on fungal metabolism.

\subsection{Outline of methods}

\subsubsection{Experimental design and microcosm conditions}

Three fungal pioneers isolated from attached branch communities (Chapter 3) were selected due to their ecological roles. The pioneer fungus E. glandulosa was chosen as the later-arriving species. E. glandulosa due to its association with desiccated conditions (Boddy \& Rayner 1983b; Boddy 2001; HeilmannClausen 2001). O. mucida was chosen as an earlier-arriving species due to its prevalence in the naturally formed communities previously surveyed (Chapter 3) and due to its highly defensive strategy (Anke 1995). S. rugosum was also selected as a later-arriving species due to its prevalence in attached branch communities but also because it is not particularly well known for being defensive. Thus E. glandulosa could be given a competitive advantage over the two early-arriving species in the experiment through the provision of desiccated conditions.

Sterile beech wood blocks measuring $2 \mathrm{~cm}^{3}$ were pre-colonised by placing on $0.5 \%(\mathrm{w} / \mathrm{v})$ MEA cultures of each fungal strain and incubating in the dark for 8 weeks. Mycelia and agar were then scraped from the surface of each block and blocks were combined in pairwise $2 \times 2$ block configurations (Section 2.5.2). In along-vessel treatments neighbouring blocks colonised by the same species ( $n=2$ per species) were arranged with wood vessels in parallel, pairs of blocks colonised by different species were placed cutend to cut-end. In across vessel treatments, neighbouring blocks colonised by the same species $(n=2$ per species) were arranged with cut ends adjoining and pairs of blocks colonised by different species were arranged with the wood vessels in parallel. Single species primary resource capture controls were comprised of four wood blocks colonised by E. glandulosa. Microcosms were each provided $10 \mu 1$ autoclaved distilled $\mathrm{H}_{2} \mathrm{O}$ at experimental set-up were not watered after this point and incubated at $15^{\circ} \mathrm{C}$ in the dark for 8 weeks following experimental set-up. All treatments were repeated in triplicate $(n=15)$. The experiment was run for 8 weeks which wood blocks were broken up into thirds for re-isolations 
(Section 2.5.3), GC-MS metabolome analysis (Section 2.6) and LC-MS/MS proteome analysis (Section 2.7).

\subsubsection{Data analyses}

\subsubsection{Untargeted GC-MS metabolomics}

GC-MS chromatogram features were pre-processed, deconvoluted, aligned and assigned putative identifications using AMDIS (D'Arcy \& Mallard 2004), SpectConnect (Flory et al. 2007) and the NIST 11 (Wiley) and GMD (Kopka et al. 2005) spectral databases (Section 2.6.3). Metabolites conserved across two out of three replicates in each treatment group were reported using SpectConnect.

All data analyses were undertaken in MetaboAnalyst 4.0 (Chong et al. 2018). To examine the degree to which metabolite identities and concentrations contributed to the separation of samples into their treatment groups, PLS-DA models were built at various levels: when samples were grouped based on the initial colonising species to consider primary versus secondary resource capture behaviours; when samples were split into two separate experiments considering a single vessel orientation; and the full experimental design. The overall differences in the metabolome of E. glandulosa following primary resource capture and replacement interactions was examined by combining the across- and along-vessel recapture samples by initial colonising species and applying T-tests with 5\% Benjamini-Hochberg FDR for multiple comparisons. To examine the changes in strategy employed by E. glandulosa following replacement of the two different early-arriving species, the along- and across-vessel treatments were considered independently as two separate experiments and one-way ANOVA with a 5\% BanjaminiHochberg FDR correction for multiple comparisons (Benjamini \& Hochberg 1995) and fisher's LSD posthoc tests were applied to both experiments. Finally, two-way ANOVA with a 5\% Benjamini-Hochberg false discovery rate (FDR) correction for multiple comparisons (Benjamini \& Hochberg 1995) and Fisher's LSD post hoc tests were applied to the full experiment to test for the influence of interactions between the two independent variables, initial coloniser identity and vessel orientation, on the metabolome of E. glandulosa.

To further examine the strategies employed by E. glandulosa in territory recapture from O. mucida and $S$. rugosum across vessels and along vessels, Metabolomics Pathway Analysis (MetPA) was carried out on pairwise groupings with reference to the fungal metabolome (Saccharomyces cerevisiae) in MetaboAnalyst 4.0 (Chong et al. 2018).

\subsubsection{Untargeted LC-MS/MS proteomics}

LC-MS/MS detected proteins (Section 2.7.1) were analysed using the predicted protein databases of $E$. glandulosa HHB12029 (Nagy et al. 2016) and O. mucida CBS55879 (Barrasa 2014) using MaxQuant (version 1.6.2.3) with integrated Andromeda for database searching (Cox \& Mann 2008; Section 2.7.2). Functional annotation and the mapping of annotated enzymes to KEGG pathways was undertaken in B2G (Section 
2.7.3). Functional annotations were explored by way of Directed Acyclic Graphs (DAGs) using B2G, categorised by biological process, molecular function and cellular component (Appendix IV). Sequence filtering during graph construction was undertaken using default settings. The proportion of proteins associated with nodes of the DAGs were examined by way of pie charts constructed using various levels of ontology (Appendix IX).

Proteins exhibiting differential expression between treatment groups were identified in B2G using Fisher's exact tests with 5\% Benjamini-Hochberg FDR correction for multiple comparisons (Benjamini \& Hochberg 1995) applied to pairwise treatment contrasts (Section 2.7.4).

\subsection{Results}

\subsubsection{Untargeted GC-MS metabolomics of primary and secondary resource capture by Exidia glandulosa}

SpectConnect identified a total of 740 conserved features following removal of internally-matching peaks and data pre- processing. Of these, 39 compounds were putatively identified and matched to metabolites (Appendix III) including 10 fatty acids, 8 organic acids, 8 sugar alcohols and 3 saccharides. Functional annotation identified 15 associated with carbohydrate metabolism, 9 with energy metabolism, 7 with lipid metabolism, 2 with secondary metabolite biosynthesis and one each with amino acid metabolism, xenobiotic biodegradation and metabolism and polyphenol degradation. Three compounds (Dipropylformamide, 1-Deoxypentitol and 8,2'-aminoanhydroadenosine) could not be classified or assigned to biological functions.

\subsubsection{Effect of primary and secondary resource capture on the metabolome of Exidia glandulosa}

Samples were separated into primary and secondary resource capture treatments by a PLS-DA model comprising three components $(\mathrm{R} 2=1.00 ; \mathrm{Q} 2=0.68$; Accuracy = 1.00; Fig. 5.1 and Appendix III) which explained $51.4 \%$ of the variance in the metabolome. The primary axis separated samples based on whether E. glandulosa underwent primary or secondary resource 


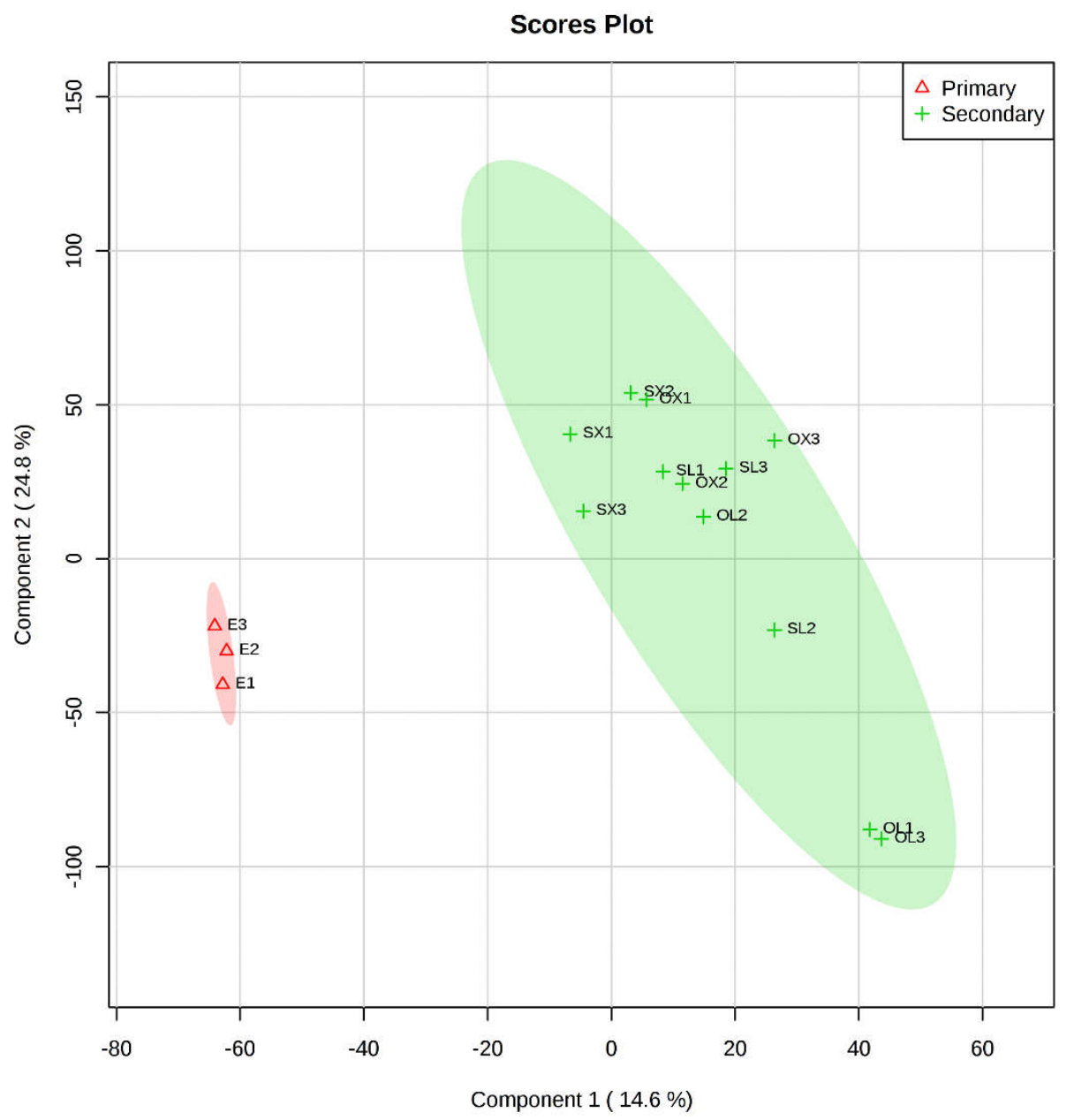

Figure 5.1 PLS-DA model of Exidia glandulosa metabolome following primary and secondary resource capture.

Samples were pooled into primary $(n=3$; red) and secondary $(n=12$; green) resource capture treatments irrespective of initial colonising species identity or wood vessel orientation. Shaded areas, $95 \%$ confidence intervals.

Table 5.1 T-tests of differences in compounds expressed by Exidia glandulosa following primary and secondary resource capture.

Samples were pooled into primary $(n=3$; red) and secondary $(n=12$; green) resource capture treatments irrespective of initial colonising species identity or wood vessel orientation.

\begin{tabular}{lcccc}
\hline Compound & t.stat & p.value & -LOG10(p) & FDR \\
\hline P.96 & 8.8443 & $7.33 \mathrm{E}-07$ & 6.1347 & 0.000543 \\
P.84 & 7.3112 & $5.91 \mathrm{E}-06$ & 5.2286 & 0.00184 \\
P.70 & 7.1515 & $7.46 \mathrm{E}-06$ & 5.1272 & 0.00184 \\
P.73 & 6.6391 & $1.61 \mathrm{E}-05$ & 4.7919 & 0.002988 \\
P.195 & 6.0107 & $4.37 \mathrm{E}-05$ & 4.3596 & 0.006466 \\
P.159 & 5.7394 & $6.83 \mathrm{E}-05$ & 4.1657 & 0.008422 \\
P.143 & 5.226 & 0.000164 & 3.7864 & 0.017287 \\
P.29 & 4.8461 & 0.000319 & 3.4957 & 0.02954 \\
\hline
\end{tabular}




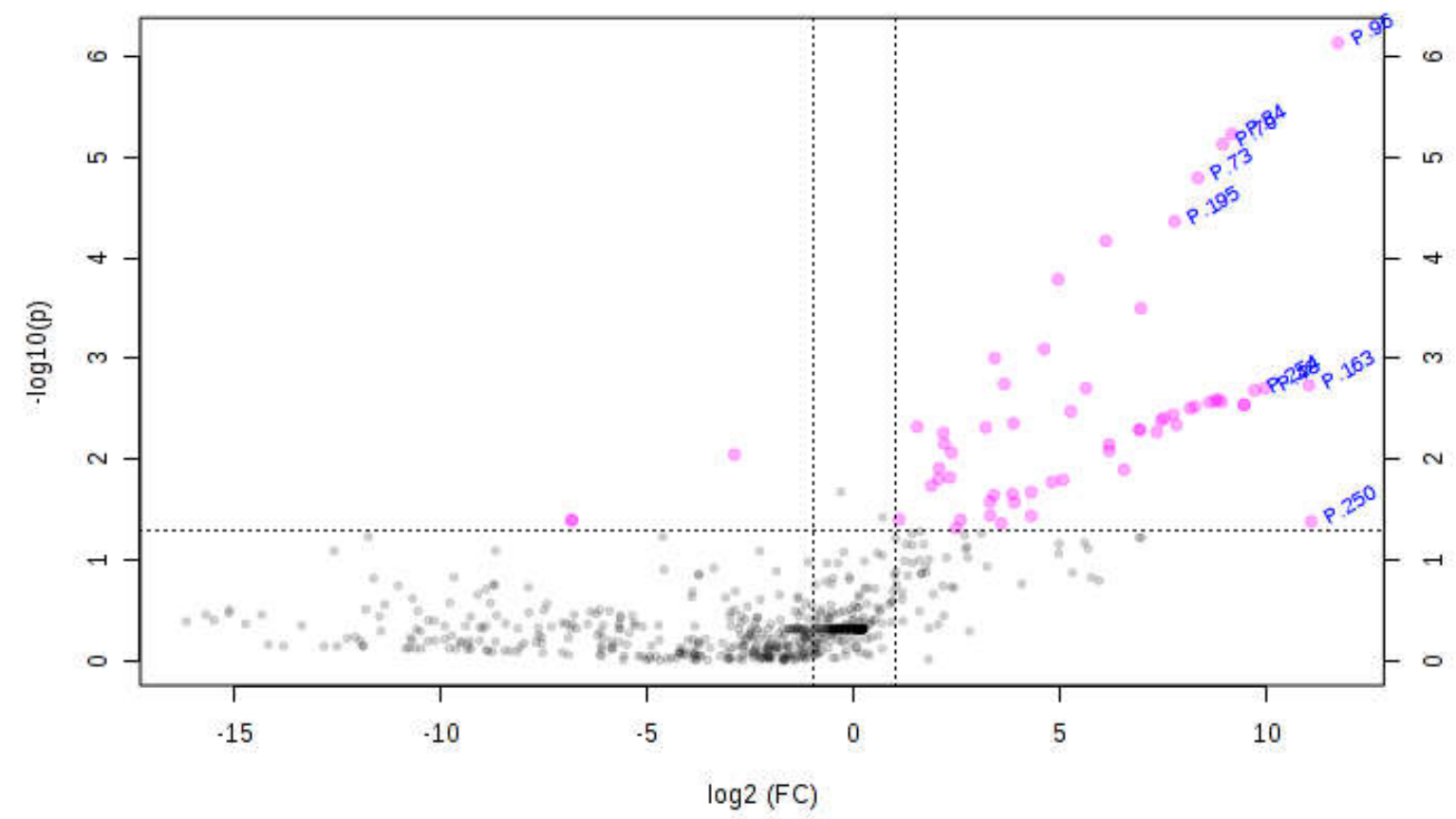

Figure 5.2 Volcano plot of Exidia glandulosa metabolome following secondary resource capture compared with primary resource capture.

Samples were pooled into primary $(n=3 ;)$ and secondary $(n=12)$ resource capture treatments irrespective of initial colonising species identity or wood vessel orientation. Pink, compounds exhibiting < -2.0 (left) or $>2.0$ (right) fold change in secondary resource capture samples; blue labels, compounds exhibiting significantly different concentrations (T-test; $\mathrm{P}<0.05$; FDR $=5 \%$ raw P-value).

capture and explained $14.6 \%$ of total variation in the metabolome. The metabolome was considerably more varied following secondary resource capture, indicating that further separation of samples out into treatment groups was appropriate. 180 compounds contributed to the separation of samples into groups including dipropyl-formamide, sebacic acid, D-xylitol, succinic acid, D- mannitol, 1-octanol, caprylic acid, glyoxylic acid and glycerophosphoric acid.

The concentration of 11 compounds differed following secondary resource capture compared with primary capture all of which were present in greater concentration in replacement treatments (T-tests; $\mathrm{P}$ $<0.001$; Table 5.1 and Fig. 5.2). All differentially expressed compounds were unidentified (P23, P70, P73, P84, P96, P143, P159 and P195).

\subsubsection{Effect of early-arriving species on the metabolome of Exidia glandulosa following replacement along the wood vessels}

Samples were separated into treatment groups along the first component of a PLS-DA model which explained $20.3 \%$ of variation in the metabolome however the $95 \%$ confidence intervals of secondary resource capture treatments initially colonised by O. mucida $(n=3)$ and S. rugosum $(n=3)$ overlapped indicating that the metabolome associated with these groups may not be statistically different from each other (Fig 5.3). The full model, comprising 3 components, explained $51.4 \%$ of total variation ( $2=1.00$; $\mathrm{Q} 2=0.45 ;$ Accuracy $=0.75 ;$ Appendix III). The 


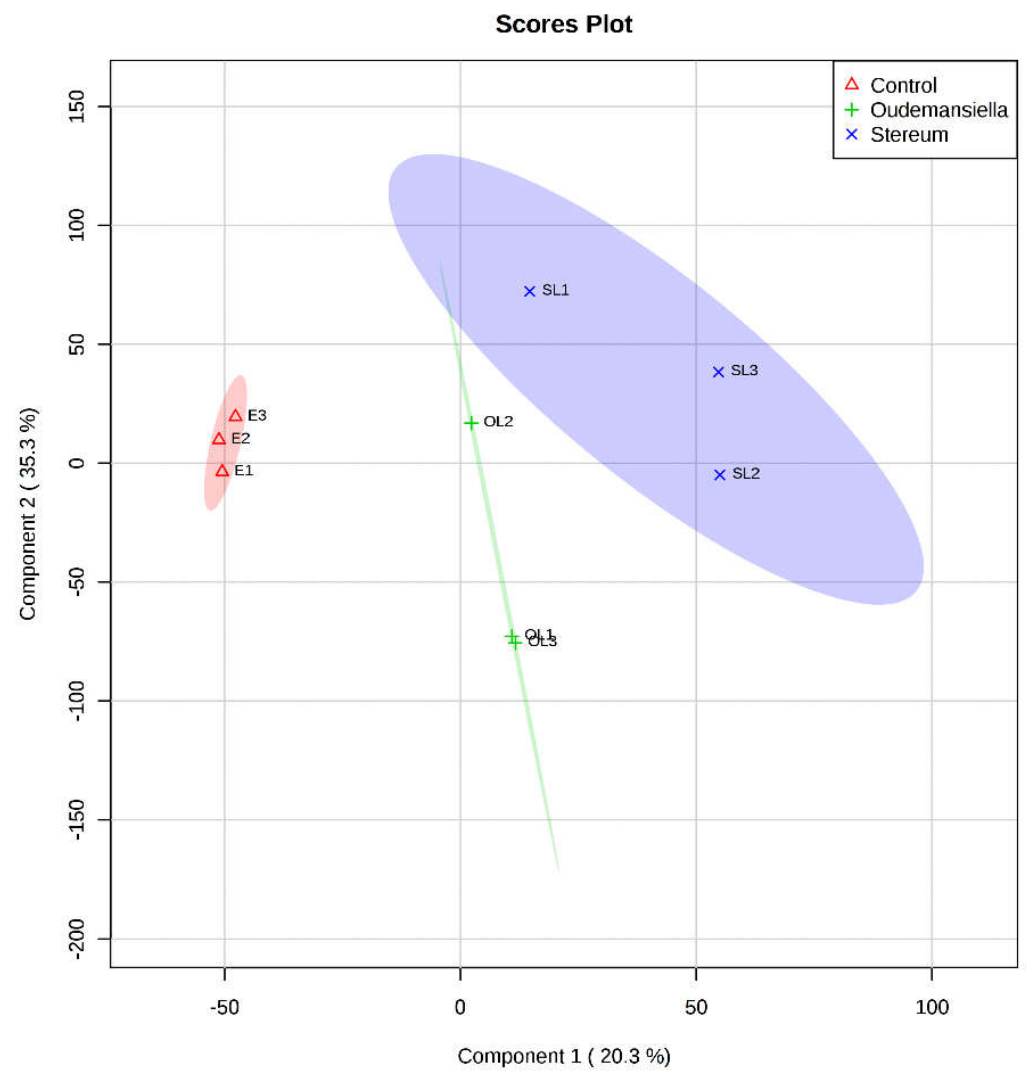

Figure 5.3 PLS-DA model of Exidia glandulosa metabolome following primary and secondary resource capture along xylem vessels.

Red, primary resource capture samples colonised by E. glandulosa alone; green, samples where wood had been captured from $S$. rugosum; blue, samples where wood had been captured from O. mucida. Shaded areas, 95\% confidence intervals.

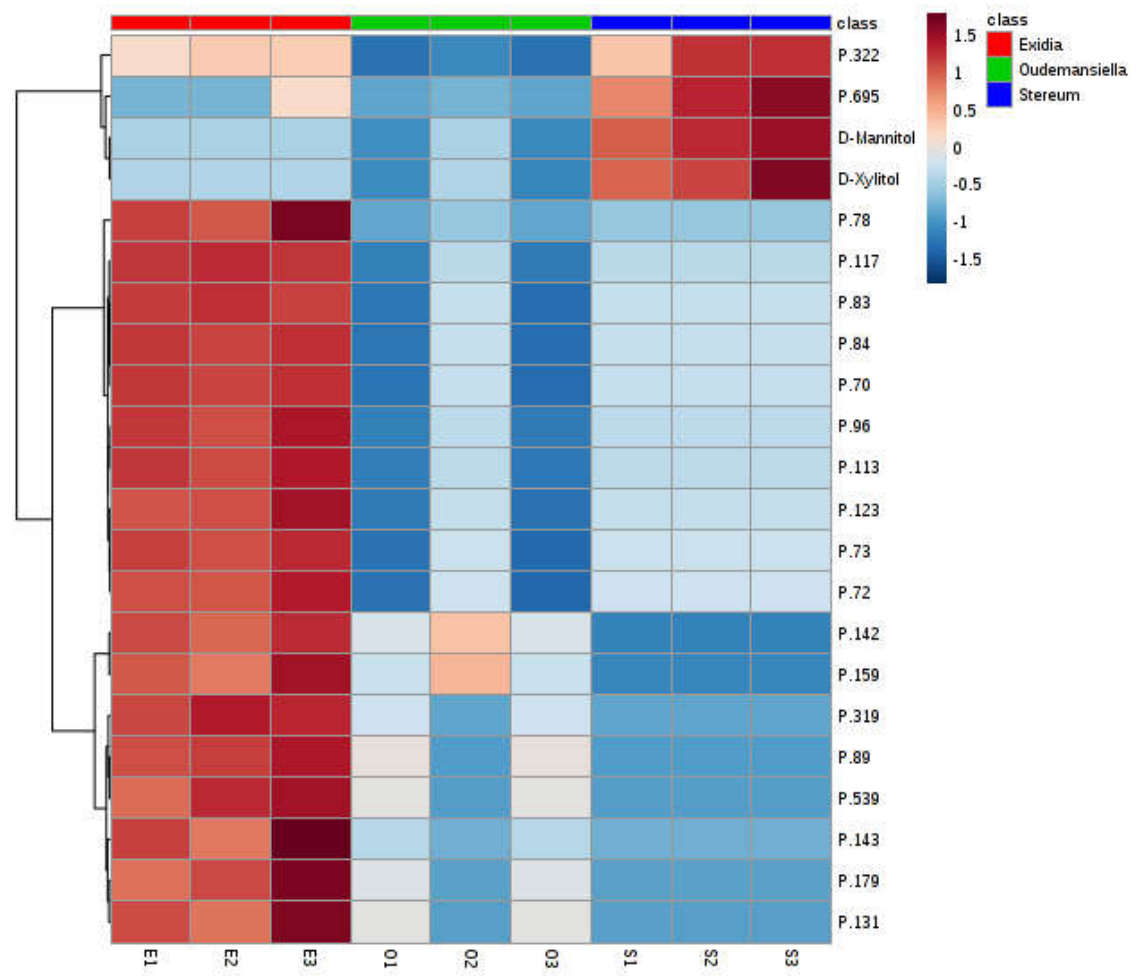

Figure 5.4 One-way ANOVA of the metabolome of Exidia glandulosa following primary resource capture and secondary resource capture from Oudemansiella mucida and Stereum rugosum along the wood vessels.

All compounds were detected in significantly different concentrations between the three treatment groups (ANOVA; P < 0.05 ; 5\% Benjamini-Hochberg FDR correction and Fisher's LSD post hoc tests) 
greatest variation was explained by differences in the concentration of D-mannitol and D-xylitol. The concentration of citric acid, glycerophosphoric acid-tetra-TMS, 1,2,4-butanetriol, 3-methylbenzoate, 1octanol, sebacic acid, malic acid, succinic acid, hydrogen sulphide and glyoxylic acid along with 174 unidentified compounds were also important in driving sample separation into treatment groups (VIP score > 1.0; Appendix III). The top three compounds (D-mannitol, D-xylitol and compound P349) had greater concentration following secondary capture compared with primary capture and also in wood captured from S. rugosum compared with O. mucida. The concentrations of 22 compounds were significantly different between the three treatment groups (one-way ANOVA; P $\leq 0.05$; Fig. 5.4 and Appendix III)

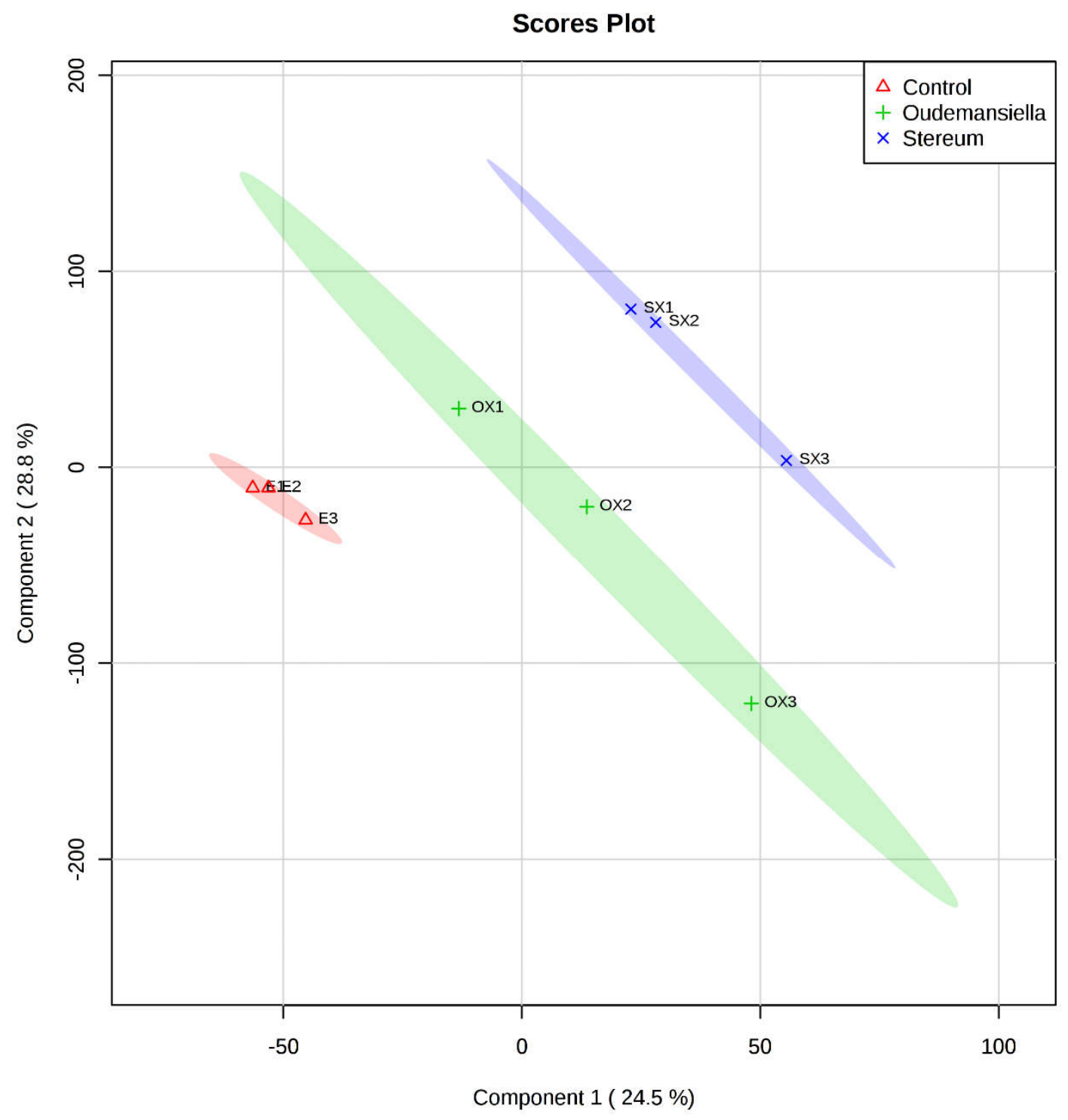

Figure 5.5 PLS-DA model of Exidia glandulosa metabolome following primary and secondary resource capture across xylem vessels.

Red, primary resource capture samples colonised by E. glandulosa alone; green, samples where wood had been captured from $S$. rugosum; blue, samples where wood had been captured from O. mucida. Shaded areas, 95\% confidence intervals. 


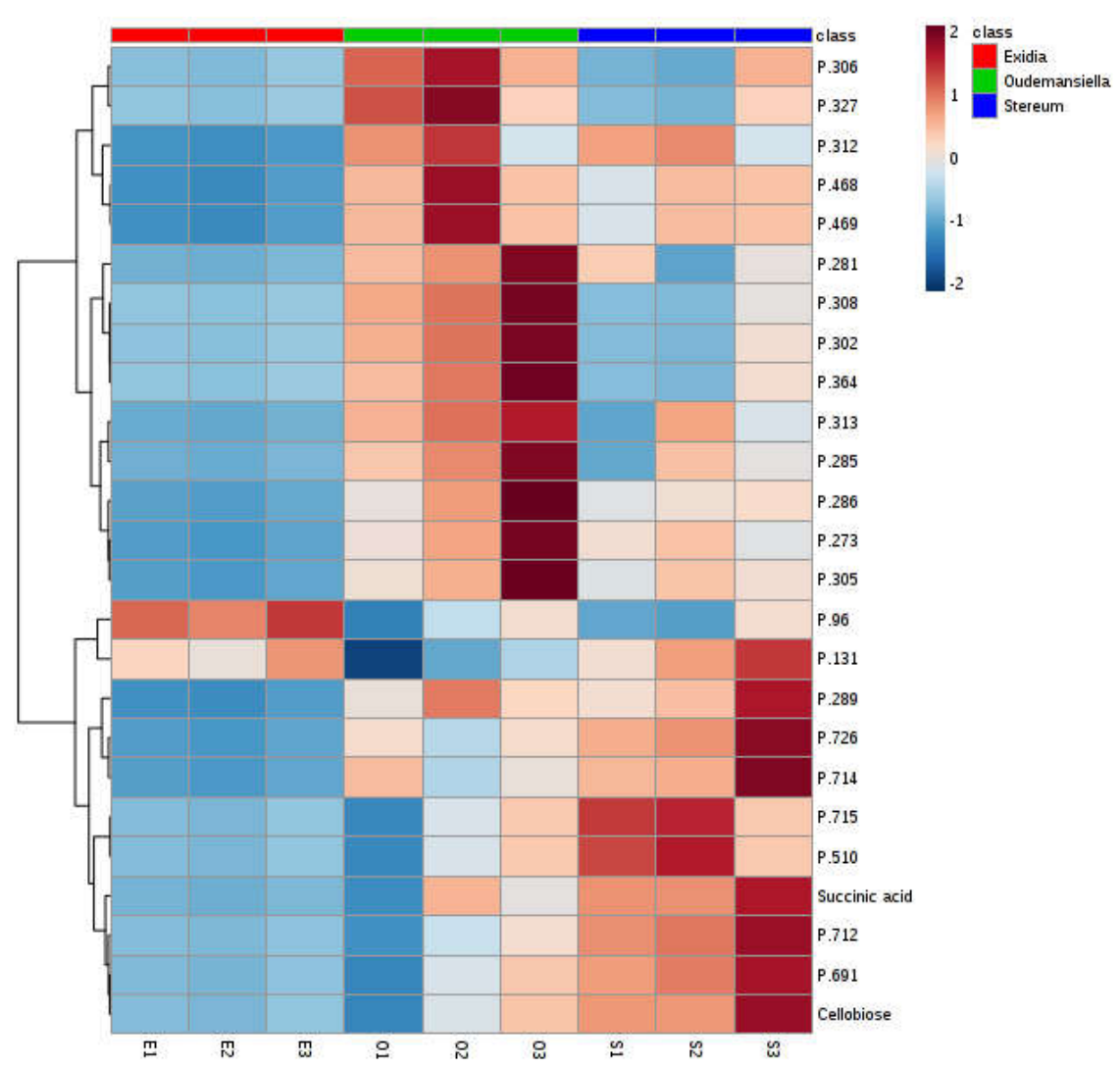

Figure 5.6 One-way ANOVA of the metabolome of Exidia glandulosa following primary resource capture and secondary resource capture from Oudemansiella mucida and Stereum rugosum along the wood vessels.

ANOVA was insignificant. Heatmap shows the relative concentration of the 25 compounds in the E. glandulosa metabolome that came closest to returning significant ANOVA results (ANOVA; P > 0.05; 5\% Benjamini-Hochberg FDR correction and Fisher's LSD post hoc tests).

\subsubsection{Effect of early-arriving species on the metabolome of Exidia glandulosa following replacement across the wood vessels}

Samples were separated into treatment groups by the first two components of a PLS-DA model, the first of which explained $24.5 \%$ variation in the metabolome of E. glandulosa and the second of which explained $28.8 \%$ variation (Fig. 5.5). The full model had 3 components and explained $60.9 \%$ variation in the metabolome $(\mathrm{R} 2=1.00 ; \mathrm{Q} 2=0.32$; Accuracy = 0.56; Appendix III). The concentrations of 145 compounds were important in driving the observed separation of samples into their treatment groups, succinic acid, which was the most important, cellobiose, glyoxylic acid, D-mannitol, D-xylitol, hydrogen sulphide, dipropylformamide and 2-oxo-glutaric acid. Of these, the top 5 compounds were observed to have higher concentration following replacement of $S$. rugosum and lower concentration primary capture controls as compared with wood captured from O. mucida (VIP score > 1.0; Appendix III). There were no significant differences (One-way ANOVA; P > 0.05) in the metabolome of E. glandulosa when territory was captured from either species across the xylem vessels (Fig. 5.6 and Appendix III). 


\subsubsection{Interactive effects of early-arriving species and wood anatomy on the metabolome of Exidia glandulosa}

Samples were separated into primary and secondary resource capture treatments by a PLS-DA model comprising three components $(\mathrm{R} 2=1.00 ; \mathrm{Q} 2=0.60$; Accuracy = 1.00; Appendix III) which explained $47.3 \%$ of the variance in the metabolome. The primary axis separated samples based on whether $E$. glandulosa underwent primary resource capture or recaptured resource from either O. mucida or S. rugosum and explained $9.2 \%$ of variation in the metabolome. The metabolome of E. glandulosa in wood captured from O. mucida was more varied than in wood captured from S. rugosum and wood wherein E. glandulosa had undergone primary resource capture only the metabolome was noticeably more similar than in either secondary resource capture treatments and there was no apparent separation between wood captured along and across vessels following replacement of either initial colonising species (Fig. 5.7). The relative concentrations D-mannitol and D-xylitol were the most important compounds driving separation of samples into their treatment groups. Succinic acid, 1,2,4-butanetriol, glycerophosphoric acid, cellobiose, citric acid, glyoxylic acid, hydrogen sulphide, 3-methylbenzoate, 1-octanol, sebacic acid and 173 unidientified compounds also made substantial contributions to sample separation into their respective treatment groups (VIP score > 1.0; Appendix III).

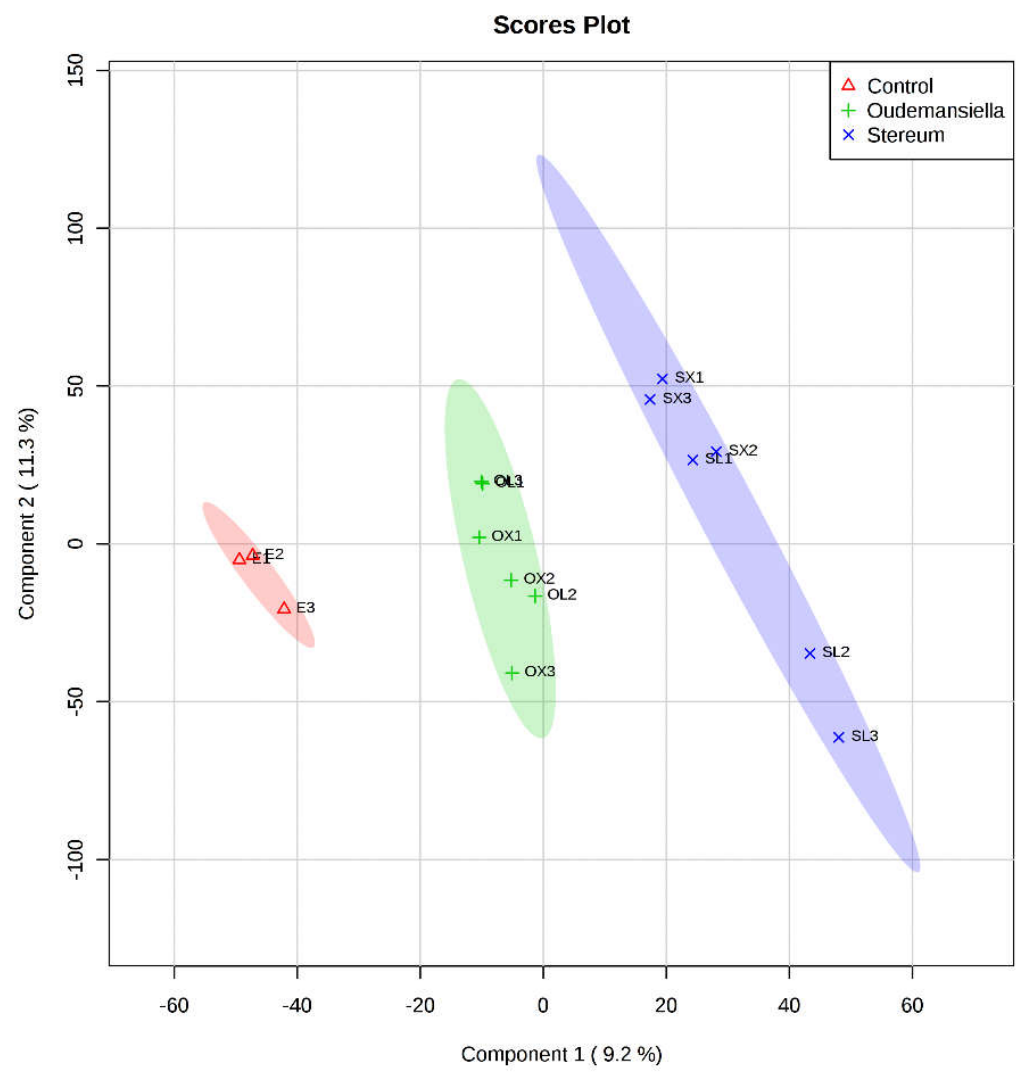

Figure 5.7 Interactive effect of initial colonising species and vessel orientation on the metabolome of Exidia glandulosa. Red, primary resource capture samples colonised by E. glandulosa alone; green, samples where wood had been captured from $S$. rugosum; blue, samples where wood had been captured from O. mucida. Shaded areas, 95\% confidence intervals. 


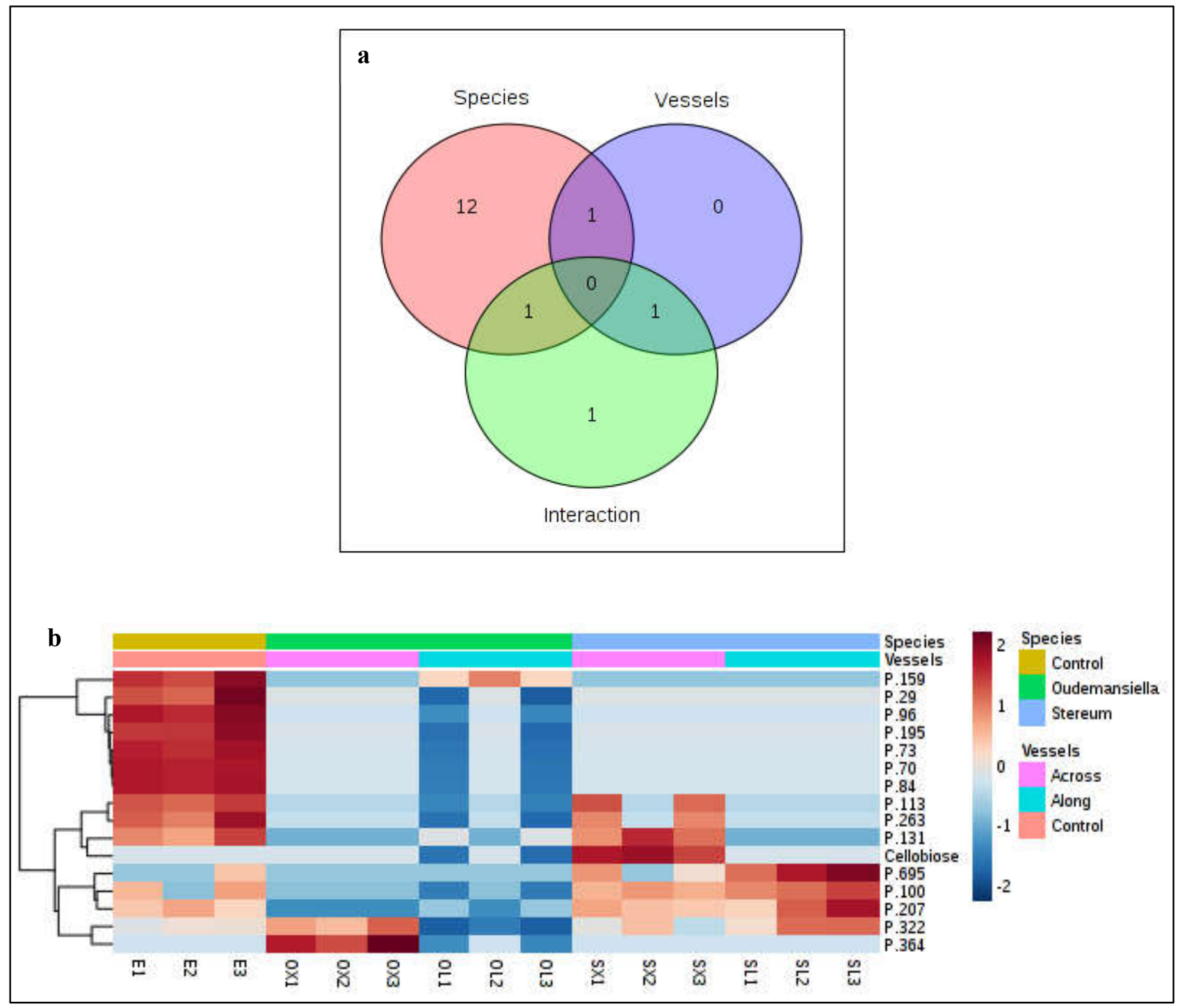

Figure 5.8 Interactive effects of early-arriving species and wood vessel orientation on the metabolome of Exidia glandulosa assessed by two-way between samples ANOVA.

(a) Venn-diagram of two-way ANOVA results; (b) heat map of significant two-way ANOVA results (P $<0.05 ; 5 \%$ BenjaminiHochberg FDR correction and Fisher's LSD post hoc tests).

The abundances of 12 compounds were significantly affected by the identity of the initial colonising species: E. glandulosa (primary resource capture), O. mucida or S. rugosum (two-way ANOVA; P $\leq 0.05$; Fig. 5.8 and Appendix III). The abundances of a further four compounds were significantly affected $(\mathrm{P} \leq$ $0.05)$ by the interaction between initial colonising species and vessel orientation. Vessel orientation alone did not exert any significant effects on compound abundances. Of these 16 compounds only one, cellobiose, was putatively identified and this metabolite was up-regulated in wood recaptured from $S$. rugosum across the vessels and down-regulated in all other groups. The concentrations of compounds 29, $96,195,73,70$ and 84 were increased only in wood undergoing primary resource capture by $E$. glandulosa. Compound 159 was present in greater concentration in controls and in wood recaptured from O. mucida along the vessels. Concentration of compounds 113, 263 and 131 was elevated in controls and wood recaptured from S. rugosum across the vessels while that of compounds 695, 100 and 207 was lower in controls and all wood recaptured from S. rugosum. Concentration of compound 322 was 
elevated in wood recaptured from O. mucida across the vessels and all wood recaptured from S. rugosum and that of compound 364 was elevated only in wood recaptured from O. mucida across the vessels.

\subsubsection{Pathway enrichment analysis of Exidia glandulosa metabolome following primary and secondary resource capture}

There were significant changes in the activities of pathways between treatment groups suggesting a change in strategy employed by E. glandulosa driven by both the identity of the initially colonising species and the direction of the interaction relative to xylem vessels (MetPA Enrichment Analysis; pairwise treatment contrasts; $\mathrm{P} \leq 0.05$; Table 5.2). In across vessel treatments, there was a switch from arginine and proline biosynthesis (amino acid metabolism) to the TCA cycle (energy metabolism) and glyoxylate and dicarboxylate metabolism (carbohydrate metabolism) being enriched when territory recapture was compared with controls and between original colonising species respectively. In alongvessel treatments, inositol phosphate metabolism (carbohydrate metabolism), sulfur metabolism (energy metabolism) and alanine, aspartate and glutamate metabolism (amino acid metabolism) pathways were enriched in territory recapture groups as compared with controls.

When comparing S. rugosum to O. mucida recapture groups there was a switch to pyruvate metabolism (aarbohydrate metabolism), glyoxalate and dicarboxylate metabolism (carbohydrate metabolism), glycine, serine and threonine metabolism, valine, leucine and isoleucine metabolism, cysteine and methionine metabolism (amino acid metabolism) and pantothenate and CoA biosynthesis (metabolism of cofactors and vitamins) pathways being enriched in S. rugosum replacement treatments. 
y

卷罟考

छ ज ज्ञ

है दू

तี

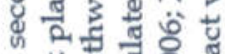

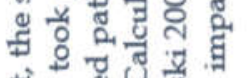

穴边

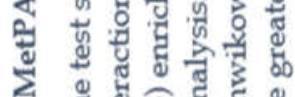

ङ झ

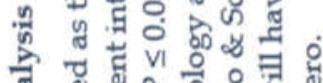

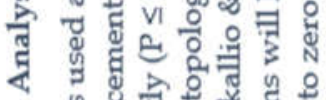

त

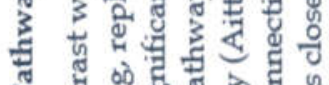

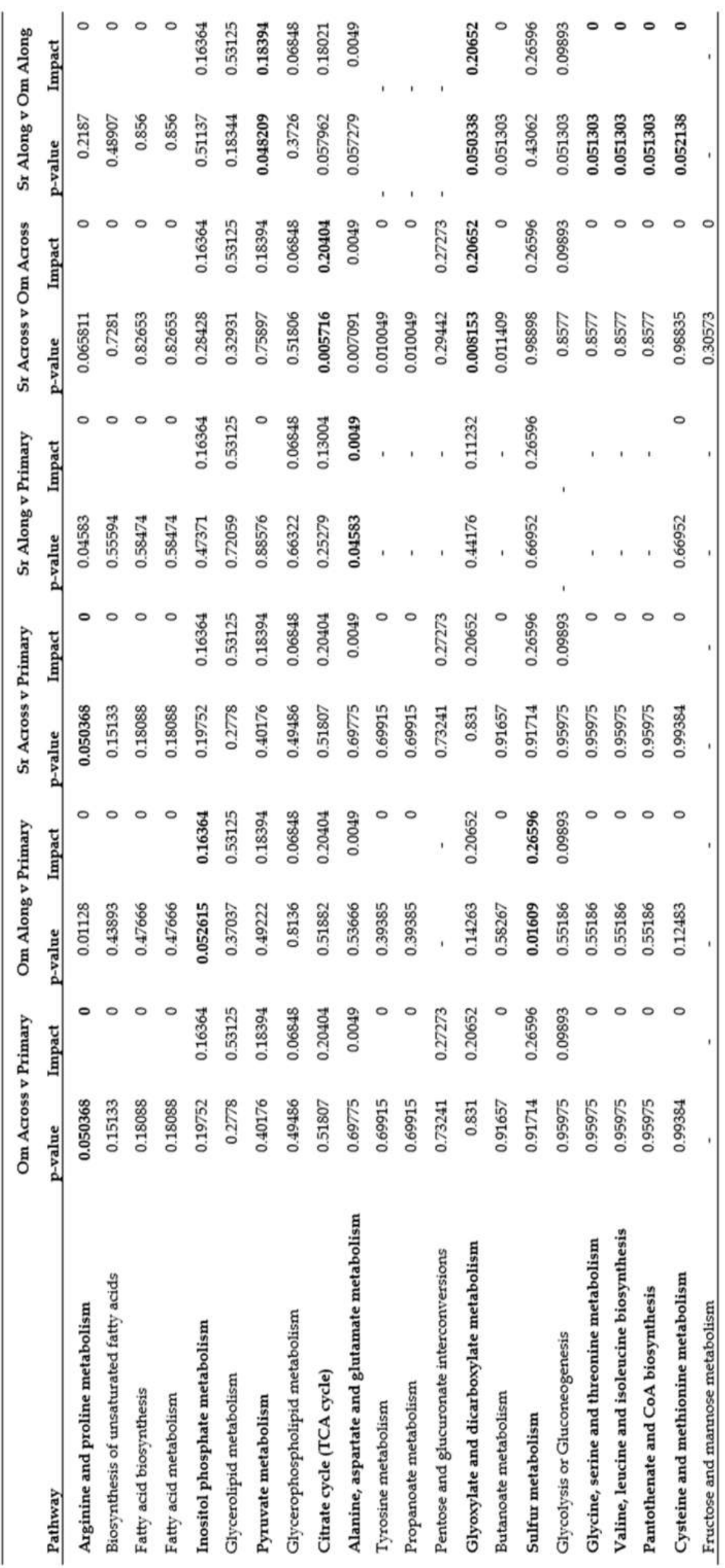

Page | 85 


\subsubsection{Functional direct shotgun LC-MS/MS proteomics of the proteome of Exidia glandulosa following primary and secondary resource capture}

MaxQuant identified the sequences of 135 proteins associated with the full experiment following alignment with E. glandulosa HHB12029 (Nagy et al. 2016) and O. mucida CBS55879 (Barrasa 2014) proteomes (Appendix IV). Of these 128 successfully completed the Gene Ontology (GO) pipeline including GO slimming.

Exploration of functional classifications indicated a potential change in strategy in E. glandulosa following secondary resource capture compared with primary resource capture (Appendix IV). When classified by biological process E. glandulosa expressed a slightly more varied proteome following primary resource capture then following secondary resource capture. While there was little difference in the relative contribution major processes (cellular protein metabolism, gene expression, macromolecule biosynthesis, organonitrogen compound biosynthesis, peptide metabolism, cellular amide metabolism, and cellular macromolecule biosynthesis), under primary resource capture E. glandulosa uniquely expressed proteins associated with catabolism of nucleobase-containing compounds, heterocycle catabolism, catabolism of organic cycle compounds, catabolism of aromatic compounds, catabolism of cellular nitrogen compounds, protein modification and macromolecule modification. Following secondary resource capture, E. glandulosa did not express any proteins associated with unique biological processes. When classified by molecular function, under primary resource capture E. glandulosa uniquely expressed proteins associated with protein binding.

In total 66 enzymes were expressed in the experiment (Fig. 5.9). The majority of enzymes $(n=23)$ were hydrolases which included those acting on acid anhydrydes $(n=15)$, on peptide bonds $(n=7)$ and on ether bonds $(n=1)$. Oxidoreductases $(n=16)$ and transferases $(n=14)$ were also well represented. Of the 66 enzymes present in the study, 53 were associated with primary resource capture and 66 with secondary resource capture in E. glandulosa. Enzymes followed similar distributions under both conditions, with the majority of enzymes belonging to the hydrolases and with oxidoreductases and transferases also being well represented. Similar pathways were utilised by E. glandulosa when capturing resource as a primary colonist and following replacement interactions. In both scenarios there was evidence that a considerable proportion of the E. glandulosa proteome in wood is dedicated to the biosynthesis and metabolism of antibiotics. 

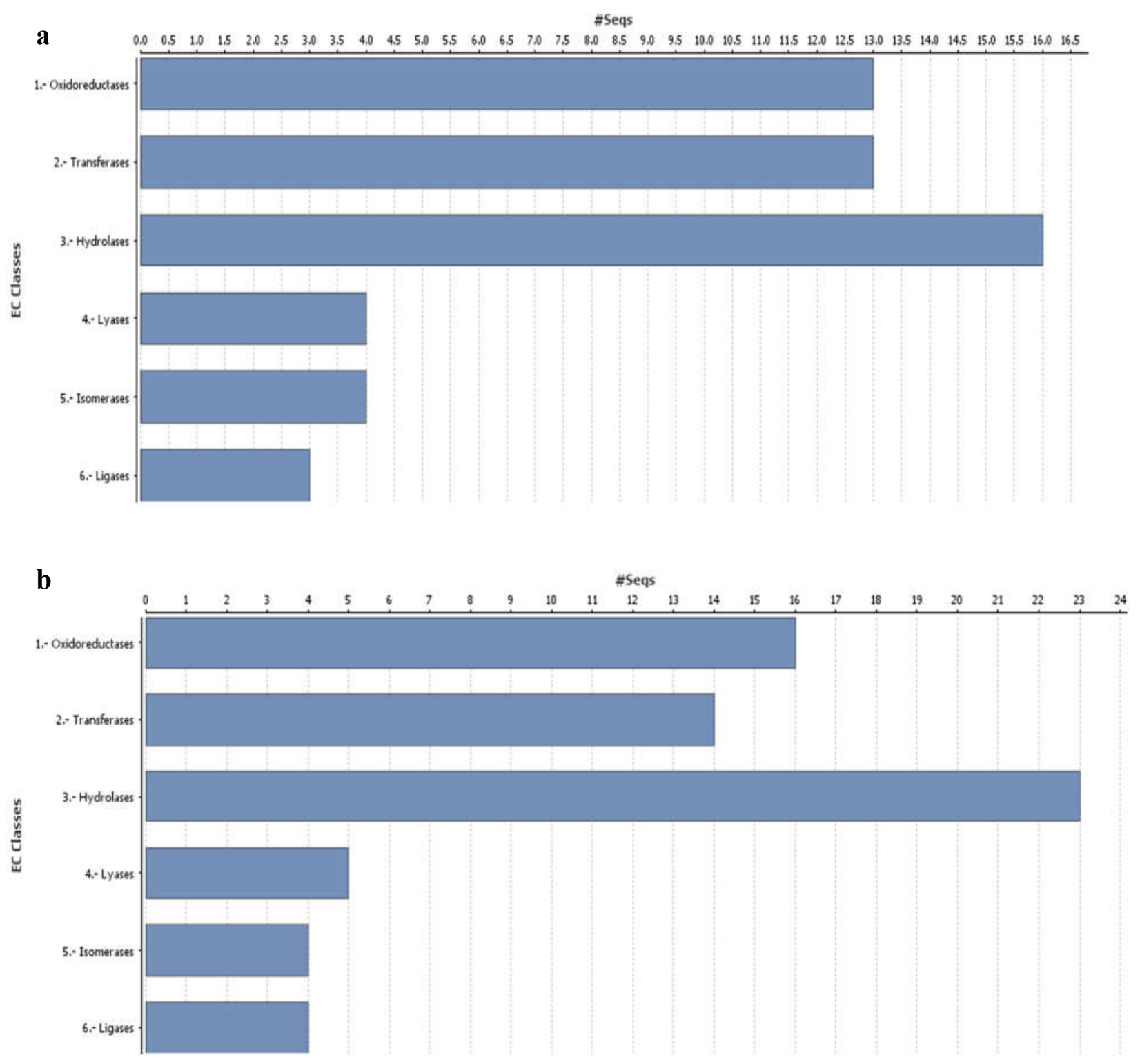

Figure 5.9 Enzyme commission codes mapped to the proteome of Exidia glandulosa.

(a) following primary resource capture; (b) following secondary resource capture 
Biosynthesis of antibiotics

Glycolysis / Gluconeogenesis

Pyruvate metabolism

Citrate cycle (TCA cycle)

Purine metabolism

Pentose phosphate pathway

Glyoxylate and dicarboxylate metabolism

Cysteine and methionine metabolism

Carbon fixation

Amino sugar and nucleotide sugar...

Valine, leucine and isoleucine degradation

Starch and sucrose metabolism

Methane metabolism

Tryptophan metabolism

Selenocompound metabolism

Pentose and glucuronate interconversions

Glycerolipid metabolism

Glutathione metabolism

Galactose metabolism

Fructose and mannose metabolism

Ascorbate and aldarate metabolism

Thiamine metabolism

Synthesis and degradation of ketone bodies

Pyrimidine metabolism

Propanoate metabolism

Phenylpropanoid biosynthesis

Pantothenate and CoA biosynthesis

Lysine degradation

Limonene and pinene degradation

Insect hormone biosynthesis

Histidine metabolism

Fatty acid degradation

Drug metabolism - other enzymes

Chloroalkane and chloroalkene degradation

Butanoate metabolism

beta-Alanine metabolism

Arginine biosynthesis

Arginine and proline metabolism

Alanine, aspartate and glutamate..

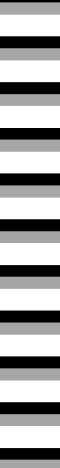

$-$

$\square$

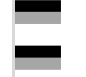

-
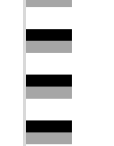

$$
\text { - }
$$

- Primary Secondary

Figure 5.10 KEGG pathway analysis of the proteomoe of Exidia glandulosa following primary and secondary resource capture. 


\subsubsection{Differential protein expression of Exidia glandulosa following secondary resource capture}

Significant differences in E. glandulosa's expression profile were identified under all pairwise treatment contrasts (Fisher's exact tests; $\mathrm{P} \leq 0.05$; Table 5.2). When E. glandulosa recaptured wood from O. mucida in both along- and across-vessel orientations the majority of differentially expressed proteins were downregulated compared with primary resource capture controls. In contrast, when E. glandulosa recaptured territory from S. rugosum the majority of differentially expressed proteins were detected in greater abundance compared with both primary resource capture controls and secondary resource capture from O. mucida, including several ribosomal and proteasomal proteins. Furthermore, there were a greater number of differentially expressed proteins, both up- and down-regulated, in all treatment groups where territory recapture was undertaken across vessels compared with the comparable treatment in along-vessel territory recapture. Four genes encoding NAD-malate dehydrogenase were differentially expressed in the proteome of E. glandulosa, with their associated proteins being present in lower abundance following capture of wood from O. mucida in both along $(n=2)$ and across-vessel $(n=2)$ treatments. Following capture of wood from S. rugosum, heat shock cognate 70 was down-regulated in both vessel orientation treatments. Various GTPases were up-regulated in treatments where E. glandulosa recaptured territory from $S$. rugosum compared with both primary resource capture controls and with secondary resource capture from $O$. mucida.

Table 5.3 Fisher's exact tests undertaken on pairwise treatment contrasts of Exidula glandulosa proteome following primary and secondary resource capture.

Pairwise treatment contrasts where the first-stated treatment was used as the test set and the second-stated treatment was used as the contrast set (v, versus); Abund., indicates whether protein abundance was increased (up) or decreased (down) in the test set; O, Oudemansiella mucida; E, Exidia glandulosa; L, replacement took place along the wood vessels; X, replacement took place across the wood vessels.

\begin{tabular}{|c|c|c|c|c|}
\hline Treatment & Abund. & Gene & Protein & $\begin{array}{c}\text { Fold } \\
\text { Change }\end{array}$ \\
\hline \multirow{2}{*}{$\begin{array}{c}\text { OL v } \\
\text { Control }\end{array}$} & \multirow{2}{*}{ Down } & jgi|Oudmuc1|1542081 & NAD-malate dehydrogenase & $-7.7 \mathrm{E}+08$ \\
\hline & & jgi|Exigl1| 741889 & NAD-malate dehydrogenase & $-1.9 \mathrm{E}+08$ \\
\hline \multirow{4}{*}{$\begin{array}{c}\text { SL v } \\
\text { Control }\end{array}$} & Down & jgi|Exigl1 | 741561 & heat shock cognate 70 & -27.5 \\
\hline & \multirow{3}{*}{ Up } & jgi|Oudmuc1|1567216 & predicted protein - Armso draft_951744 & 27.7 \\
\hline & & jgi|Oudmuc1 | 408590 & $\begin{array}{l}\text { mitochondrial NAD-dependent isocitrate } \\
\text { dehydrogenase subunit } 1 \text { precursor }\end{array}$ & 26.8 \\
\hline & & jgi|Oudmuc1|1299829 & 40S ribosomal protein $\mathrm{S} 14$ & 26.2 \\
\hline \multirow{6}{*}{ SL v OL } & \multirow{6}{*}{ Up } & jgi|Oudmuc1|1277481 & Unidentified protein & 27.6 \\
\hline & & jgi|Oudmuc1|1267090 & small GTPase-binding protein & 26.5 \\
\hline & & jgi|Oudmuc1|1299829 & $\begin{array}{l}\text { mitochondrial NAD-dependent isocitrate } \\
\text { dehydrogenase subunit } 1 \text { precursor }\end{array}$ & 26.7 \\
\hline & & jgi|Oudmuc1 | 408590 & ribosomal protein S13 & 28.3 \\
\hline & & jgi|Exigl1 | 742271 & $\begin{array}{l}\text { PF00227_Proteasome subunit; } \\
\text { PF10584_Proteasome subunit A N- } \\
\text { terminal signature }\end{array}$ & 27.2 \\
\hline & & jgi|Oudmuc1|1567216 & rab small monomeric GTPase & 26.5 \\
\hline
\end{tabular}




\begin{tabular}{|c|c|c|c|c|}
\hline Treatment & Abund. & Gene & Protein & $\begin{array}{c}\text { Fold } \\
\text { Change }\end{array}$ \\
\hline \multirow{7}{*}{$\begin{array}{l}\text { OX v } \\
\text { Control }\end{array}$} & \multirow{5}{*}{ Down } & jgi|Oudmuc1|1251812 & copper radical oxidase & -29.7 \\
\hline & & jgi|Oudmuc1|1226069 & aldehyde dehydrogenase & -29.1 \\
\hline & & jgi|Oudmuc1 |1418197 & cyclophilin & -30.3 \\
\hline & & jgi|Oudmuc1|1542081 & NAD-malate dehydrogenase & -29.6 \\
\hline & & jgi|Exigl1 |741889 & NAD-malate dehydrogenase & -27.6 \\
\hline & \multirow{2}{*}{ Up } & jgi|Exigl1 | 663183 & $60 \mathrm{~S}$ ribosomal protein $\mathrm{L} 3$ & 28.0 \\
\hline & & jgi|Exigl1 | 758521 & $60 S$ ribosomal protein L5 & 27.3 \\
\hline \multirow{15}{*}{$\begin{array}{l}\text { SX v } \\
\text { Control }\end{array}$} & \multirow[t]{2}{*}{ Down } & jgi|Oudmuc1 & $\begin{array}{l}\text { manganese peroxidase isozyme } \\
\text { precursor }\end{array}$ & -29.1 \\
\hline & & jgi|Exigl1 | 741561 & heat shock cognate 70 & -27.6 \\
\hline & \multirow{13}{*}{ Up } & jgi|Oudmuc1|1285172 & rab GTPase activator & 27.2 \\
\hline & & jgi|Oudmuc1 | 686559 & PF01423_LSM domain & 25.6 \\
\hline & & jgi|Exigl1 | 647872 & glycosyltransferase family 35 protein & 26.1 \\
\hline & & jgi|Oudmuc1|1548312 & ubiquitin carboxyl-terminal hydrolase 5 & 26.0 \\
\hline & & jgi|Oudmuc1 | 1267120 & septin & 26.0 \\
\hline & & jgi|Oudmuc1 | 1430567 & Non-histone chromosomal protein 6 & 26.0 \\
\hline & & jgi |Oudmuc1 | 1221544 & ribosomal protein L2 & 27.5 \\
\hline & & jgi|Oudmuc1|346488 & 40s ribosomal protein s4 & 26.1 \\
\hline & & jgi|Oudmuc1|1248743 & ribosomal protein S5 & 28.4 \\
\hline & & jgi|Oudmuc1 | 408590 & $\begin{array}{l}\text { mitochondrial NAD-dependent isocitrate } \\
\text { dehydrogenase subunit } 1 \text { precursor }\end{array}$ & 26.4 \\
\hline & & jgi|Exigl1 | 758521 & $60 S$ ribosomal protein L5 & 27.6 \\
\hline & & jgi|Oudmuc1|1211663 & $40 S$ ribosomal protein S20 & 27.8 \\
\hline & & jgi|Exigl1 |695180 & cytoplasmic protein & 26.1 \\
\hline \multirow{9}{*}{ SX v OX } & Down & jgi|Oudmuc1 | 1552981 & aldehyde dehydrogenase & -28.1 \\
\hline & \multirow{8}{*}{ Up } & jgi|Oudmuc1|1221544 & ribosomal protein L2 & 27.5 \\
\hline & & jgi| Exigl1 | 676321 & 14-3-3 protein & 27.0 \\
\hline & & jgi|Oudmuc1|1229426 & GTP binding protein & 27.1 \\
\hline & & jgi|Exigl1 | 695180 & cytoplasmic protein & 26.1 \\
\hline & & jgi|Oudmuc1|1226069 & aldehyde dehydrogenase & 29.7 \\
\hline & & jgi| Exigl1 | 831331 & ATP-citrate synthase & 26.6 \\
\hline & & jgi|Oudmuc1|1418197 & cyclophilin & 30.3 \\
\hline & & jgi|Oudmuc1|1285172 & rab GTPase activator & 27.2 \\
\hline
\end{tabular}

\subsection{Discussion}

The identity of the earliest-arriving species exerted differential effects on the metabolism of a laterarriving pioneer species, E. glandulosa, at both the metabolite and protein level. At the protein level there was a clear, additive effect of early-arriving species identity and structural complexity of wood anatomy in relation to the orientation of replacement interactions. At the metabolite level, the presence of additive effects were less clear and early-arriving species identity exerted a stronger and clearer effect on the metabolome of E. glandulosa than wood vessel orientation. These data represent the very first study of Page | 90 
the impact of species arrival order and the resultant priority effect on the metabolism of any species in any ecological system.

Both the metabolome and proteome of the late-arriving species, E. glandulosa, differed between treatments when samples originally colonised by $O$. mucida or S. rugosum were considered irrespective of interaction orientation. Overall protein and metabolite expression levels differed according to the identity of the initial colonist. In samples where $O$. mucida was the initial colonising species, the majority of differentially expressed proteins were down-regulated compared with primary capture controls while the opposite was true in the metabolome. In particular genes expressing NAD-malate dehydrogenase, which plays a central role in numerous pathways linked to energy metabolism as well as facilitating the transport of intracellular metabolites (Musrati et al. 1998), along with aldehyde dehydrogenase, copper radical oxidase and a cyclophilin were present in lower abundance in wood captured from O. mucida compared with primary resource capture controls. Copper radical oxidase is implicated in lignin degradation and detoxification (Moody et al. 2018a) indicating that E. glandulosa may have invested more highly in lignocellulose decomposition during primary resource capture than following interactions with $O$. mucida. In contrast, in samples colonised initially by S. rugosum most differentially expressed proteins were present in greater abundance than in either wood captured from O. mucida or wood undergoing primary resource capture. It was particularly striking that a number of ribosomal (40S and 60S) proteins along with a proteasome subunit protein were present in greater abundance often following capture of resource from S. rugosum. This could relate to a slower growth rate of E. glandulosa following these replacement interactions with a switch in emphasis from nutrient acquisition to protein turnover and degradation (Bhattacharyya et al. 2014; Mathis et al. 2017). Following replacement of competitors ligninolytic fungi recycle the cellular components of their competitors as well as those of their own mycelia (Lindahl \& Finlay 2006). E. glandulosa's capability to recycle the constituents of $S$. rugosum mycelia which could result in an increase in the presence of proteins associated with protein degradation either because greater access to mycelial-bound nutrients is afforded or because it takes E. glandulosa a longer time to break down the foreigner's hyphae and access the nutrients present. Replacement interactions between E. glandulosa may have taken longer to complete than those involving O. mucida and so in the latter case the recycling of the initial colonist's mycelia may have been complete with E. glandulosa reverting to investment in acquiring nutrients from the wood matrix to a near-primary resource capture extent. Alternatively, given that $O$. mucida produces pseudosclerotal plates which are thick aggregations of mycelia that physically impede invasive mycelia (Boddy et al. 2017), it is possible that E. glandulosa was less capable of degrading the hyphae of the previous colonist in these treatments.

Replacement of S. rugosum had an opposite effect on the metabolome as on the proteome, with the majority of differentially expressed compounds following replacement of S. rugosum being down- 
regulated compared with controls. Of note was the greater abundance of proteins associated with proteolysis (particularly a mitochondrial NAD-dependent isocitrate dehydrogenase subunit 1 precursor and a RAB GTPase activator) and with the binding and hydrolysis of guanosine triphosphate (GTP; small GTPase-binding protein and RAB small monomeric GTPase) in wood captured from S. rugosum, indicating that protein turnover and signalling may be increased following the replacement of this species compared with replacement of $O$. mucida and with primary resource capture. It has been suggested that in wood decay fungi, intracellular proteolysis and the resultant mobilisation of protein stores is likely linked to a requirement for nitrogen, particularly due to the high $\mathrm{C}: \mathrm{N}$ ratio within wood (Wadekar et al. 2018). The increased abundance of proteins associated with proteolysis here could be indicative of E. glandulosa responding to differential decomposition of the substrate, including rates of decay, by the initially colonising species. The stronger long-term effect of S. rugosum on the proteome of E. glandulosa compared with that exerted by O. mucida is particularly striking given that O. mucida is known to have a highly defensive lifestyle and produce strong antifungal compounds. Unidentified compounds found to be present in greater concentration following replacement of the two initial colonising species may play roles in degrading the products of specialised metabolism, including oudemansin-A and other antifungal compounds, produced by the two early-arriving species.

These observations have important implications for fungal decay ecology given that later-stage decay fungi such as Trametes versicolor and Hypholoma fasiculare are routinely fed uncolonized wood to gauge their reactions to biotic and abiotic stimuli (A'Bear et al. 2014a; Hiscox et al. 2016a, b, 2017, 2018). Laterstage species rarely encounter fresh, uncolonized wood except in the presence of healthy, felled wood. Thus 'priming' the wood with a pioneer species may produce differential experiment outcomes which may be closer to those that drive ecosystem processes in nature. While it is not known how quickly $E$. glandulosa replaced the two original colonisers in the experiment, their complete replacement was demonstrated by pure E. glandulosa cultures being grown from wood chip re-isolations at 8 weeks following experiment set-up. Previous work suggests that replacement interactions between species associated with the primary and early secondary successions in wood blocks are often complete 3 to 6 weeks after experiment start (Hiscox et al. 2015b). These results are indicative therefore of relatively long-term impacts on the proteome and metabolome of the later-arriving species following the completion of replacement interactions, and this may have important implications in understanding the underlying molecular mechanisms of priority effects.

At different levels of fungal biochemistry, structural complexity of the substrate exerted different effects. Greater structural complexity led to a greater number of proteins being expressed with both lower and higher abundance, but effects were less clear at the metabolite level. Fewer proteins were differentially expressed when E. glandulosa replaced the initial colonists along the wood vessels compared with acrossvessel replacement. In addition, the greater effect S. rugosum seemingly exerted on the proteome was 
maintained following interactions in both orientations (i.e. when comparing S. rugosum across- or alongvessel replacement treatments with $O$. mucida counterparts). In across-vessel replacements a glycosyltransferase family 35 CAZyme and a cyclophilin, related to stress response, were among those present in greater abundance. However a cyclophilin and the CAZyme copper radical oxidase were also present with greater abundance in control treatments compared with wood captured from O. mucida across the wood vessels. Across-vessel replacement clearly had a greater impact on E. glandulosa metabolism at the protein level. Conversely, in the metabolome, differential effects of the orientation of replacement interactions were only found following along-vessel interactions. No differences in expression were found in any compounds following across-vessel wood capture from either species. Following along-vessel capture however, concentrations of 27 compounds were found to be differentially expressed including increased levels of D-mannitol and D-xylitol in wood captured from $S$. rugosum. Following along-vessel replacements in general, replacement interactions with S. rugusom resulted in largely increased metabolite concentrations compared with those involving O. mucida. In particular the concentrations of D-mannitol and D-xylitol were increased in S. rugosum along-vessel replacement treatments. The increase in concentration of D-xylitol may suggest increased access to and degradation of hemicellulose by E. glandulosa under this treatment. Xylitol is the sugar alcohol derivative of the reduction of hemicellulose to the monosaccharide xylose (Converti et al. 1999). Whether this was due to greater access to hemicellulose being facilitated by S. rugosum or because the specialised metabolism of the earlier-arriving species exerted an increased need to degrade hemicellulose polymers deserves future attention. However the increased concentration of D-mannitol, a common carbonstorage compound produced by microbial species (Wiemken 2007; Deveau et al. 2008; Ghoreishi \& Shahrestani 2009; Song \& Vieille 2009), along with D-xylitol suggests that E. glandulosa invested more highly in carbon acquisition and storage following replacement of this particular species in along-vessel interactions. An interactive effect of initial coloniser identity and wood structural complexity was found to increase the concentration of cellobiose in wood recaptured from S. rugosum in across-vessel interactions, providing further evidence that under this scenario E. glandulosa may have invested more heavily in carbon acquisition and displayed increased access to cellulose.

Environmental "harshness" can positively affect the strength of priority effects if: early- and late-arriving species display a high degree of similarity in the use of resources; if early-arriving species strongly affect the environment; or if the growth rate of late-arriving species is particularly affected by the environment (Vanette \& Fukami 2014). Across-vessel territory recapture interactions may thereby weaken priority effects through a "harshened" environment effected by increased structural complexity (O'Leary 2018). In the present study, greater wood structural complexity appeared to weaken priority effects at the metabolite level but not at the protein level. This is an interesting metabolic response by E. glandulosa to the need to replace earlier-arriving species in order to secure nutrients and space and warrants further investigation. While the present study has clearly illustrated the utility of 'omics technologies in 
examining molecular mechanisms of priority effects, this differential response at protein and metabolite levels also illustrates that examining organismal biochemistry at multiple levels is important when considering the impact that priority effects have on metabolism.

There was evidence in the proteome of E. glandulosa that the vast majority of the species' enzymes were expressed through specialised metabolism and the relative proportion of enzymes expressed through this pathway did not change as a result of historical biotic interactions. Thus at the point of harvest $E$. glandulosa was likely investing principally in enzymes related to the decomposition of the lignocellulose resource and any toxic by-products of the degradation of both lignocellulose and any secondary metabolites produced by either species prior to and during replacement interactions. There was evidence in the metabolome of several organic acids, particularly succinic acid but also citric acid, succinic acid, sebacic acid and malic acid, in driving differences between the metabolome of E. glandulosa following secondary resource capture and following primary resource capture. Increased concentrations of organic acids, including malic acid, have been linked to a defensive response of Stereum hirsutum and competitors (Peiris et al. 2008) and the ability of ligninolytic fungi as with other microbes confers a competitive advantage particularly against species that cannot tolerate low $\mathrm{pH}$ (Magnuson \& Lasure 2004). A low $\mathrm{pH}$ environment is also important however for the activation of chelators used in the degradation of lignocellulose and phenolic compounds within wood (Goodell et al. 1997). Future work is needed to examine the dual role organic acids play in mediating biotic and abiotic fungal interactions in wood.

In summary the results presented here provide the first evidence that both replacement interactions and the structural complexity of the resource have a lasting impact on the metabolism of later-arriving species. These longer-term effects may lead to a change in growth and fitness in the later-arriving species which, in turn, may affect the outcomes of future biotic and abiotic interactions and community function. There remains considerable potential to expand this work and future studies should focus on (1) whether these changes in metabolism in the later-arriving species are congruent with a differential rate of replacement of the original coloniser and (2) to what extent do the changes affect the establishment success of a tertiary invader e.g. a species associated with the secondary succession such as T. versicolor or S. rugosum. The low rate of identification of low molecular weight metabolites here indicate that future work may benefit from the coupling of GC-MS with another system such as HPLCMS or NMR. 


\section{Chapter 6 - Metabolic responses of two pioneer ligninolytic fungi to variable temperature during fungal-wood interactions}

\subsection{Introduction}

During the decomposition of woody resources, all wood degrading fungi are exposed to fluctuations in environmental conditions, especially temperature, water availability, gaseous regime and $\mathrm{pH}$ (Magan 2008). In wood decay systems there is considerable niche overlap as many species compete for space within the same spatially defined resource (Boddy 2000). Fluctuating conditions have long been thought to promote coexistence in such situations (Hutchinson 1961) and environmental fluctuations have been linked experimentally to reduced extinction rates (Naeem \& Li 1997; Petchey et al. 1999). The pioneer community, being restricted to attached dead branches which are not buffered by the woodland floor, is particularly prone to fluctuating conditions (Unterseher \& Tal 2006). As such, pioneer species may be highly adapted to greater fluctuations in abiotic conditions.

Abiotic conditions may influence fungal growth, the production and activity of enzymes, interaction outcomes and ultimately, the identity of fungi present in a given community at a given time (Cartwright \& Findlay 1958; Magan 2008; Hiscox et al. 2016a). The growth rates of ligninolytic fungi across artificial media are known to be affected by ambient temperature (Boddy 1983). Manipulation of ambient temperature has been shown to alter the outcome of competitive interactions between fungi, with certain pioneer fungi exhibiting increased defensive ability against secondary successional species with increasing temperature (Hiscox et al. 2016a). Intricate changes in the proteomic profile of ligninolytic fungi have been demonstrated at increased temperature including changes in the enzymes utilised in detoxification of the environment, in combating oxidative stress and in decomposing lignocellulose and acquiring carbon. Of particular interest to ecosystem function, the cellulose- and hemicellulosemetabolising CAZyme profile produced by wood decay fungi is more diverse under increased temperature (Moody et al. 2018a). Thus, temperature clearly plays a role in the rate of decomposition of woody resources.

Fluctuating temperature in species-rich wood decay communities results in increased decomposition rate and supports greater species richness although in more species-rich systems, fluctuating temperature results in reduced metabolic efficiency by promoting increased fungal biomass production (Toljander et al. 2006). Thus, fluctuating temperature clearly contributes to driving important woodland ecosystem functions. Such temperature regimes may be responsible for the species richness observed in natural attached branch communities (Chapter 3; Unterseher \& Tal 2006) and the pioneers that associate 
with them may be capable of making a greater contribution to overall decomposition process under fluctuating environment. These dynamics have generally been overlooked however.

The present study investigated whether two different pioneer fungi with different ecological strategies responded differently at the protein and metabolite level to a diurnally fluctuating temperature regime compared with a stable one. Exidia glandulosa is known to be associated with attached wood undergoing severe abiotic stress, particularly high desiccated conditions (Boddy \& Rayner 1983b; Boddy 2001; Heilmann-Clausen 2001) and Oudemansiella mucida, a highly defensive species, was the most commonly isolated species from naturally occurring attached beech branches (Chapter 3). Untargeted metabolomics and proteomics were employed to place an emphasis on broadening our view of the proteins, metabolites and biochemical pathways employed by the fungi in their interactions with wood and their abiotic environment.

\subsection{Outline of methods}

\subsubsection{Experimental design and microcosm conditions}

Two fungal strains isolated from attached branch communities (Chapter 3) were selected based on their ecological roles: E. glandulosa due to stress-selection and O. mucida due to its prevalence in naturally formed communities previously surveyed (Chapter 3). Sterile beech wood blocks measuring $2 \mathrm{~cm}^{3}$ were pre-colonised by placing on $0.5 \%(\mathrm{w} / \mathrm{v})$ MEA cultures of each fungal strain and incubating in the dark for 8 weeks. Mycelia and agar were then scraped from the surface of each block and blocks were combined in single-species $2 \times 2$ block configurations (Section 2.5.2). Microcosms were each provided 10 $\mu \mathrm{l}$ autoclaved distilled $\mathrm{H}_{2} \mathrm{O}$ at experimental set-up but to ensure the findings from the study were comparable with those of Chapter 5, microcosms were not watered after this point. Microcosms were incubated at either $15^{\circ} \mathrm{C}$ or under a diurnal temperature regime where they were held at $5^{\circ} \mathrm{C}, 15^{\circ} \mathrm{C}, 25^{\circ} \mathrm{C}$ then $15^{\circ} \mathrm{C}$ for 6 hours per temperature every 24 hours. Each treatment (species; temperature) was replicated three times $(n=12)$. The experiment was run for 8 weeks after which wood blocks were broken up into thirds for re-isolations (Section 2.5.3), GC-MS metabolome analysis (Section 2.6) and LCMS/MS proteome analysis (Section 2.7).

\subsubsection{Data analyses}

\subsubsection{Untargeted GC-MS metabolomics}

GC-MS chromatogram features were pre-processed, deconvoluted, aligned and assigned putative identifications using AMDIS (D'Arcy \& Mallard 2004), SpectConnect (Flory et al. 2007) and the NIST 11 (Wiley) and GMD (Kopka et al. 2005) spectral databases (Section 2.6.3). Metabolites conserved across two out of three replicates in each tretmant group were reported using SpectConnect (Flory et al. 2007). 
All data analyses were undertaken in MetaboAnalyst 4.0 (Chong et al. 2018). PLS-DA models were built for O. mucida and E. glandulosa to visualise and assess the degree to which metabolite identities and concentrations adequately separate samples into their treatment groups. Important compounds contributing to the separation of samples into their respective treatment groups were identified by VIP analysis, wherein compounds with VIP scores $>1.0$ were considered to be important discriminators (section 2.6.4.2).

T-tests with a 5\% Benjamini-Hochberg FDR correction for multiple comparisons (Benjamini \& Hochberg 1995) were applied to test the null hypothesis of no significant differences in compound concentration between experimental conditions. Results of T-tests were combined with fold-change analysis using volcano plots (fold change threshold 2.0; raw P-value threshold 0.05) to visualise whether metabolites tended to be expressed with increased or decreased concentrations under variable compared with stable temperature (Section 2.6.4.2).

\subsubsection{Untargeted LC-MS/MS proteomics}

LC-MS/MS detected proteins were analysed using the predicted protein databases of E. glandulosa HHB12029 (Nagy et al. 2016) and O. mucida CBS55879 (Barrasa 2014) using MaxQuant (version 1.6.2.3) with integrated Andromeda for database searching (Cox \& Mann 2008; Section 2.7.2). Functional annotation and the mapping of annotated enzymes to KEGG pathways was undertaken in B2G (Section 2.7.3).

Putatively identified proteins were imported into MetaboAnalyst 4.0 (Chong et al. 2018) for differential analysis. To visualise and assess the degree to which protein identities and concentrations adequately separate samples into their treatment groups PLS-DA models were built for the O. mucida and E. glandulosa experiments. Important compounds contributing to the separation of samples into their respective treatment groups were identified by VIP analysis, wherein compounds with VIP scores $>1.0$ were considered to be important discriminators (section 2.7.4.2).

T-tests with a 5\% Benjamini-Hochberg false discovery rate (FDR) correction for multiple comparisons (Benjamini \& Hochberg 1995) were applied to test the null hypothesis of no significant differences in compound concentration between experimental conditions. Results of T-tests were combined with foldchange analysis using volcano plots (fold change threshold 2.0; raw P-value threshold 0.05) to visualise whether metabolites tended to be expressed with increased or decreased concentrations under variable compared with stable temperature (Section 2.7.4.2). 


\subsection{Results}

\subsubsection{GC-MS metabolomics of Oudemansiella mucida and Exidia glandulosa under stable and variable temperature}

SpectConnect identified a total of 540 conserved compounds across the two experiments $(O$. mucida and E. glandulosa). Of these, 55 compounds were putatively identified for the two organisms and matched to metabolites (Appendix III) including 18 fatty acids, 9 saccharides (carbohydrates), 7 organic acids, 7 sugar alcohols, 5 phenols, 3 aromatic acids, 3 sugar acids, 2 aromatic alcohols, 1 mineral acid and one each of aldehydes and terpenoids. Three identified metabolites were uniquely associated with $O$. mucida, 12 uniquely with $E$. glandulosa and 39 were produced by both species. Compound classification identified 21 associated with carbohydrate metabolism, 8 with energy metabolism, 8 with lipid metabolism, 4 with polyphenol metabolism, 4 with xenobiotics biodegradation and metabolism, 3 with amino acid metabolism, 3 with biosynthesis of secondary metabolites, and one each with degradation of aromatic compounds and metabolism of cofactors and vitamins. A single compound, thiodilactic acid, could not be assigned to a metabolite class or biological function.

\subsubsection{Effect of variable temperature on the metabolomes of Oudemansiella mucida and Exidia glandulosa}

Samples could be separated into variable and stable temperature treatments based on the variation in the metabolome of both O. mucida and E. glandulosa, however models were over-fitted in both cases (PLS-DA; Fig. 6.1 \& 6.3). In O. mucida the primary axis separated samples based on temperature treatment and explained $27.0 \%$ of the metabolic variation. Component 2 explained a further $27.8 \%$ of observed variation (Full model $=3$ components; R2 = 1.0; $Q 2=-0.02 ;$ Accuracy: 0.50; Fig. 6.3). The identity and concentration of 110 compounds contributed to the separation of samples colonised by $O$. mucida, including the putatively identified metabolites 2-oxobutanoic acid and glyceraldehyde (VIP score > 1.0; Appendix III). The greatest degree of variation in the metabolome of $O$. mucida could be explained by variation in the concentration of unidentified compound TO486 and this, along with the next four most important compounds all had higher relative concentration under the variable regime. A further 109 compounds including the putatively identified metabolites 2-oxobutanoic acid and glyceraldehyde also contributed to sample separation into respective treatment groups. The O. mucida metabolome was subject to considerably less variation between samples than under the variable treatment. 


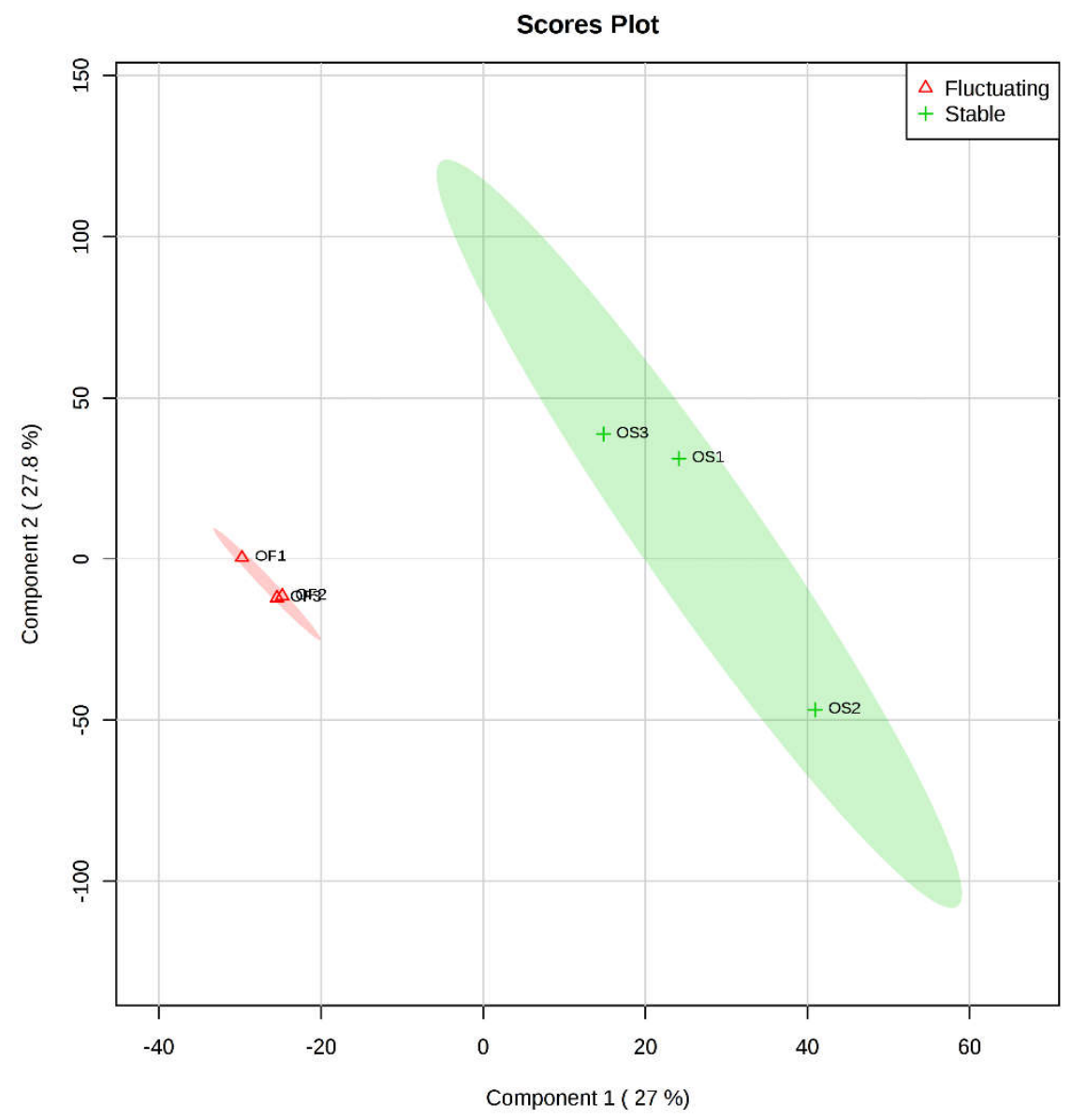

Figure 6.1 PLS-DA of the metabolome of Oudemansiella mucida under stable and variable temperature regimes. Red, diurnally fluctuating temperature $\operatorname{ramp}\left(5^{\circ} \mathrm{C}, 15^{\circ} \mathrm{C}, 25^{\circ} \mathrm{C}\right.$ in 6 hour blocks every 24 hours); green, stable temperature regime $\left(15^{\circ} \mathrm{C}\right)$; shaded areas, $95 \%$ confidence limits.

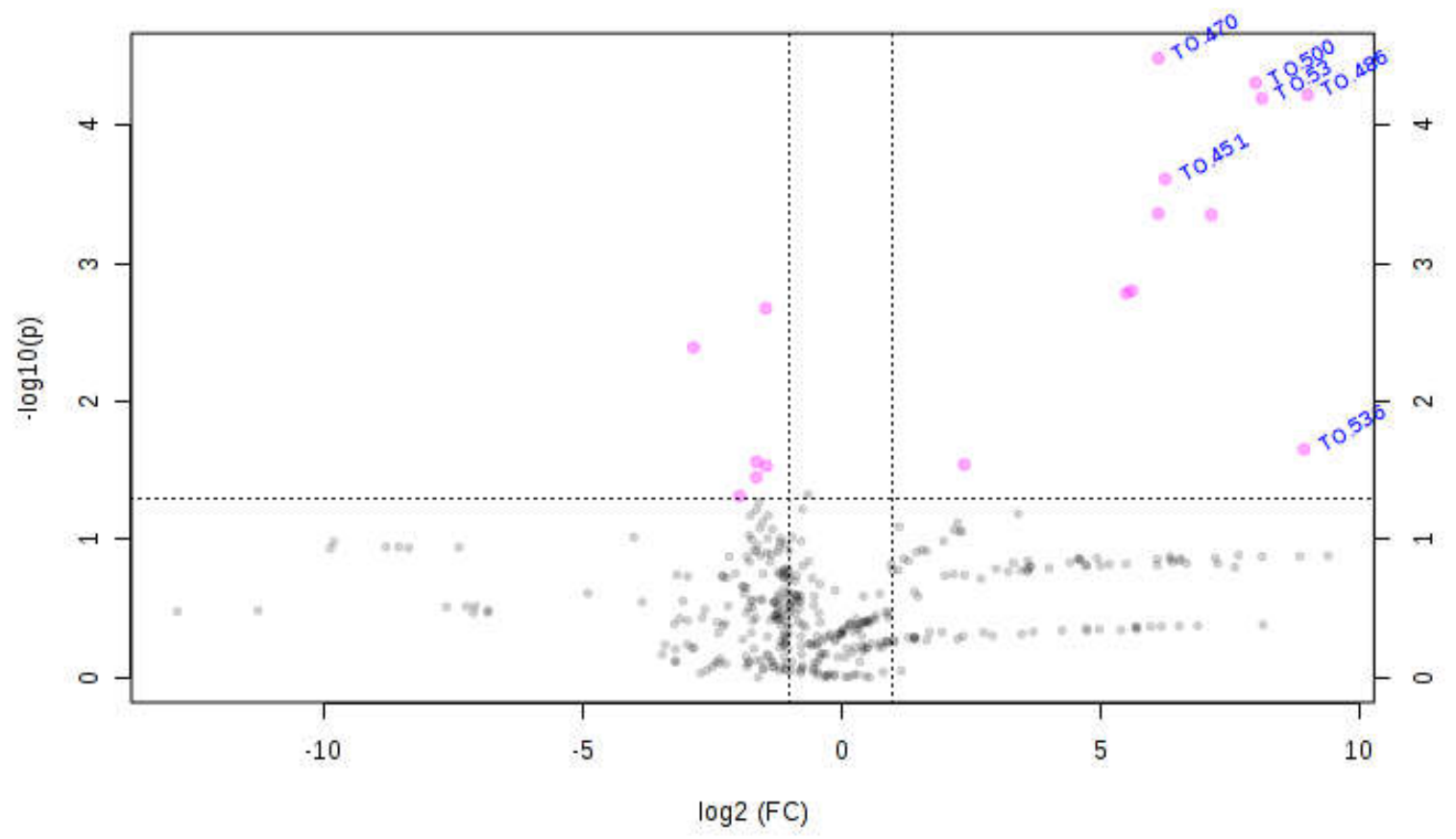

Figure 6.2 Volcano plot of Oudemansiella mucida metabolome under variable climate temperature compared with stable temperature

Pink, compounds exhibiting <-2.0 (left) or $>2.0$ (right) fold change under the variable temperature regime; blue labels, compounds with significantly different concentrations in the variable temperature treatment (T-test; P $<0.05$ with $5 \%$ Benjamini-Hochberg FDR correction for multiple comparisons). 
Table 6.1 T-tests of compound concentrations in the metabolome of Oudemansiella mucida under variable temperature compared with stable temperature

P value, raw P-value; FDR, 5\% Benjamini-Hochberg FDR corrected P-values due to multiple comparisons (Benjamini \& Hochberg 1995)

\begin{tabular}{lcccc}
\hline Compound & T statistic & P value & $\mathbf{- 1 0 L o g ( P - v a l u e )}$ & FDR corrected P \\
\hline TO.470 & 20.618 & $3.27 \mathrm{E}-05$ & 4.4856 & 0.006197 \\
TO.500 & 18.591 & $4.93 \mathrm{E}-05$ & 4.3074 & 0.006197 \\
TO.486 & 17.677 & $6.02 \mathrm{E}-05$ & 4.2207 & 0.006197 \\
TO.53 & 17.41 & $6.39 \mathrm{E}-05$ & 4.1946 & 0.006197 \\
TO.451 & 12.379 & 0.000245 & 3.6113 & 0.018991 \\
TO.510 & 10.673 & 0.000437 & 3.36 & 0.024689 \\
TO.518 & 10.618 & 0.000445 & 3.3512 & 0.024689 \\
\hline
\end{tabular}

The concentrations of 7 unidentified compounds in the metabolome of O. mucida (compounds TO470, TO500, TO486, TO53, TO451, TO518 and TO510) were highly significantly different between the stable and variable temperature groups and all seven had higher relative concentration under the variable temperature regime (T-tests; $\mathrm{P}<0.001$; Table 6.1 and Fig. 6.2).

The metabolome of in samples colonised by E. glandulosa were separated into treatment groups by a PLS-DA model comprising 3 components which together explained $67.1 \%$ variance. The primary axis separated samples by temperature treatment and explained $23.7 \%$ of the observed metabolic variation. Component 2 explained a further 19.4\% of variation $(\mathrm{R} 2=1.0 ; \mathrm{Q} 2=-0.05$; Accuracy = 0.50; Appendix III). Unidentified compound TE311 was responsible for the greatest degree of variation in the E. glandulosa metabolome and had lower relative concentration in the variable temperature group (Appendix III). A further 113 compounds including the putatively identified metabolites succinic acid, boric acid, protocatechuic acid, 1,2,4-butanetriol and erythronic acid also contributed to sample separation in PLSDA (VIP score > 1.0; Appendix III).

Table 6.2 T-tests of compound concentrations in the metabolome of Exidia glandulosa under variable temperature compared with stable temperature

P value, raw P-value; FDR, 5\% Benjamini-Hochberg FDR corrected P-values due to multiple comparisons (Benjamini \& Hochberg 1995)

\begin{tabular}{lrrrr}
\hline Compound & T statistic & \multicolumn{1}{c}{ P value } & -LOG10(p) & FDR corrected P \\
\hline TE.300 & -18.642 & $4.87 \mathrm{E}-05$ & 4.3121 & 0.008542 \\
TE.302 & -17.504 & $6.25 \mathrm{E}-05$ & 4.2038 & 0.008542 \\
TE.324 & -17.136 & $6.80 \mathrm{E}-05$ & 4.1673 & 0.008542 \\
TE.311 & -15.854 & $9.25 \mathrm{E}-05$ & 4.0338 & 0.008542 \\
TE.314 & -14.903 & 0.000118 & 3.9279 & 0.008542 \\
TE.70 & 14.456 & 0.000133 & 3.8758 & 0.008542 \\
TE.77 & 12.362 & 0.000246 & 3.609 & 0.012164 \\
TE.28 & 12.278 & 0.000253 & 3.5973 & 0.012164 \\
TE.128 & 11.268 & 0.000353 & 3.4518 & 0.014155 \\
TE.69 & 11.154 & 0.000368 & 3.4346 & 0.014155 \\
\hline
\end{tabular}




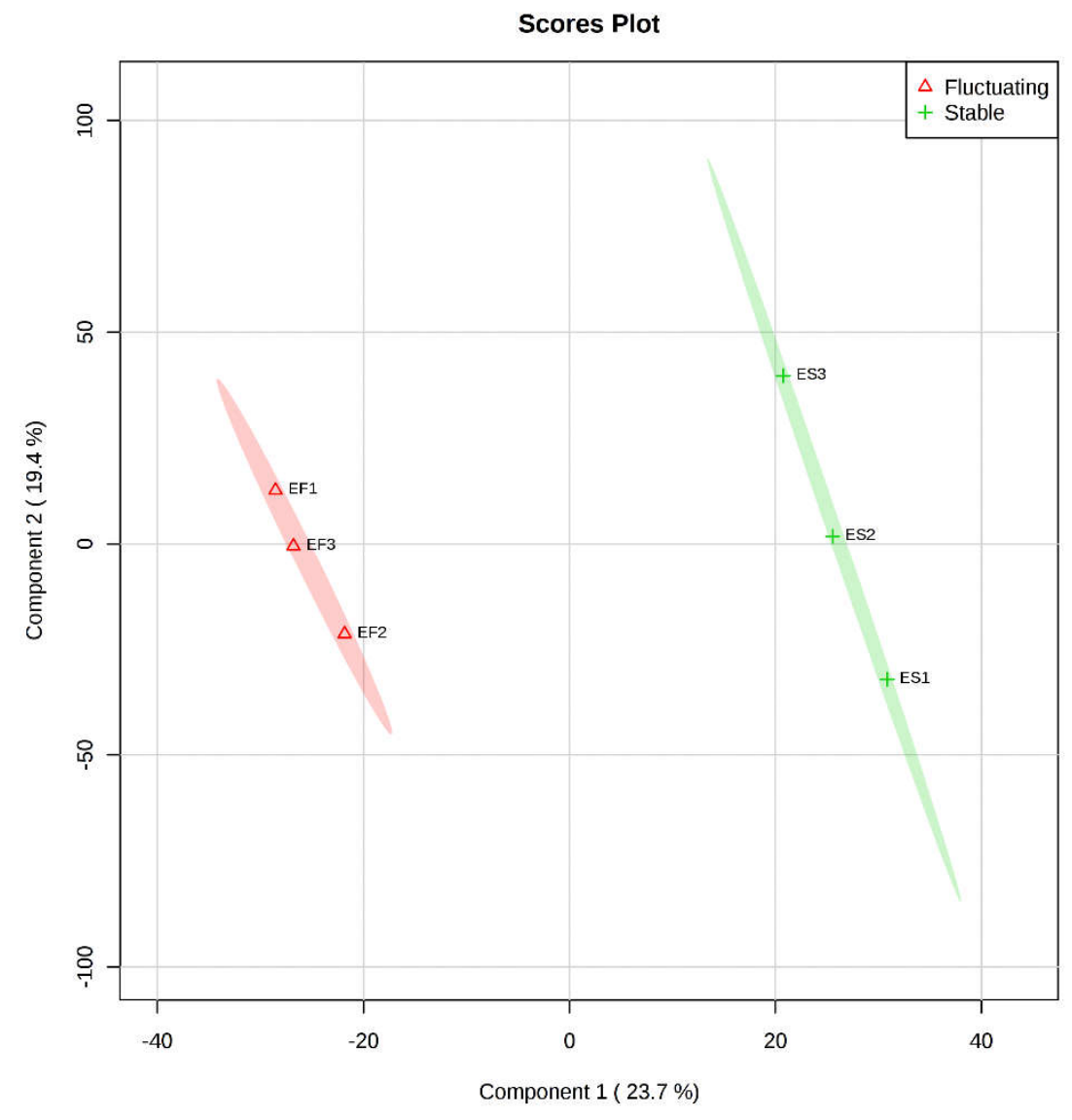

Figure 6.3 PLS-DA of the metabolome of Exidia glandulosa under stable and variable temperature regimes.

Red, diurnally fluctuating temperature ramp $\left(5^{\circ} \mathrm{C}, 15^{\circ} \mathrm{C}, 25^{\circ} \mathrm{C}\right.$ in 6 hour blocks every 24 hours); green, stable temperature regime $\left(15^{\circ} \mathrm{C}\right)$; shaded areas, $95 \%$ confidence limits.

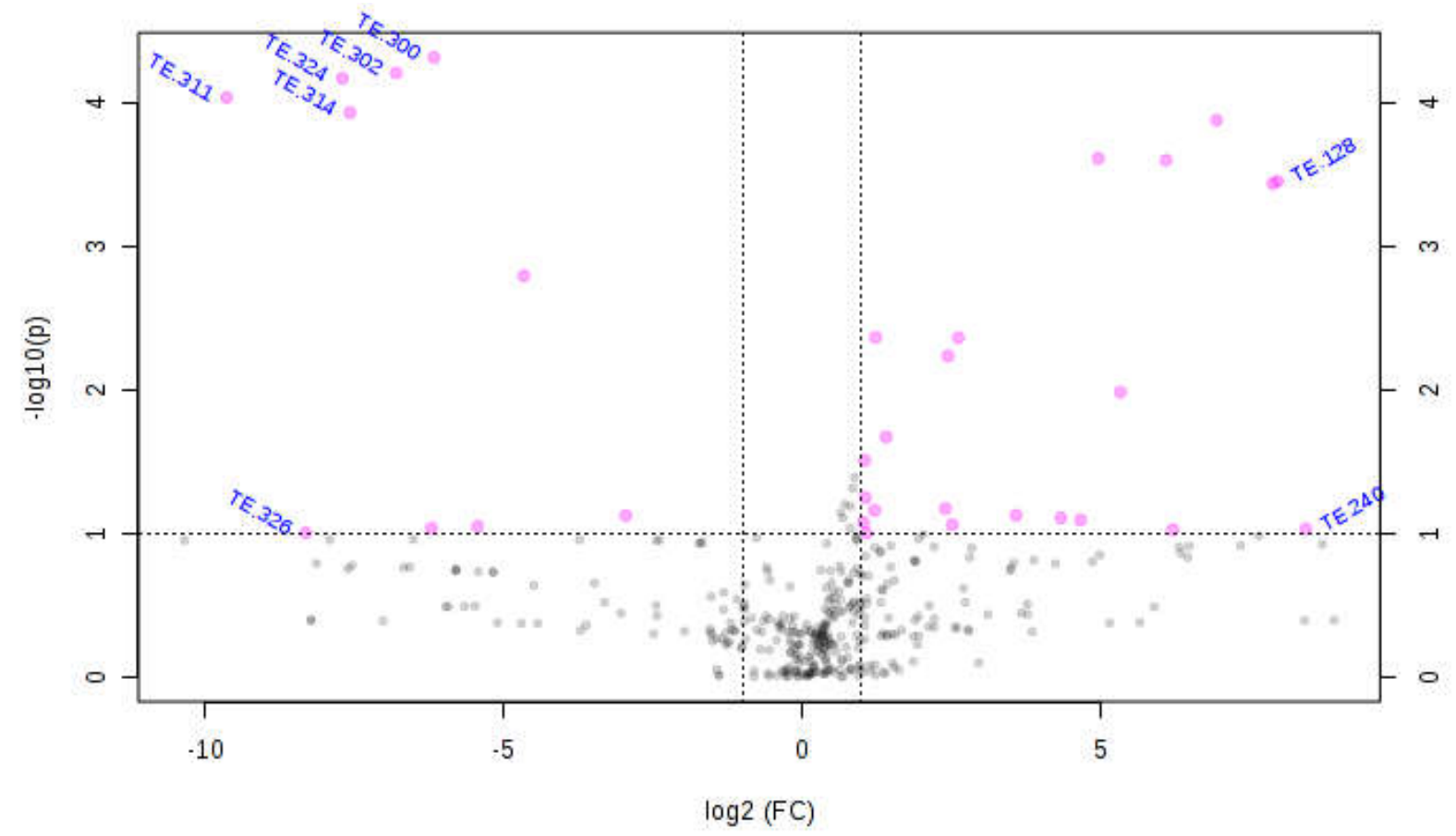

Figure 6.4 Volcano plot of Exidia glandulosa metabolome under variable climate temperature compared with stable temperature

Pink, compounds exhibiting < -2.0 (left) or > 2.0 (right) fold change under the variable temperature regime; blue labels, compounds with significantly different concentrations in the variable temperature treatment (T-test; $\mathrm{P}<0.05$ with $5 \%$ Benjamini-Hochberg FDR correction for multiple comparisons). 
The concentrations of 10 unidentified compounds (compounds TE300, TE302, TE324, TE311, TE314, TE70, TE77, TE28, TE128 and TE69) were highly significantly different between the temperature regime treatments. Of these compounds TE128 and TE240 had higher relative concentration under the variable temperature regime with the others exhibiting higher concentration under stable temperature (T-tests; P $<0.001$; Table 5.2 and Fig 6.4).

\subsubsection{Functional annotation of Oudemansiella mucida and Exidia glandulosa proteomes analysed by direct shotgun LC-MS/MS}

MaxQuant identified the sequences of 587 proteins associated with all samples; 542 proteins were associated with samples colonised by O. mucida and 108 proteins were associated with samples colonised by E. glandulosa (Appendix IV). Of 587 proteins, 542 successfully completed the B2G GO pipeline including GO slimming.

Exploration of functional classifications indicated that there was little difference in strategy in either species' response to variable and stable temperature regime (Appendix IV). However there appeared to be some differences in strategy between the two species under the same temperature treatment. For example, under the variable temperature regime when classified by biological process (ontology level 5), E. glandulosa produced a more varied proteome than O. mucida. While there was little difference in the major processes (cellular protein metabolism, gene expression, macromolecule biosynthesis, organonitrogen compound biosynthesis, peptide metabolism, cellular amide metabolism, and cellular macromolecule biosynthesis all contributing approximately $10 \%$ to the overall proteome), E. glandulosa uniquely expressed proteins associated with catabolism of nucleobase-containing compounds, heterocycle catabolism, catabolism of organic cycle compounds, catabolism of aromatic compounds, protein modification and macromolecule modification. O. mucida uniquely expressed proteins associated with oxoacid metabolism and a greater proportion of proteins associated with biosynthesis of cellular nitrogen compared with E. glandulosa. When classifying the proteome by molecular function, $E$. glandulosa uniquely expressed proteins associated with organic cyclic compound binding and heterocyclic compound binding.

EC codes were assigned which identified sequences mapping to 195 enzymes associated with O. mucida and 31 enzymes associated with E. glandulosa (Fig. 6.8 and Appendix IV). The majority of enzymes were hydrolases (O. mucida: $n=88$; E. glandulosa: $n=16)$. The distribution pattern of enzymes differed between the two species in that the $O$. mucida proteome expressed considerable variation in the distribution of oxidoreductases $(n=45)$, transferases $(n=25)$ and hydrolases $(n=88)$ whereas $E$. glandulosa expressed the same three groups of enzymes with roughly equal abundance $(n=13 ; n=14$; and $n=16$ respectively). 
Of the 195 enzymes associated with O. mucida 45 mapped to the KEGG pathway corresponding with biosynthesis and metabolism of antibiotics under the stable temperature condition and 44 with the same pathway under the variable temperature condition (Appendix IV). In addition, three enzymes mapped to the pathway corresponding with cyanoamino acid metabolism under both temperature treatments. There was a single considerable difference between the two temperature treatments in the O. mucida proteomes in that 10 enzymes mapped to the pathway corresponding with pyruvate metabolism under the stable temperature condition but no enzymes associated with this pathway were present in samples associated with the variable condition. In E. glandulosa proteomes 18 enzymes mapped to the pathway corresponding with biosynthesis of antibiotics under both temperature conditions and enzymes associated with glycolysis, purine metabolism, the pentose-phosphate shunt and the TCA cycle were also well represented.

\subsubsection{Differential expression analysis of Exidia glandulosa and Oudemansiella mucida proteomes under variable and stable temperature}

Samples could be separated into variable and stable temperature treatments based on the identity and abundance of proteins for O. mucida but not for E. glandulosa. PLS-DA models were based on 542 proteins in the case of $O$. mucida and, in a model with three components $(\mathrm{R} 2=1.00 ; \mathrm{Q} 2=0.71$; Accuracy = 1.00; Appendix IV), the first component separated samples into treatment groups and explained $42.5 \%$ of the variation in the proteome with component 2 explaining a further $32.1 \%$ of variation (Fig. 6.2). The abundance of 115 proteins contributed to the separation of samples into treatment groups in the model (Appendix IV). Of these, an erylysin B (jgiOudmuc11429374gm1.2232_g) contributed most to sample separation followed by a carbonic anhydrase (jgiOudmuc11372311fgenesh1_pm.2_\#_67), a GMC oxidoreductase (jgiOudmuc 11230519e_gw1.160.67.1), an unknown protein (jgiOudmuc11278158fgenesh1_kg.7_\#_455_\#_TRINITY_DN6792_c0_g1_i4), a 40S ribosomal protein S15 (jgiOudmuc1499056CE499055_39586) and a translationally controlled tumour associated protein (jgiOudmuc11218923e_gw1.30.298.1) all of which had higher relative concentration under the stable temperature regime. A calmodulin (jgiExigl1766531gm1.8512_g), a 40S ribosomal protein S17 (jgiOudmuc11324722fgenesh1_kg.119_\#_75_\#_TRINITY_DN3064_c0_g2_i3) and a 20S proteasome subunit protein (jgiExigl1741341 estExt_fgenesh1_pm.C_2380005) also contributed substantially to sample separation and had higher relative concentration under the variable temperature regime. In the case of E. glandulosa, PLS-DA models (Fig 6.6 and Appendix IV) were overfitted as indicated by negative Q2 values, a situation which could not be improved by either adding or removing components from the model. 


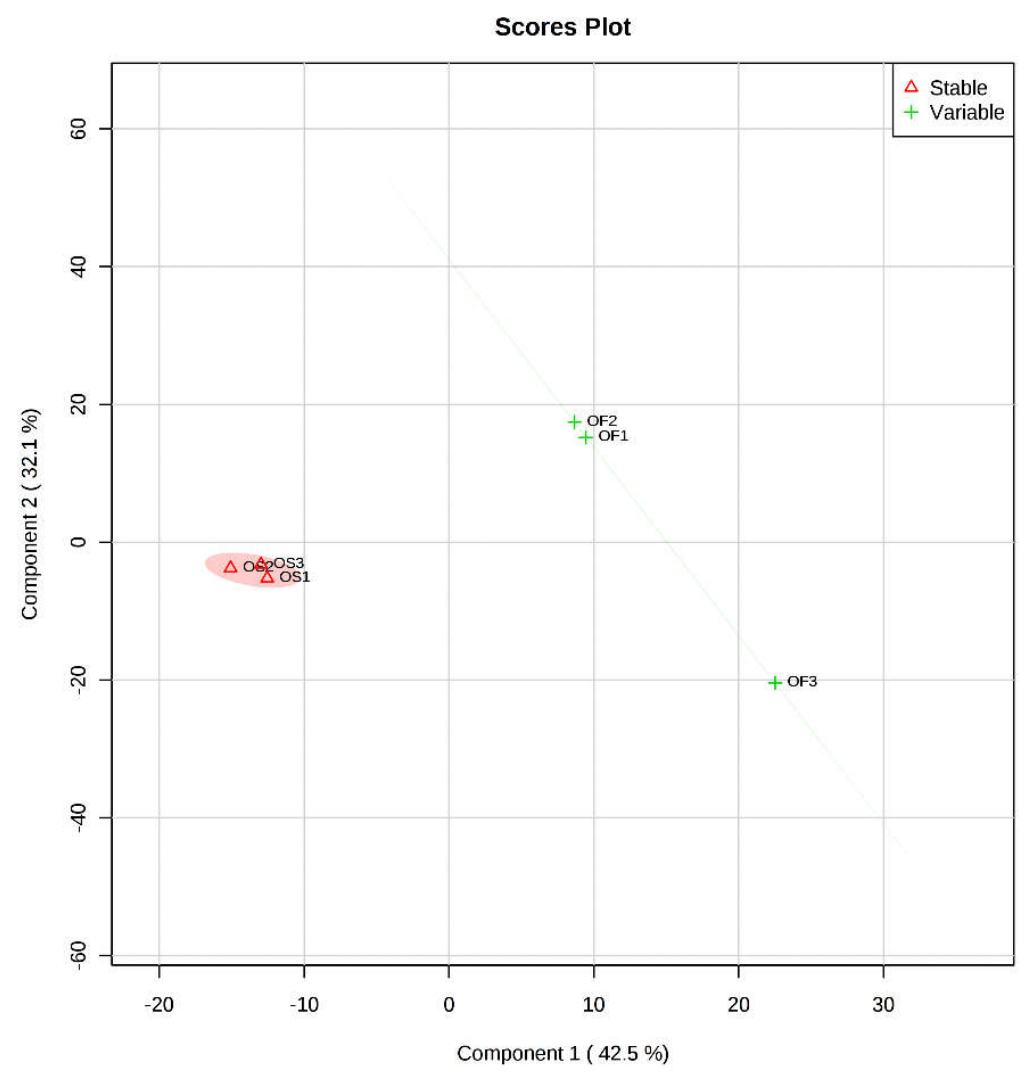

Figure 6.5 PLS-DA of Oudemansiella mucida proteome under stable and variable temperature regimens Red, diurnally fluctuating temperature $\operatorname{ramp}\left(5^{\circ} \mathrm{C}, 15^{\circ} \mathrm{C}, 2^{\circ} \mathrm{C}\right.$ in 6 hour blocks every 24 hours); green, stable temperature regime $\left(15^{\circ} \mathrm{C}\right)$; shaded areas, $95 \%$ confidence limits.

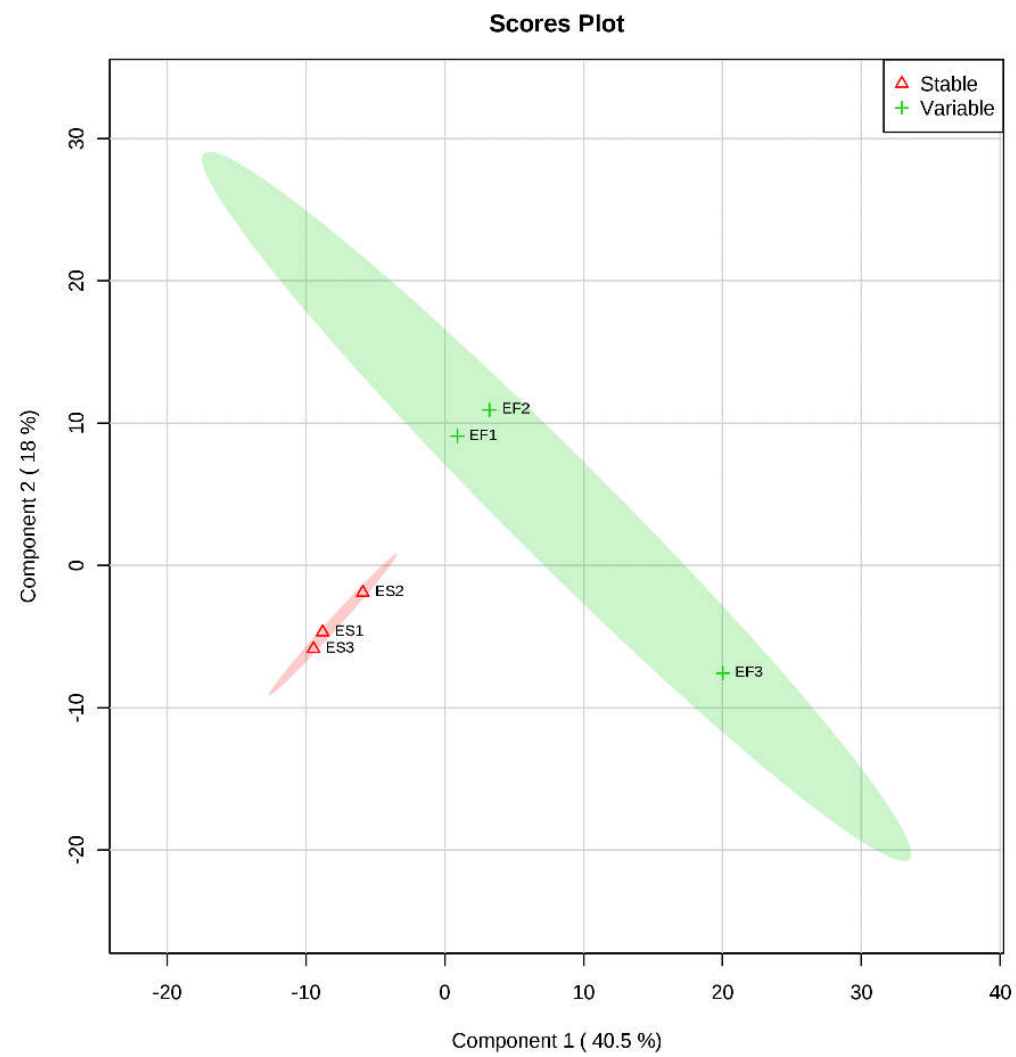

Figure 6.6 PLS-DA of Exidia glandulosa proteome under stable and variable temperature regimens

Red, diurnally fluctuating temperature ramp $\left(5^{\circ} \mathrm{C}, 15^{\circ} \mathrm{C}, 25^{\circ} \mathrm{C}\right.$ in 6 hour blocks every 24 hours); green, stable temperature regime $\left(15^{\circ} \mathrm{C}\right)$; shaded areas, $95 \%$ confidence limits. 
O. mucida produced differential expression of 19 proteins between the stable and variable temperature treatment groups (Fig 6.7, Appendix IV). Of these a 20 S proteasome subunit protein (jgiExigl1741341estExt_fgenesh1_pm.C_ 2380005), an oligoxyloglucan reducing end-specific cellobiohydrolase (jgiOudmuc11267226fgenesh1_kg.1_\#_2754_\#_TRINITY_DN6639_c0_g1_i5) and a 40S ribosomal protein S17 (jgiOudmuc11324722 fgenesh1_kg.119_\#_75_\#_TRINITY_DN3064_c0_g2_i3) had lower relative concentration under the variable temperature with the remaining 16 differentially expressed proteins having higher relative concentration. The three proteins exhibiting the greatest fold changes were an erylysin B protein ( $\mathrm{FC}=237.6$; $\mathrm{P}<0.001$; jgiOudmuc11372311fgenesh1_pm.2_\#_67), a GMC oxidoreductase $(\mathrm{FC}=79.5 ; \mathrm{P}<0.001$; jgiOudmuc11230519e_gw1.160.67.1) and an unidentified protein (FC = 47.2; P < 0.001; jgiOudmuc11278158fgenesh1_kg.7_\#_455_\#_TRINITY_DN6792_c0_g1_i4), all of which had been identified by PLS-DA VIP as being important in sample separation.

E. glandulosa protein expression did not differ between the temperature treatment groups as indicated by over-fitted PLS-DA models and no significant differences in the concentrations of any of the detected proteins as assessed by T-test analysis.

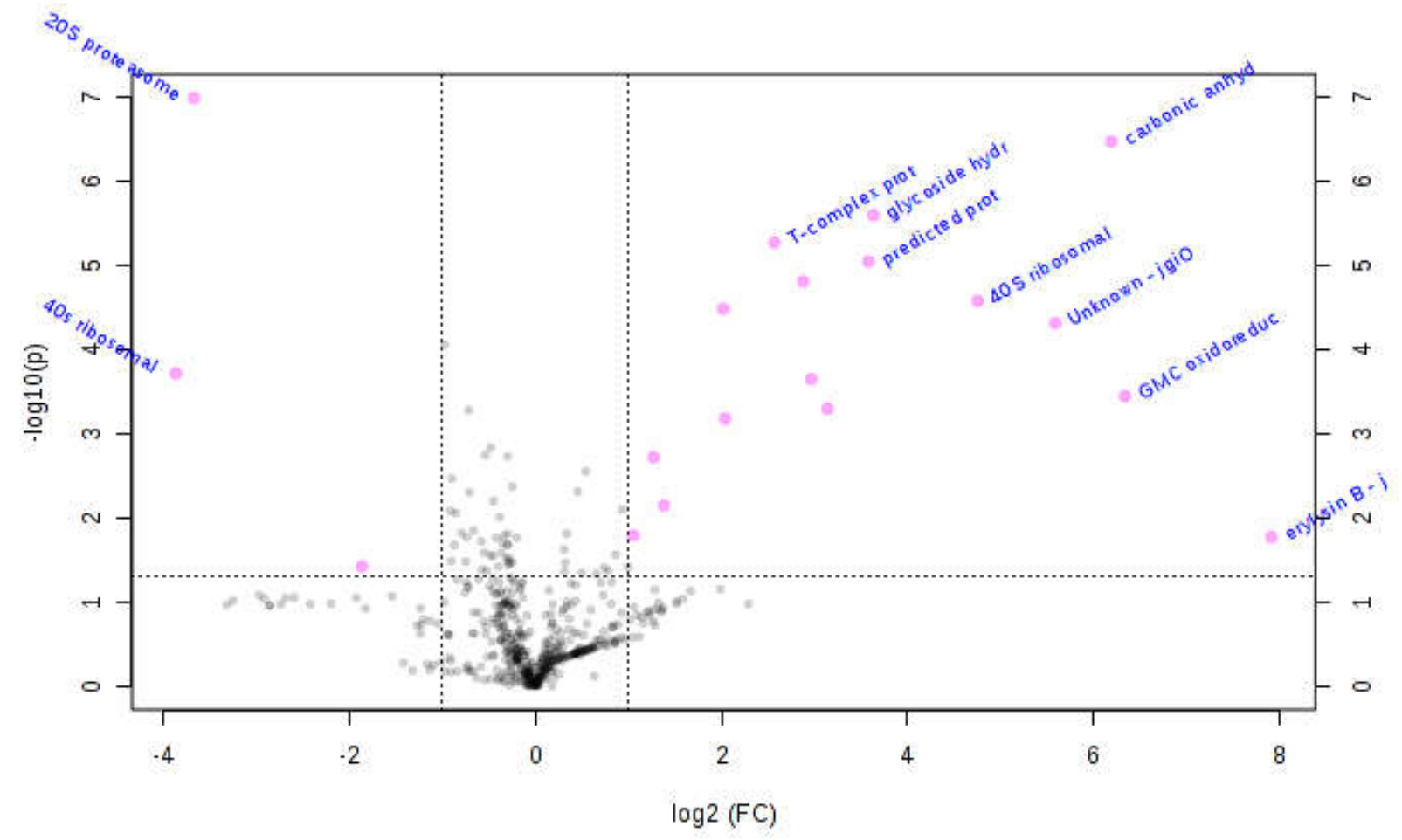

Figure 6.7 Volcano plot of Oudemansiella mucida proteome under variable climate temperature compared with stable temperature

Pink, compounds exhibiting < - 2.0 (left) or $>2.0$ (right) fold change under the variable temperature regime; blue labels, compounds with significantly different concentrations in the variable temperature treatment (T-test; $\mathrm{P}<0.05$ with $5 \%$ Benjamini-Hochberg FDR correction for multiple comparisons). 


\subsection{Discussion}

The proteome and metabolome of $O$. mucida were affected by variable temperatures, with most proteins and metabolites being present in greater abundance under the variable regime. Of the 16 proteins with greater relative concentration under the variable temperature regime, 10 were enzymes. These included a GMC oxidoreductase which may be implicated in cellobiose degradation (Henriksson et al. 2000), an oligoxyloglucan reducing end-specific cellobiohydrolase which is involved in the hydrolysis of cellobiose from the hemicellulose xyloglucan (Yaoi \& Mitsuishi 2002) and a member of the CAZyme glycoside hydrolase family 31 which play a central role in carbohydrate metabolism indicating that variable temperature may facilitate enhanced acquisition of nutrients through lignocellulose decomposition in this species. Other enzymes with higher relative concentration play central roles in energy metabolism such as carbonic anhydratase (Elleuche \& Poggeler 2009; Elleuche \& Pöggeler 2010) and thiolase; or amino acid metabolism such as an aldo-keto reductase (Bohren et al. 1989) and a serine carboxypeptidase, likely involved in proteolysis (Breddam 1986). The compounds 2-oxobutanoic acid and glyceraldehyde were important in distinguishing between $O$. mucida colonised samples under the two temperature regimes. 2-oxobutanoic acid, also known as a-ketobutanoic acid, is produced during amino acid metabolism, specifically following lysis of cystathionine and as a degradation product of both threonine and methionine (Frolkis et al. 2009). Glyceraldehyde is a monosaccharide and a constituent of wood lignins including beech lignin (Nimz 1974). In O. mucida colonised wood, almost all compounds found to significantly differ between treatment groups were found in greater concentration under variable temperature.

O. mucida, which dominates pioneer communities in natural beech systems (Chapter 3) seemed to responded to variable temperature to a greater extent than the stress-selected pioneer E. glandulosa at both the protein and metabolite levels. In particular, cellobiohydrolase and erylysin were present in higher abundance under variable temperature temperature in the proteome of $O$. mucida indicating that investment in nutrient acquisition processes may have increased. Under stable temperature, a $20 \mathrm{~S}$ proteasome subunit protein and 40S ribosomal protein S17 protein were present in higher abundance indicating that growth processes of the fungus may have been reduced with an increase in proteasome and ribosomal protein turnover and degradation (Bhattacharyya et al. 2014; Mathieson et al. 2018) and these processes may also be linked to nutrient acquisition (Mathis et al. 2017). In contrast, the protein profile of E. glandulosa did not differ at all between the two regimes and but the concentrations of a number of metabolic compounds in its metabolome were significantly affected. The vast majority of which were expressed in lower concentrations under the variable regime potentially indicating that it struggled to cope with stable temperature to a greater degree. Amongst the compounds contributing to separation of samples colonised by E. glandulosa into temperature treatment groups, were the putatively identified metabolites succinic acid (succinate; butanedioic acid), protocatechuic acid, erythronic acid 
and 1,2,4-butanetriol. As such, these metabolites along with the unidentified compounds found to be expressed in significantly different concentrations under the two temperature regimes may contribute to E. glandulosa's ability to withstand stressful abiotic conditions in wood and may help inform future hypotheses and targeted studies. Succinate is central to energy metabolism in all organisms as an intermediate in both the TCA and glyoxylate cycles and also plays an important role in signal transduction and gene expression (Tretter et al. 2016). Protocatechuic acid is a phenol commonly found in plants (Nakamura et al. 2000) while 1,2,4-butanetriol (Xie et al. 2014) and erythronic acid (Shao et al. 2008) are likely to be break down products of lignin.

Taken together these results show that the metabolism of O. mucida is affected to a greater degree at both the protein and metabolite level by variable temperature than E. glandulosa. O. mucida did not express any enzymes associated with pyruvate metabolism in wood subjected to variable temperature indicating that variable temperatures could have a severe impact on carbon metabolism in some ligninolytic fungi (Pronk et al. 1996). There is clearly an impact of variable temperature on the metabolism of the fungi studied here, one of which is known to be a stress-selected species. The results suggest that E. glandulosa, of the two, may have been better able to cope with stable temperature given that the proteome of this species was not affected. Thus, in this stress-selected species, protein profiles in wood may be driven to a greater extent by nutrient acquisition processes than in the less stress-tolerant species. As such, stresstolerant species such as E. glandulosa may be capable of contributing more significantly to decomposition processes under stable conditions which may have implications for future experimental work as well as in biorefining applications.

PLS-DA models for the metabolome of both species and the proteome of E. glandulosa were slightly overfitted suggesting that variation in metabolism of both species at various levels was predominantly being driven by factors other than temperature regime. The desiccated conditions provided may have contributed significantly to the organisms' variation in metabolic response, however following biotic interactions (Chapter 5) PLS-DA models indicated that samples could legitimately be separated into treatment groups to explain a considerable proportion of variance. Taken together these markedly different responses may indicate that neither pioneer species is particularly sensitive to temperatures, at least at the limits utilised in the present study $\left(5-25^{\circ} \mathrm{C}\right)$. Most ligninolytic fungi have growth optima below $24^{\circ} \mathrm{C}$ or below although some are much more thermotolerant (Humphrey \& Siggers 1933). However E. glandulosa likely has a growth optima of around $25^{\circ} \mathrm{C}$ but growth rate may be severely slowed at $5^{\circ} \mathrm{C}$ compared with even $15^{\circ} \mathrm{C}$, at least on artificial media (Boddy 1983). Other pioneer species such as Hypoxylon fragiforme and Vuilleminia comedens exhibit increased wood decay rates at higher temperatures up to approximately $27^{\circ} \mathrm{C}$ indicating that the pioneer community is unlikely to be particularly thermophilic (Hiscox et al. 2016a). Certainly the influence of stable and unstable conditions on ligninolytic metabolism deserves further attention. 
Stable temperatures are not the norm in natural communities which are subject to diurnal and seasonal fluctuations. However stable temperatures are maintained under experimental conditions when the objective is to examine the impact of temperature on interaction outcomes and metabolic responses (Hiscox et al. 2016a; Moody et al. 2018a; O'Leary 2018). The present study indicates that inferring realworld processes from the behaviours of experimental systems under stable laboratory conditions may not always be appropriate as those artificial conditions may produce interaction outcomes and metabolic responses. Inconsistencies may be introduced by the very conditions designed to examine the mechanisms and drivers of ecosystem function in nature. At the very least, the extent to which stable versus variable temperatures affect experimental outcomes in wood require closer examination. 


\section{Chapter 7 - Synthesis and future directions}

\subsection{Synthesis}

The work presented in this thesis adds substantially to current knowledge pertaining to the structure and function of pioneer ligninolytic communities. These important communities, whose structure and assembly history influence the assembly trajectory, structure and functioning of later successions (Leather et al. 2014; Ottosson et al. 2014; Hiscox et al. 2015a, 2016b), are associated almost exclusively with attached dead branches and have likely been largely ignored previously due to their inaccessibility. The central aim of this thesis was to explore the environmental, biotic and spatial factors that contribute to the structure and functioning of the system in order to shed light on some of the historical processes that may contribute to patterns and perturbations in community structure and functioning in better-studied CWD systems. As a consequence the work presented here has quantified, for the first time, the relative abundance and size of mycelial colonies produced in natural dead wood systems facilitating analyses to test for the effects of spatial auto-correlation and environmental filtering on the mycelial community. Untargeted metabolomics was applied to the natural wood decay system for the first time and, in combination with enzyme assays, produced a first glimpse of wood decomposition processes in the natural pioneer community. Untargeted metabolomics and proteomics were paired for the first time in wood decomposition systems and their utility for examining the influence of priority effects and abiotic perturbations on fungal metabolism was demonstrated.

This chapter revisits the primary thesis hypotheses outlined in Chapter 1 and synthesises the main findings of the experimental and observational work presented in Chapters 3-6 in the context of those hypotheses. The strengths and limitations in the design of the presented studies are considered and the future research directions needed to build on this thesis's main findings are discussed.

\subsubsection{Hypothesis 1: Pioneer ligninolytic community structure is shaped mainly by purely spatial effects rather than deterministic environmental processes}

The pioneer ligninolytic community has been demonstrated to develop and colonise whole, dead attached branches from propagules that were present latently in the functional sapwood. The abundance and distribution of genetically compatible propagules within the same branch leads to the rapid formation of large longitudinal colonies of pioneer fungi (Boddy \& Rayner 1983a; Boddy 2001; Parfitt et al. 2010; Boddy et al. 2017) which then contribute to the exclusion of more combative species associated with the secondary succession (O'Leary et al. 2018), although high water potential is also thought to play a substantial role (Boddy et al. 2017). The factors that determine propagule distribution and abundance within branches are not known but it in all likelihood they are largely passive processes (Rodriguez et al. 2009). This might lead to high stochasticity in the distribution of propagules within branches which 
would lead to the hypothesis that the presence of several genetically compatible propagules in the same branch is a result of spatial auto-correlation rather than of deterministic environmental factors. Once all territory in a newly dysfunctional branch has been colonised, pioneer fungi are assumed to "jostle for territory" (Boddy et al. 2017 pg. 170) rather than to regularly engage in replacement interactions, unless the stress profile changes to tip the favour towards the selective traits of one or more community members in which case some degree of loss and gain of territory between community members might take place. The extent to which this happens is not known and I addressed this knowledge gap by applying a novel surveying approach at the mycelial level to fungal residents of attached beech branches (Chapter 3).

A unique combination of RSS, isolation methods and DNA sequencing provided a quantification of the relative size of the decay regions produced by pioneer ligninolytic fungi within their natural communities. Partitioning the variation explained by within- and between-branch spatial factors and branch physical characteristics led to the rejection of hypothesis 1 as branch physical characteristics, particularly mass loss and diameter of the wood, over-rode spatial autocorrelation at all taxonomic and functional levels. Mass loss of the wood was the major determinant of variation in spatial configuration of fungi at the species and order level suggesting that, as wood becomes more degraded, the spatial configuration of fungi within the community may shift through partial-replacement interactions so that those better able to deal with the specific physical and chemical parameters of the partially degraded resource in a given state are associated with those parameters. At the extreme end of wood mass loss, there was evidence that the taxonomic and functional diversity of communities opens up and that species immigrations are facilitated through vectors such as invertebrate activity which may destabilise previous levels of coexistence. Diameter of the wood was the most important factor determining the relative distribution of ascomycetes and basidiomycetes within branches as well as in determining the spatial configuration of species affiliated with different functional levels. Thicker branch wood will buffer the community against rapid drying and temperature fluctuations. Thus, fungi belonging to the two different phyla and with different functional strategies may be differently adapted to dealing with these dynamics.

\subsubsection{Hypothesis 2: The species richness of naturally formed pioneer communities increases with wood volume}

Positive relationships between species richness and wood volume or surface area in dead wood systems are known to exist in CWD (Bader et al. 1995; Renvall 1995; Lindblad 1998; Kruys \& Jonsson 1999; Heilmann-Clausen \& Christensen 2004). However, all studies reporting an SAR in wood decay systems to date have relied on sporocarp surveys from which the true species richness of mycelia, which are the drivers of wood decomposition processes, is impossible to determine in the short-term (Straatsma et al. 2001). Assuming that propagules of the pioneer ligninolytic community disperse within and between Page | 110 
branches due to passive processes (Rodriguez et al. 2009) it was hypothesised that bigger branches would harbour a greater number of propagules and therefore lead to the development of mycelial communities with greater species richness. Testing of this hypothesis was supported by isolation methods and DNA sequencing which determined that neither species richness nor diversity (1-D) correlated with either the volume or lateral surface area of the branches surveyed and thus to the rejection of hypothesis 2 (Chapter 3). This does not rule out the hypothesis that bigger branches harbour endophytic communities with greater abundance or diversity. However, it does suggest that the diversity of these pioneer communities may be driven by processes that override the SAR. The likely explanation for this might be that in newly dysfunctional, the unfavourable conditions brought about by high water potential leads to relative homogeneity of microhabitats such that habitat heterogeneity doesn't increase with the volume of wood. Therefore, access to territory for the newly-forming mycelial colonies is simply a race for space: those with the greatest number of genetically compatible propagules and the quickest growth habit will win out in smaller and larger branches. Once community establishment is complete, the relative size of colonies and the requirement to defend territory in three dimensions whilst also expending sufficient energy to decompose the lignocellulose resource and cope with abiotic stress likely maintains species coexistence (Hiscox et al. 2016a, 2017; O'Leary et al. 2018). Interestingly, while community structure changed with increasing mass loss, community diversity did not exhibit a correlation with mass loss. This lends further credence to the idea postulated above (Section 7.1.1) that community change over the degradation gradient and therefore over time may be in the form of spatial shifting of colonies, congruent with Boddy's (2017) description of pioneers jostling with each other for space.

\subsubsection{Hypothesis 3: Metabolic processes in the primary decay community are linked to biotic interactions between species, to variable abiotic environment and with the gradient of decomposition}

Variable functioning in ligninolytic communities has been linked to various biotic (Boddy 2000; Peiris $e t$ al. 2008; Hiscox et al. 2010a, 2017; Luo et al. 2017) and abiotic (A'Bear et al. 2012; Hiscox et al. 2016a; Moody et al. 2018b) factors mainly from the perspective of secondary successional communities and species. Community functioning and the factors that may determine community function in pioneer wood decay communities have largely been ignored. However recent work has shown that alterations to community configuration, substrate complexity and abiotic conditions can promote replacement interactions between pioneer species (Hiscox et al. 2016a) and may increase defensive ability (Hiscox et al. 2017; O'Leary et al. 2018). Laccase activity increased with increasing mass loss of natural attached branches, decreased with increasing water availability and covaried linearly with changes in the fungal metabolome (Chapter 4). The activity of several hydrolases related to the acquisition of limiting nutrients correlated negatively with wood mass loss indicating that in natural communities the nutrient 
profile may change drastically while wood is attached to standing trees and that the pioneer community might invest more highly in nutrient acquisition early on (Chapter 4). The relative concentration of storage compounds (e.g. various fatty acids) did not correlate with increasing loss of dry mass however their levels over the decay gradient may be stabilised due to the net effect of supply and demand as the nutrient profile alters (Chapter 4). There was substantial evidence of acidification of the wood environment through the production of several organic acids in both natural woodland communities (Chapter 4) and following biotic and abiotic interactions in model systems (Chapters 4, 5 and 6).

Variable temperature affected the metabolism of a stress tolerant pioneer, Exidia glandulosa, and a common constituent of pioneer communities in beech branches, Oudemansiella mucida (Chapter 6) at both the protein and metabolite levels. There was considerable evidence that in O. mucida, enhanced investment in lignocellulose decomposition may be promoted through variable temperature particularly due to the increased abundance of several CAZymes and monomeric constituents of lignin. E. glandulosa was not differently affected at the protein level by stable or variable temperature but a number of compounds were present in greater concentration under the stable regimen which may be associated with the species' stress-tolerance and indicate that E. glandulosa is less capable of dealing with stable conditions.

In recent years the influence of priority effects on community assembly trajectories of later successional stages has gathered interest (Fukami et al. 2010; Ottosson et al. 2014; Hiscox et al. 2015a, 2016b) and the order of arrival of members of the pioneer community, in addition to their identity and relative abundance, may be important (Frisch \& Green 2007). A clear priority effect was exerted on the metabolism of E. glandulosa following replacement interactions with either O. mucida or Stereum rugosum (Chapter 5). Following replacement of S. rugosum there was evidence of enhanced protein turnover and degradation which may have been driven by a change in nutrient acquisition strategy. These biotic interactions exerted a greater effect on the metabolic response of E. glandulosa than manipulation of the abiotic environment (Chapter 6) indicating that biotic interactions may play a more significant role in pioneer community functioning than previously thought.

\subsection{Strengths and limitations of experimental designs}

This thesis has resulted in a new measure of fungal abundance and the relative size of fungal colonies and territory held within woody substrata which may be more relevant to woodland ecosystem functioning, given that it quantifies fungi at the mycelial level, than measures previously used. As such the first quantitative data pertaining to mycelia in natural woody substrata were generated, allowing for the examination of spatial and environmental parameters on the spatial configurations, structure and diversity of naturally occurring wood decay communities. Fungal abundance relates not only to whole colonies, but also to their individual cellular components which are capable of autonomous survival and 
growth. However repeated surveying of the same colony introduces unavoidable pseudo-replication into the study presented (Chapter 3). Furthermore, while every care was taken to preserve spatial data within and between branches to account as far as possible for spatial auto-correlation, sections of wood from the same branch with similar mass loss values will have been available for roughly the same length of time. As such, the introduction of pseudo-replication was countered by introducing as much randomness into the sampling design as possible. The relevance of results presented here (Chapter 3 ) to pioneer wood decay communities in general cannot be rigorously interpreted in the absence of additional studies applying comparable surveying methods across various spatial scales however. That said, these results will help inform experimental work to examine species coexistence within pioneer communities and the effect that their natural structure has on community assembly trajectories and ecosystem functioning.

The utility of combining enzyme assays, and untargeted multi-omics to examine the metabolic responses of ligninolytic fungi in woody substrata has been proven (Chapters 4, 5 and 6). This thesis presents, for the first time, a glimpse of fungal metabolic responses at the metabolome level from environmental wood samples. Proteomics and metabolomics were successfully applied to examine the lasting impacts of priority effects on fungal metabolism following completion of replacement interactions by the latearriving species. The time-scale examined here however is still relatively short to that which must be maintained for a priority effect on later successional stages and it will be exciting to build on the preliminary success of applying these approaches to the study of priority effects and community assembly history.

\subsection{Future research directions}

With the discovery that pioneer wood decay communities are shaped, not only through spatial autocorrelation and passive dispersal mechanisms, but also changing environmental gradients, additional emphasis should be placed on examining interactive and metabolic responses of these forgotten engineers of woodland ecosystem processes. To build on the advances made in Chapter 4, which examined enzyme activities and the metabolome of ligninolytic fungi in their natural environment, full use should be made of these and other 'omic approaches to fully understand how vital ecosystem services are metabolically mediated by ligninolytic fungi in real world systems. Closer examination should also be undertaken into how pioneer ligninolytic species effect the first stages of wood resource decomposition and how the metabolic strategy of the pioneer community changes over time and in response to abiotic and biotic stimuli in model wood systems. Combining the results of multi-omics approaches to examine these dynamics could provide new biotechnological solutions particularly in the fields of biorefining and novel antifungal discovery. There is a clear need for enhanced characterisation of the expressed metabolome of ligninolytic fungi in wood and the transparent publication of large 'omics datasets is vital to this end. 


\subsection{Conclusions}

The distribution, community structure and metabolic responses of pioneer wood decay fungi, which contribute substantially to the overall decomposition of dead wood resource in natural systems, are affected by a range of biotic and abiotic factors. The structure and spatial configuration of natural communities displays characteristics commensurate with the effects of environmental filtering. Community diversity and initial community establishment is likely driven by a race for space however, perhaps due to a homogeneity in the availability of microhabitats and extensive niche-overlap. Biotic interactions between pioneer wood decay fungi may play a more significant role in community functioning than previously thought, exerting substantial influence on the nutrient acquisition strategies employed by competitively successful fungi. Environmental fluctuations also drive differential metabolic response but affect species with different ecological strategies differently. The metabolic responses of wood decay fungi influence wood decomposition rates and therefore the rate of nutrient turnover and the release of organic carbon in woodland systems. 


\section{References}

A'Bear, A.D., Boddy, L. \& Hefin Jones, T. (2012). Impacts of elevated temperature on the growth and functioning of decomposer fungi are influenced by grazing collembola. Glob. Chang. Biol., 18, 18231832.

A'Bear, A.D., Jones, T.H. \& Boddy, L. (2014a). Size matters: What have we learnt from microcosm studies ofdecomposer fungus-invertebrate interactions? Soil Biol. Biochem., 78, 274-283.

A'Bear, D.A., Jones, H.T., Kandeler, E. \& Boddy, L. (2014b). Interactive effects of temperature and soil moisture on fungal- mediated wood decomposition and extracellular enzyme activity, 70, 151-158.

Agilent Technologies Ltd. (2011). Environmental MSD chemStation E.02.02.1431.

Allen, M., Vandyke, J. \& Caceres, C. (2011). Metacommunity assembly and sorting in newly formed lake communities. Ecology, 92, 269-275.

Allen, R., Buchanan, P., Clinton, P. \& Cone, A. (2000). Composition and diversity of fungi on decaying logs in a New Zealand temperate beech (Northofagus) forest. Can. J. For. Res., 30, 1025-1033.

Anderson, M., Gorley, R.N. \& Clarke, K.R. (2008a). PERMANOVA+ for PRIMER: guide to software and statistical methods. Quest Research Ltd., Auckland, New Zealand.

Anderson, M., Gorley, R.N. \& Clarke, K.R. (2008b). PERMANOVA + for PRIMER: by. Guid. to Softw. Stat. Methods, 1.

Anderson, M. \& Legendre, P. (1998). An empirical comparison of permutation methods for tests of partial regression coefficients in a linear model. J. Stat. Comput. Simul., 62, 271-303.

Anke, T. (1995). The antifungal strobilurins and their possible ecological role. Can. J. Bot., 73, 940-945.

Anke, T., Hecht, H.J., Schramm, G. \& Steglich, W. (1979). Antibiotics from Basidiomycetes IX. Oudemansin, an antifungal antibiotic from Oudemansiella mucida (Schrader ex Fr.) Hoehnel (Agaricales). J. Antibiot. (Tokyo)., 32, 1112-1117.

Arakane, Y., Muthukrishnan, S., Beeman, R., Kanost, M. \& Kramer, K. (2005). Laccase 2 is the phenoloxidase gene required for beetle cuticle tanning. PNAS, 102, 11337-11342.

Arnstadt, T., Hoppe, B., Kahl, T., Kellner, H., Krüger, D., Bässler, C., et al. (2016). Patterns of laccase and peroxidases in coarse woody debris of Fagus sylvatica, Picea abies and Pinus sylvestris and their relation to different wood parameters. Eur. J. For. Res., 135, 109-124.

Audet, C., Macphee, S. \& Keller, W. (2013). Constructed ponds colonised by crustacean zooplankton: local and regional influences. Journbal Limnol., 72, 524-530. 
Bader, P., Jansson, S. \& Jonsson, B.G. (1995). Wood-inhabiting fungi and substratum decline in selectively logged boreal spruce forests. Biol. Conserv., 72, 355-362.

Báldi, A. (2008). Habitat heterogeneity overrides the species - area relationship. J. Biogeogr., 35, 675-681.

Baldrian, P. (2004). Increase of laccase activity during interspecific interactions of white-rot fungi. FEMS Microbiol. Ecol., 50, 245-253.

Baldrian, P. (2006). Fungal laccases-occurrence and properties. FEMS Microbiol. Rev., 30, 215-242.

Baldrian, P. (2008). Enzymes of saprotrophic basidiomycetes. In: The Ecology of Saprotrophic Basidiomycetes (eds. Boddy, L., Frankland, J. \& van West, P.). Academic Press, London, UK, pp. 20-41.

Baldrian, P. (2009). Microbial enzyme-catalyzed processes in soils and their analysis. Plant, Soil Environ., $55,370-378$.

Barrasa, J. (2014). Oudemansiella mucida CBS 558.79 Standard Draft (Project ID: 1056768 ). In: Study of the lignocellulolytic machinery in saprobic wood and leaflitter degrading Agaricales.

Bartlett, D.W., Clough, J.M., Godwin, J.R., Hall, A.A., Hamer, M. \& Parr-dobrzanski, B. (2002). The strobilurin fungicides. Pest Manag. Sci., 58, 648-662.

Beisner, B.E., Peres-neto, P.R., Lindström, E.S. \& Barnett, A. (2006). The role of environmental and spatial processes in structuring lake communities from bacteria to fish. Ecology, 87, 2985-2991.

Benjamini, Y. \& Hochberg, Y. (1995). Controlling the false discovery rate - a practical and powerful approach to multiple testing. J. R. Stat. Soc. Ser. B Methodol., 57, 289-300.

Bhattacharyya, S., Yu, H., Mim, C. \& Matouscheck, A. (2014). Regulated protein turnover: snapshots of the proteasome in action. Nat. Rev. Mol. Cell Biol., 15, 122-133.

Blakely, T.J. \& Didham, R.K. (2010). Disentangling the mechanistic drivers of ecosystem size-effects on species diversity. J. Anim. Ecol., 79, 1204-1214.

Boddy, L. (1983). Effect of temperature and water potential on growth rate of wood-rotting basidiomycetes. Trans. Br. Mycol. Soc., 80, 141-149.

Boddy, L. (2000). Interspecific combative interactions between wood-decaying basidiomycetes. FEMS Microbiol. Ecol., 31, 185-194.

Boddy, L. (2001). Fungal community ecology and wood decomposition processes in angiosperms: from standing tree to complete decay of coarse woody debris. In: Ecological Bulletins No. 49, Ecology of Woody Debris in Boreal Forests (eds. Jonsson, B.G. \& Kruys, N.). Oikos Editorial Office, pp. 43-56.

Boddy, L., Bardsley, D.W. \& Gibbon, O.M. (1987). Fungal Communities in Attached Ash Branches. New 
Phytol., 107, 143-154.

Boddy, L. \& Griffith, G.S. (1989). Role of endophytes and latent invasion in the development of decay communities in sapwood of angiospermous trees. Sydowia, 41, 41-73.

Boddy, L. \& Heilmann-Clausen, J. (2008). Basidiomycete community development in temperate angiosperm wood. In: Ecology of saprotrophic basidiomycetes (eds. Bodd, L., Frankland, J. \& van West, P.). Academic Press, London, UK, pp. 211-237.

Boddy, L., Hiscox, J., Gilmartin, E., Johnston, S. \& Heilmann-Clausen, J. (2017). Decay communities in angiosperm wood. In: The Fungal Community (eds. Dighton, J. \& White, J.). CRC Press, pp. 169-189.

Boddy, L. \& Rayner, A. (1983a). Ecological Roles of Basidiomycetes Forming Decay Communities in Attached Oak Branches. New Phytol., 93, 77-88.

Boddy, L. \& Rayner, A.D.M. (1983b). Origins of decay in living deciduous trees: the tole of moisture content and a re-appraisal of the expanded concept of tree decay. New Phytol., 94, 623-641.

Boddy, L. \& Swift, M. (1983). Wood decomposition in an abandoned beech and oak coppiced woodland in SE England. I. Patterns of wood-litter fall. Ecography (Cop.)., 6, 320-332.

Bohren, K.M., Bullock, B., Wermuths, B. \& Gabbay, K.H. (1989). The aldo-keto reductase superfamily. J. Biol. Chem., 264, 9547-9551.

Borcard, D., Legendre, P. \& Drapeau, P. (1992). Partialling out the spatial component of ecological variation. Ecology, 73, 1045-1055.

Bradford, M.A., Warren, R.J., Baldrian, P., Crowther, T.W., Maynard, D.S., Oldfield, E.E., et al. (2014). Climate fails to predict wood decomposition at regional scales. Nat. Clim. Chang. Lett., 4, 625-630.

Brakhage, A.A. (2013). Regulation of fungal secondary metabolism. Nat. Rev. Microbiol., 11, 21-32.

Brakhage, A.A. \& Schroeckh, V. (2011). Fungal secondary metabolites - strategies to activate silent gene clusters. Fungal Genet. Biol., 48, 15-22.

Breddam, K. (1986). Serine carboxypeptidases. A review. Carlsberg Res. Commun., 51, 83-128.

Carlsen, T., Aas, A., Lindner, D., Vrålstad, T., Schumacher, T. \& Kauserud, H. (2012). Don't make a mista(g)ke: is tag switching an overlooked source of error in amplicon pyrosequencing studies? Fungal Ecol., 5, 747-749.

Cartwright, K.S.G. \& Findlay, W.P.K. (1958). Decay of timber and its prevention. London.

Chapela, I. \& Boddy, L. (1988a). Fungal colonization of attached beech branches I. Early stages of development of fungal communities. New Phytol., 110, 39-45. 
Chapela, I.H. \& Boddy, L. (1988b). Fungal colonization of attached beech branches II. Spatial and temporal organization of communities arising from latent invaders in bark and functional sapwood, under different moisture regimes. New Phytol., 110, 47-57.

Chauhan, P.S., Goradia, B. \& Saxena, A. (2017). Bacterial laccase: recent update on production, properties and industrial applications. 3 Biotech, 7, 1-20.

Chong, J., Soufan, O., Li, C., Caraus, I., Li, S., Bourque, D., et al. (2018). MetaboAnalyst 4.0: towards more transparent and integrative metabolomics analysis. Nucleic Acids Res., 46, W486-W494.

Clarke, K., Tweedley, J. \& Valesini, F. (2014). Simple shade plots aid better long-term choices of data pretreatment in multivariate assemblage studies. J. Mar. Biol. Assoc. United Kingdom, 94, 1-16.

Clarke, K.R. \& Gorley, R.N. (2015). PRIMER v7: user manual / tutorial. Plymouth, NZ.

Cline, L.C., Schilling, J.S., Menke, J., Groenhof, E. \& Kennedy, P.G. (2018). Ecological and functional effects of fungal endophytes on wood decomposition, 181-191.

Cline, L.C. \& Zak, D.R. (2015). Initial colonization, community assembly and ecosystem function: Fungal colonist traits and litter biochemistry mediate decay rate. Mol. Ecol., 24, 5045-5058.

Collins, C. (2013). A genomic and proteomic investigation of the plant pathogen Armillaria mellea: buried treasure or hidden threat? PhD Thesis. NUI Maynooth.

Conesa, A., Götz, S., Garcia-Gomez, J., Terol, J., Talon, M. \& Robles, M. (2005). Blast2GO: a universal tool for annotation, visualization and analysis in functional genomics research. Bioinformatics, 21, 36743676.

Converti, A., Perego, P. \& Domínguez, J. (1999). Xylitol production from hardwood hemicellulose hydrolysates by Pachysolen tannophilus, Debaryomyces hansenii and Candida guilliermondii. Appl. Biochem. Biotechnol., 82, 141-151.

Cox, J. \& Mann, M. (2008). MaxQuant enables high peptide identification rates, individualized ppbrange mass accuracies and proteome-wide protein quantification. Nat. Biotechnol., 26, 1367-1372.

Criquet, S., Farnet, A.M., Tagger, S. \& Petit, J. Le. (2000). Annual variations of phenoloxidase activities in an evergreen oak litter: influence of certain biotic and abiotic factors. Soil Biol. Biochem. Biochem., 32, 1505-1513.

Crowther, T.W., Littleboy, A., Jones, T.H. \& Boddy, L. (2012). Interactive effects of warming and invertebrate grazing on the outcomes of competitive fungal interactions. FEMS Microbiol. Ecol., 81, $419-426$.

D’Annibale, A., Ricci, M., Leonardi, V., Quaratino, D., Mincione, E. \& Petruccioli, M. (2005). Degradation Page | 118 
of aromatic hydrocarbons by white-rot fungi in a historically contaminated soil. Biotechnol. Bioeng., $90,723-731$.

D'Arcy, P. \& Mallard, W.G. (2004). Automated Mass Spectral Deconvolution Idnetification System AMDIS user guide. National Institute of Standards and Technology.

Dawson-Andoh, B. \& Morell, J. (1992). Extraction of proteins from wood wafers colonized by decay fungi. Holfzforschung, 46, 117-120.

Debeljak, M. (2006). Coarse wood debris in virgin and managed forest. Ecol. Indic., 6, 733-742.

Deveau, A., Kohler, A. \& Martin, F. (2008). The major pathways of carbohydrate metabolism in the ectomycorrhizal basidiomycete Laccaria bicolor S238N. New Phytol., 180, 379-390.

Dhami, M.K., Hartwig, T. \& Fukami, T. (2016). Genetic basis of priority effects: Insights from nectar yeast. Proc. R. Soc. B Biol. Sci., 283.

Dickie, I.A., Allen, R.B., Buchanan, P.K., Fukami, T., Wilkie, J.P., Allen, R.B., et al. (2011). Do assembly history effects attenuate from species to ecosystem properties? A field test with wood-inhabiting fungi. Ecol. Lett., 15, 133-141.

Doddapaneni, H., Subramanian, V., Fu, B. \& Cullen, D. (2013). A comparative genomic analysis of the oxidative enzymes potentially involved in lignin degradation by Agaricus bisporus. Fungal Genet. Biol., 55, 22-31.

Dolan, S., Owens, R., O’Keeffe, G., Hammel, S., Fitzpatrick, D., Jones, G., et al. (2014). Regulation of nonribosomal peptide synthesis: bis-thiomethylation attenuates gliotoxin biosynthesis in Aspergillus fumigatus. Chem. Biol., 21, 999-1012.

Eastwood, D.C., Floudas, D., Binder, M., Majcherczyk, A., Schneider, P., Aerts, A., et al. (2011). The plant cell wall-decomposing machinery underlies the functional diversity of forest fungi. Science (80-. )., $333,762-765$.

Elleuche, S. \& Poggeler, S. (2009). Evolution of carbonic anhydrases in fungi. Curr. Genet., 55, 211-222.

Elleuche, S. \& Pöggeler, S. (2010). Carbonic anhydrases in fungi. Microbiology, 156, 23-29.

Eriksson, K., Blanchette, R. \& Ander, P. (1990). Biodegradation of lignin. In: Microbial and enzymatic degradation of wood and wood components (eds. Eriksson, K., Blanchette, R. \& Ander, P.). SpringerVerlag, Berlin, Germany, pp. 225-333.

Eyre, C., Muftah, W., Hiscox, J., Hunt, J., Kille, P., Boddy, L., et al. (2010). Microarray analysis of differential gene expression elicited in Trametes versicolor during interspecific mycelial interactions. Fungal Biol., 114, 646-660. 
Falconer, R.E., Bown, J., White, N. \& Crawford, J. (2011). Linking individual behaviour to community scale patterns in fungi. Fungal Ecol., 4, 76-82.

Finlay, B. (2002). Global dispersal of free-living microbial eukaryote species. Science (80-. )., 296, 10611063.

Flory, M., Angeles, L. \& Cen-, C.M. (2007). SpectConnect for metabolomics. J. Proteome Res., 6, 435-437.

Floudas, D., Binder, M., Riley, R., Barry, K., Blanchette, R., Henrissat, B., et al. (2012). Paleozoic origin of enzymatic from 31 fungal genomes. Science (80-. )., 336, 1715-1719.

Forest Europe. (2012). The Ministerial Conference on the protection of forests in Europe. Available at: https://www.eea.europa.eu/data-and-maps/indicators/forest-deadwood-1/assessment\%0A1\#tab-related-briefings. Last accessed 25 November 2018.

Freedman, D. \& Lane, D. (1983). A nonstochastic interpretation of reported significance levels. J. Bus. Econ. Stat., 1, 292-298.

Freitag, M. \& JJ, M. (1992). Changes in selected enzyme activities during growth of pure and mixed cultures of the white-rot decay fungus Trametes versicolor and the potential biocontrol fungus Trichoderma harzianum. Can. J. Microbiol., 38, 317-323.

Freschert, G.T., Weedon, J.T., Aerts, R., van Hal, J.R. \& Cornelissen, J.H.C. (2014). Interspecific differences in wood decay rates: insights from a new short-term method to study long-term wood decomposition. J. Ecol., 100, 161-170.

Frisch, D. \& Green, A. (2007). Copepods come first: rapid colonization of new temporary ponds. Fundam. Appl. Limnol., 168, 289-297.

Frolkis, A., Knox, C., Lim, E., Jewison, T., Law, V., Hau, D.D., et al. (2009). SMPDB: The small molecule pathway database. Nucleic Acids Res., 38, 480-487.

Fukami, T. (2015). Historical contingency in community assembly: integrating niches, species pools, and priority effects. Annu. Rev. Ecol. Evol. Syst., 46, 1-23.

Fukami, T., Ian, A., Wilkie, J.P., Paulus, C., Park, D. \& Roberts, A. (2010). Assembly history dictates ecosystem functioning: evidence from wood decomposer communities. Ecol. Lett., 13, 675-684.

Fukami, T. \& Morin, P.J. (2003). Productivity-biodiversity relationships depend on the history of community assembly. Nature, 424, 423-426.

Fukuzumi, T. (1960). Enzymatic degradation of lignin Part I. Paperchromatographical separation of intermediate products of lignin by the wood-rotting fungus Poria subacida (Peck) Sacc. J. Agric. Chem. Soc. Japan, 24, 728-736.

Page | 120 
Gene Ontology Consortium. (2004). The Gene Ontology (GO) database and informatics resource. Nucleic Acids Res., 32, D258-D261.

Ghoreishi, S.M. \& Shahrestani, R.G. (2009). Innovative strategies for engineering mannitol production. Trends Food Sci. Technol., 20, 263-270.

Gleason, H.A. (1927). Further views on the succession-concept. Ecology, 8, 299-326.

Goodell, B., Jellison, J., Liu, J., Daniel, G., Paszczynski, A., Fekete, F., et al. (1997). Low molecular weight chelators and phenolic compounds isolated from wood decay fungi and their role in the fungal biodegradation of wood. J. Biotechnol., 53, 133-162.

Götz, S., García-Gómez, J., Williams, T., Nagaraj, S., Nueda, M., Robles, M., et al. (2008). Highthroughput functional annotation and data mining with the Blast2GO suite. Nucleic Acids Res., 36, 3420-3435.

Hanson, C.A., Fuhrman, J.A., Horner-Devine, M.C. \& Martiny, J.B.H. (2012). Beyond biogeographic patterns: processes shaping the microbial landscape. Nat. Rev. Microbiol., 10, 497-506.

Harmon, M.E., Franklin, J.F., Swanson, F.J., Sollins, P., Gregory, S. V., Lattin, J.D., et al. (1986). Ecology of coarse woody debris in temperate ecosystems. Adv. Ecol. Res., 15, 135-305.

Harold, S., Tordoff, G.M., Jones, T.H. \& Boddy, L. (2005). Mycelial responses of Hypholoma fasciculare to collembola grazing: effect of inoculum age, nutrient status and resource quality. Mycol. Res., 109, 927-935.

Hasenauer, H. (1997). Dimensional relationships of open-grown trees in Austria. For. Ecol. Manage., 96, 197-206.

Heilmann-Clausen, J. (2001). A gradient analysis of communities of macrofungi and slime moulds on decaying beech logs. Mycol. Res., 105, 575-596.

Heilmann-Clausen, J., Aude, E., van Dort, K., Christensen, M., Piltaver, A., Veerkamp, M., et al. (2014). Communities of wood-inhabiting bryophytes and fungi on dead beech logs in Europe - reflecting substrate quality or shaped by climate and forest conditions? J. Biogeogr., 41, 2269-2282.

Heilmann-Clausen, J. \& Boddy, L. (2005). Inhibition and stimulation effects in communities of wood decay fungi: exudates from colonized wood influence growth by other species. Microb. Ecol., 49, 399-406.

Heilmann-Clausen, J. \& Boddy, L. (2008). Distribution patterns of wood-decay basidiomycetes at the landscape to global scale. In: The Ecology of Saprotrophic Basidiomycetes (eds. Boddy, L., Frankland, J.C. \& van West, P.). British Mycological Society Symposia Series, pp. 263-275. 
Heilmann-Clausen, J. \& Christensen, M. (2004). Does size matter? On the importance of various dead wood fractions for fungal diversity in Danish beech forests. For. Ecol. Manage., 201, 105-117.

Hein, S. \& Spiecker, H. (2007). Comparative analysis of occluded branch characteristics for Fraxinus excelsior and Acer pseudoplatanus with natural and artificial pruning. Can. J. For. Res., 37, 14141426.

Hendry, S.J., Boddy, L. \& Lonsdale, D. (2002). Abiotic variables effect differential expression of latent infections in beech (Fagus sylvatica). New Phytol., 155, 449-460.

Hendry, S.J., Lonsdale, D. \& Boddy, L. (1998). Strip-cankering of beech (Fagus sylvatica): Pathology and distribution of symptomatic trees. New Phytol., 140, 549-565.

Henriksson, G., Johansson, G. \& Pettersson, G. (2000). A critical review of cellobiose dehydrogenases. J. Biotechnol., 78, 93-113.

Hibbett, D.S., Binder, M., Bischoff, J.F., Blackwell, M., Cannon, F., Eriksson, O.E., et al. (2007). A higherlevel phylogenetic classification of the Fungi, 111, 509-547.

Hibbett, D.S., Donoghue, M.J. \& Olmstead, R. (2001). Analysis of Character Correlations Among Wood Decay Mechanisms, Mating Systems, and Substrate Ranges in Homobasidiomycetes. Syst. Biol., 50, 215-242.

Higuchi, T. (1997). Biochemistry and molecular biology of wood. Springer-Verlag, Londond, UK.

Hirst, J.E. (1995). The ecology and physiology of endophytes of angiosperm stems. Thesis (Ph.D.). Cardiff University, Cardiff.

Hiscox, J., Baldrian, P., Rogers, H.J. \& Boddy, L. (2010a). Changes in oxidative enzyme activity during interspecific mycelial interactions involving the white-rot fungus Trametes versicolor. Fungal Genet. Biol., 47, 562-571.

Hiscox, J. \& Boddy, L. (2017). Armed and dangerous: chemical warfare in wood decay communities. Fungal Biol. Rev., 31, 169-184.

Hiscox, J., Clarkson, G., Savoury, M., Powell, G., Savva, I., Lloyd, M., et al. (2016a). Effects of precolonisation and temperature on interspeci fi c fungal interactions in wood. Fungal Ecol., 21, 32-42.

Hiscox, J., Hibbert, C., Rogers, H.J. \& Boddy, L. (2010b). Monokaryons and dikaryons of Trametes versicolor have similar combative, enzyme and decay ability. Fungal Ecol., 3, 347-356.

Hiscox, J., Leary, J.O. \& Boddy, L. (2018). Fungus wars: basidiomycete battles in wood decay. Stud. Mycol., 89, 117-124.

Hiscox, J., Savoury, M., Johnston, S.R., Parfitt, D., Müller, C.T., Rogers, H.J., et al. (2016b). Location , Page | 122 
location , location: priority effects in wood decay communities may vary between sites, 18, 19541969.

Hiscox, J., Savoury, M., Müller, C.T., Lindahl, B.D., Rogers, H.J. \& Boddy, L. (2015a). Priority effects during fungal community establishment in beech wood. ISME J., 9, 2246-2260.

Hiscox, J., Savoury, M., Toledo, S., Kingscott-edmunds, J., Bettridge, A., Waili, N. Al, et al. (2017). Threesomes destabilise certain relationships: multispecies interactions between wood decay fungi in natural resources. FEMS Microbiol. Ecol., 93, 1-11.

Hiscox, J., Savoury, M., Vaughan, I.P., Müller, C.T. \& Boddy, L. (2015b). Antagonistic fungal interactions influence carbon dioxide evolution from decomposing wood. Fungal Ecol., 4, 24-32.

Hofrichter, M., Ullrich, R., Pecyna, M., Liers, C. \& Lundell, T. (2010). New and classic families of secreted fungal heme peroxidases. Appl. Microbiol. Biotechnol., 87, 871-897.

Hoppe, B., Purahong, W., Wubet, T., Kahl, T., Bauhus, J., Arnstadt, T., et al. (2016). Linking molecular deadwood-inhabiting fungal diversity and community dynamics to ecosystem functions and processes in Central European forests. Fungal Divers., 33, 367-379.

Hori, C., Gaskell, J., Igarashi, K., Kersten, P., Mozuch, M., Samejima, M., et al. (2014). Temporal alterations in the secretome of the selective ligninolytic fungus ceriporiopsis subvermispora during growth on aspen wood reveal this organism's strategy for degrading lignocellulose. Appl. Environ. Microbiol., 80, 2062-2070.

Hummel, J., Strehmel, N., Selbig, J., Walther, D. \& Kopka, J. (2010). Decision tree supported substructure prediction of metabolites from GC-MS profiles. Metabolomics, 6, 322-333.

Humphrey, C.J. \& Siggers, P. V. (1933). Temperature relations of wood-destroying fungi. J. agric. Res, 47, 997-1008.

Hurlbert, S. (1984). Pseudoreplication and the design of ecological field experiments. Ecol. Monogr., 54, 187-211.

Hutchinson, G. (1961). The paradox of the plankton. Am. Nat., 95, 137-145.

Iakovlev, A. \& Stenlid, J. (2000). Spatiotemporal Patterns of Laccase Activity in Interacting Mycelia of Wood-Decaying Basidiomycete Fungi. Microb. Ecol., 39, 236-245.

Jessup, C.M., Kassen, R., Forde, S.E., Kerr, B., Buckling, A., Rainey, P.B., et al. (2004). Big questions, small worlds: Microbial model systems in ecology. Trends Ecol. Evol., 19, 189-197.

Jones, P., Binns, D., Chang, H.Y., Fraser, M., Li, W., McAnulla, C., et al. (2014). InterProScan 5: genomescale protein function classification. Bioinformatics, 30, 1236-1240. 
Kahl, T., Arnstadt, T., Baber, K., Bässler, C., Bauhus, J., Borken, W., et al. (2017). Wood decay rates of 13 temperate tree species in relation to wood properties, enzyme activities and organismic diversities. For. Ecol. Manage., 391, 86-95.

Kanehisa, M. \& Goto, S. (2000). KEGG; Kyoto Encyclopedia of Genes and Genomes .pdf, 28, 27-30.

Kasson, M.T., Wickert, K.L., Stauder, C.M., Macias, A.M., Berger, M.C., Simmons, D.R., et al. (2016). Mutualism with aggressive wood-degrading Flavodon ambrosius (Polyporales) facilitates niche expansion and communal social structure in Ambrosiophilus ambrosia beetles. Fungal Ecol., 23, 8696.

Keddy, P. (1992). Assembly and response rules: two goals for predictive community ecology. J. Veg. Sci., 3, 157-164.

Keller, N.P., Turner, G. \& Bennett, J.W. (2005). Fungal secondary metabolism - From biochemistry to genomics. Nat. Rev. Microbiol., 3, 937-947.

Kennedy, P.G., Peay, K.G. \& Bruns, T.D. (2009). Root tip competition among ectomycorrhizal fungi: Are priority effects a rule or an exception? Ecology, 90, 2098-2107.

Kiziridis, D., Boddy, L., Eastwood, D.C., Yuan, C. \& Fowler, M.S. (2018). A mechanistic perspective of ecological networks highlights the contribution of alternative interaction strategies. bioRxiv 192260 [Preprint]. Available at: http://dx.doi.org/10.1101/192260. Last accessed 1 December 2018.

Kõljalg, U., Larsson, K., Abarenkov, K., Nilsson, R., Alexander, I., Eberhardt, U., et al. (2005). UNITE: a database providing web-based methods for the molecular identification of ectomycorrhizal fungi. New Phytol., 166, 1063-1068.

Kõljalg, U., Nilsson, R.H., Abarenkov, K., Tedersoo, L., Taylor, A.F.S., Bahram, M., et al. (2013). Towards a unified paradigm for sequence-based identification of fungi. Mol. Ecol., 22, 5271-5277.

Koonjul, P.K., Brandt, W.F., Farrant, J.M. \& Lindsey, G.G. (1999). Inclusion of polyvinylpyrrolidone in the polymerase chain reaction reverses the inhibitory effects of polyphenolic contamination of RNA. Nucleic Acids Res., 27, 915-916.

Kopka, J., Schauer, N., Krueger, S., Birkemeyer, C., Usadel, B., Bergmüller, E., et al. (2005). GMD@CSB.DB: The Golm metabolome database. Bioinformatics, 21, 1635-1638.

Kopra, K. \& Fyles, J. (2005). Woody debris and nutrient cycling: should we care? Res. Note Ser., 8.

Kraigher, H., Juurc, D., Kalan, P., Kutnar, L., Levanic, T., Rupel, M., et al. (2002). Beech coarse woody debris characteristics in two virgin forest reserves in southern slovenia. For. Wood Sci. Technol., 69, 91-134. 
Kramer, S., MArhan, S., Haslwimmer, H. \& Kendeler, E. (2013). Temporal variation of surface and subsoil abundance and function of the soil microbial community in an arable soil. Soil Biol. Biochem., $61,79-85$.

Krankina, O., Harmon, M. \& Griazkin, A. (1999). Nutrient stores and dynamics of woody detritus in a boreal forest: modeling potential implications at the stand level. Can. J. For. Res., 29, 20-32.

Kruys, N. \& Jonsson, B.G. (1999). Fine woody debris is important for species richness on logs in managed boreal spruce forests of northern Sweden. Can. J. For. Res., 29, 1295-1299.

Kubartová, A., Ottosson, E., Dahlberg, A., Stenlid, J., Ottosson, E., Dahlberg, A., et al. (2012). Patterns of fungal communities among and within decaying logs revealed by 454 sequencing. Mol. Ecol., 21, 4514-4532.

Kuuskeri, J., Häkkinen, M., Laine, P., Smolander, O.P., Tamene, F., Miettinen, S., et al. (2016). Biotechnology for biofuels time-scale dynamics of proteome and transcriptome of the white-rot fungus Phlebia radiata: growth on spruce wood and decay effect on lignocellulose. Biotechnol. Biofuels, 9, 1-22.

Larena, I., Salazar, O., Gonza, V., Julia, C. \& Rubio, V. (1999). Design of a primer for ribosomal DNA internal transcribed spacer with enhanced specificity for ascomycetes. J. Biotchnology, 75, 187-194.

Lassauce, A., Paillet, Y., Jactel, H. \& Bouget, C. (2011). Deadwood as a surrogate for forest biodiversity: Meta-analysis of correlations between deadwood volume and species richness of saproxylic organisms. Ecol. Indic., 11, 1027-1039.

Leather, S.R., Baumgart, E.A., Evans, H.F. \& Quicke, D.L.J. (2014). Seeing the trees for the wood - beech (Fagus sylvatica) decay fungal volatiles influence the structure of saproxylic beetle communities. Insect Conserv. Divers., 7, 314-326.

Legendre, P. (1993). Spatial autocorrelation: trouble or new paradigm? Ecology, 74, 1659-1673.

Legendre, P. (2008). Studying beta diversity: ecological variation partitioning by multiple regression and canonical analysis. J. Plant Ecol., 1, 3-8.

Legendre, P. \& Anderson, M. (1999). Distance-based redundancy analysis: testing multispecies responses in multifactorial ecological experiments. Ecol. Monogr., 69, 1-24.

Leonardi, R., Zhang, Y.M., Rock, C.O. \& Jackowski, S. (2005). Coenzyme A: back in action. Prog. Lipid Res., 44, 125-153.

Leopold, D.R., Paula, J., Dickie, I.A., Robert, B. \& Buchanan, P.K. (2017). Priority effects are interactively regulated by top-down and bottom-up forces: evidence from wood decomposer communities. Ecol. 
Lett., 20, 1054-1063.

Lindahl, B. \& Boberg, J. (2008). Distribution and function of litter basidiomycetes in coniferous forests. In: The Ecology of Saprotrophic Basidiomycetes. pp. 184-196.

Lindahl, B.D. \& Finlay, R.D. (2006). Activities of chitinolytic enzymes during primary and secondary colonization of wood by basidiomycetous fungi. New Phytol., 169, 389-397.

Lindblad, I. (1998). Wood-inhabiting fungi on fallen logs of Norway spruce: relations to forest management and substrate quality. Nord. J. Bot., 18, 243-255.

Linstrom, P. \& Mallard, W. (2001). NIST Chemistry WebBook. NIST Standard Reference Database No. 69.

Liu, C.M., Kachur, S., Dwan, M.G., Abraham, A.G., Aziz, M., Hsueh, P., et al. (2012). FungiQuant: a broad-coverage fungal quantitative real-time PCR assay. BMC Microbiol., 12, 1-11.

Lonsdale, D., Pautasso, M. \& Holdenrieder, O. (2008). Wood-decaying fungi in the forest: Conservation needs and management options. Eur. J. For. Res., 127, 1-22.

Louette, G. \& De Meester, L. (2007). Predation and priority effects in experimental zooplankton communities. Oikos, 116, 419-426.

Louette, G., de Meester, L. \& Declerck, S. (2008). Assembly of zooplankton communities in newly created ponds. Freshw. Biol., 53, 2309-2320.

Luckinbill, L. (1973). Coexistence in laboratory populations of Paramecium aurelia and its predator Didinium nastutum. Ecology, 54, 1320-1327.

Luo, F., Zhong, Z., Liu, L., Igarashi, Y., Xie, D. \& Li, N. (2017). Metabolomic differential analysis of interspecific interactions among white rot fungi Trametes versicolor, Dichomitus squalens and Pleurotus ostreatus. Sci. Rep., 7, 1-11.

Lynd, L.R., Weimer, P.J., Zyl, W.H. Van \& Pretorius, I.S. (2002). Microbialcellulose utilization: fundamentals and biotechnology. Microbiol. Mol. Biol. Rev., 66, 506-577.

Magan, N. (2008). Ecophysiology: impact of environment on growth, synthesis of compatible solutes and enzyme production. In: Ecology of Saprotrophic Basidiomycetes (eds. Boddy, L., Frankland, J. \& van West, P.). Academic Press, London, UK, pp. 63-78.

Magnuson, J.K. \& Lasure, L. (2004). Organic acid production by filamentous fungi. In: Advances in Fungal Biotechnology for Industry, Agriculture and Medicine (eds. Lange, J. \& Lange, L.). Kluwer Academic Press, Drodecht, NL, pp. 307-340.

Martiny, J.B.H., Bohannan, B.J.M., Brown, J., Colwell, R., Fuhrman, J., Green, J., et al. (2006). Microbial biogeography: putting microorganisms on the map. Nat. Rev. Microbiol., 4, 102-112.

Page | 126 
Mathieson, T., Franken, H., Kosinski, J., Kurzawa, N., Zinn, N., Sweetman, G., et al. (2018). Systematic analysis of protein turnover in primary cells. Nat. Commun., 9, 1-10.

Mathis, A.D., Naylor, B.C., Carson, R.H., Evans, E., Harwell, J., Knecht, J., et al. (2017). Mechanisms of in Vivo ribosome maintenance change in response to nutrient signals. Mol. Cell. Proteomics, 16, 243254.

Maynard, D.S., Bradford, M.A., Lindner, D.L., Van Diepen, L.T.A., Frey, S.D., Glaeser, J.A., et al. (2017). Diversity begets diversity in competition for space. Nat. Ecol. Evol., 1.

McArdle, B. \& Anderson, M. (2001). Fitting multivariate models to community data: a comment on distance-based redundancy analysis. Ecology, 82, 290-297.

Mittelbach, G.G., Steiner, C.F., Scheiner, S.M., Katherine, L., Reynolds, H.L., Waide, R.B., et al. (2001). What is the observed relationship between species richness and productivity? Ecology, 84, 33903395.

Miyauchi, S., Navarro, D., Grisel, S., Chevret, D., Berrin, J. \& Rosso, M. (2017). The integrative 'omics of white-rot fungus Pycnoporus coccineus reveals co-regulated CAZymes for orchestrated lignocellulose breakdown. PLoS One, 12, DOI:org/10.1371/journal.pone.0175528.

Moloney, N., Owens, R., Meleady, P., Henry, M., Dolan, S., Mulvihill, E., et al. (2016). The ironresponsive microsomal proteome of Aspergillus fumigatus. J. Proteomics, 136, 99-111.

Moody, S.C., Dudley, E., Hiscox, J. \& Boddy, L. (2018a). Interdependence of primary metabolism and xenobiotic mitigation characterizes the proteome of Bjerkandera adusta during wood decomposition. Appl. Environ. Microbiol., 84, 1-14.

Moody, S.C., Dudley, E., Hiscox, J., Boddy, L. \& Eastwood, D.C. (2018b). Interdependence of primary metabolism and xenobiotic mitigation characterizes the proteome of Bjerkandera adusta during wood decomposition. Appl. Environ. Microbiol., 84, 1-14.

Musrati, R.A., Kollárová, M., Mernik, N. \& Mikulásová, D. (1998). Malate dehydrogenase: distribution, function and properties. Gen. Physiol. Biophys., 17, 193-210.

Naeem, S. \& Li, S. (1997). Biodiversity enhances ecosystem reliability. Nature, 390, 507-509.

Nagy, L.G., Riley, R., Tritt, A., Adam, C., Daum, C., Floudas, D., et al. (2016). Comparative genomics of early-diverging mushroom-forming fungi provides insights into the origins of lignocellulose decay capabilities. Mol. Biol. Evol., 33, 959-970.

Nakamura, Y., Torikai, K., Ohto, Y., Murakami, a, Tanaka, T. \& Ohigashi, H. (2000). A simple phenolic antioxidant protocatechuic acid enhances tumor promotion and oxidative stress in female ICR 
mouse skin: dose-and timing-dependent enhancement and involvement of bioactivation by tyrosinase. Carcinogenesis, 21, 1899-907.

Nevell, T. \& Zeronian, S. (1985). Cellulose chemistry and its applications. Ellis Horwood, Chirchester, UK.

Nguyen, N., Smith, D., Peay, K. \& Kennedy, P. (2014). Parsing ecological signal from noise in next generation amplicon sequencing Letter Parsing ecological signal from noise in next generation amplicon sequencing. New Phytol., 205, 1389-1393.

Nguyen, N., Song, Z., Bates, S., Branco, S., Tedersoo, L., Menke, J., et al. (2016). FUNGuild: an open annotation tool for parsing fungal community datasets by ecological guild. Fungal Ecol., 20, 241-248.

Nilsson, R.H., Larsson, K., Taylor, A.F.S., Bengtsson-palme, J., Jeppesen, T.S., Schigel, D., et al. (2018). The UNITE databse for molecular identification of fungi: handling dark taxa and parallel taxanomic classifications. Nucleic Acids Res., 1-6.

Nimz, H. (1974). Beech lignin - proposal of a constitutional scheme. Angew. Chemie Int. Ed. English, 13, 313-321.

Noll, L., Leonhardt, S., Arnstadt, T., Hoppe, B., Poll, C., Matzner, E., et al. (2016). Forest ecology and management fungal biomass and extracellular enzyme activities in coarse woody debris of 13 tree species in the early phase of decomposition. For. Ecol. Manage., 378, 181-192.

O’Keeffe, G., Hammel, S., Owens, R., Keane, T., Fitzpatrick, D., Jones, G., et al. (2014). RNA-seq reveals the pan-transcriptomic impact of attenuating the gliotoxin self-protection mechanism in Aspergillus fumigatus. BMC Genomics, 15, 1.

O'Leary, J. (2018). Multi-dimensional mycelial interactions. Ph.D Thesis. Cardiff University.

O'Leary, J., Eastwood, D., Müller, C. \& Boddy, L. (2018). Emergent properties arising from spatial heterogeneity in $\mathrm{fl}$ uence fungal community dynamics. Fungal Ecol., 33, 32-39.

Ocón, A., Hampp, R. \& Requena, N. (2007). Trehalose turnover during abiotic stress in arbuscular mycorrhizal fungi. New Phytol., 174, 879-891.

Oksanen, L. (2003). Logic of experiments in ecology: is pseudoreplication a pseudoissue? Oikos, 94, 2738.

Oliver, A., Brown, S., Callaham, M. \& Jumpponen, A. (2015). Polymerase matters: non-proofreading enzymes inflate fungal community richness estimates by up to 15\%. Fungal Ecol., 15, 86-89.

Ottosson, E., Nordén, J., Dahlberg, A., Edman, M., Jönsson, M., Larsson, K.H., et al. (2014). Species associations during the succession of wood-inhabiting fungal communities. Fungal Ecol., 11, 17-28.

Ovaskainen, O., Nokso-koivisto, J., Hottola, J. \& Rajala, T. (2010). Identifying wood-inhabiting fungi Page | 128 
with 454 sequencing - what is the probability that BLAST gives the correct species?, 3, 274-283.

Owens, R., O'Keeffe, G., Smith, E., Dolna, S., Hammel, S., Sheridan, K., et al. (2015). Interplay between gliotoxin resistance, secretion, and the methyl/methionine cycle in Aspergillus fumigatus. Eukaryot. Cell, 14, 941-957.

Pan, Y., Birdsey, R.A., Fang, J., Houghton, R., Kauppi, P.E., Kurz, W.A., et al. (2011). A large and persistent carbon sink in the world's forests. Science (80-. )., 333, 988-993.

Parfitt, D., Hunt, J., Dockrell, D., Rogers, H.H.J., Boddy, L. \& Griffith, G.W. (2010). Do all trees carry the seeds of their own destruction? PCR reveals numerous wood decay fungi latently present in sapwood of a wide range of angiosperm trees. Fungal Ecol., 3, 338-346.

Peiris, D., Dunn, W.B., Brown, M., Kell, D.B., Roy, I. \& Hedger, J.N. (2008). Metabolite profiles of interacting mycelial fronts differ for pairings of the wood decay basidiomycete fungus, Stereum hirsutum with its competitors Coprinus micaceus and Coprinus disseminatus. Metabolomics, 4, 5262.

Pence, H. \& Williams, A. (2010). ChemSpider: an online chemical information resource. J. Chem. Educ., $87,1123-1124$.

Peres-Neto, P.R. \& Legendre, P. (2010). Estimating and controlling for spatial structure in the study of ecological communities. Glob. Ecol. Biogeogr., 19, 174-184.

Petchey, O., McPhearson, P., Casey, T. \& Morin, P. (1999). Environmental warming alters food-web structure and ecosystem function. Nature, 402, 69-72.

Pettersen, R. (1984). The chemical composition of wood. In: The chemistry of solid wood (ed. Rowell, R.). American Chemical Society, pp. 57-126.

Polis, G.A. \& Hurd, S.D. (1995). Extraordinarily high spider densities on islands - flow of energy from the marine to terrestrial food webs and the absence of predation. Proc. Natl. Acad. Sci. U. S. A., 92, 4382-4386.

Poll, C., Ingwersen, J., Stemmer, M., Gerzabek, M. \& Kandeler, E. (2006). Mechanisms of solute transport influence small-scale abundance and function of soil microorganisms at the soil-litter interface. Eur. Jounral Soil Sci., 57, 583-595.

Presley, G.N., Panisko, E., Purvine, S.O. \& Schilling, J.S. (2018). Coupling secretomics with enzyme activities to compare the temporal processes of wood metabolism among white and brown rot fungi. Appl. Environ. Microbiol., 84.

Pronk, J., Steensma, H. \& van Dijken, J. (1996). Pyruvate Metabolism in Saccharomyces cerevisiae. Yeast, 
Purahong, W., Wubet, T., Krüger, D. \& Buscot, F. (2018). Molecular evidence strongly supports deadwood-inhabiting fungi exhibiting unexpected tree species preferences in temperate forests, 289-295.

R Core Team. (2018). R: a language and environment for statistical computing. R Found. Stat. Comput. R Studio Team. (2016). RStudio: integrated development environment for R.

Ragazzi, A., Moricca, S., Capretti, P., Dellavalle, I., Mancini, F. \& Turco, E. (2001). Endophytic fungi in Quercus cerris: isolation frequency in relation to phenological phase, tree health and the organ affected. Phytopathol. Mediterr., 40, 165-171.

Rajala, T., Peltoniemi, M., Hantula, J., Mäkipää, R. \& Pennanen, T. (2011). RNA reveals a succession of active fungi during the decay of Norway spruce logs. Fungal Ecol., 4, 437-448.

Rajala, T., Peltoniemi, M. \& Pennanen, T. (2012). Fungal community dynamics in relation to substrate quality of decaying Norway spruce ( Picea abies [L.] Karst.) logs in boreal forests “, 81, 494-505.

Rayner, A.D.M. \& Boddy, L. (1988). Fungal decomposition of wood: its biology and ecology. Wiley.

Renvall, P. (1995). Community structure and dynamics of wood-rotting Basidiomycetes on decomposing conifer trunks in northern Finland. Karstenia, 35, 1-51.

Riley, R., Salamov, A.A., Brown, W., Nagy, L.G., Floudas, D., Held, B.W., et al. (2014). Extensive sampling of basidiomycete genomes demonstrates inadequacy of the white-rot / brown-rot paradigm for wood decay fungi, 111.

Rodriguez, R.J., Jr, J.F.W., Arnold, A.E. \& Redman, R.S. (2009). Fungal endophytes: diversity and functional roles. New Phytol., 182, 314-330.

Rondeux, J. \& Sanchez, C. (2010). Review of indicators and field methods for monitoring biodiversity within national forest inventories. Core variable: Deadwood. Environ. Monit. Assess., 164, 617-630.

Ruiz-matute, A.I. (2011). Derivatization of carbohydrates for GC and GC- MS analyses. Jounral Chromatogr. B, 879, 1226-1240.

Runnel, K., Tamm, H. \& Lõhmusa, A. (2015). Surveying wood-inhabiting fungi: most molecularly detected polypore species form fruit-bodies within short distances. Fungal Ecol., 18, 93-99.

Sarkanen, K. \& Ludwig, C. (1971). Lignins: occurrence, formation, structure and ractions. John Wiley \& Sons, New York.

Schoch, C.L., Seifert, K.A., Huhndorf, S., Robert, V., Spouge, J.L., Levesque, C.A., et al. (2012). Nuclear 
ribosomal internal spacer (ITS) region as a universal DNA barcode marker for Fungi. PNAS, 109, 6241-6242.

Schultz, T.P. \& Nicholas, D.D. (2000). Lignin influence on angiosperm sapwood susceptibility to whiterot fungal colonization: a hypothesis. In: Lignin: historical, biological and materials perspectives (eds. Glasser, W., Northey, R. \& Schultz, T.). American Chemical Society, ACS Symposium Series, pp. 205-213.

Schwarze, F. (2007). Wood decay under the microscope. Fungal Biol. Rev., 21, 133-170.

Schwarze, F., Engles, J. \& C, M. (2000). Fungal strategies of wood decay in trees. Springer, Berlin.

Seibold, S., Brandl, R., Buse, J., Hothorn, T., Schmidl, J., Thorn, S., et al. (2015). Association of extinction risk of saproxylic beetles with ecological degradation of forests in Europe. Conserv. Biol., 29, 382390.

Shao, S., Wen, G. \& Jin, Z. (2008). Changes in chemical characteristics of bamboo (Phyllostachys pubescens) components during steam explosion. Wood Sci. Technol., 42, 439-451.

Sinsabaugh, R. (1994). Enzymic analysis of microbial pattern and process. Biol. Fertil. Soils, 17, 69-74.

Sinsabaugh, R.L., Antibus, R.K., Linkins, A.E., McClaugherty, C.A., Rayburn, L., Repert, D., et al. (1993). Wood decomposition: nitrogen and phosphorous dynamics in relation to extracelklular enzyme activity. Ecology, 74, 1586-1593.

Smith, T.W. \& Lundholm, J.T. (2010). Variation partitioning as a tool to distinguish between niche and neutral processes. Ecography (Cop.)., 33, 648-655.

Šnajdr, J., Dobiášová, P., Větrovský, T., Valášková, V., Alawi, A. \& Boddy, L. (2011). Saprotrophic basidiomycete mycelia and their interspecific interactions affect the spatial distribution of extracellular enzymes in soil. FEMS Microbiol. Ecol., 78, 80-90.

Song, S.H. \& Vieille, C. (2009). Recent advances in the biological production of mannitol. Appl. Microbiol. Biotechnol., 84, 55-62.

Song, Z., Kennedy, P.G., Liew, F.J. \& Schilling, J.S. (2016). Fungal endophytes as priority colonizers initiating wood decomposition, 1-12.

Stein, S., Mirokhin, Y., Tchekhovski, D. \& Mallard, G. (2011). The NIST Mass Spectral Search Program for the NIST/EPA/NIH mass spectral library.

Stockland, J., Siitonen, J. \& Jonsson, B. (2012). Biodiversity in dead wood. Cambridge University Press, Cambridge, UK.

Straatsma, G., Ayer, F. \& Egli, S. (2001). Species richness, abundance, and phenology of fungal fruit 
bodies over 21 years in a Swiss forest plot. Mycol. Res., 105, 515-523.

Svensson, M., Dahlberg, A., Ranius, T. \& Thor, G. (2014). Dead branches on living trees constitute a large part of the dead wood in managed boreal forests, but are not important for wood-dependent lichens. J. Veg. Sci., 25, 819-828.

Tan, J., Pu, Z., Ryberg, W.A. \& Jiang, L. (2012). Species phylogenetic relatedness, priority effects, and ecosystem functioning. Ecology, 93, 1164-1172.

Taylor, D.L., Walters, W.A., Lennon, N.J., Bochicchio, J., Krohn, A., Caporaso, J.G., et al. (2016). Accurate estimation offungal diversity and abundance through improved lineage-specific primers optimized for Illumina amplicon sequencing, 82, 7217-7226.

Thurston, C. (1994). The structure and function of fungal laccases. Microbiology, 140, 19-26.

Toledo, S., Hiscox, J., Savoury, M. \& L., B. (2016). Multispecies interactions between wood decay basidiomycetes. J. Fungal Res.

Toljander, Y.K., Lindahl, B.D., Holmer, L. \& Högberg, N.O.S. (2006). Environmental fluctuations facilitate species co-existence and increase decomposition in communities of wood decay fungi. Oecologia, 148, 625-631.

Tordoff, G.M., Boddy, L. \& Jones, T.H. (2006). Grazing by Folsomia candida (Collembola) differentially affects mycelial morphology of the cord-forming basidiomycetes Hypholoma fasciculare, Phanerochaete velutina and Resinicium bicolor. Mycol. Res., 110, 335-345.

Tretter, L., Patocs, A. \& Chinopoulos, C. (2016). Succinate, an intermediate in metabolism, signal transduction, ROS, hypoxia, and tumorigenesis. Biochim. Biophys. Acta - Bioenerg., 1857, 1086-1101.

Ujor, V.C., Peiris, D.G., Monti, M., Kang, A.S., Clements, M.O. \& Hedger, J.N. (2012). Quantitative proteomic analysis of the response of the wood-rot fungus, Schizophyllum commune, to the biocontrol fungus, Trichoderma viride. Lett. Appl. Microbiol., 54, 336-343.

Unterseher, M., Otto, P. \& Morawetz, W. (2005). Species richness and substrate specificity of lignicolous fungi in the canopy of a temperate, mixed deciduous forest. Mycol. Prog., 4, 117-132.

Unterseher, M. \& Tal, O. (2006). Influence of small scale conditions on the diversity of wood decay fungi in a temperate, mixed deciduous forest canopy. Mycol. Res., 110, 169-178.

Valášková, V., de Boer, W., Klein Gunnewiek, P., Pospísek, M. \& Baldrian, P. (2009). Phylogenetic composition and properties of bacteria coexisting with the fungus Hypholoma fasciculare in decaying wood. ISME J., 3, 1218-1221.

Valmaseda, M., Almendros, G. \& Martínez, A.T. (1990). Substrate-dependent degradation patterns in the Page | 132 
decay of wheat straw and beech wood by ligninolytic fungi. Appl. Microbiol. Biotechnol., 33, 481-484.

Vanette, R. \& Fukami, T. (2014). Historical contingency in species interactions : towards niche-based predictions. Ecol. Lett., 17, 115-124.

Varnaitè, R. \& Raudonienè, V. (2008). Destruction of hemicellulose in rye straw by micromycetes. Ekologija, 54, 169-172.

Vellend, M. (2010). Conceptual synthesis in community ecology. Q. Rev. Biol., 85, 183-206.

Viana, D.S., Cid, B., Figuerola, J. \& Santamaría, L. (2016). Disentangling the roles of diversity resistance and priority effects in community assembly. Oecologia, 182, 865-875.

Wadekar, R. V, North, M.J. \& Watkinson, S.C. (2018). Proteolytic activities in two wood-decaying basidiomycete fungi, Serpula lacrymans and Coriolus versicolor. Microbiology, 141, 1575-1583.

Van der Wal, A., Gunnewiek, P.J.A.K., Cornelissen, J.H.C., Crowther, T.W. \& Boer, W. De. (2016). Patterns of natural fungal community assembly during initial decay of coniferous and broadleaf tree logs. Ecosphere, 7.

Wang, Y., Xiao, J., Suzek, T., Zhang, J., Wang, J. \& Bryant, S. (2009). PubChem: a public information system for analyzing bioactivities of small molecules. Nucleic Acids Res., 37, W623-W633.

Weedon, J.T., Cornwell, W.K., Cornelissen, J.H.C., Zanne, A.E., Wirth, C. \& Coomes, D.A. (2009). Global meta-analysis of wood decomposition rates: A role for trait variation among tree species? Ecol. Lett., $12,45-56$.

Weslien, J., Djupstrom, L.B., Schroeder, M. \& Widenfalk, O. (2011). Long-term priority effects among insects and fungi colonizing decaying wood. J. Anim. Ecol., 80, 1155-1162.

White, N.A. \& Boddy, L. (1992). Extracellular enzyme localization during interspecific fungal interactions. FEMS Microbiol. Lett., 98, 75-79.

White, T., Bruns, T., Lee, S. \& Taylor, J. (1990). Amplification and direct sequencing of fungal ribosomal RNA for phylogenetics. In: PCR protocols: a guide to methods and applications (eds. Innis, M., Gelfand, D., JJ, S. \& White, T.). Academic Press, San Diego, pp. 315-321.

White, W.H., Gunyuzlu, P.L. \& Toyn, J.H. (2001). Saccharomyces cerevisiae is capable of de novo pantothenic acid biosynthesis involving a novel pathway of $\beta$-alanine production from spermine. J. Biol. Chem., 276, 10794-10800.

Wiemken, V. (2007). Trehalose synthesis in ectomycorrhizas - a driving force of carbon gain for fungi? New Phytol., 174, 228-230.

Xie, J., Qi, J., Hse, C.Y. \& Shupe, T.F. (2014). Effect of lignin derivatives in the bio-polyols from 
microwave liquefied bamboo on the properties of polyurethane foams. BioResources, 9, 578-588.

Xu, X.Y., Shen, X.T., Yuan, X.J., Zhou, Y.M., Fan, H., Zhu, L.P., et al. (2018). Metabolomics investigation of an association of induced features and corresponding fungus during the Co-culture of trametes versicolor and Ganoderma applanatum. Front. Microbiol., 8, 1-14.

Yaoi, K. \& Mitsuishi, Y. (2002). Purification, characterization, cloning, and expression of a novel xyloglucan-specific glycosidase, oligoxyloglucan reducing end-specific cellobiohydrolase. J. Biol. Chem., 277, 48276-48281.

Yim, G., Wang, H.H. \& Davies, J. (2007). Antibiotics as signalling molecules. Philos. Trans. R. Soc. B Biol. Sci., 362, 1195-1200.

Zhang, B., Zhang, J., Liu, Y., Guo, Y., Shi, P. \& Wei, G. (2018). Biogeography and ecological processes affecting root-associated bacterial communities in soybean fields across China. Sci. Total Environ., $627,20-27$.

Zhang, J. \& Schilling, J.S. (2017). Role of carbon source in the shift from oxidative to hydrolytic wood decomposition by Postia placenta. Fungal Genet. Biol., 106, 1-8.

Zhong, Z., Li, N., Liu, L., He, B., Igarashi, Y. \& Luo, F. (2018). Label-free differentially proteomic analysis of interspeci fi c interaction between white-rot fungi highlights oxidative stress response and high metabolic activity. Fungal Biol.

Zhou, S., Zhang, J., Ma, F., Tang, C., Tang, Q. \& Zhang, X. (2018). Investigation of lignocellulolytic enzymes during different growth phases of Ganoderma lucidum strain G0119 using genomic , transcriptomic and secretomic analyses, 1-20. 


\section{Appendix I}

The following data files are available on request:

S1 Branch Community Maps

S2 Branch Community DNA Consensus Sequences

S3 Species List and Taxonomy / Functional Classification Data

S4 BEST Analysis Output

S5 Relative Abundances of Selected Species over Mass Loss and Decay Gradients at Various Taxon and Functional Levels

S6 Full Community Data Set and distLM Outputs

This file can be opened in PRIMER 7. A 7 day free trial is available from http://www.primer-e.com

S7 Branch Community SAR Correlations 


\section{Appendix II}

The following data files are available on request:

S8 Branch Communities Enzyme Assay Results

S9 Branch Communities GC-MS Chromatograms

S10 Branch Communities Pre-processed Metabolome Data

S11 Branch Communities Putatively Identified Metabolites

S12 Branch Full Metabolome PLS-DA Pairings Plot

S13 Branch Metabolome VIP analysis

S14 Branch Metabolome Volcano Plot Data

S15 Branch Metabolite Enzyme Activities distLM Output

S16 Branch Metabolite $\sim$ Branch Variables distLM Output 


\section{Appendix III}

The following data files are available on request:

S17 Woodblock Agilent GC-MS Chromatograms

S18 Chapter 5 - Putatively Identified Metabolites

S19 Chapter 5 - Metabolome of Priority Effects Experiment

S20 Chapter 5 - Priority effects Differential Metabolomics Analyses Output

S21 Chapter 5 - One-way ANOVA of Exidia glandulosa metabolome following along-vessel replacement interactions

S22 Chapter 5 - Two-way ANOVA results

S23 Chapter 6 - Metabolome of Oudemansiella mucida in Temperature Manipulation Experiment

S24 Chapter 6 - Metabolome of Exidia glandulosa in Temperature Manipulation Experiment

S25 Chapter 6 - Differential Metabolomics Analyses Output - O. mucida Temperature Exp.

S26 Chapter 6 - Differential Metabolomics Analyses Output - E. glandulosa Temperature Exp.

S27 Chapter 6 - Putatively Identified Metabolites in Temperature Manipulation Experiment 


\section{Appendix IV}

The following data files are available on request:

S28 MaxQuant Data Provided by Maynooth University (Chapters 5 \& 6)

S29 Key to Sample References in S29

S30 Protein Sequences Data provided by Maynooth University (Chapters 5 \& 6)

S31 Chapter 5 - Gene Ontology Classifications - Primary and Secondary Resource Capture

S32 Chapter 5 - Proteins Pathway Analysis

S33 Chapter 5 - Directed Acyclic Graphs

S34 Chapter 6 - Gene Ontology Classifications - Exidia glandulosa and Oudemansiella mucida

S35 Chapter 6 - Directed Acyclic Graphs

S36 Chapter 6 - PLS-DA and T-test Analyses of O. mucida proteome under temperature manipulation

S37 Chapter 6 - PLS-DA and T-test Analyses of E. glandulosa proteome under temperature manipulation

S38 Chapter 6 - Proteins Pathway Analysis 